\title{
Human and mouse meniscus progenitor cells and their role in meniscus tissue regeneration
}

\author{
Dissertation \\ for the award of the degree \\ "Doctor of Philosophy (Ph.D.)" \\ Division of Mathematics and Natural Sciences \\ of the Georg-August University, Goettingen
}

\author{
Submitted by \\ Hayat Muhammad \\ from Darazinda, F. R. D. I. Khan, Pakistan
}

Goettingen, 2014 


\section{Thesis Committee}

Prof. Dr. Nicolai Miosge

Head, Oral Biology and Tissue Regeneration Work Group

Department of Prosthodontics, Medical Faculty,

Georg August University, Robert Koch Str. 40,

37075, Goettingen, Germany

Prof. Dr. Sigrid Hoyer-Fender

Johann-Friedrich-Blumenbach institute for Zoology and Anthropology

Department of Developmental Biology, GZMB,

Georg August University, Justus-von-Liebig-Weg 11,

37077 Goettingen, Germany

Members of the Examination Board Reviewers (in alphabetical order)

Prof. Dr. Gehard Braus

Institute for Microbiology and Genetics,

Department of Molecular Microbiology and Genetics,

Georg August University, Grisebachstr. 8, 37077

Goettingen, Germany

Prof. Dr. Uwe Gross

Institute for Medical Microbiology, Medical Faculty

Georg August University, Kreuzbergring 57,

37075, Goettingen, Germany

Prof. Dr. Michael Kessel

Max Planck Institute for Biophysical Chemistry

Department of Developmental Biology

Am Fassberg 11, 37075 Goettingen, Germany

Prof. Dr. Ernst Wimmer

Johann-Friedrich-Blumenbach institute for Zoology and Anthropology,

Department of Developmental Biology, GZMB,

Georg August University, Justus-von-Liebig-Weg 11,

37077 Goettingen, Germany

Date of the oral examination: May , 2014 


\section{Contents}

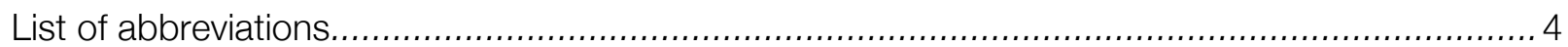

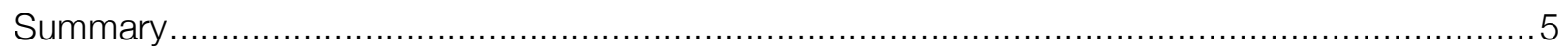

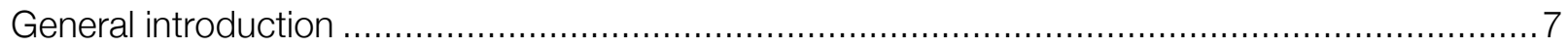

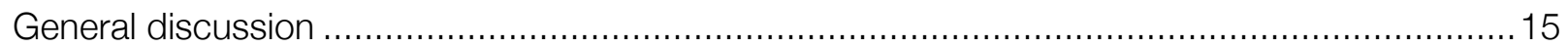

Chapter 1: Current concepts in stem cells therapy for articular cartilage repair .......................19

Chapter 2: Primary cilium as dual sensor of mechanochemical signals in chondrocytes............28

Chapter 3: A discoidin domain receptor 1 knock-out mouse as novel model for osteoarthritis

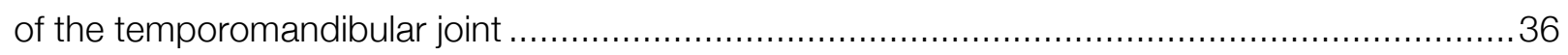

Chapter 4: Human migratory meniscus progenitor cells are controlled via the TGF $\beta$ pathway ....57

Chapter 5: Identification and characterization of mouse meniscus progenitor cells in vitro and

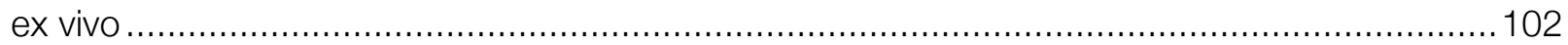

Chapter 6: Use of chondrogenic progenitor cells in osteoarthritis ......................................123

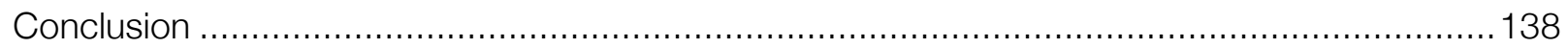

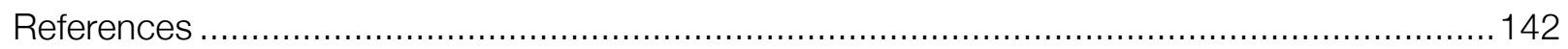

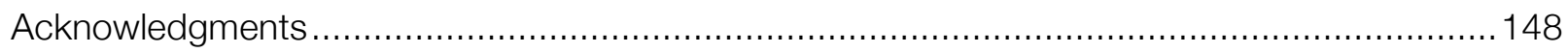

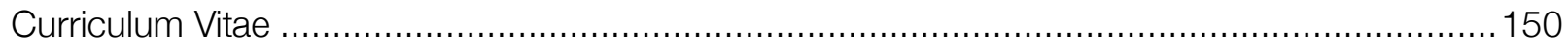


List of abbreviations

BMP

CPCs

CD

DDR-1

ECM

GDF-5

$\mathrm{KO}$

MSCs

MPCs

MMPCs

MMP

OA

RTK

Runx2

Sox9

SMAD

TGF $\beta 3$

TMJ

TMD

TIMP
Bone morphogenetic protein

Chondrogenic progenitor cells

Cluster of differentiation

Dicoidin domain receptor

Extracellular matrix

Growth differentiation factor 5

Knockout

Mesenchymal stem cells

Human meniscus progenitor cells

Mouse meniscus progenitor cells

Matrix metalloproteinase

Osteoarthritis

Receptor tyrosine kinases

Runt-related transcription factor 2

SRY (sex determining region Y)-box 9

small 'mothers against' decapentaplegic

Transforming growth factor beta 3

Temporomandibular joint

Temporomandibular disorders

Tissue inhibitor of metalloproteinase 


\section{Summary}

Osteoarthritis $(\mathrm{OA})$ is a degenerative disease of hyaline cartilage. The cartilage is an avascular tissue, once damaged it is difficult to repair. Articular cartilage is responsible for the smooth, frictionless, and painless moment of the knee joint. Severe loss or complete destruction of the articular cartilage results in highly frictional and painful moment of the knee joint, as is often the case in the late stages of OA. Total joint replacement remains the ultimate solution. However, there are many other options emerging to repair or regenerate the cartilage using various types of stem cells, but they might lead to some serious consequences. For example, transplantation of embryonic stem cells may result in teratoma formation. Applications of induced pluripotent stem cells are associated with possible viral integration. Alternatively, the concept of progenitor or repair cells in situ arises, and, for example, migratory chondrogenic progenitor cells (CPCs) were found in the later stages of human osteoarthritic cartilage in vivo. They have a tremendous ability for cartilage repair with no serious risk factors known so far. However, CPCs still face some challenges, for example, how to provoke them in situ to repair the tissue in a physiological manner. Secondly, CPCs have a limited life span at least in vitro (chapter 1). Furthermore, so far, there is no single optimized method available to achieve a complete chondrogenic differentiation of stem cells. More recently, a tiny organelle of the cells, known as primary cilium might be found to be instrumental for stem cell differentiation. This 'physical projection' of the cell acts like an antenna, and is considered as dual sensor of mechanochemical signals. This sensor has also been found on CPCs and their numbers have been shown to be reduced on chondrocytes derived from the temporomandibular joint (TMJ) of the discoidin domain receptor 1 knockout (DDR-1 KO) mouse (chapter 2, 3).

OA is not only restricted to large joints but also affects small joints like the TMJ. It is well known that chondrocytes in the articular cartilage do not make direct cell-to-cell contacts, instead they rely on cell-matrix-interactions via cell receptors, for example, integrins or DDRs for their communication. DDR-1 KO mice exhibit typical symptoms of $\mathrm{OA}$ of the TMJ cartilage. The chondrocytes derived from the TMJ cartilage of DDR-1 KO mice maintained their osteoarthritic characteristics such as a high expression of runx2 and collagen type I as typical osteogenic signature, and a low expression of sox9, collagen type II and aggrecan known to be relevant for the chondrogenic differentiation. 
The osteoarthritic characteristic could be reversed to a more normal chondrocyte type via the knockdown of runx2 or exposure of these cells to a three-dimensional environment in the presence of extracellular matrix (ECM) components such as laminins and nidogen (chapter 3). Their reversal towards the chondrogenic phenotype would also be of great importance in the pathogenesis of meniscus degeneration. Meniscus most often is the starting point for the development of OA of the knee joint. The meniscus is a fibrocartilaginous tissue, which acts as shock absorber. Furthermore, meniscus damaging makes the whole scenario of OA pathogenesis worse, by enhancing the cartilage degradation. The inner part of the meniscus is avascular in nature and therefore, once damaged it has a very limited self-repair capacity. However, there are other options available like the partial removal of the meniscus, which results in a short-term relief, but cannot prevent the long-term consequences, which ultimately lead to the development of $\mathrm{OA}$. The inner part of the human meniscus harbors unique meniscus progenitor cells (MPCs) and can be directed towards meniscus regeneration via the TGF $\beta$ signaling pathway (chapter 4). Additionally, mouse meniscus progenitor cells (MMPCs) were investigated in healthy (wild type) meniscus tissue. These cells were traced ex vivo using immuno-histochemical techniques. They also maintained their stem cell characteristics in vitro as well, as shown via their stem cell marker expression and their mulitipotency (Chapter 5).

Many critical consequences are associated with the application of various stem cells for cartilage regeneration as a therapy. Here, I concentrated on tissue specific cells, progenitor cells in situ, already present in diseased cartilage. These cells are well determined towards chondrogenic differentiation and might only need minor manipulations to fulfill their final determination to produce native-like hyaline cartilage (chapter 6). 
General introduction

Osteoarthritis and cartilage tissue

$\mathrm{OA}$ is a chronic and degenerative joint disease. $\mathrm{OA}$ is a multifactorial disease, many important factors contribute to OA of the knee joint, such as age, obesity, trauma, joint anatomy, genetics or extensive use of the body joints in a daily life style of an individual (Blagojevic et al, 2010). Cartilage is responsible for the smooth, frictionless and painless moment of the knee joint. A healthy articular cartilage is a distinctively designed connective tissue, which covers the articular ends of the long bones. Articular cartilage is a highly specialized, avascular, alymphatic and aneural tissue. Biomechanically, it is a flexible tissue. Its integrity and functions solely depends on its ECM. Generally, chondrogenesis is initiated by mesenchymal stem cells (MSCs) condensation and chondroprogenitor cells differentiation into chondrocytes. Articular cartilage is synthesized by resting chondrocytes. While for bone formation, chondrocytes undergo terminal hypertrophic differentiation and apoptosis. Finally, the hypertrophic cartilage is replaced by cells of the osteoblastic lineage, a process called endchondral ossification (Goldring, 2012). However, in hyaline cartilage, only a single cell type, the chondrocyte, is responsible for the synthesis of the ECM (Kuettner, 1992). The ECM of articular cartilage is rich in two major components, the collagen molecules and proteoglycans. The most abundant collagen found in articular cartilage is collagen type II. However, normal healthy cartilage also possesses types, III, VI, IX, X, XI, XII and XIV collagens (Mayne \& Brewton, 1993). The second major components are proteoglycans, which are a heterogeneous group of proteins, comprising a central core protein substituted with one or more glycosaminoglycans side chains. For example, the aggrecan, as a large proteoglycans, is responsible for cartilage maintenance as are the other small proteoglycan such as decorin, biglycan and fibromodulin (Knudson \& Knudson, 2001; Poole, 1999).

DDR-1 Knockout results in OA of the TMJ-cartilage

The pathogenesis of OA is always linked to a disordered ECM-cell relationship. In healthy articular cartilage the chondrocytes are trapped in a collagen scaffold tightly packed by glycoproteins and proteoglycans, which serve as bridges to stabilize the collagen network (Kruegel et al, 2008). Chondrocytes in articular cartilage are apart from each 
other. However, they communicate via cell-matrix interactions (Kuettner, 1992), for example, via discoidin domain (DDRs) and integrin receptors (Goldring \& Otero, 2011; Loeser, 2000). DDRs are receptor tyrosine kinases (RTKs) and are normally expressed in human and mouse tissues. The RTKs are activated extracellulary by binding to collagen molecules (Vogel, 1999). Further, the activated RTKs switches on downstream signaling processes via SHC (src homology domain-containing) (Vogel et al, 1997) or FRS2 (fibroblast growth factor receptor substrate 2) (Foehr et al, 2000) and alter the expression level of genes related to ECM production (Poole, 1999), which are required for the homeostasis of healthy articular cartilage. In this study, the DDR-1 KO mouse is introduced to exhibit a high incidence of $\mathrm{OA}$ in the TMJ. For example, it shows degenerative signs such as, surface fissures and high expression of collagen type I. It is anticipated that temporomanidbular disorders (TMD) affect both the young and aged (18 to 45 age). This is around $10-40 \%$ of the population and $10 \%$ among them result in OA of the TMJ. Anyhow, untreated TMDs ultimately result in OA of the TMJ (Wadhwa et al, 2005). The chondrocytes derived from TMJ cartilage can be directed towards normal chondrocytes via a runx2 knockdown, which results in an up-regulation of both collagen type II, sox9 and down-regualtion of collagen type I. The OA characteristics of the DDR-1 $\mathrm{KO}$ cell, for example, high collagen type I and high runx2 expression, were significantly reduced in three-dimensional cells cultures in the presence of laminin, BMP-6 and nidogen-2, respectively. Here, we came up with new chondrogenic differentiation factors namely basement membrane associated proteins (laminin and nidogen-2) in combination with bone morphogenic protein-6.

Chondrocytic primary cilium in osteoarthritis

Articular cartilages are always exposed to mechanical forces especially the knee joint and possess very limited self-repair capability. Recently, a cell's organelle is gaining a high interest, which is considered as mechnochemical sensor of the cell, known as primary cilium (Muhammad et al, 2013; Poole et al, 1997; Poole et al, 2001). It has been proved by electron microscopic studies that chondrocytes have a non-motile primary cilium. Furthermore, its physical orientation has been shown in the pericellular matrix of the chondrocyte and its binding via a receptor with collagen type II and collagen type IV (Jensen et al, 2004; McGlashan et al, 2006; Wilsman et al, 1980). Physical or genetic mutation of the chondroblastic and chondrocytic primary cilium results in a malformation 
of ECM, which causes skeletal and growth plate abnormalities (Gouttenoire et al, 2007; Haycraft et al, 2007; Ruiz-Perez et al, 2007). It has been examined that the primary cilium is present on chondrocytes during all stages of OA. The number of primary cilia were found to be increased in OA. However, this was studied in a bovine model (McGlashan et al, 2008; Wilsman, 1978). Here in this study, a slight reduction of primary cilia was observed on chondrocytes derived from DDR-1 KO mice (Schminke et al, 2014). The organelle, the primary cilium, become visible also on CPCs using fluorochemical techniques (Muhammad et al, 2012).

\section{Human Chondrogenic progenitor cells}

In the late stage of human OA the articular cartilage is distorted, and for example, the repair tissue is found to be mainly composed of collagen type I produced by repair cells. Recently, Prof. Miosge's lab isolated a subpopulation of these cells, termed chongrogenic progenitor cells (CPCs). The CPCs exhibits stem cells characteristics. They are found to be positive for stem cell markers, for example, CD105, CD90, CD44, CD29 and CD73. The CPCs have been shown to be easily driven towards chondrogenesis via a runx2 knockdown or their exposure to chondorgenic media in alginate culture (Koelling et al, 2009; Muhammad et al, 2013). Both runx2 and sox9 are master regulators responsible for bone development and articular cartilage development, respectively. Higher expression of sox9 encourages chondrogenesis. While high expression of runx2 inhibits chondrogenesis (Banerjee et al, 1997; Lefebvre \& Smits, 2005; Wuelling \& Vortkamp, 2011).

Human meniscus progenitor cells

The articular cartilage in the knee joint is protected by menisci. Menisci are fibrocartilagenous tissues, which give structural support to the knee joint. The meniscus does not allow direct exposure of the femuro-tibial articular cartilage surfaces during the mechanical movement of the knee joint and is considered as safeguard, which covers the surfaces of articular cartilage. It is located at the interface of the femoral condyles and the tibial plateau. The meniscus is a semi-lunar structure, the central region is known as 'body', the larger posterior and anterior portions are called posterior and anterior horns, respectively. Approximately, the average size of the meniscus is $3.5 \mathrm{~cm}$ in length (Kohn \& 
Moreno, 1995). Fibrochondrocytes are the main cells of the meniscus, responsible for fibrocartilagenious matrix secretion. Usually, oval fibrochondrocytes reside in lacunae (Hellio Le Graverand et al, 2001a). Meniscus tissue is more complex than articular cartilage in terms of its cellular and ECM composition. The inner part of the human meniscus is avascular, termed white zone. While, the outer part of the meniscus is vascularized, termed red zone. The superficial zone is under the pressure of the total body weight, which alters the cell into a flattened phenotype. The cells with a flattened phenotype are secreting collagen type I. Histologically, when observing zones away from the pressure zone, the inner zone cells are round in shape. They are like proper chondrocytes, producing more collagen type II and less collagen type I (Pauli et al, 2011; Zhang et al, 2011).

It has been known that a healthy human meniscus tissue is mainly composed of $72 \%$ water, 22\% collagen, $0.8 \%$ glycosaminoglycans (GAGs) and 0.12\% DNA. Adult menisci possess $78 \%$ collagen, $8 \%$ non-collagenous protein and $1 \%$ hexose amine. However, these observations were calculated on a dry weight basis (Ingman et al, 1974). The collagen type I is the major component of the meniscus ECM, which contributes $90 \%$ of the total collagen. Meniscus tissue also possesses type II, III and V collagen (Eyre \& Wu, 1983; McDevitt \& Webber, 1990). Interestingly, collagen type I has been observed in the outer region of the meniscus. Contrary, collagen type II is located only in the central part, which shows cartilage-like properties (Chevrier et al, 2009). The ECM is responsible for the mechanical stability and integrity to the meniscus tissue. Malformations of collagen and non-collagen proteins have been found in the degenerated meniscus tissue. This means, menisci with a poor ECM composition are more susceptible to damage (Herwig et al, 1984; Ingman et al, 1974).

In order to understand the developmental biology of meniscus, for example, how the menisci are derived embryonically one should take into account the overall developmental phenomena of normal synovial joint formation. The developmental process begins with the condensation of the mesenchymal blastema and this differentiates into a cartilaginous model, which serves as template for long bone formation. A thin layer of MSCs, known as interzone, separates the adjacent skeletal parts of the joint. Later, the interzone is differentiated into three layers. Both of the outer layers are chondrogenic and responsible to develop into the cartilage anlage. The middle 
layer is mainly involved in the formation of intra-articular tissues such as meniscus, ligament and the synovium (Ito \& Kida, 2000). It has been reported that mechanical stimulation assists the maintenance of newly developed meniscus during embryogenesis. Mechanical deficiency, for example, non-functional muscle contraction alters meniscus condensation (Mikic et al, 2000).

The meniscus organogenesis in respect to the expression of matrix related genes can be divided into four steps. Initially, MSCs condensation occurs. Secondly, the differentiation of meniscal fibrochondrocytes takes place in the newly formed meniscus tissue. Thirdly, the protein synthesis machinery of the cells starts to synthesize meniscal ECM. Finally, mature ECM is excreted, which traps the meniscal cells (Pavlova et al., 2001).

The above stages of meniscus development have been investigated in detail. For example, during the first stage of condensation, MSCs start to express BMP-4 and GDF5, which differentiates the cells more towards the meniscal fibrochondroblast. Interestingly, the chondrocyte-like cells are formed accidently during the phase of low expression of BMP-4 and GDF-5. Further, first the cells synthesize collagen type I, collagen type III and aggrecan. However, the collagen type II synthesis occurs in the late stages of meniscus organogenesis (Pavlova et al., 2001)

The inner one-third regions of the menisci in early development are mostly vascularized. This could be identified in the middle of the human gestation period, usually, after 150 days (Petersen \& Tillmann, 1995). The meniscus is totally vascularized at birth. Gradually, the meniscus becomes avascularized. Particularly, avascularization begins at the second year of age and it starts in the central part of the meniscus (Petersen \& Tillmann, 1998). It has been shown that damage of the vascularized zone heals as easily as the other vascular tissues of the body (Arnoczky \& Warren, 1983; Heatley, 1980; King, 1990). In contrast, a tear located in the avascularized zone, restores poorly (Henning et al, 1990; King, 1990). To enhance the repair of the central, avascular zone, it has been connected to the vascular region by surgical incision (Arnoczky \& Warren, 1983; Zhang et al, 1995). Although there have been many efforts to heal the inner zone of the meniscus, clinically it has remained a great challenge (Henning et al, 1990). It has been shown that the avascular part of the meniscus has no intrinsic self-repair capability. Already partial 
removal of a meniscus has deleterious effects and eventually results in OA (Arnoczky \& Warren, 1983; Hasan et al, 2014; Walker \& Erkman, 1975).

Degenerated or injured menisci are usually found to be calcified and it is challenging to stop the calcification process (Noble \& Hamblen, 1975). Stem cell biologists came up with cell therapeutical approaches, such as, the application of MSCs. In principle, MSCs are considered a good choice, but it is still difficult to differentiate these cells towards the desired meniscus cell type and also difficult to maintain its phenotype. Differentiation often results in the production of an inappropriate ECM and mostly favors hypertrophy, as terminal differentiation (Mauck et al, 2006; Pelttari et al, 2006). However, efforts have been made by researchers to overcome the problems of hypertrophy and to control the undesired ECM synthesis. For example, Bian et al. came up with the concept of coculturing MSCs together with chondrocytes in hydrogels (Bian et al, 2011). It has also been shown that hypertrophy can be avoided when MSCs are co-cultured with meniscus derived cells (Cui et al, 2012). From the tissue engineering point of view, MSCs plus fibrin glue were found to be a good combination for healing of meniscus tissue (Ferris et al, 2012). Surprisingly, MSCs derived from other adult tissues have been tested for meniscus regeneration, such as, cells of the synovial membrane. The synovial membrane derived MSCs are highly acknowledged by scientists for meniscus tissue regeneration, as they acquire a high chondrogenic potential (Katagiri et al, 2013; Sakaguchi et al, 2005). Although MSCs have revealed some satisfactory results for meniscus regeneration in a temporarily way, they are not promising in the long run.

The main focus of this study is related to osteoarthritic degenerative lesions of the meniscus, which should be distinguished from traumatic injury of the meniscus. The term 'degenerative lesion' reflects damaging of the meniscus without an injury. In the present literature, the association of $\mathrm{OA}$ and degeneration of the meniscus is pathomechanistically unclear and still debatable. However, it is known that the degeneration of the meniscus tissue is occurring during the course of OA.

In this study, it has been found that mostly the superficial zone of the meniscus is highly degenerated in the late stages of human OA. Interestingly, the damaged meniscus harbors MPCs, they are present in the inner zone. MPCs possess stem cell-like characteristics, for example, they are positive for well known stem cells markers (CD44, 
CD105, CD106, Stro-1, CD90 and CD29). They also exhibit multipotency and can be differentiated to three mesenchymal lineages (chondrocytes, adipocytes and cells of the osteoblastic lineage). In short, all OA related symptoms were observed in highly diseased meniscus, for example, degeneration of the superficial zone, high expression of collagen type I, runx2 and extremely low level of collagen type II and sox9. Further, results showed a suppression of the TGF $\beta$ pathway. This low expression of TGF $\beta 3$, smad2, sox9 as well as an up-regualtion of collagen type I and runx2 were observed in diseased specimens. This lead to experiments to drive MPCs towards chondrogenesis by different cell biological methods, such as, the induction with TGF $\beta 3$. TGF $\beta 3$ stimulates p-smad2, which in turn suppressed runx2 expression and enhances sox9 expression. Smad2 overexpression results in down-regulation of runx2 and up-regualtion of sox9. Further, the reverse effect of runx2 was tested via a runx2 knockdown. Runx2 silencing resulted in the up-regulation of smad2. Importantly, p-smad2 becomes detectable only after the runx2 knockdown. On the other hand, the cell induction experiment with BMP-2 results in a low expression of smad2 and sox9.

Mouse meniscus progenitor cells

In this study, also MMPCs were investigated. MMPCs possess stem cells like characteristics. For example, these cells were found to be positive for stem cell markers, for example, CD29, CD73, CD90 and CD44. They also showed multipotency and could be differentiated towards the cells of the osteoblastic lineage and adipocytes. MMPCs have been derived from the inner, avascular part of the meniscus and showed negativity for hematopoietic stem cells markers, such as, CD34 and CD117. Previously, It has been reported that the outer, vascular part of the human meniscus is rich in stem cells which are also positive for hematopoietic stem cell markers (Osawa et al, 2013). Therefore, MMPCs are different from the previously published cells.

In addition, it was noticed that mouse meniscus has a different histological pattern compared to human. For example, the main ECM protein, collagen type I, is not uniformly distributed as in human and is found only in the outer, avascular part of the mouse meniscus. The high weight bearing superficial zone is absent in mouse meniscus. 
Conclusion

Overall, the main theme of the project was to introduce novel progenitor cells. Particularly, for the regeneration of an avascular tissue such as cartilage or the inner part of the meniscus, which have limited intrinsic repair capabilities. Here, I introduced for the first time MPCs and MMPCs. They have stem cell-like characteristics and could be differentiated to three mesenchymal linages (cells of the osteoblastic lineage, adipocytes and chondrocytes). MPCs can be directed towards meniscus regeneration via the canonical TGF $\beta$ pathway. 
General discussion

\section{DDR-1 Knockout mice as an OA model}

The DDR-1 depletion results in several severe consequences, which encourage the pathogenesis of OA, for example, high expression of DDR-2, MMP-13, collagen type I and runx2. In order to elucidate the pathomechanism of DDR-1 KO mice, numerous approaches were applied to chondrocytes derived from DDR-1 KO TMJs. The DDR-1 $\mathrm{KO}$ chondrocytes revealed a high expression of runx2 and collagen type I, as well as low levels of sox9 and collagen type II. It has been reported that this catabolic shift in chondrocytes encourages the progression of OA development (Kamekura et al, 2006). Interestingly, the DDR-1 KO results in the high expression of the DDR-2 isoform, which in turn enhanced the expression of MMP-13. MMP-13 is mainly responsible for articular cartilage destruction. The PCR array (mouse signaling pathways) of DDR-1 KO chondrocytes showed a modulation of the $I H H$ (Indian hedgehog) signaling pathway, which is associated with OA of mouse and human (Lin et al, 2009).

The most exciting feature of the DDR-1 KO mice is that they developed OA of the TMJ only and by an early age of 9 weeks. OA related symptoms were not observed in the knee joint of DDR-1 KO mice. These findings can be addressed by a basic concept in developmental biology. Both joint's (knee and TMJ) cartilages are developed from different embryonic regions. TMJ cartilage is also considered a secondary cartilage (Shen \& Darendeliler, 2005; Symons, 1965). Additionally, the articular cartilage surfaces of the larger joints possess collagen type II. Contrary, this feature is absent in the superficial zone of the TMJ cartilage. However, the functional importance of this difference is unknown (Wadhwa et al, 2005). DDR-1 aberration induces MMP-13 expression, which in turn decreases collagen type II and initiates the development of OA in the TMJ. The regenerative activities were examined in a degenerated cartilage of the TMJ and found to be similar to human OA of the knee joint. Furthermore, the TMJ chondrocytes are also regulated by runx2 and sox9, as observed in CPCs (Koelling et al, 2009; Koelling \& Miosge, 2010). TMJ chondrocytes were rescued from the OA phenotype via knockdown of runx2, which in turn up-regulates sox9 and collagen type II, while collagen type I was highly suppressed. Likewise effects were observed for aggrecan (Bi et al, 1999) and COMP (Tallheden et al, 2004). Interestingly, DDR-2 was found to be highly active in the 
absence of DDR-1, which triggers signaling responses to the cytoplasm via binding to collagen type II molecules. In OA cartilage its binding affinity to collagen type II increases, which keeps the expression of MMP-13 switched on permanently (Xu et al, 2007). TMJ chondrocytes derived from DDR-1 KO mice were examined for primary cilia and showed a slight reduction of the primary cilia numbers. This suggests that the primary cilium is involved in the pathogenesis of OA in DDR-1-deficient chondrocytes.

The main aim of the study was to introduce DDR- $1 \mathrm{KO}$ mouse as a novel model for OA of the TMJ. Comparatively, the DDR-1 null mouse model exhibited a higher incidence of OA symptoms than previously published models, such as, the ICR mouse (Silbermann \& Livne, 1979), the Del 1 mouse (Rintala et al, 1997) and Cho mouse (Xu et al, 2003). The DDR-1 KO mouse will assist to explore more about the pathogenesis of OA of the TMJ in the future.

Human osteoarthritic meniscus harbors progenitor cells

All the typical symptoms related to OA have been observed also in the human meniscus, in the later stages of OA (Loeser et al, 2012), for example, tissue calcification, fatty degeneration and cells cluster formation (Hellio Le Graverand et al, 2001b). A simple histopathological grading system was applied and evaluated via transcriptomics and proteomics in order to extricate highly diseased meniscus from less diseased meniscus. Interestingly, an increased disease score also coincides with low immunohistochemical staining for TGF $\beta 3$ and smad2. At this point, the elucidation of the TGF $\beta / B M P$ pathway was considered in greater detail. BMP binds to its receptors and activates cytoplasmic mediators (smad1, smad5, smad8). One of the important downstream targets is runx2, which favors osteogenesis. Similarly, TGF $\beta$ bind to its receptors and stimulates smad2, smad3, and smad4. One of the important downstream targets is sox9, which encourage chondrogenesis. This dual effect of either promoting osteo- or chondrogenesis has been described by Massague (Massague, 2012). It has been shown that hyaline articular cartilage tissue harbors progenitor cells. A FACS analysis revealed that human MPCs are positive for well-known stem cells markers, such as, CD29, CD90, CD105, CD106 and stro-1. Interestingly, the explant culture-derived cells showed a higher expression of stem cells markers as compared to cells derived via collagenase digestions. This signifies that the MPCs are a subpopulation of these cells found in highly diseased human meniscus 
tissue. Furthermore, these cells were found to be multipotent and can be differentiated towards the three mesenchymal lineages (cells of osteoblastic, chondroblastic and adipoblastic lineages). After exposing the cells to osteogenic medium, they expressed osteogenic makers such as osteocalcin and alkaline phosphatase. Quite remarkably, in three dimensional cell cultures, they expressed collagen type II and suppressed runx2 under the influence of chondrogenic medium. Adipogenic differentiation was confirmed by oil red staining.

These cells also showed high migratory activity towards a gradient of platelet-derived growth factor (PDGF). Their migration and integration capability were examined by an ex vivo migration assay. MPCs showed a tremendous migratory and retention capability in diseased meniscus tissue. Taken together, the distinctive stem cell-related characteristics of these cells, such as, clonicity, multipotency and migratory potential, allowed to name the cells meniscus progenitor cells (MPCs). These cells are rather progenitors cells, because they show signs of senescence. MPCs derived from diseased meniscus revealed a low expression of smad2/smad3 and a high expression of smad7, a cytoplasmic inhibitor of the TGF $\beta$ pathway. In OA, it has been shown that depletion of the TGF receptor II results in boosting up runx2, MMP13 and ADAMS5 (Shen et al, 2013). The knockdown of runx2 elevated the total smad2/3, and more importantly, the psmad2. The MPCs induction with TGF $\beta 3$ also had a positive influence on cells in terms of their chondrogenesis, as shown by significantly reduced runx2 level. In turn, it upregulated the sox9 and p-smad2. In contrast, MPCs treated with BMP-2 totally reflected an adverse effect. It down-regulates sox 9 and smad2 expression, rather enhancing the osteogenic level.

In short, the altered level or shift from smad2/3 to smad1/5 seems to be important in the progression of $\mathrm{OA}$ in human meniscus. These findings suggest that specific mediators of the TGF $\beta$ /BMP pathway may serve as drug targets in regenerative medicine in the future, particularly for the late stages of OA of human meniscus. 
Mouse meniscus possesses progenitor cells

Additionally, in this study it has been examined that, the avascular region (inner part) of mouse meniscus harbors multipotent progenitor cells found to be positive for stem cell markers (CD44, CD73, CD29 and CD90). Stem cell marker positive cells were localized via immunohistochemistry, immunocytochemistry, PCR, FACS and Western blots. The MMPCs were found to be negative for hematopoietic stem cells markers (CD117 and CD34), as expected, as they derived from the avascular part.

More recently, Osawa et al. isolated cells from the vascular part of the meniscus via collagenase digestion and found them to be positive for hematopoietic stem cells marker, such as CD117 and CD34 (Osawa et al, 2013). It suggests that blood vessels or pericytes will be the possible source of their cells. Contrary, MMPCs are not only different from the Osawa et al. cells in terms of their source but also in terms of the methodological approach, which has been applied for cells isolation. Surprisingly, histological examination showed that structurally mouse meniscus is not completely identical to human meniscus. Mainly, a deviation has been observed in the superficial zone. A true superficial zone is absent in mouse meniscus and no flattened cells were seen as is in humans. Further, the structural divergence was confirmed by collagen type I staining, which intensively stained for superficial zone of human meniscus. However, no collagen type I staining was detected in pseudo-superficial zone of mouse meniscus. In addition, collagen type I staining was examined only in the outer part of the mouse meniscus. The MMPCs could be differentiated only into two mesenchymal linages, i.e., cells of the chondroblastic lineage and the osteoblastic lineage.

In brief, the main theme of this study was to introduce novel, until now uncharacterized MPCs and MMPCs as possible candidates for meniscus tissue regeneration.

In summary, the initial findings will contribute to broadening the concept of adult progenitor cells in situ, present in mature tissues. Furthermore, it will motivate the researcher to understand the biological nature of the progenitor cells, importantly, to establish mechanistic approaches to introduce progenitor cells as cellular therapeutics for meniscus regeneration. 


\section{Chapter 1}

Current concepts in stem cell therapy for articular cartilage repair

Hayat Muhammad ${ }^{1}$, Boris Schminke ${ }^{1}$ and Nicolai Miosge ${ }^{1 \#}$

${ }^{1}$ Georg August University, Tissue Regeneration Work Group

Department of Prosthodontics Goettingen, Germany

"Corresponding author

Hayat Muhammad's contribution: Literature search and writing of the manuscript. 


\section{EXPERT OPINION}

1. Introduction

2. Human embryonic stem cells and parental stem cells as candidates for cartilage repair

3. Adult stem cells as candidates for cartilage repair

4. Issue of stem cell transplantation and immune response

5. Induced pluripotent stem cells as candidates for cartilage repair

6. Progenitor cells as candidates for cartilage regeneration

7. CPCs might be suitable for cartilage repair in situ but still face limitations

8. Expert opinion

\section{informa}

\section{Current concepts in stem cell therapy for articular cartilage repair}

\author{
Hayat Muhammad, Boris Schminke \& Nicolai Miosge ${ }^{\dagger}$ \\ Georg August University, Tissue Regeneration Work Group, Department of Prosthodontics, \\ Goettingen, Germany
}

Introduction: Hyaline articular cartilage is the connective tissue responsible for frictionless joint movement. Its degeneration ultimately results in complete loss of joint function in the late stages of osteoarthritis. Intrinsic repair is compromised, and cartilage tissue regeneration is difficult. However, new options are available to repair cartilage tissue by applying ESCs, MSCs and CPCs.

Areas covered: In this review, the authors shed light on the different concepts currently under investigation for cartilage repair.

Expert opinion: So far, there is no way to derive a chondrogenic lineage from stem cells that forms functional hyaline cartilage tissue in vivo. One alternative might be to enhance the chondrogenic potential of repair cells, which are already present in diseased cartilage tissue. CPCS found in diseased cartilage tissue in situ are biologically driven toward the osteochondrogenic lineage and can be directed toward chondrogenesis at least in vitro.

Keywords: cartilage, chondrogenic progenitor cells, collagens, extracellular matrix, osteoarthritis, sox9/runx2, stem cells

Expert Opin. Biol. Ther. (2013) 13(4):541-548

\section{Introduction}

The progression of knee osteoarthritis (OA) is multigenic in origin [1,2]. In addition, gender has a powerful influence on the incidence, severity and pathogenesis of $\mathrm{OA}$ [3]. Once damaged or injured, articular cartilage has a limited intrinsic capability for self-repair because of its avascular nature [4]. For regeneration, two major concerns need to be discussed: first, how to heal or fill the affected area with a tissue that has the same biological and mechanochemical properties as articular cartilage; and second, how to enhance and promote successful integration between the new repair tissue and the native articular cartilage [5]. Articular cartilage is responsible for the painless and frictionless movement of the joint; its degeneration leads to the exposure of the bone and this results in painful direct bone-to-bone contact. Fibrocartilaginous repair tissue is present in the late stages of $\mathrm{OA}$ and harbors diseased chondrocytes as well as elongated fibroblast-like cells (Figure 1). The authors found unique progenitor cells, termed chondrogenic progenitor cells (CPCs) within this repair tissue from human articular cartilage during the late stages of OA (Figure 2). These CPCs have a tremendous ability for chondrogenesis [6]. This review will discuss all cell-based therapeutic approaches to generate cartilaginous repair tissue that may ultimately lead to clinical applications.

\section{Human embryonic stem cells and parental stem cells as candidates for cartilage repair}

The tissue regeneration community shows a great interest in embryonic stem cells (ESCs) because of their unlimited self-renewal and pluripotency. They represent 

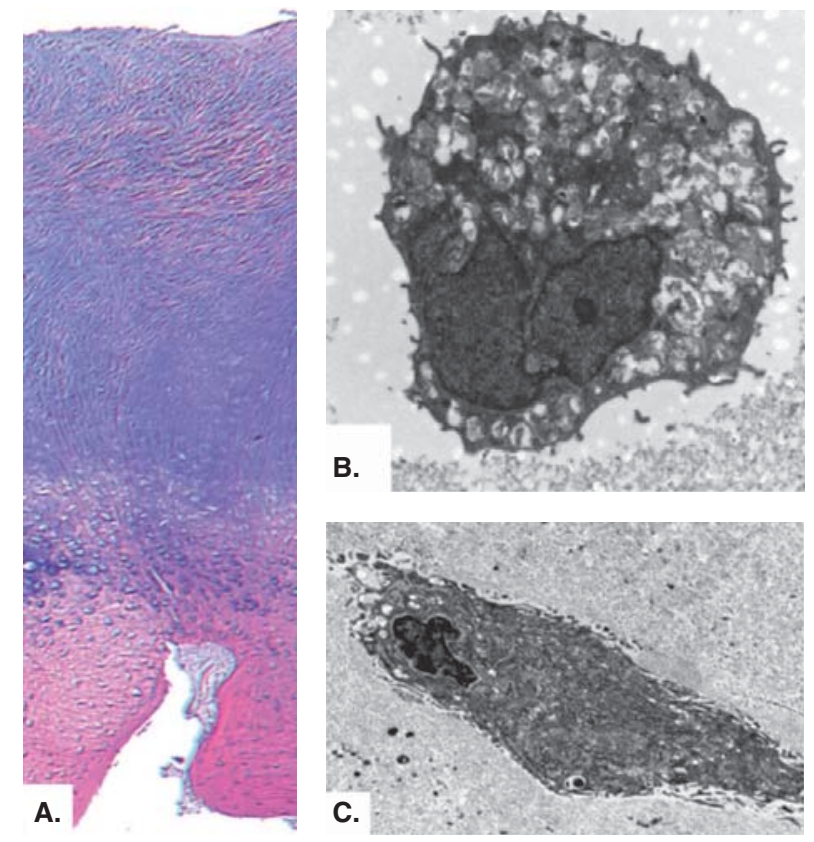

Figure 1. (A) Light microscopic histology of fibrocartilaginous tissue indicating regeneration attempts in the late stages of human osteoarthritis. (B) Electron micrograph of a diseased chondrocyte from the late stages of osteoarthritis and (C) a fibroblast-like cell from fibrocartilaginous tissue as shown in $\mathrm{A}$.

an immortal cell that could potentially provide an unlimited supply of any target tissue (except placental cells) for cell-based therapies.

ESCs, from the inner cell mass of the blastocyst can be isolated and propagated in vitro. Mouse ESCs were the first to be investigated in $1981[7,8]$. The next major breakthrough was in 1998 when Thomson et al. isolated ESCs from human embryos [9]. These cells have the potential to produce all or most cell lineages derived from the three embryonic germ layers: ectoderm (skin and neural lineages), mesoderm (blood, fat, cartilage, bone and muscle) and endoderm (the digestive and respiratory system) [10].

The development of an efficient and stable culture condition to drive differentiation to the desired tissue-specific cells is the crucial issue, because undifferentiated ESCs facilitate teratoma formation in vivo [11,12]. Although it is possible to enhance the differentiation potential toward a certain cell type [13,14], obtaining a homogeneously purified population is still challenging. Therefore, there is a need for further research to understand and optimize different factors, signals and environmental influences that induce and restrict human ESCs to differentiate specifically toward the chondrogenic lineage.

It is not trivial to bypass embryonic body (EB) formation and initiate the direct conversion of ESCs into chondrocytes. Yamashita et al. reported the direct chondrogenic differentiation of mouse ESCs using high-density adherent micromass culture and $1 \%$ fetal bovine serum (FBS). Under high-serum conditions, differentiation to multiple lineages has been observed [15]. A growth factor-directed chondrogenesis has also been demonstrated through the application of cocktails containing different growth factors in varying proportions [16]. Growth factors of the TGF- $\beta$ family are considered to be potent inducers of the chondrogenic differentiation of ESCs, and in the combination with bone morphogenic protein (BMP)-7 these growth factors facilitate chondrogenesis without EB formation [17,18]. Human ESCs have been reported to differentiate successfully into chondrocytes using co-culture and scaffold differentiation methods, and these differentiated chondrocytes have been tested in vivo for cartilage repair in mouse models without teratoma formation [19-22]. Nevertheless, any application of ESCs in humans harbors the risk of teratoma formation.

Prenatal tissues, such as human umbilical cord (Wharton's jelly), cord blood and amnion all harbor stem cells expressing mesenchymal stem cells (MSCs)-related markers. Although most studies have mainly been concerned with osteogenic lineage differentiation, the capacity to differentiate into the chondrogenic lineage has also been observed with these parental stem cells. Expression of the main extracellular matrix (ECM) chondrogenic markers, including collagen II, has been demonstrated in these cells [23-33]. More recently, de Mara et al. isolated MSCs from human umbilical blood cells, and their chondrogenesis was enhanced, using the BMP-2 and BMP-6 proteins [34]. The potential of human umbilical cord (Wharton's jelly) stem cells is enhanced when cultured on nanofibrous scaffolds [35].

\section{Adult stem cells as candidates for cartilage repair}

Adult stem cells are progenitor cells, which reside in already differentiated tissues. Many adult tissues exhibit a certain capacity for self-renewal after trauma or disease. For instance, bone marrow is the major pool of adult hematopoietic stem cells (HSCs) that replenish circulating blood cells [36]. Wakitani et al. performed the first transplantation of bone marrow-derived MSCs for cartilage repair, and a sort of cartilage-like tissue was observed after 42 weeks [37]. Like HSCs, MSCs are also believed to reside in the bone marrow. They take part in the regeneration of mesenchymal tissues, such as bone, cartilage, muscle, ligament, tendon, adipose and stroma [38-47]. Pittenger et al. first isolated adult MSCs from bone marrow and demonstrated their multilineage differentiation potential [48]. Subsequently, researchers isolated MSCs from various other adult mesenchymal tissues, such as blood, adipose, skin, mandible trabecular bone, muscle and synovial membrane [49]. The diversity of the chondrogenic potential of MSCs isolated from these different tissues is still being investigated. However, it has been suggested that MSCs derived from adipose tissue have the highest chondrogenic potential [50]. More recently, Suzuki et al. reported that aggregated synovial MSCs are a better choice for cartilage repair. These cells exhibit 

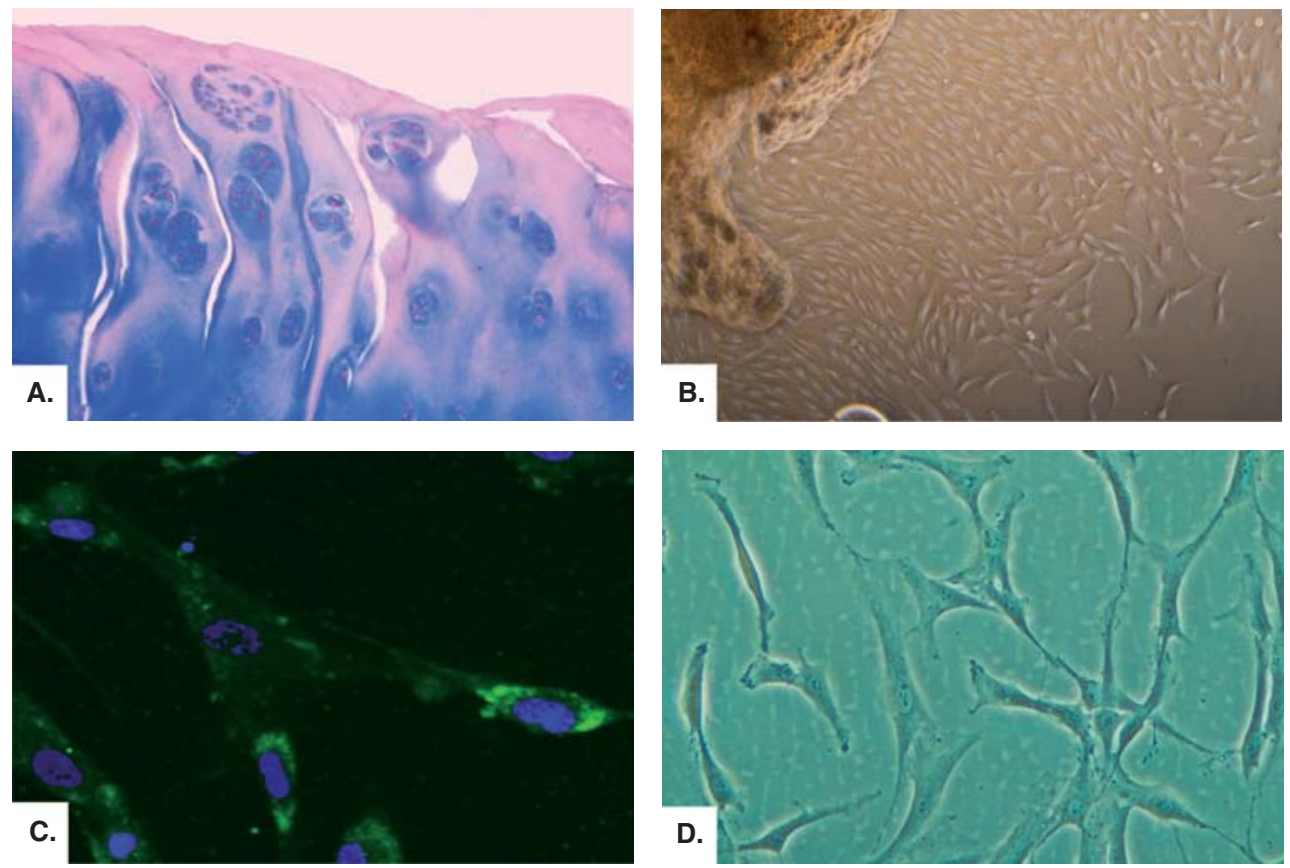

Figure 2. (A) Light microscopic picture of cluster formation as a sign of regeneration found in the late stages of osteoarthritis. (B) Cells migrating out of a sample of fibrocartilaginous tissue after 10 days in culture. (C) These cells are STRO-1 positive and (D) show a fibroblast-like phenotype and have been characterized as CPCs [6].

a high expansion capacity, efficient attachment and a high chondrogenic potential [51]. An increased number of MSCs are observed in the synovial fluid of patients with OA [52]. Applying small inhibitory agents could enhance the chondrogenic potential of human MSCs. Johnson et al. identified a small molecule that promotes chondrogenic differentiation in human MSCs. The inhibitor kartogenin binds filamin A, inhibits its binding to the transfection factor core-binding factor $\beta$-subunit and enhances chondrogenesis by regulating the core-binding factor $\beta$ that suppresses runx 2 expression [53]. All of these experiments that describe new enhancement methods for the chondrogenesis of MSCs indicate that no single method is yet efficient for cartilage tissue regeneration. Research has yet to even elucidate the basic cellular mechanisms of MSC differentiation into chondrocytes.

Stem cells, once isolated, purified and completely detached from their tissue-specific environment, do not behave as they would in vivo. In 1978, Schofield introduced the concept that stem cells are located in particular anatomical sites in adult tissues, called niches [54]. A niche is a stem cell's surrounding microenvironment, including its ECM and helper cells. Stem cells live in their niche for an indefinite period of time and self-renew from time to time to release a transient amplifying pool of cells (Figure 3). The components of this environment, for example, the stem cells themselves, the niche cells, the ECM and secreted proteins, are coordinated to maintain the stem cell phenotype and direct the daughter cells to differentiate into cells of the transient amplifying pool to escape from the niche [55]. It is crucial to understand the main fundamental signals and pathways that a stem cell receives when it is located in its niche, as this signaling information will help to maintain these cells in an undifferentiated state in an artificial in vitro environment.

\section{Issue of stem cell transplantation and immune response}

Adult MSCs are believed to be immunosuppressive [56]. Sato et al. injected human MSCs into the osteoarthritic knee joint of guinea pigs and demonstrated that MSCs integrate into a tissue without any immunogenic response [57]. Major histocompatibility (MHC) antigens on the donor cells and their exposure to the host normally results in a biological rejection. Fortunately, the number of MHC antigens is low on undifferentiated cells. However, the MHC level increases on differentiation [58]. MHC antigen expression is also very low on human chondrocytes. Usually, human chondrocytes express the class I MHC and do not normally express the class II MHC [59]. Therefore, immunoreactions are unlikely; however, some authors believe that a potential immunesuppressive drug therapy will be required at least for ESC-based application [60,61].

\section{Induced pluripotent stem cells as candidates for cartilage repair}

Induced pluripotent stem cells (iPSCs) offer an allogeneic cell source for cartilage repair. Because they are obtained from 
the patient's own body, they could provide a patient-specific cell-mediated therapy [62,63]. Nuclear transfer experiments have confirmed that already differentiated cells can be reprogrammed to form an entire organism. Takahashi and Yamanaka pioneered the concept that the expression of only four transcription factors is sufficient to render mammalian somatic cells pluripotent. Further research developments have reduced the number of transcription factors to only oct 4 , which is at least sufficient for the induction of pluripotency in neural stem cells. In contrast to somatic cells, germline cells express oct 4 to retain their pluripotency. Therefore, Sterneckert et al. suggest that oct 4 is the gatekeeper for the reprogramming expressway [64,65].

Kim et al. generated iPSCs from OA-patient-derived synovial cells. Four well-known transcription factors (oct4, sox2, klf4 and c-myc) were introduced into the cells to induce reprogramming; the cells were then subjected to chondrogenic differentiation. These experiments suggest that iPSCs exert a potential for cellbased cartilage repair [66]. Recently, Hiramatsu et al. generated hyaline cartilaginous tissue from mouse adult dermal fibroblast cultures using only two reprogramming factors (c-myc and klf4) and one chondrogenic factor (sox9) [67]. Adult somatic cells reprogrammed via nuclear transfer to acquire pluripotency retain the epigenetic memory of their tissue origin. Neural progenitor- and fibroblast-derived iPSCs retain residual methylations at loci required for the hematopoietic fate, so their blood-forming capacity is reduced in vitro [68].

There are risks associated with the clinical application of these cells because iPSCs are often generated using retroviruses, which can cause a lethal insertional mutagenesis and may result in adverse effects, such as those observed in gene therapy [69]. Mice grown from retrovirally derived iPSCs seem to be normal only under conditions in which the c-myc transgene is repressed [70]. The long-term safety of human iPSCs cannot be guaranteed through mouse experiments alone. The retroviruses applied to create iPSCs render them immunogenic [71]. For the clinical application of these cells, researchers should avoid the integration method. There are safer methods, such as using plasmids and Sendai virus [72,73]; however, all of these methods are still in the early experimental phase. A recent review published by Herberts et al. provides a comprehensive overview of all the risk factors, such as transformation, tumor formation and genetic modification that have to be faced in association with stem cell therapy [74].

\section{Progenitor cells as candidates for cartilage regeneration}

The authors have isolated migratory progenitor cell populations from late-stage human $\mathrm{OA}$ tissue. These cells are positive for the well-known stem cells markers CD105, CD106, CD73, CD29 and Stro-1. However, these cells were negative for the hematopoietic markers, such as CD45. CPCs are multipotent and exhibit a tremendous chondrogenic potential [6]. The knockdown of runx 2 enhances the chondrogenic potential of CPCs by the up-regulation of sox 9 and collagen II expression (Figure 3). Recently it was shown that CPCs from both genders were influenced by sex hormones. Both estrogen receptors (ER) $\alpha$ and $E R \beta$ and androgen receptors were detected on CPCs. The presence of sex steroids in the synovial fluid and the modulatory effect of estrogen on CPCs might be of relevance for joint biology and stem cell-based therapy. The cells of human OA cartilage were partially positive for the sex hormone receptors, while all CPC populations were found to be highly positive for these receptors. The chondrogenic potential was enhanced by treating the female-derived CPCs with $17 \beta$-estradiol and treating the male-derived CPCs with testosterone [75]. The osteogenic differentiation of murine ESCs is negatively regulated by estrogen [76]. Jung et al. have demonstrated that estrogen helps to maintain stemness and oct 4 expression to keep the ESCs in an undifferentiated state [77]. Mouse ESCs were rescued from oxidative stress by dihydroxy testosterone [78], and Hong et al. recently found that estrogen induced the proliferation of MSCs [79].

\section{CPCs might be suitable for cartilage repair in situ but still face limitations}

The progenitor cell studies produced exciting data revealing the potential for the future clinical application of CPCs for cartilage repair [6,75]. However, further research is required before a clinical application can be developed:

1) A strong influence of age, gender and body weight on the regenerative potential of CPCs has to be taken into consideration.

2) Further questions remain regarding the guidance of these cells to the diseased area. Additionally, the degenerated and inflamed ECM has a negative effect on the repair potential of CPCs. In particular, cytokines that are responsible for OA initiation [80] might negatively influence the chondrogenic potential of CPCs.

3) The proposal of a safe method, condition or agent that drives the CPCs in situ to entirely replenish native cartilage with collagen type II synthesis, rather than the fibrocartilaginous tissue with high amounts of collagen type I, remains to be established.

4) It is necessary to elucidate the role of the stem cell niche, from which the CPCs are derived, to learn more about the microenvironment and how this influences the niche and the progenitor cell release.

5) In situ manipulation of these cells will be crucial, because CPCs are progenitor cells and not stem cells and, therefore, reach senescence in cell culture.

\section{Expert opinion}

The main aim of tissue regenerative therapies is to replace the damaged, injured or diseased tissue with proper native-like 


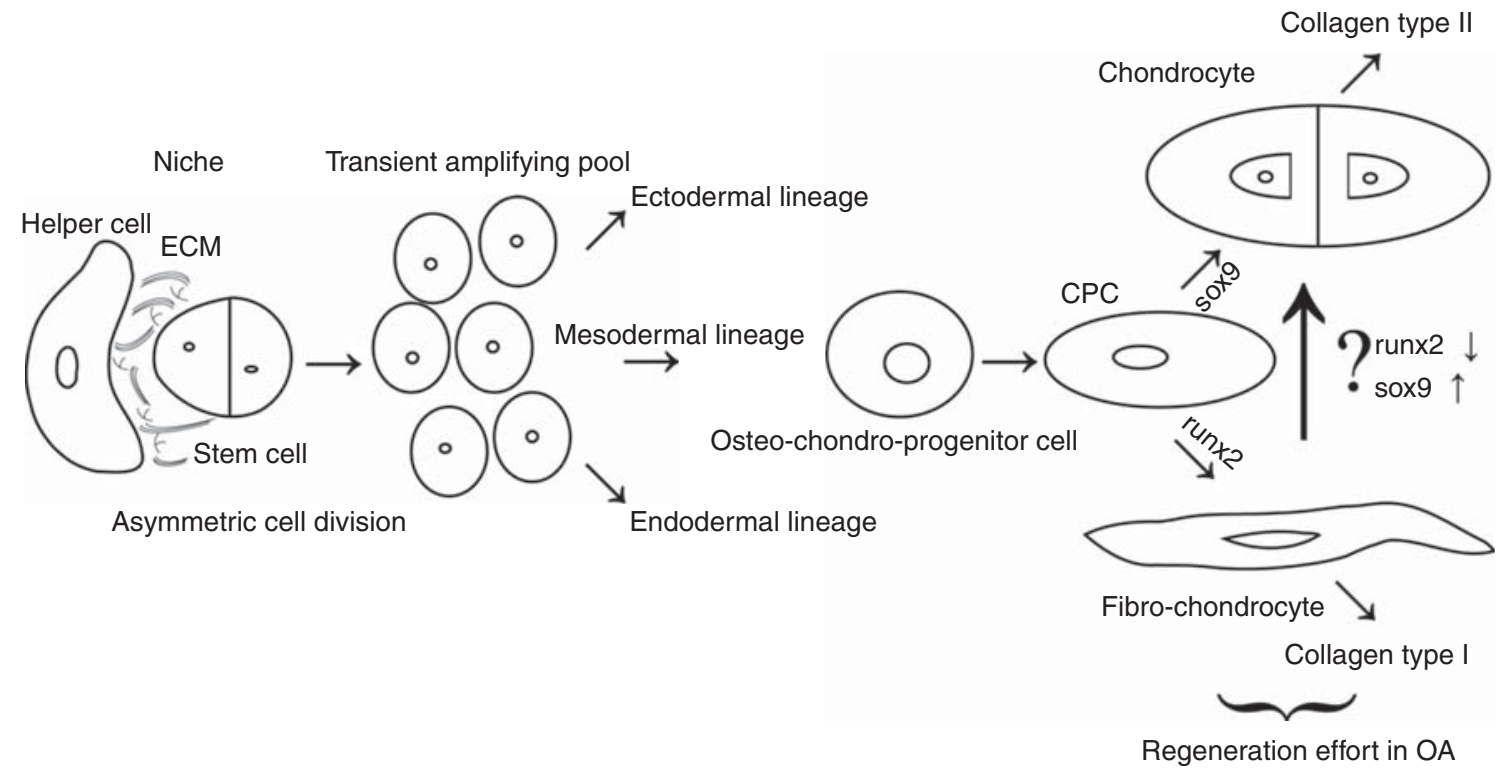

Figure 3. The image depicts the concept of progenitor cells in situ. The stem cell resides in its niche and releases cells of the transient amplifying pool, which, in the case of cartilage repair, develop into osteochondral progenitor cells. These cells in turn become chondrogenic progenitor cells found in the repair tissue that produces collagen type I. The aim would be to manipulate these cells in situ to differentiate them into chondrocytes to produce collagen type II. The hypothesis is that this can be achieved via runx2 and sox9.

Table 1. Various types of progenitor/stem cells under investigation for cartilage repair.

\begin{tabular}{|c|c|c|c|c|}
\hline Cell types & ESCs & iPSCs & MSCs & $\begin{array}{l}\text { In situ progenitor } \\
\text { cells (CPCs) }\end{array}$ \\
\hline Origin & $\begin{array}{l}\text { Derived from inner cell mass } \\
\text { of blastocyst }\end{array}$ & $\begin{array}{l}\text { Derived from somatic cells } \\
\text { via stemness factors }\end{array}$ & Isolated from adult tissue & Isolated from OA cartilage \\
\hline Self-renewal & Self-renewal & Self-renewal & $\begin{array}{l}\text { Somewhat limited self- } \\
\text { renewal }\end{array}$ & Limited self-renewal \\
\hline Life span & Indefinite & Indefinite & Limited life span & $\begin{array}{l}\text { Limited life span, less than } \\
\text { MSCs }\end{array}$ \\
\hline $\begin{array}{l}\text { Teratoma } \\
\text { formation }\end{array}$ & High teratoma risk & High teratoma risk & No teratoma risk & No teratoma risk \\
\hline $\begin{array}{l}\text { Differentia- } \\
\text { tion } \\
\text { potential }\end{array}$ & Pluripotent & Pluripotent & Multipotent & Multipotent \\
\hline $\begin{array}{l}\text { Preclinical } \\
\text { aspects }\end{array}$ & $\begin{array}{l}\text { Can differentiate in all cell } \\
\text { types of the three germ } \\
\text { layers }\end{array}$ & $\begin{array}{l}\text { Can potentially differentiate } \\
\text { in all cell types of the three } \\
\text { germ layers }\end{array}$ & $\begin{array}{l}\text { Can differentiate mainly into } \\
\text { the cell types of the } \\
\text { mesodermal lineage }\end{array}$ & $\begin{array}{l}\text { Already determined to the } \\
\text { osteochondrogenic lineage }\end{array}$ \\
\hline Immunity & $\begin{array}{l}\text { Low level of MHC I and II, } \\
\text { less immunogenic }\end{array}$ & $\begin{array}{l}\text { Normal level of } \mathrm{MHC} \text { I and II, } \\
\text { highly immunogenic }\end{array}$ & Less immunogenic & Not noticed until now \\
\hline $\begin{array}{l}\text { Preclinical } \\
\text { aspect }\end{array}$ & $\begin{array}{l}\text { Many steps required to } \\
\text { obtain the desired cell type }\end{array}$ & $\begin{array}{l}\text { Many steps required to } \\
\text { obtain the desired cell type }\end{array}$ & $\begin{array}{l}\text { Difficult to maintain } \\
\text { undifferentiated in cell } \\
\text { culture }\end{array}$ & $\begin{array}{l}\text { Easy to isolate and } \\
\text { differentiate into } \\
\text { chondrocytes }\end{array}$ \\
\hline
\end{tabular}

ESC: Embryonic stem cell; iPSCs: Induced pluripotent stem cells; MSC: Mesenchymal stem cell; OA: Osteoarthritis.

functional tissue. However, the well-known physiological repair mechanisms associated with diseased cartilage are rare and generally overridden by matrix destruction that results in less functional fibrocartilaginous tissue, with more collagen type I and less collagen type II [81,82]. Stem cells are viewed as one of the best candidates for cartilage repair. To address these challenges, researchers have tested the chondrogenic potential of a broad-spectrum of multipotent stem cells such as adult MSCs, ESCs and iPSCs (Table 1). Comparative studies have demonstrated that each cell type has advantages and 
disadvantages. The published data also demonstrated that some sources may be more useful than others and more promising for cartilage repair [83]. As of now, none of the published successes in driving stem cells into the chondrogenic lineage has resulted in functional hyaline cartilage tissue. Furthermore, the long-term effects in these cells such as ESCs-driven teratoma formation, the imprinted genetic memory of iPSCs and the insertional mutagenesis of retroviruses used to induce iPSCs should be examined. Stem cell differentiation potential decreases with age; and $\mathrm{OA}$ is a disease of the elderly. The MSCs from aged OA patients have a very low differentiation potential for cartilage-like tissue formation [84]. More recently, patients from moderate to late stages of OA were subjected to MSCs treatment, but no complete regeneration of cartilage and no long-term satisfactory results were obtained [85]. Thus, another concept in regenerative medicine is to regard MSCs more as a therapeutic means to stimulate the stem cells already present in the diseased tissue and guide these stem cells toward regeneration activities, instead of transplanting cells to regenerate the tissue de novo. Therefore, the authors suggest that resident cells in diseased cartilage tissue, that are already present in situ and are active as a physiological response to the cell biological stimuli of the diseased tissue should be taken into consideration. They might be more efficient and safer than exogenous cells. The authors' research group aims mainly to elucidate the chondrogenic pathways in CPCs and to investigate the interacting partners of two of their master regulators, sox 9 and runx2. However, before a biological therapy of OA with CPCs becomes a clinical reality, numerous questions, as outlined in Section 7, remain to be addressed.

\section{Declaration of interest}

The authors would like to thank the members of their group for helpful discussions and the DFG for funding.

\section{Bibliography}

1. Valdes AM, Hart DJ, Jones KA, et al. Association study of candidate genes for the prevalence and progression of knee osteoarthritis. Arthritis Rheum 2004;50(8):2497-507

2. Valdes AM, Van Oene M, Hart DJ, et al. Reproducible genetic associations between candidate genes and clinical knee osteoarthritis in men and women. Arthritis Rheum 2006;54(2):533-9

3. Richette P, Corvol M, Bardin T. Estrogens, cartilage, and osteoarthritis. Joint Bone Spine 2003;70(4):257-62

4. Malda J, Kreijveld E, Temenoff JS, et al. Expansion of human nasal chondrocytes on macroporous microcarriers enhances redifferentiation. Biomaterials 2003;24(28):5153-61

5. Redman SN, Oldfield SF, Archer CW. Current strategies for articular cartilage repair. Eur Cell Mater 2005;9:23-32; discussion 23-32

6. Koelling S, Kruegel J, Irmer M, et al. Migratory chondrogenic progenitor cells from repair tissue during the later stages of human osteoarthritis. Cell Stem Cell 2009;4(4):324-35

7. Evans MJ, Kaufman MH. Establishment in culture of pluripotential cells from mouse embryos. Nature 1981;292(5819):154-6

8. Martin GR. Isolation of a pluripotent cell line from early mouse embryos cultured in medium conditioned by teratocarcinoma stem cells.

Proc Natl Acad Sci USA

1981;78(12):7634-8

9. Thomson JA, Itskovitz-Eldor J, Shapiro SS, et al. Embryonic stem cell lines derived from human blastocysts. Science 1998;282(5391):1145-7

10. Gardner RL, Beddington RS. Multi-lineage 'stem' cells in the mammalian embryo. J Cell Sci Suppl 1988;10:11-27

11. Toh WS, Lee EH, Cao T. Potential of human embryonic stem cells in cartilage tissue engineering and regenerative medicine. Stem Cell Rev 2011;7(3):544-59

12. Heng BC, Cao T, Lee EH. Directing stem cell differentiation into the chondrogenic lineage in vitro. Stem Cells 2004;22(7):1152-67

13. Kawaguchi J, Mee PJ, Smith AG. Osteogenic and chondrogenic differentiation of embryonic stem cells in response to specific growth factors. Bone 2005;36(5):758-69

14. zur Nieden NI, Kempka G, Rancourt DE, Ahr HJ. Induction of chondro-, osteo- and adipogenesis in embryonic stem cells by bone morphogenetic protein-2: effect of cofactors on differentiating lineages. BMC Dev Biol 2005;5:1

15. Yamashita A, Krawetz R, Rancourt DE. Loss of discordant cells during micro-mass differentiation of embryonic stem cells into the chondrocyte lineage. Cell Death Differ 2009;16(2):278-86

16. Toh WS, Yang Z, Liu H, et al. Effects of culture conditions and bone morphogenetic protein 2 on extent of chondrogenesis from human embryonic stem cells. Stem Cells 2007;25(4):950-60

17. Nakagawa T, Lee SY, Reddi AH. Induction of chondrogenesis from human embryonic stem cells without embryoid body formation by bone morphogenetic protein 7 and transforming growth factor beta1. Arthritis Rheum 2009;60(12):3686-92

18. Yang Z, Sui L, Toh WS, et al. Stage-dependent effect of TGF-betal on chondrogenic differentiation of human embryonic stem cells. Stem Cells Dev 2009; 18(6):929-40

19. Vats A, Bielby RC, Tolley N, et al. Chondrogenic differentiation of human embryonic stem cells: the effect of the micro-environment. Tissue Eng 2006;12(6):1687-97

20. Hwang NS, Varghese $S$, Elisseeff J. Derivation of chondrogenically-committed cells from human embryonic cells for cartilage tissue regeneration. PLoS ONE 2008;3(6):e2498

21. Bai HY, Chen GA, Mao GH, et al. Three step derivation of cartilage like tissue from human embryonic stem cells by $2 \mathrm{D}-3 \mathrm{D}$ sequential culture in vitro and further implantation 
in vivo on alginate/PLGA scaffolds. J Biomed Mater Res A 2010;94(2):539-46

22. Hwang NS, Varghese S, Lee HJ, et al. In vivo commitment and functional tissue regeneration using human embryonic stem cell-derived mesenchymal cells. Proc Natl Acad Sci USA 2008;105(52):20641-6

23. Sarugaser R, Lickorish D, Baksh D, et al. Human umbilical cord perivascular (HUCPV) cells: a source of mesenchymal progenitors. Stem Cells 2005;23(2):220-9

24. Wang HS, Hung SC, Peng ST, et al. Mesenchymal stem cells in the Wharton's jelly of the human umbilical cord. Stem Cells 2004;22(7):1330-7

25. Erices A, Conget P, Minguell JJ. Mesenchymal progenitor cells in human umbilical cord blood. Br J Haematol 2000;109(1):235-42

26. Rosada C, Justesen J, Melsvik D, et al. The human umbilical cord blood: a potential source for osteoblast progenitor cells. Calcif Tissue Int 2003;72(2):135-42

27. Gang EJ, Hong SH, Jeong JA, et al. In vitro mesengenic potential of human umbilical cord blood-derived mesenchymal stem cells. Biochem Biophys Res Commun 2004;321(1):102-8

28. Kogler G, Sensken S, Airey JA, et al. A new human somatic stem cell from placental cord blood with intrinsic pluripotent differentiation potential. J Exp Med 2004;200(2):123-35

29. Lee OK, Kuo TK, Chen WM, et al. Isolation of multipotent mesenchymal stem cells from umbilical cord blood. Blood 2004;103(5):1669-75

30. Tondreau T, Meuleman N, Delforge A, et al. Mesenchymal stem cells derived from CD133-positive cells in mobilized peripheral blood and cord blood: proliferation, Oct4 expression, and plasticity. Stem Cells 2005;23(8):1105-12

31. Romanov YA, Svintsitskaya VA, Smirnov VN. Searching for alternative sources of postnatal human mesenchymal stem cells: candidate MSC-like cells from umbilical cord. Stem Cells 2003;21(1):105-10

32. Kim JW, Kim SY, Park SY, et al. Mesenchymal progenitor cells in the human umbilical cord. Ann Hematol 2004;83(12):733-8

33. De Coppi P, Bartsch G Jr, Siddiqui MM, et al. Isolation of amniotic stem cell lines with potential for therapy. Nat Biotechnol 2007;25(1):100-6

34. de Mara CS, Duarte AS, Sartori-Cintra AR, et al. Chondrogenesis from umbilical cord blood cells stimulated with BMP-2 and BMP-6. Rheumatol Int 2012

35. Fong CY, Subramanian A, Gauthaman K, et al. Human umbilical cord Wharton's jelly stem cells undergo enhanced chondrogenic differentiation when grown on nanofibrous scaffolds and in a sequential two-stage culture medium environment. Stem Cell Rev 2012;8(1):195-209

36. Till JE, Mc CE. A direct measurement of the radiation sensitivity of normal mouse bone marrow cells. Radiat Res 1961;14:213-22

37. Wakitani S, Imoto K, Yamamoto T, et al. Human autologous culture expanded bone marrow mesenchymal cell transplantation for repair of cartilage defects in osteoarthritic knees.

Osteoarthritis Cartilage 2002;10(3):199-206

38. Kuznetsov SA, Krebsbach PH, Satomura K, et al. Single-colony derived strains of human marrow stromal fibroblasts form bone after transplantation in vivo. J Bone Miner Res 1997;12(9):1335-47

39. Prockop DJ. Marrow stromal cells as stem cells for nonhematopoietic tissues. Science 1997;276(5309): $71-4$

40. Pereira RF, Halford KW, O'Hara MD, et al. Cultured adherent cells from marrow can serve as long-lasting precursor cells for bone, cartilage, and lung in irradiated mice. Proc Natl Acad Sci USA 1995;92(11):4857-61

41. Caplan AI. Mesenchymal stem cells. J Orthop Res 1991;9(5):641-50

42. Friedenstein AJ. Precursor cells of mechanocytes. Int Rev Cytol 1976; $47: 327-59$

43. Owen M, Friedenstein AJ. Stromal stem cells: marrow-derived osteogenic precursors. Ciba Found Symp 1988;136:42-60
44. Friedenstein AJ, Chailakhyan RK, Gerasimov UV. Bone marrow osteogenic stem cells: in vitro cultivation and transplantation in diffusion chambers. Cell Tissue Kinet 1987;20(3):263-72

45. Ashton BA, Allen TD, Howlett CR, et al. Formation of bone and cartilage by marrow stromal cells in diffusion chambers in vivo. Clin Orthop Relat Res 1980;151):294-307

46. Bab I, Howlett CR, Ashton BA, Owen ME. Ultrastructure of bone and cartilage formed in vivo in diffusion chambers. Clin Orthop Relat Res 1984;187):243-54

47. Haynesworth SE, Goshima J, Goldberg VM, Caplan AI.

Characterization of cells with osteogenic potential from human marrow. Bone 1992;13(1):81-8

48. Pittenger MF, Mackay AM, Beck SC, et al. Multilineage potential of adult human mesenchymal stem cells. Science 1999;284(5411):143-7

49. Punwar S, Khan WS. Mesenchymal stem cells and articular cartilage repair: clinical studies and future direction. Open Orthop J 2011;5(Suppl 2):296-301

50. Estes BT, Diekman BO, Gimble JM, Guilak F. Isolation of adipose-derived stem cells and their induction to a chondrogenic phenotype. Nat Protoc 2010;5(7):1294-311

51. Suzuki S, Muneta T, Tsuji K, et al. Properties and usefulness of aggregates of synovial mesenchymal stem cells as a source for cartilage regeneration. Arthritis Res Ther 2012;14(3):R136

52. Sekiya I, Ojima M, Suzuki S, et al. Human mesenchymal stem cells in synovial fluid increase in the knee with degenerated cartilage and osteoarthritis. J Orthop Res 2012;30(6):943-9

53. Johnson K, Zhu S, Tremblay MS, et al. A stem cell-based approach to cartilage repair. Science 2012;336(6082):717-21

54. Schofield R. The relationship between the spleen colony-forming cell and the haemopoietic stem cell. Blood Cells 1978;4(1-2):7-25

55. Ohlstein B, Kai T, Decotto E, Spradling A. The stem cell niche: theme and variations. Curr Opin Cell Biol 2004;16(6):693-9 
H. Muhammad et al.

56. English K, Mahon BP. Allogeneic mesenchymal stem cells: agents of immune modulation. J Cell Biochem 2011;112(8):1963-8

57. Sato M, Uchida K, Nakajima H, et al. Direct transplantation of mesenchymal stem cells into the knee joints of Hartley strain guinea pigs with spontaneous osteoarthritis. Arthritis Res Ther 2012;14(1):R31

58. Drukker M, Benvenisty N. The immunogenicity of human embryonic stem-derived cells. Trends Biotechnol 2004;22(3):136-41

59. Jobanputra P, Corrigall V, Kingsley G, Panayi G. Cellular responses to human chondrocytes: absence of allogeneic responses in the presence of HLA-DR and ICAM-1. Clin Exp Immunol 1992;90(2):336-44

60. Swijnenburg RJ, Tanaka M, Vogel H, et al. Embryonic stem cell immunogenicity increases upon differentiation after transplantation into ischemic myocardium. Circulation 2005;112(9 Suppl):I166-72

61. Lui KO, Waldmann H, Fairchild PJ. Embryonic stem cells: overcoming the immunological barriers to cell replacement therapy. Curr Stem Cell Res Ther 2009;4(1):70-80

62. Amabile G, Meissner A. Induced pluripotent stem cells: current progress and potential for regenerative medicine. Trends Mol Med 2009;15(2):59-68

63. Wu SM, Hochedlinger K. Harnessing the potential of induced pluripotent stem cells for regenerative medicine. Nat Cell Biol 2011;13(5):497-505

64. Sterneckert J, Hoing S, Scholer HR. Concise review: oct 4 and more: the reprogramming expressway. Stem Cells 2012;30(1):15-21

65. Takahashi K, Yamanaka S. Induction of pluripotent stem cells from mouse embryonic and adult fibroblast cultures by defined factors. Cell 2006;126(4):663-76

66. Kim MJ, Son MJ, Son MY, et al. Generation of human induced pluripotent stem cells from osteoarthritis patient-derived synovial cells. Arthritis Rheum 2011;63(10):3010-21

67. Hiramatsu K, Sasagawa S, Outani H, et al. Generation of hyaline cartilaginous tissue from mouse adult dermal fibroblast culture by defined factors. J Clin Invest 2011;121(2):640-57

68. Kim K, Doi A, Wen B, et al. Epigenetic memory in induced pluripotent stem cells. Nature 2010;467(7313):285-90

69. Hacein-Bey-Abina S, Von Kalle C, Schmidt M, et al. LMO2-associated clonal $\mathrm{T}$ cell proliferation in two patients after gene therapy for SCID-X1. Science 2003;302(5644):415-19

70. Aoi T, Yae K, Nakagawa M, et al. Generation of pluripotent stem cells from adult mouse liver and stomach cells. Science 2008;321(5889):699-702

71. Zhao T, Zhang ZN, Rong Z, Xu Y. Immunogenicity of induced pluripotent stem cells. Nature 2011 Jun 9;474(7350):212-15

72. Okita K, Matsumura Y, Sato Y, et al. A more efficient method to generate integration-free human iPS cells. Nat Methods 2011;8(5):409-12

73. Fusaki N, Ban H, Nishiyama A, et al. Efficient induction of transgene-free human pluripotent stem cells using a vector based on Sendai virus, an RNA virus that does not integrate into the host genome. Proc Jpn Acad Ser B Phys Biol Sci 2009;85(8):348-62

74. Herberts CA, Kwa MS, Hermsen HP. Risk factors in the development of stem cell therapy. J Transl Med 2011;9:29

75. Koelling S, Miosge N. Sex differences of chondrogenic progenitor cells in late stages of osteoarthritis. Arthritis Rheum 2010;62(4):1077-87

76. Tielens S, Wymeersch F, Declercq H, Cornelissen M. Effect of 17beta-estradiol on the in vitro differentiation of murine embryonic stem cells into the osteogenic lineage. In Vitro Cell Dev Biol Anim 2008;44(8-9):368-78

77. Jung EM, Choi KC, Yu FH, Jeung EB. Effects of 17beta-estradiol and xenoestrogens on mouse embryonic stem cells. Toxicol In Vitro 2010;24(6):1538-45

78. Lee MN, Lee SH, Lee MY, et al. Effect of dihydrotestosterone on mouse embryonic stem cells exposed to $\mathrm{H} 2 \mathrm{O} 2$-induced oxidative stress. J Vet Sci 2008;9(3):247-56

79. Hong L, Zhang G, Sultana H, et al. The effects of 17-beta estradiol on enhancing proliferation of human bone marrow mesenchymal stromal cells in vitro. Stem Cells Dev 2011;20(5):925-31

80. Goldring SR, Goldring MB. The role of cytokines in cartilage matrix degeneration in osteoarthritis. Clin Orthop Relat Res 2004;427 Suppl):S27-36

81. Sandell LJ. Modern molecular analysis of a traditional disease: progression in osteoarthritis. Arthritis Rheum 2007;56(8):2474-7

82. Tesche F, Miosge N. New aspects of the pathogenesis of osteoarthritis: the role of fibroblast-like chondrocytes in late stages of the disease. Histol Histopathol 2005;20(1):329-37

83. Beane OS, Darling EM. Isolation, characterization, and differentiation of stem cells for cartilage regeneration. Ann Biomed Eng 2012;40(10):2079-97

84. Murphy JM, Dixon K, Beck S, et al. Reduced chondrogenic and adipogenic activity of mesenchymal stem cells from patients with advanced osteoarthritis. Arthritis Rheum 2002;46(3):704-13

85. Davatchi F, Abdollahi BS, Mohyeddin M, et al. Mesenchymal stem cell therapy for knee osteoarthritis. Preliminary report of four patients. Int J Rheum Dis 2011;14(2):211-15

\section{Affiliation}

Hayat Muhammad, Boris Schminke \& Nicolai Miosge ${ }^{\dagger}$

${ }^{\dagger}$ Author for correspondence

Georg August University,

Tissue Regeneration Work Group,

Department of Prosthodontics,

Robert-Koch-Str. 40, 37075,

Goettingen, Germany

E-mail: nmiosge@gwdg.de 


\section{Chapter 2}

Primary cilium as dual sensor of mechanochemical signals in chondrocytes

Hayat Muhammad ${ }^{1 *}$, and Yoach Rais ${ }^{2^{*}}$, Nicolai Miosge ${ }^{1 *}$ and Efrat Monsonego Ornan ${ }^{2}$

${ }^{1}$ Tissue Regeneration Work Group, Department of Prosthodontics,

Georg August University, Robert-Koch-Str. 40,

37075 Goettingen, Germany

${ }^{2}$ Faculty of Agricultural, Food and Environmental Sciences,

Institute of Biochemistry and Nutrition, Hebrew University,

POB 12, 76100 Rehovot, Israel

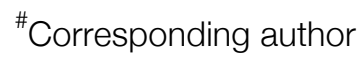

"Both authors contributed equally to this work

Hayat Muhammad's contribution: Literature search, immunofluorescence microscopy of primary cilia on CPCs and writing of manuscript. 


\title{
The primary cilium as a dual sensor of mechanochemical signals in chondrocytes
}

\author{
Hayat Muhammad • Yoach Rais • Nicolai Miosge • \\ Efrat Monsonego Ornan
}

Received: 24 August 2011/Revised: 18 December 2011/Accepted: 21 December 2011/Published online: 13 January 2012

(C) The Author(s) 2012. This article is published with open access at Springerlink.com

\begin{abstract}
The primary cilium is an immotile, solitary, and microtubule-based structure that projects from cell surfaces into the extracellular environment. The primary cilium functions as a dual sensor, as mechanosensors and chemosensors. The primary cilia coordinate several essential cell signaling pathways that are mainly involved in cell division and differentiation. A primary cilium malfunction can result in several human diseases. Mechanical loading is sense by mechanosensitive cells in nearly all tissues and organs. With this sensation, the mechanical signal is further transduced into biochemical signals involving pathways such as Akt, PKA, FAK, ERK, and MAPK. In this review, we focus on the fundamental functional and structural features of primary cilia in chondrocytes and chondrogenic cells.
\end{abstract}

Keywords Chondrocytes · Primary cilia .

Mechanotransduction - Chondorgenic progenitor cells

$\begin{array}{ll}\text { Abbreviations } \\ \text { OA } & \text { Osteoarthritis } \\ \text { ECM } & \text { Extracellular matrix } \\ \text { FAK } & \text { Focal adhesion kinase } \\ \text { MAPK } & \text { Mitogen-activated protein kinase }\end{array}$

H. Muhammad and Y. Rais contributed equally to this work.

H. Muhammad $\cdot$ N. Miosge $(\square)$

Tissue Regeneration Work Group, Department of

Prosthodontics, Georg August University, Robert-Koch-Str. 40,

37075 Goettingen, Germany

e-mail: nmiosge@gwdg.de

Y. Rais · E. M. Ornan

Faculty of Agricultural, Food and Environmental Sciences, Institute of Biochemistry and Nutrition, Hebrew University, POB 12, 76100 Rehovot, Israel

$\begin{array}{ll}\text { CPCs } & \text { Chondorgenic progenitor cells } \\ \text { ERK } & \text { Extracellular signal-regulated kinase } \\ \text { MSC } & \text { Mesenchymal stem cell } \\ \text { PKA } & \text { Protein kinase A } \\ \text { Hh } & \text { Hedgehog } \\ \text { IFT } & \text { Intraflagellar transport } \\ \text { Wnt } & \text { Wingless } \\ \text { EvC } & \text { Ellis-van Creveld syndrome } \\ \text { PKD } & \text { Polycystic kidney disease } \\ \text { Ihh } & \text { Indian Hedgehog }\end{array}$

\section{Introduction}

The morphological, structural, and material features of the cartilage are genetically programmed but can also be modified by epigenetic factors, such as local tissue stress and strain states [1, 2]. Mechanical stimulation resulting from weight loading, mobilization, and muscle contraction has an important role in bone formation and normal joint cavitation [3, 4]. Paralysis of the skeletal musculature is known to inhibit chondrogenesis in developing limbs [5], which thereby influences the length, mass, and mechanical properties of the forming bone [6-9]. The growth plate and articular cartilage are subjected to massive repeated mechanical forces, and they have a limited capacity for repair. Thus, understanding how articular cartilage is maintained and how mechanical loads are sensed by the chondrocytes is of primary importance.

\section{Sensing of mechanical signals}

Mechanosensitivity starts with external or internal mechanical responses, and the mechanical stimuli are transduced by 
the cell into a biochemical outcome. More precisely, this phenomenon is known as mechanochemical signaling or mechanotransduction. Multiple activation mechanisms are simultaneously at play, including the release of autocrine growth factors [10-17] activation of mechanically sensitive kinases, such as Src [18-22] focal adhesion kinase (FAK) [23-25] and extracellular-signal regulated kinase (ERK) [26-32] and initiation of second messenger signaling [33, 34]. Mechanical forces drive many cellular events, including proliferation, differentiation, and gene expression in adult differentiated cells and stem cells [35]. When trying to understand how cells can receive a variety of inputs and translate them into a response, we think of a system, or a cell organelle, that can perform these tasks. Interest in a specialized cell projection organ called the primary cilium has recently emerged. This organ was shown to have the ability to receive and transduce numerous cell signals [36]. Thus, the primary cilium is a good candidate to act as the cell's "control device" for mechanical stimulation because it projects as an "antenna" from the cell into the ECM, and it incorporates integrins, $\mathrm{G}$ protein receptors, and calcium channels into the cell membrane.

\section{Mechanosignaling in chondrocytes}

Ultrastructural studies have shown that each chondrocyte has such an immotile primary cilium. On chondrocytes, the primary cilia are oriented into the pericellular matrix environment of the chondron, and they interact with collagen types II and IV via receptors [37-42]. A physical and chemical deficiency in the chondroblastic and chondrocytic primary cilia results in skeletal and growth plate abnormalities due to improper ECM secretion [43-49]. Integrins, $\mathrm{G}$ proteins, and calcium channels on the primary cilium have all been implicated as mechanoreceptors [19, 50-53]. Numerous genes and pathways have been shown to be differentially regulated as a result of mechanical stimuli; for example, the phosphoinositide 3-kinase/Akt, protein kinase A (PKA) and Mitogen-activated protein kinase (MAPK) pathways [54-56]. It is reasonable to assume that mechanotransduction is a complex multi-component system that allows cells to integrate mechanical stimulations differing in intensity, frequency, duration, and orientation to generate appropriate biological responses, including cartilage formation and regeneration [57] and, especially, growth-plate formation [37, 58]. Mice and humans with mutations in ciliary genes often present with defects in skeletal development. Two human syndromes that include defects in endochondral bone formation were shown to be associated with mutations in ciliary genes. Asphyxiating thoracic dystrophy (Jeune's syndrome) is associated with a missense mutation in IFT80 (part of IFT complex B), and it presents with skeletal defects resembling those seen in sonic hedgehog homolog depletion. Furthermore, Ptc1 (hh receptor) expression is downregulated in the IFT80 mutant, suggesting that alterations in $\mathrm{Hh}$ signaling and Ellis-van Creveld syndrome $(\mathrm{EvC})$ are characterized by numerous skeletal and craniofacial abnormalities. The mutated protein in $\mathrm{EvC}$ has been localized to the base of the cilia expressed in chondrocytes and is required for normal $\mathrm{Hh}$ signaling. The disruption of $\mathrm{EvC}$ in mice resulted in a variety of skeletal abnormalities associated with diminished Ihh signaling [48].

Mice mutated in other ciliary genes also demonstrate alterations in endochondral ossification, resulting in a shortening of the long bones. Conditional deletion of IFT88 or KIF3a produces defects in embryonic endochondral bone formation, observed as early as 15.5 days from gestation [59]. These phenotypes resembled those seen in mice with germline mutations in Ihh [60]. When either Ift88 or KIF3a was deleted at later stages of development using the col2a-Cre promoter, the mice demonstrated a progressive loss of the cartilaginous growth plate, resulting in postnatal dwarfism that resembled the phenotype of mice with a conditional deletion of Ihh induced in postnatal cartilage. KIF3a and primary cilia are essential for coordination of chondrocytes maturation and condylar growth. The Ihh signaling pathway is one of the major regulatory pathways that lead to chondrocyte division and differentiation in the growth plate. Hydrostatic compression of the chondrocytic primary cilium upregulates Ihh gene expression [49, 61, 62].

Using electron microscopy, it has been shown that the chondrocyte cilium projects into the ECM and is tightly associated with the Golgi apparatus [39, 63]. Integrins have been shown to be present on the chondrocyte cilium, and integrin-dependent signaling cascades have been described in chondrocyte mechanotransduction [42, 64], suggesting a possible role for the chondrocytic cilia in mechanosensing. Chondrocytes in articular cartilage differ from those in the growth plate in that they are maintained as mature resting cells. Mechanical load is a critical factor in maintaining articular cartilage, but how the load is sensed is not known. Recently, the fate of the primary cilia on articular chondrocytes during the progression of bovine $\mathrm{OA}$ has been investigated $[47,65]$. Primary cilia were present during all examined stages of OA; however, the proportion of ciliated cells increased and their orientation towards the surface was altered; the significance of this orientation remains unclear [37, 63, 65]. Recently published cyclic compression experiments proved that mechanical loading modulates chondrocyte primary cilia incidence and length. This observation has been made independent from the well-known reduction of cilia appearance during cell division. Axonemal orientation in the cilia of articular 
chondrocytes is more pronounced in weight-bearing areas compared to of the cartilage tissue $[66,67]$.

We have studied the effect of loading on growth plate chondrocytes in vivo. Chondrocyte proliferation, differentiation, organization, and the major signaling pathways were found to be modified by loading in a chick model [68]. This demonstrated that the mechanical load affected chondrocytes in the growth plate [69], especially the expression of matrix metalloproteases [70].

\section{The primary cilium}

Primary cilia are non-motile sensory organelles that project from cells in many tissues and types of cells, such as kidney tubules, the bile duct, neurons, the endocrine pancreas, the thyroid, smooth muscle cells, and fibroblasts. The complete list of the cells and tissues containing primary cilia can be found at http://www.bowserlab.org/ primarycilia/cilialist.html. In recent years, cilia have emerged as a hot topic in research, resulting in the creation of numerous databases, including those containing genomic and proteomic data on cilium composition (http:// www.ciliaproteome.org, http://www.ciliome.com) [71-74]. Cilia can be seen as specialized cellular compartments or organelles [36, 75]. They are microtubule-based structures that originate at the basal body and extend into the extracellular space. The basal body is a modified form of the centriole, an organelle well known for its role as a microtubule organizing center of mitotic spindles. The basal body/centriole migrates toward the cell membrane and acts as a template for ciliogenesis and an anchor for the primary cilium. The centriole only moves towards the cell membrane when it is not involved in mitosis; thus, cilium generation occurs during the interphase of the cell. The intraflagellar transport (IFT) system plays a key role in primary cilium formation and maintenance. The IFT system is bidirectional and directs movement along the ciliary axoneme. Because no proteins are synthesized in the primary cilium itself, ciliary proteins are targeted to the basal body and transported to the distal tip through the IFT complex. IFT complexes are trafficked along the axoneme by molecular motor proteins; they are transported by kinesin II in the anterograde direction and by dynein II in the retrograde direction.

Motile and non-motile, primary cilia have microtubule axoneme cores made up of nine sets of microtubule doublets that provide structure and rigidity. However, primary cilia lack the central pair of microtubules (thus are designated $9+0$ ), while other motile machinery includes the inner and outer dynein arms, radial spokes, and central pair projections (designated 9+2). Unlike the motile cilia, of which there can be many per cell, there is only one primary cilium per cell [76-78].

\section{The function of the primary cilium}

To date, there are three hypotheses regarding the functional importance of the primary cilium: first, the primary cilium is a vestigial organ on the cell; second, that it inhibits cell division because it sequesters the centriole; and third, that it is a cellular sensory structure. The first hypothesis has been proven to be incorrect by several experiments. For instance, analyses of mutants, such as the $\mathrm{Tg} 737^{\text {orpk Rpw }}$ mouse, have indicated that a functioning primary cilium is essential for normal development and function, not only of the kidney, but also of many other tissues and organs. Hence, ciliary dysfunction might lead to a series of developmental abnormalities and diseases collectively called ciliopathies, including cystic diseases, obesity, and blindness, as well as behavioral, cognitive, and skeletal defects. The second hypothesis seems reasonable because the majority of cells possess primary cilia when they are not undergoing mitosis. Additionally, recent studies have demonstrated the accuracy of the third hypothesis: primary cilia have been shown to be highly involved in cell signaling processes because a number of ion channels, transporter proteins, and downstream effector proteins are associated with the cilium [79-81].

The primary cilium is a few micrometers in length, and it detects and interprets signals from the environment, such as odorants, fluid flow, and protein signaling between cells. Thus, they are spectacularly complex sensors. In ciliary signaling, the receptor protein and the protein that transmits the message into the cell are localized in the cilia. Protein association or dissociation from the cilia controls the signaling pathways, which ultimately trigger responses such as cell division and differentiation [82]. Several independent lines of evidence have demonstrated a role for the primary cilium in Hh signaling [83]. Hh is the master regulator of endochondral ossification in the growth plate, and it determines chondrocyte activity and subsequent bone length [84-86]. Upon Hh stimulation, both Hh receptors, Smo and ptch1, are recruited to the cilium in vitro and in vivo; Gli2 and Gli3, downstream effectors of $\mathrm{Hh}$, also localize to the cilium in the developing limbs [45, 87]. Other pathways that have been shown to regulate chondrocyte activity but have not yet been linked to the cilium are as follows: bone morphogenic proteins, wingless (Wnts), fibroblast growth factors, and insulin-like growth factors, all of which are essential for normal cartilage formation. 
Fig. 1 a Electron micrograph of the primary cilium (Ax), the distal (Dc), and proximal (Pc) centriole. Bar $500 \mathrm{~nm}$. b Cross section of the proximal centriole. Bar $100 \mathrm{~nm}$. Reprinted from Jensen et al. (1979) with permission.

c Schematic presentation of the primary cilium with membrane signaling molecules that were described in it. d Human articular chondrocytes and f CPCs stained with acetylated $\alpha$-tubulin (tb, green) Abs to detect primary cilia (arrows), phalloidin (ph, red) and DAPI (DAPI, blue). e Tissue from the late stage of human OA exhibits surface fissures and cell clusters (the arrow indicates the tidemark). Breaks in the tidemark are filled with blood vessels, and the bone marrow is visible underneath the $\mathrm{OA}$ tissue. Reprinted from Koelling et al. [92] with permission from the publisher
A

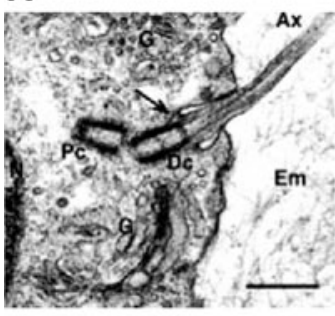

B

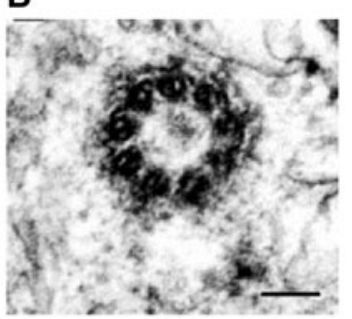

D

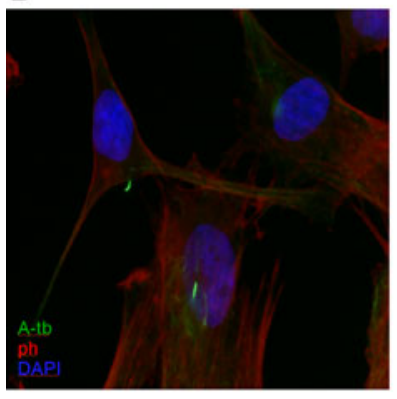

$F$

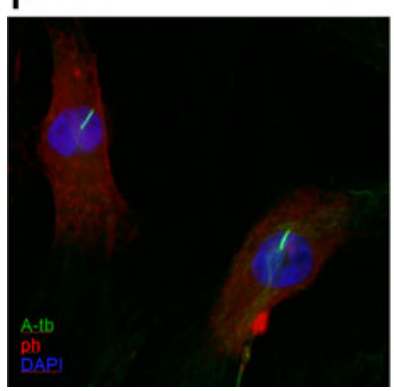

C

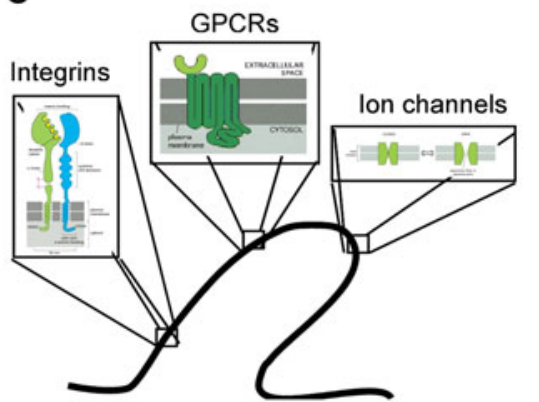

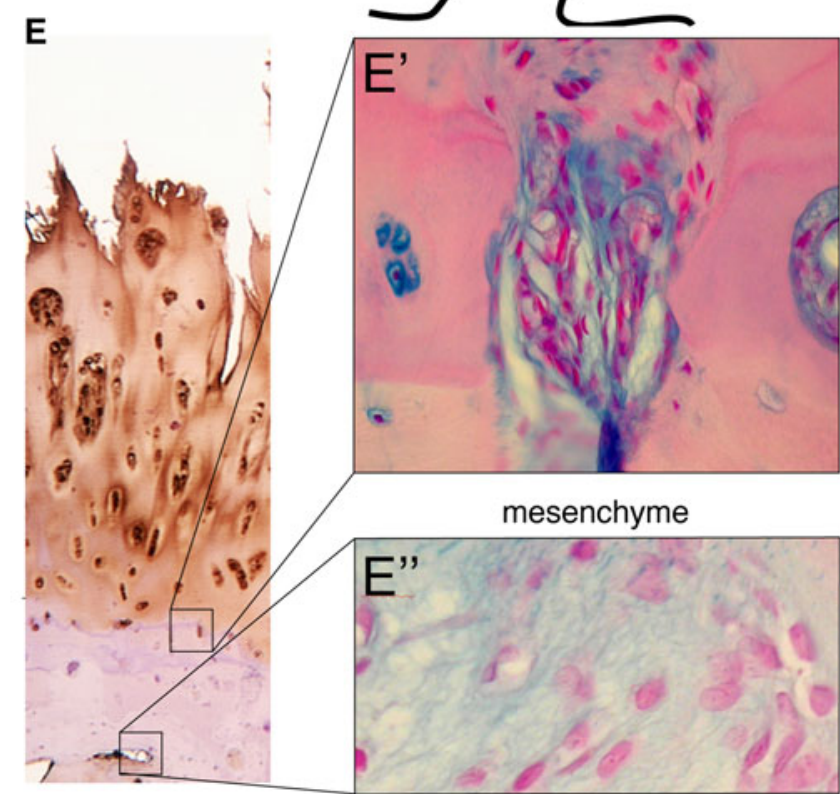

Cilia function in mesenchymal stem cells and chondrogenic progenitor cells (CPCs)

The effects of mechanical forces on mesenchymal stem cell (MSC) differentiation were examined in a fundamental study of the concept of environmental cell sensing. The study showed that differentiation of MSCs is directed by the stiffness of the culture matrix. On soft collagen gels that mimic the elasticity of brain tissue $(0.1-1 \mathrm{kPa})$, MSCs tend to adhere, spread, and exhibit a neurogenic phenotype. MSCs cultured on tenfold stiffer matrices that mimic muscle elasticity $(8-17 \mathrm{kPa})$ become spindle-shaped, similar to myoblasts. When cultured on matrices that mimic the stiffness of bone osteoid (25-40 kPa), the MSC phenotype becomes osteoblast-like with greater expression of osteogenic genes [88]. This work, along with similar studies, implies that a cell is able to sense its mechanical environment and that mechanical signaling itself can regulate the differentiation of MSCs into different tissues.

More recently, Padmaja Tummala et al. identified the presence of primary cilia on MSCs and determined their role in MSC differentiation. MSCs require primary cilia not only during their differentiation but also to maintain the

phenotypes of differentiated cells [89]. In addition, there is evidence that MSC differentiation into chondrocytes and osteocytes is regulated by mechanical signals [90]. Our research group is working on tissue regeneration to elucidate repair mechanisms, especially in OA (Fig. 1) and rheumatoid arthritis. OA is a chronic degenerative disease characterized by articular cartilage degeneration, and it is multifactorial in origin [89]. Primary cilia are present on chondrocytes, and the percentage of ciliated cells and the lengths of the cilia within OA tissue are higher compared to the normal tissue [65], although the implications of these facts have yet to be elucidated.

We have isolated CPCs from subjects in late-stage OA and characterized their role in the repair of diseased articular cartilage. CPCs have tremendous chondrogenic and regenerative potential. These cells are positive for stem cell markers and exhibit stem cell properties such as clonogenicity, multipotency, and migratory activity. Recently, we identified primary cilia projecting from the surfaces of CPCs using antibodies against acetylated alpha tubulin. Our laboratory is focused on using mechanobiological approaches to investigate the role of primary cilia in differentiation of CPCs into chondrocytes [91, 92]. 


\section{Conclusions and perspectives}

Owing to the involvement of primary cilia in fundamental cellular processes, mutations in primary ciliary proteins result in diverse diseases such as cystic kidney diseases, obesity, and retinal degeneration. Recent studies have presented a comprehensive concept that primary cilia are acting as dual sensors for physical and chemical cues. Therefore, over the past few years, many researchers have been paying attention to primary cilia to understand their role in development and diseases. Here, we have reviewed the basic role of primary cilia in mechanotransduction and their possible impact on cartilaginous tissues. Additionally, our results show that primary cilia project not only from the surface of human osteoarthritic chondrocytes but also from the surface of chondrogenic progenitor cells. One future line of research should be to elucidate the role of the primary cilia in chondrogenic differentiation to enhance the potential of cartilage repair.

Acknowledgments We would like to thank the American Journal Experts for editing the English language.

Open Access This article is distributed under the terms of the Creative Commons Attribution Noncommercial License which permits any noncommercial use, distribution, and reproduction in any medium, provided the original author(s) and source are credited.

\section{References}

1. Carter DR, Mikic B, Padian K (1998) Epigenetic mechanical factors in the evolution of long bone epiphyses. Zool J Linn Soc 123:163-178

2. Carter DR, Beaupré GS (2001) Skeletal function and form: mechanobiology of skeletal development, aging, and regeneration. Cambridge University Press, New York, p 332

3. Mikic B, Johnson TL, Chhabra AB, Schalet BJ, Wong M, Hunziker EB (2000) Differential effects of embryonic immobilization on the development of fibrocartilaginous skeletal elements. J Rehabil Res Dev 37:127-133

4. Osborne AC, Lamb KJ, Lewthwaite JC, Dowthwaite GP, Pitsillides AA (2002) Short-term rigid and flaccid paralyses diminish growth of embryonic chick limbs and abrogate joint cavity formation but differentially preserve pre-cavitated joints. J Musculoskelet Neuronal Interact 2:448-456

5. Murray PD, Drachman DB (1969) The role of movement in the development of joints and related structures: the head and neck in the chick embryo. J Embryol Exp Morphol 22:349-371

6. Hall BK, Herring SW (1990) Paralysis and growth of the musculoskeletal system in the embryonic chick. J Morphol 206:45-56

7. Hosseini A, Hogg DA (1991) The effects of paralysis on skeletal development in the chick embryo. I. General effects. J Anat 177:159-168

8. Hosseini A, Hogg DA (1991) The effects of paralysis on skeletal development in the chick embryo. II. Effects on histogenesis of the tibia. J Anat 177:169-178

9. Wong M, Germiller J, Bonadio J, Goldstein SA (1993) Neuromuscular atrophy alters collagen gene expression, pattern formation, and mechanical integrity of the chick embryo long bone. Prog Clin Biol Res 383B:587-597

10. Resnick N, Collins T, Atkinson W, Bonthron DT, Dewey C F Jr, Gimbron M A Jr (1993) Platelet-derived growth factor B chain promoter contains a cis-acting fluid shear-stress-responsive element. Proc Natl Acad Sci USA 90:7908

11. Liu M, Liu J, Buch S, Tanswell AK, Post M (1995) Antisense oligonucleotides for PDGF-B and its receptor inhibit mechanical strain-induced fetal lung cell growth. Am J Physiol 269:L178L184

12. Reusch P, Wagdy H, Reusch R, Wilson E, Ives HE (1996) Mechanical strain increases smooth muscle and decreases nonmuscle myosin expression in rat vascular smooth muscle cells. Circ Res 79:1046-1053

13. Robbins JR, Evanko SP, Vogel KG (1997) Mechanical loading and TGF-beta regulate proteoglycan synthesis in tendon. Arch Biochem Biophys 342:203-211

14. Cillo J E Jr, Gassner R, Koepsel RR, Buckley MJ (2000) Growth factor and cytokine gene expression in mechanically strained human osteoblast-like cells: implications for distraction osteogenesis. Oral Surg Oral Med Oral Pathol Oral Radiol Endod 90:147-154

15. Wu Q, Zhang Y, Chen Q (2001) Indian hedgehog is an essential component of mechanotransduction complex to stimulate chondrocyte proliferation. J Biol Chem 276:35290-35296

16. Zheng W, Seftor EA, Meininger CJ, Hendrix MJ, Tomanek RJ (2001) Mechanisms of coronary angiogenesis in response to stretch: role of VEGF and TGF-beta. Am J Physiol Heart Circ Physiol 280:H909-H917

17. Lindahl GE, Chambers RC, Papakrivopoulou J, Dawson SJ, Jacobsen MC, Bishop JE, Laurent GJ (2002) Activation of fibroblast procollagen alpha 1(I) transcription by mechanical strain is transforming growth factor-beta-dependent and involves increased binding of CCAAT-binding factor (CBF/NF-Y) at the proximal promoter. J Biol Chem 277:6153-6161

18. Liu M, Qin Y, Liu J, Tanswell AK, Post M (1996) Mechanical strain induces pp60src activation and translocation to cytoskeleton in fetal rat lung cells. J Biol Chem 271:7066-7071

19. Han B, Bai XH, Lodyga M, Xu J, Yang BB, Keshavjee S, Post M, Liu M (2004) Conversion of mechanical force into biochemical signaling. J Biol Chem 279:54793-54801

20. Jiang G, Huang AH, Cai Y, Tanase M, Sheetz MP (2006) Rigidity sensing at the leading edge through alphavbeta3 integrins and RPTPalpha. Biophys J 90:1804-1809

21. Wang H, Riha GM, Yan S, Li M, Chai H, Yang H, Yao Q, Chen C (2005) Shear stress induces endothelial differentiation from a murine embryonic mesenchymal progenitor cell line. Arterioscler Thromb Vasc Biol 25:1817-1823

22. Na S, Collin O, Chowdhury F, Tay B, Ouyang M, Wang Y, Wang N (2008) Rapid signal transduction in living cells is a unique feature of mechanotransduction. Proc Natl Acad Sci USA 105:6626-6631

23. Li S, Kim M, Hu YL, Jalali S, Schlaepfer DD, Hunter T, Chien S, Shyy JY (1997) Fluid shear stress activation of focal adhesion kinase. Linking to mitogen-activated protein kinases. J Biol Chem 272:30455-30462

24. Smith PG, Garcia R, Kogerman L (1998) Mechanical strain increases protein tyrosine phosphorylation in airway smooth muscle cells. Exp Cell Res 239:353-360

25. Leucht P, Kim JB, Currey JA, Brunski J, Helms JA (2007) FAKmediated mechanotransduction in skeletal regeneration. PLoS One 2:e390

26. Yamazaki T, Komuro I, Kudoh S, Zou Y, Shiojima I, Mizuno T, Takano H, Hiroi Y, Ueki K, Tobe K (1995) Mechanical stress activates protein kinase cascade of phosphorylation in neonatal rat cardiac myocytes. J Clin Invest 96:438-446 
27. Takahashi M, Berk BC (1996) Mitogen-activated protein kinase (ERK1/2) activation by shear stress and adhesion in endothelial cells. Essential role for a herbimycin-sensitive kinase. J Clin Invest 98:2623-2631

28. Jalali S, Li YS, Sotoudeh M, Yuan S, Li S, Chien S, Shyy JY (1998) Shear stress activates p60src-Ras-MAPK signaling pathways in vascular endothelial cells. Arterioscler Thromb Vasc Biol 18:227-234

29. MacKenna DA, Dolfi F, Vuori K, Ruoslahti E (1998) Extracellular signal-regulated kinase and c-Jun NH2-terminal kinase activation by mechanical stretch is integrin-dependent and matrix-specific in rat cardiac fibroblasts. $J$ Clin Invest 101:301-310

30. Schmidt C, Pommerenke H, Durr F, Nebe B, Rychly J (1998) Mechanical stressing of integrin receptors induces enhanced tyrosine phosphorylation of cytoskeletally anchored proteins. J Biol Chem 273:5081-5085

31. Chen NX, Ryder KD, Pavalko FM, Turner CH, Burr DB, Qiu J, Duncan RL (2000) $\mathrm{Ca}(2+)$ regulates fluid shear-induced cytoskeletal reorganization and gene expression in osteoblasts. Am J Physiol Cell Physiol 278:C989-C997

32. Iqbal J, Zaidi M (2005) Molecular regulation of mechanotransduction. Biochem Biophys Res Commun 328:751-755

33. Sadoshima J, Izumo S (1997) The cellular and molecular response of cardiac myocytes to mechanical stress. Annu Rev Physiol 59:551-571

34. Liu M, Tanswell AK, Post M (1999) Mechanical force-induced signal transduction in lung cells. Am J Physiol 277:L667-L683

35. Wang JH, Li B (2010) Mechanics rules cell biology. Sports Med Arthrosc Rehabil Ther Technol 2:16

36. Singla V, Reiter JF (2006) The primary cilium as the cell's antenna: signaling at a sensory organelle. Science 313:629-633

37. Wilsman NJ (1978) Cilia of adult canine articular chondrocytes. J Ultrastruct Res 64:270-281

38. Wilsman NJ, Farnum CE, Reed-Aksamit DK (1980) Incidence and morphology of equine and murine chondrocytic cilia. Anat Rec 197:355-361

39. Poole CA, Jensen CG, Snyder JA, Gray CG, Hermanutz VL, Wheatley DN (1997) Confocal analysis of primary cilia structure and colocalization with the Golgi apparatus in chondrocytes and aortic smooth muscle cells. Cell Biol Int 21:483-494

40. Poole CA, Zhang ZJ, Ross JM (2001) The differential distribution of acetylated and detyrosinated alpha-tubulin in the microtubular cytoskeleton and primary cilia of hyaline cartilage chondrocytes. J Anat 199:393-405

41. Jensen CG, Poole CA, McGlashan SR, Marko M, Issa ZI, Vujcich $\mathrm{KV}$, Bowser SS (2004) Ultrastructural, tomographic and confocal imaging of the chondrocyte primary cilium in situ. Cell Biol Int 28:101-110

42. McGlashan SR, Jensen CG, Poole CA (2006) Localization of extracellular matrix receptors on the chondrocyte primary cilium. J Histochem Cytochem 54:1005-1014

43. Zhang Q, Murcia NS, Chittenden LR, Richards WG, Michaud EJ, Woychik RP, Yoder BK (2003) Loss of the Tg737 protein results in skeletal patterning defects. Dev Dyn 227:78-90

44. Gouttenoire J, Valcourt U, Bougault C, Aubert-Foucher E, Arnaud E, Giraud L, Mallein-Gerin F (2007) Knockdown of the intraflagellar transport protein IFT46 stimulates selective gene expression in mouse chondrocytes and affects early development in zebrafish. J Biol Chem 282:30960-30973

45. Haycraft CJ, Zhang Q, Song B, Jackson WS, Detloff PJ, Serra R, Yoder BK (2007) Intraflagellar transport is essential for endochondral bone formation. Development 134:307-316

46. Koyama E, Young B, Nagayama M, Shibukawa Y, EnomotoIwamoto M, Iwamoto M, Maeda Y, Lanske B, Song B, Serra R, Pacifici M (2007) Conditional Kif3a ablation causes abnormal hedgehog signaling topography, growth plate dysfunction, and excessive bone and cartilage formation during mouse skeletogenesis. Development 134:2159-2169

47. McGlashan SR, Haycraft CJ, Jensen CG, Yoder BK, Poole CA (2007) Articular cartilage and growth plate defects are associated with chondrocyte cytoskeletal abnormalities in Tg737orpk mice lacking the primary cilia protein polaris. Matrix Biol 26:234-246

48. Ruiz-Perez VL, Blair HJ, Rodriguez-Andres ME, Blanco MJ, Wilson A, Liu YN, Miles C, Peters H, Goodship JA (2007) Evc is a positive mediator of Ihh-regulated bone growth that localises at the base of chondrocyte cilia. Development 134:2903-2912

49. Song JK, Niimi Y, Kupersmith MJ, Berenstein A (2007) Postnatal growth and development of a cerebral arteriovenous malformation on serial magnetic resonance imaging in a child with hemangiomatosis. Case report. J Neurosurg 106:384-387

50. Salter DM, Robb JE, Wright MO (1997) Electrophysiological responses of human bone cells to mechanical stimulation: evidence for specific integrin function in mechanotransduction. J Bone Miner Res 12:1133-1141

51. Hara F, Fukuda K, Asada S, Matsukawa M, Hamanishi C (2001) Cyclic tensile stretch inhibition of nitric oxide release from osteoblast-like cells is both $\mathrm{G}$ protein and actin-dependent. J Orthop Res 19:126-131

52. Hung CT, Allen FD, Pollack SR, Brighton CT (1996) Intracellular $\mathrm{Ca}^{2+}$ stores and extracellular $\mathrm{Ca}^{2+}$ are required in the realtime $\mathrm{Ca}^{2+}$ response of bone cells experiencing fluid flow. J Biomech 29:1411-1417

53. Malone AM, Anderson CT, Tummala P, Kwon RY, Johnston TR, Stearns T, Jacobs CR (2007) Primary cilia mediate mechanosensing in bone cells by a calcium-independent mechanism. Proc Natl Acad Sci USA 104:13325-13330

54. Danciu TE, Adam RM, Naruse K, Freeman MR, Hauschka PV (2003) Calcium regulates the PI3K-Akt pathway in stretched osteoblasts. FEBS Lett 536:193-197

55. Kanno T, Takahashi T, Tsujisawa T, Ariyoshi W, Nishihara T (2007) Mechanical stress-mediated Runx2 activation is dependent on Ras/ERK1/2 MAPK signaling in osteoblasts. J Cell Biochem 101:1266-1277

56. Wadhwa S, Choudhary S, Voznesensky M, Epstein M, Raisz L, Pilbeam C (2002) Fluid flow induces COX-2 expression in MC3T3-E1 osteoblasts via a PKA signaling pathway. Biochem Biophys Res Commun 297:46-51

57. Ott CE, Bauer S, Manke T, Ahrens S, Rodelsperger C, Grunhagen J, Kornak U, Duda G, Mundlos S, Robinson PN (2009) Promiscuous and depolarization-induced immediate-early response genes are induced by mechanical strain of osteoblasts. J Bone Miner Res 24:1247-1262

58. Serra R (2007) Intraflagellar transport in skeletal development. J Musculoskelet Neuronal Interact 7:302-303

59. Kolpakova-Hart E, Jinnin M, Hou B, Fukai N, Olsen BR (2007) Kinesin-2 controls development and patterning of the vertebrate skeleton by Hedgehog- and Gli3-dependent mechanisms. Dev Biol 309:273-284

60. St-Jacques B, Hammerschmidt M, McMahon AP (1999) Indian hedgehog signaling regulates proliferation and differentiation of chondrocytes and is essential for bone formation. Genes Dev 13:2072-2086

61. Kinumatsu T, Shibukawa Y, Yasuda T, Nagayama M, Yamada S, Serra R, Pacifici M, Koyama E (2011) TMJ development and growth require primary cilia function. J Dent Res 90(8):988-994

62. Shao YY, Wang L, Welter FJ, Ballock TR (2011) Primary cilia modulate Ihh signal transduction in response to hydrostatic loading of growth plate chondrocytes. Bone 50:79-84

63. Poole CA, Flint MH, Beaumont BW (1985) Analysis of the morphology and function of primary cilia in connective tissues: a cellular cybernetic probe? Cell Motil 5:175-193 
64. Praetorius HA, Praetorius J, Nielsen S, Frokiaer J, Spring KR (2004) Beta1-integrins in the primary cilium of MDCK cells potentiate fibronectin-induced $\mathrm{Ca}^{2+}$ signaling. Am J Physiol Renal Physiol 287:F969-F978

65. McGlashan SR, Cluett EC, Jensen CG, Poole CA (2008) Primary cilia in osteoarthritic chondrocytes: from chondrons to clusters. Dev Dyn 237:2013-2020

66. McGlashan SR, Knight MM, Chowdhury TT, Joshi P, Jensen GG, Kennedy S, Poole AC (2010) Mechanical loading modulates chondrocyte primary cilia incidence and length. Cell Biol Int 34:441-446

67. Farnum CE, Wilsman NJ (2011) Orientation of primary cilia of articular chondrocytes in three dimensional space. Anat Rec (Hoboken) 294(3):533-549

68. Reich A, Jaffe N, Tong A, Lavelin I, Genina O, Pines M, Sklan D, Nussinovitch A, Monsonego-Ornan E (2005) Weight loading young chicks inhibits bone elongation and promotes growth plate ossification and vascularization. J Appl Physiol 98:2381-2389

69. Reich A, Sharir A, Zelzer E, Hacker L, Monsonego-Ornan E, Shahar R (2008) The effect of weight loading and subsequent release from loading on the postnatal skeleton. Bone 43:766-774

70. Reich A, Maziel SS, Ashkenazi Z, Ornan EM (2010) Involvement of matrix metalloproteinases in the growth plate response to physiological mechanical load. J Appl Physiol 108:172-180

71. Verghese E, Zhuang J, Saiti D, Ricardo SD, Deane JA (2011) In vitro investigation of renal epithelial injury suggests that primary cilium length is regulated by hypoxia-inducible mechanisms. Cell Biol Int 35:909-913

72. Inglis PN, Boroevich KA, Leroux MR (2006) Piecing together a ciliome. Trends Genet 22:491-500

73. Gherman A, Davis EE, Katsanis N (2006) The ciliary proteome database: an integrated community resource for the genetic and functional dissection of cilia. Nat Genet 38:961-962

74. Ostrowski LE, Blackburn K, Radde KM, Moyer MB, Schlatzer DM, Moseley A, Boucher RC (2002) A proteomic analysis of human cilia: identification of novel components. Mol Cell Proteomics $1: 451-465$

75. Rosenbaum JL, Witman GB (2002) Intraflagellar transport. Natl Rev Mol Cell Biol 3:813-825

76. Pedersen LB, Rosenbaum JL (2008) Intraflagellar transport (IFT) role in ciliary assembly, resorption and signalling. Curr Top Dev Biol 85:23-61

77. Praetorius HA, Spring KR (2005) A physiological view of the primary cilium. Annu Rev Physiol 67:515-529

78. Temiyasathit S, Jacobs CR (2010) Osteocyte primary cilium and its role in bone mechanotransduction. Ann N Y Acad Sci 1192:422-428
79. Lehman JM, Michaud EJ, Schoeb TR, Aydin-Son Y, Miller M, Yoder BK (2008) The Oak Ridge polycystic kidney mouse: modeling ciliopathies of mice and men. Dev Dyn 237:1960-1971

80. Quinlan RJ, Tobin JL, Beales PL (2008) Modeling ciliopathies: primary cilia in development and disease. Curr Top Dev Biol 84:249-310

81. Veland IR, Awan A, Pedersen LB, Yoder BK, Christensen ST (2009) Primary cilia and signaling pathways in mammalian development, health and disease. Nephron Physiol 111:p39-p53

82. Badano JL, Mitsuma N, Beales PL, Katsanis N (2006) The ciliopathies: an emerging class of human genetic disorders. Annu Rev Genomics Hum Genet 7:125-148

83. Huangfu D, Anderson KV (2006) Signaling from Smo to Ci/Gli: conservation and divergence of Hedgehog pathways from Drosophila to vertebrates. Development 133:3-14

84. Alvarez J, Sohn P, Zeng X, Doetschman T, Robbins DJ, Serra R (2002) TGFbeta2 mediates the effects of hedgehog on hypertrophic differentiation and PTHrP expression. Development 129:1913-1924

85. Kobayashi T, Soegiarto DW, Yang Y, Lanske B, Schipani E, McMahon AP, Kronenberg HM (2005) Indian hedgehog stimulates periarticular chondrocyte differentiation to regulate growth plate length independently of PTHrP. J Clin Invest 115:1734-1742

86. Kronenberg HM (2006) PTHrP and skeletal development. Ann N Y Acad Sci 1068:1-13

87. Rohatgi R, Milenkovic L, Scott MP (2007) Patched1 regulates hedgehog signaling at the primary cilium. Science 317:372-376

88. Engler AJ, Sen S, Sweeney HL, Discher DE (2006) Matrix elasticity directs stem cell lineage specification. Cell 126:677-689

89. Tummala P, Arnsdorf EJ, Jacobs CR (2010) The role of primary cilia in mesenchymal stem cell differentiation: a pivotal switch in guiding lineage commitment. Cell Mol Bioeng 3:207-212

90. Kelly DJ, Jacobs CR (2010) The role of mechanical signals in regulating chondrogenesis and osteogenesis of mesenchymal stem cells. Birth Defects Res C Embryo Today 90:75-85

91. Koelling S, Miosge N (2010) Sex differences of chondrogenic progenitor cells in late stages of osteoarthritis. Arthritis Rheum 62:1077-1087

92. Koelling S, Kruegel J, Irmer M, Path JR, Sadowski B, Miro X, Miosge N (2009) Migratory chondrogenic progenitor cells from repair tissue during the later stages of human osteoarthritis. Cell Stem Cell 4:324-335 


\section{Chapter 3}

A discoidin domain receptor 1 knockout mouse as a novel model for osteoarthritis of the temporomandibular joint

Boris Schminke ${ }^{1 *}$, Hayat Muhammad ${ }^{1 *}$, Christa Bode ${ }^{1}$, Boguslawa Sadowski ${ }^{1}$, Regina Gerter $^{1}$, Nikolaus Gersdorff', Ralf Bürgers ${ }^{1}$, Efrat Monsonego-Ornan², Vicki Rosen ${ }^{3 \#}$ and Nicolai Miosge ${ }^{1 \#}$

${ }^{1}$ Georg August University, Tissue Regeneration Work Group,

Department of Prosthodontics Goettingen, Germany

${ }^{2}$ Faculty of Agricultural, Food and Environmental Sciences,

Institute of Biochemistry and Nutrition, Hebrew University,

POB 12, 76100 Rehovot, Israel

${ }^{3}$ Developmental Biology, Harvard School of Dental Medicine,

188 Longwood Avenue, Boston, MA 02115, USA

\#Both are corresponding authors

"Both authors contributed equally to this work

Hayat Muhammad's contribution: FACS data analysis, immunocytochemistry, gene-silencing experiments, PCR, cell culture and partial manuscript preparation. 


\title{
A discoidin domain receptor 1 knock-out mouse as a novel model for osteoarthritis of the temporomandibular joint
}

\author{
Boris Schminke $\cdot$ Hayat Muhammad $\cdot$ Christa Bode $\cdot$ Boguslawa Sadowski $\cdot$ \\ Regina Gerter • Nikolaus Gersdorff • Ralf Bürgers • Efrat Monsonego-Ornan • \\ Vicki Rosen • Nicolai Miosge
}

Received: 12 February 2013 / Revised: 19 July 2013 / Accepted: 22 July 2013

(C) Springer Basel 2013

\begin{abstract}
Discoidin domain receptor 1 (DDR-1)-deficient mice exhibited a high incidence of osteoarthritis (OA) in the temporomandibular joint (TMJ) as early as 9 weeks of age. They showed typical histological signs of OA, including surface fissures, loss of proteoglycans, chondrocyte cluster formation, collagen type I upregulation, and atypical collagen fibril arrangements. Chondrocytes isolated from the TMJs of DDR-1-deficient mice maintained their osteoarthritic characteristics when placed in culture. They expressed high levels of runx-2 and collagen type I, as well as low levels of sox-9 and aggrecan. The expression of DDR-2, a key factor in OA, was increased. DDR1-deficient chondrocytes from the TMJ were positively influenced towards chondrogenesis by a three-dimensional
\end{abstract}

B. Schminke and H. Muhammad contributed equally to this work.

Electronic supplementary material The online version of this article (doi:10.1007/s00018-013-1436-8) contains supplementary material, which is available to authorized users.

B. Schminke $\cdot$ H. Muhammad · C. Bode $\cdot$ B. Sadowski ·

R. Gerter $\cdot$ N. Gersdorff $\cdot$ R. Bürgers $\cdot$ N. Miosge $(\bowtie)$

Oral Biology and Tissue Regeneration Work Group, Department of Prosthodontics, Medical Faculty, Georg-August-University,

Robert Koch Straße 40, 37075 Goettingen, Germany

e-mail: nmiosge@gwdg.de

E. Monsonego-Ornan

Robert H. Smith Faculty of Agriculture, Food and Environment, Institute of Biochemistry, Food Science and Nutrition,

The Hebrew University of Jerusalem, P.O. Box 12,

76100 Rehovot, Israel

\section{Rosen $(\square)$}

Developmental Biology, Harvard School of Dental Medicine, 188 Longwood Avenue, Boston, MA 02115, USA

e-mail: vicki_rosen@hsdm.harvard.edu matrix combined with a runx-2 knockdown or stimulation with extracellular matrix components, such as nidogen-2. Therefore, the DDR-1 knock-out mouse can serve as a novel model for temporomandibular disorders, such as OA of the TMJ, and will help to develop new treatment options, particularly those involving tissue regeneration.

Keywords Temporomandibular joint · Osteoarthritis . Extracellular matrix $\cdot$ Collagen receptor $\cdot$ Chondrocyte signaling

\section{Introduction}

Temporomandibular disorders (TMDs) are structural, functional, biochemical, and physiological dysregulations of the muscle or the temporomandibular joint (TMJ). It is estimated that $10-40 \%$ of the population between 18 and 45 years of age present symptoms or signs of TMD, and nearly $10 \%$ are classified as suffering from osteoarthritis $(\mathrm{OA})$ in this joint $[1,2]$. Because OA of the TMJ is usually diagnosed only in the later stages of the disease, it is likely that the true incidence of OA of the TMJ may be higher [1]. TMD patients experience severe pain in the mastication muscles, joint clicking, displacement or perforation of the articular disc, and inflammatory or degenerative changes in the joint itself. Untreated TMDs ultimately result in OA of the TMJ [2]. The current therapeutic interventions for OA primarily provide short-term symptomatic relief, and almost all patients ultimately require joint replacement [3]. While the pathogenesis of OA of the TMJ has some special features, the general aspects are similar to those of OA in other joints in which the imbalance between cartilage degradation and matrix synthesis ultimately results in the complete loss of joint function [4]. Within the joint, the articular cartilage 
is responsible for the smooth transmission of force from one bone to another, thereby allowing painless skeletal movements [5]. The maintenance of articular cartilage extracellular matrix $(\mathrm{ECM})$ is required for biomechanical functions, such as rigidity and resistance to compression and shear forces $[6,7]$. Chondrocytes, the cells responsible for cartilage tissue homeostasis, are embedded in a framework of collagens that, together with proteoglycans and glycoproteins [8], act as linking proteins to stabilize the collagen network. The chondrocytes in the articular cartilage do not make direct cell-to-cell contact; instead, they rely on cellmatrix interactions [9] via integrins [10] or DDRs [11] for communication. Cartilage appears to have a low capacity for regeneration, and ECM degradation overrides the wellknown tissue regeneration attempts. Recently, we identified chondrogenic progenitor cells (CPCs), which drive these regeneration processes. During the late stages of OA, CPCs are located in the repair tissue of human articular knee cartilage. They exhibit stem cell characteristics such as clonogenicity, multipotency, and migratory activity and exhibit a high chondrogenic potential $[12,13]$. Whether CPCs might also play a role in the regeneration attempts of the TMJ is unknown, although this information is of importance in designing new therapies to treat TMD. Because human TMJ specimens are sparse, we aimed to establish a novel mouse model for OA of the TMJ. Histological analyses of the existing mouse models currently used to study OA, including ICR mice [14], Del 1 mice [15], Cho mice [16], mechanically-induced OA via partial discectomy [17], and Col IX knock-out (KO) mice [18], showed a low incidence of TMJ OA. In contrast, we demonstrate that Discoidin domain receptor 1 (DDR-1)-deficient mice exhibit a high incidence of TMJ OA beginning at an early age, and are suitable as a model for TMD. Receptor tyrosine kinases such as DDRs are widely expressed in human and mouse tissues. The binding of collagen to DDR-1 results in tyrosine kinase activation [19]. This activation causes downstream signaling via Shc [20] or FRS2 [21], altering the gene expression levels of ECM molecules [19] that are important for maintaining healthy articular cartilage. Here, we demonstrate that DDR1-deficient mice display the histological characteristics typical of OA of the TMJ. Furthermore, isolated osteoarthritic DDR-1 KO TMJ cells can be converted to chondrocytes with a more normal phenotype, rendering cell biological interventions possible.

\section{Materials and methods}

Tissue sources and preparation

Animals were obtained according to the regulations of the Animal Welfare Act of the County of Lower Saxony,
Table 1 Grading of TMJ cartilage destruction in KO mice

\begin{tabular}{llll}
\hline $\begin{array}{l}\text { Age at } \\
\text { examination } \\
\text { (weeks) }\end{array}$ & $\begin{array}{l}\text { Number of } \\
\text { sacrificed KO } \\
\text { mice }\end{array}$ & $\begin{array}{l}\text { Average } \\
\text { modified Mankin } \\
\text { score (0-23) }\end{array}$ & $\begin{array}{l}\text { Average of } \\
\text { OA prevalence } \\
\text { (in \%) }\end{array}$ \\
\hline 6 & 8 & 1 & 50 \\
9 & 21 & 15 & 83 \\
12 & 28 & 17 & 89 \\
24 & 17 & 18 & 91 \\
28 & 12 & 18 & 91 \\
\hline
\end{tabular}

Table 2 Grading of TMJ cartilage destruction in WT mice

\begin{tabular}{llll}
\hline $\begin{array}{l}\text { Age at } \\
\text { examination } \\
\text { (weeks) }\end{array}$ & $\begin{array}{l}\text { Number of } \\
\text { sacrificed WT } \\
\text { mice }\end{array}$ & $\begin{array}{l}\text { Average modi- } \\
\text { fied } \\
\text { Mankin score } \\
(0-23)\end{array}$ & $\begin{array}{l}\text { Average of } \\
\text { OA prevalence } \\
\text { (in \%) }\end{array}$ \\
\hline 6 & 6 & 0 & 0 \\
9 & 15 & 0 & 0 \\
12 & 20 & 0 & 0 \\
24 & 20 & 1 & 15 \\
28 & 15 & 2 & 20 \\
\hline
\end{tabular}

Germany. The generation and genotyping of DDR-1 null mice has been described previously [22]. Cartilage destruction was graded according to the modified Mankin score [23]; however, toluidine blue staining was used instead of safranin-O staining. Accordingly, healthy cartilage received a minimum score of zero points, and late stage OA received a maximum score of 23 points. Three histologists evaluated the tissues independently. The scores of $\mathrm{KO}$ and wild-type (WT) mice are shown in Tables 1 and 2.

\section{Micro-computed tomography}

Three DDR-1 knockout and three wild-type (WT) mice were sacrificed at ages ranging from 9 to 12 weeks, and their jaws were prepared. The mineral content was measured in the subchondral bone of the articular surface of the mandible from each group using $\mu-\mathrm{CT}$ scanning. The bones were scanned with a GE eXplore Locus SP Pre-Clinical Specimen MicroCT instrument (GE Medical Systems, Muenchen, Germany) operated at a 13-m isotropic voxel resolution. The specimens were immersed in water, and hydroxyapatite $\left(1.13 \mathrm{~g} / \mathrm{cm}^{3}\right)$ was included in each scan to provide reference values.

Antibodies for immunohistochemistry, immunocytochemistry, immunoblotting and FACS analysis

A monoclonal rat-anti-nidogen-1 antibody (JF4) and a polyclonal rabbit-anti-nidogen-2 antibody $(1080+E 2)$ 
were generously donated by Dr. T. Sasaki (University of Erlangen, Germany). The nidogen-1 and nidogen-2 antibodies have previously been demonstrated not to crossreact [8]. The anti-COMP antibody is an affinity purified polyclonal rabbit-anti-bovine antibody [24]. Goat-antirabbit or goat-anti-rat (Dako, Hamburg, Germany) secondary antibodies were used for immunostaining for light microscopy. DDR-1 (h-126, sc-8988), DDR-2 (h-108, sc-8989), aggrecan (4F4: sc-33695), runx-2 (M-70: sc-10758) and sox-9 (H-90: sc-2095) antibodies were obtained from Santa Cruz Biotechnology (Santa Cruz, CA, USA). Monoclonal anti-type I collagen (M-38) and an anti-type II collagen (CIIC1) antibodies were obtained from the Developmental Studies Hybridoma Bank, University of Iowa, USA. Intracellular FACS analysis was conducted using the Fix \& Perm kit ${ }^{\circledR}$ (Invitrogen, Darmstadt, Germany). The MMP-13 antibody (ab39012) was obtained from Abcam (Cambridge, MA, USA). Goatanti-mouse, goat-anti-rabbit-FITC (Dianova, Hamburg, Germany) and anti-mouse-PE/FITC monoclonal immunoglobulin isotype controls (BD Pharmingen, Mountain View, CA, USA) were used as secondary antibodies. For immunoblotting, we used a goat-anti-mouse antibody coupled with alkaline phosphatase and a pan- $\beta$-actin (Dako, Hamburg, Germany) or $\alpha$-tubulin (mouse monoclonal, DM1A; Sigma-Aldrich, St. Gallen, Switzerland) antibody as gel loading control. The detection of primary cilia was performed with anti-acetylated $\alpha$-tubulin antibody [6-11B-1] (ab24610), which was purchased from Abcam.

Antibody immunoreactions were also performed in the absence of primary antibody, as a negative control, and images show representatives of three individual experiments.

Light microscopic immunohistochemistry

Immunoperoxidase staining was performed on paraffinembedded tissue sections as follows. The tissues were deparaffinised, rehydrated, and rinsed for $10 \mathrm{~min}$ in PBS. Endogenous peroxidase was blocked by a 45 -min treatment with a solution of methanol and $3 \% \mathrm{H}_{2} \mathrm{O}_{2}$ in the dark. Each of the reactions was followed by rinsing for $10 \mathrm{~min}$ in PBS. The sections were pre-treated for $5 \mathrm{~min}$ with $10 \mu \mathrm{g} / \mathrm{ml}$ protease XXIV (P8038; Sigma, Deisenhofen, Germany) and chondroitinase (C3667-5UN; Sigma). The antibodies were applied at a dilution of 1:100 in PBS for $12 \mathrm{~h}$ at room temperature. A standard peroxidase-anti-peroxidase procedure followed with the application of a peroxidase-coupled goat-anti-rabbit antibody (Dako;) at a dilution of 1:150 in PBS for $1 \mathrm{~h}$ at room temperature. The color reaction was carried out with a DAB (diaminobenzidine) substrate [25].
Electron microscopy

For ultrastructural investigations, $1-\mathrm{mm}^{3}$ cartilage samples from the condyle were resected. All tissue samples were then fixed and embedded in Epon ${ }^{\circledR}$ (Serva, Heidelberg, Germany). Subsequently, semi-thin $(1 \mu \mathrm{m})$ and ultra-thin sections $(80 \mathrm{~nm})$ were cut. The ultra-thin sections were collected on Formvar ${ }^{\circledR}$-coated grids and stained as described elsewhere [26].

\section{Cell isolation and culture}

Standard explant cultures were established from 1-mm tissue specimens taken from the TMJ cartilage tissue of 9-week-old DDR-1 KO mice and their WT littermates. Care was taken to ensure that no bone tissue was included. After 10 days, outgrown chondrocytes were harvested, and $10^{3}$ cells $/ \mathrm{cm}^{2}$ were transferred to cell culture in Dulbecco's modified Eagle's medium (DMEM) supplemented with $10 \%$ fetal bovine serum (Invitrogen; Lot. nr. 41F2061K), gentamycin $(50 \mu \mathrm{g} / \mathrm{ml})$ and L-glutamine $(10 \mathrm{mM})$. Furthermore, we cultured TMJ chondrocytes for 3 weeks in alginate beads, which provides the three-dimensional environment that is important for the phenotypic stability of the chondrocytes [27]. To test the influence of the ECM components, 40,000 cells at $\mathrm{P} 2$ were mixed with a $1.2 \%$ alginate solution supplemented with either $125 \mathrm{ng} / \mathrm{ml}$ of laminin-1 (Dianova) or nidogen-2 (a kind gift from the late R. Timpl) or with $5 \mathrm{ng} / \mu 1$ of BMP-2 and BMP-4 and grown for 3 weeks in 3D in 6-well plates.

Immunofluorescence microscopy

The primary cells were transferred at P1 in 96-well plates for $16 \mathrm{~h}$, they were fixed with $70 \%$ ethanol and then incubated with $100 \mu$ l of primary antibody diluted 1:50 in PBS for $1 \mathrm{~h}$ at RT in the dark. When necessary, this step was followed by incubation with a secondary fluorescenceconjugated antibody (diluted 1:500) for $20 \mathrm{~min}$ at RT. Two washes with PBS were performed, followed by DAPI staining. The cells were examined under a fluorescence microscope, and the images were captured with a Nikon D90 camera (Düsseldorf, Germany).

\section{Confocal microscopy}

Cells were imaged with a FluoView1000 (Olympus) confocal microscope using a $\times 60$ NA1.35 UPLS-APO objective. The excitation/emission wavelengths for DAPI and TRITC were 405/425-520 nm and 561 nm/570-670 nm, respectively. Images were acquired at a resolution of $1,024 \times 1,024$ with the confocal pinhole set at airy disk 1 . Two sequential frames were acquired and averaged. 


\section{FACS analysis}

The cultured cells were suspended in PBS with the fluorescence-coupled antibodies listed above $(1 \mu 1$ added to $100 \mu 1$ containing $10^{6}$ cells) at RT for $1 \mathrm{~h}$ in the dark. Two subsequent washing steps were performed (with centrifugation for $10 \mathrm{~min}$ at $800 \mathrm{rpm}$ ). The cells were analyzed on a FACScan instrument (Becton-Dickinson, Mountain View, CA, USA) as described in detail elsewhere [28]. At least 10,000 living cells were analyzed. The data were evaluated with the aid of WinMDI v.2.9. For cell selection, we applied FACS Vantage SE (Becton-Dickinson). We performed analyses using the Cell Quest Pro 2000 software package.

Immunoblotting

Proteins were extracted using $5 \mathrm{M}$ guanidine hydrochloride and protease inhibitors, precipitated in ethanol, washed in PBS, precipitated again, and finally dissolved in PBS containing $0.4 \%$ SDS. SDS-PAGE was performed with a $6 \%$ acrylamide stacking gel and $12 \%$ separation gel. Gel loading was evaluated using $\alpha$-actin staining. The proteins were blotted onto nitrocellulose membranes, which were subsequently washed, and blocked, and immunoreactions were performed by exposure to antibodies diluted 1:500 in PBS. The secondary goat-anti-mouse antibody was diluted 1:1,000 and incubated with the membrane for $1 \mathrm{~h}$ at RT. Visualization was achieved by applying the ECL Prime Detection Regent (GE Healthcare, Muenchen, Germany), and certain results were quantified using the Image $\mathbf{J}^{\circledR}$ program.

\section{siRNA transfections}

For silencing experiments, we used the iLenti-GFP siRNA expression vector (Biocat, Heidelberg, Germany) in which the target sequence of the runx-2 siRNA (CAGCACGCTATTAAATCCAAATT) is under the control of the H1 and U6 promoters. For transfection confirmation and efficiency, the reporter gene GFP was placed under the CMV promoter. All control experiments were performed with the vector without the runx-2 silencing insert. Nucleofection of TMJ chondrocytes was performed according to the manufacturer's instructions (Lonza, Basel, Switzerland). Briefly, the TMJ chondrocytes were trypsinized and counted. The cells were centrifuged at 1,200 rpm for $10 \mathrm{~min}$, and $5 \times 10^{5}$ cells were resuspended in $100 \mu \mathrm{l}$ of the nucleofection reagent containing $2 \mu \mathrm{g}$ of plasmid DNA. To increase the transfection efficiency, the U-23 program was selected. Immediately after nucleofection, the TMJ chondrocytes were plated in warm culture medium in a T25 culture flask. The culture medium was replaced the next day to remove dead cells. The cells were harvested after $24 \mathrm{~h}$ after medium replacement.

\section{Overexpression}

Runx-2 was cloned into the pPM-C-His vector (ABM, Richmond, Canada) using standard procedures. The vector with the insert and the vector without the insert were transfected (PolyFect; Qiagen, Hilden, Germany) into the cells as described above.

RNA extraction and complementary DNA (cDNA) synthesis

Cells in P1 were directly lysed in RLT buffer, and the RNA was isolated according to the manufacturer's instructions (RNeasy Mini Kit; Qiagen, Chatsworth, CA, USA). RNA was reverse-transcribed into cDNA with the help of the Qiagen QuantiTect Reverse Transcription Kit, as per the manufacturer's instructions.

\section{Microarray analysis}

Quality control and the quantification of total RNA samples was performed prior to the microarray experiments (Agilent 2100 Bioanalyzer; Agilent Technologies, Palo Alto, CA, USA). We used equal amounts of total RNA from the condyles of each of three pairs of DDR-1 KO mice and two samples of their WT littermates. The microarray analysis was conducted at the university transcriptome facility using an Affymetrix whole-mouse genome chip (Affymetrix, Santa Clara, CA, USA). The microarray experiments were performed according to the manufacturer's protocols. A complete list of the genes present on the chip can be found at http://www.affymetrix.com/analysis/index.affx. The data were analyzed using Affymetrix Microarray Suite 5.0. Gene expression was evaluated using the Affymetrix Data Mining Tool 3.0. The entire dataset is published in a MIAME-compliant format in the GEO database with the accession number GSE35297 (http://www.ncbi.nlm.nih. gov/geo/).

\section{Quantitative rt RT-PCR}

PCR was performed in a final volume of $10 \mu$ l containing $5 \mu l$ Platinum SYBR Green qPCR SuperMixTM (Invitrogen), 20 pmol of each primer and $1 \mathrm{ng}$ of cDNA were added to a final volume of 10 . The primers were designed using Primer $3^{\circledR}$ software (http://frodo.wi.mit.edu/cgibin/ primer3/primer3). The primer sequences used are shown below: 
using SPSS software 13.0 (SPSS, Chicago, IL, USA). The results are reported as the mean values and standard deviations (SD). After testing for normal distribution and variance homogeneity, we performed a one-way analysis of variance (ANOVA) and a post hoc pairwise comparison of mean values. The Pearson correlation coefficients were calculated to examine the relationships between the parameters. A $p$ value $<0.05$ was considered significant.

\section{Results}

Structural and genomic aspects of OA of the TMJ in DDR-1 KO mice in vivo

Disordered cell matrix interactions play a central role in the development of OA [9]. Therefore, it is reasonable to hypothesize that collagen receptors, such as integrins [10] and DDRs, are involved in OA pathogenesis. Because severe $\mathrm{OA}$ is associated with bone structure alterations [30], we applied microcomputed tomography $(\mu \mathrm{CT})$ as a straightforward initial approach to examine DDR-1 KO TMJ. Three-dimensional reconstruction of the mandibular condyles of these mice showed a rugged subchondral bone surface and a flattening of the mandibular condyle (Fig. 1a, left, b), structural changes that are typical of TMD [2]. In contrast, the subchondral bone surface of WT mice was smooth and rounded (Fig. 1a, right, c), as expected for a normal joint. Furthermore, the DDR-1 KO mice exhibited a greater relative bone mineral density of the subchondral bone (Fig. 1d). Ultrastructurally, the joint surface of the mandibular condyle of 9-week-old DDR-1 KO mice demonstrated an altered collagen fiber network with loosely packed and randomly arranged collagen fibers in the cartilage (Fig. 1e, f). In comparison, the collagen fiber assembly in WT mice was parallel to the joint surface (Fig. 1g), and the arrangement appeared more compact (Fig. 1h). To investigate the overall changes in gene expression, we performed a microarray analysis of cartilage tissue samples from the mandibles of DDR-1-deficient and WT mice (Fig. 1i). Major changes in the DDR-1 KO mice were associated with ECM components. For example, the DDR-1 KO mice exhibited a lower expression of collagen type II, collagen type III, collagen type IX, aggrecan, and sox-9, while collagen type $\mathrm{X}$, nidogen-2, and runx-2 expression levels were increased; this expression pattern is typical of OA. At the histopathological level, toluidine blue staining (Fig. 1j vs. 1) indicated that, by 9 weeks of age, DDR-1 KO mice showed a decreased proteoglycan content at the joint surface of the mandibular condyle compared with their WT littermates. Moreover, loss of the superficial cartilage layer and deep surface fissures were observed in DDR-1 KO mice (Fig. 1k vs. m), but not in WT mice. These OA pendent experiments, and we statistically tested our results using separate specimens. The analyses were performed 

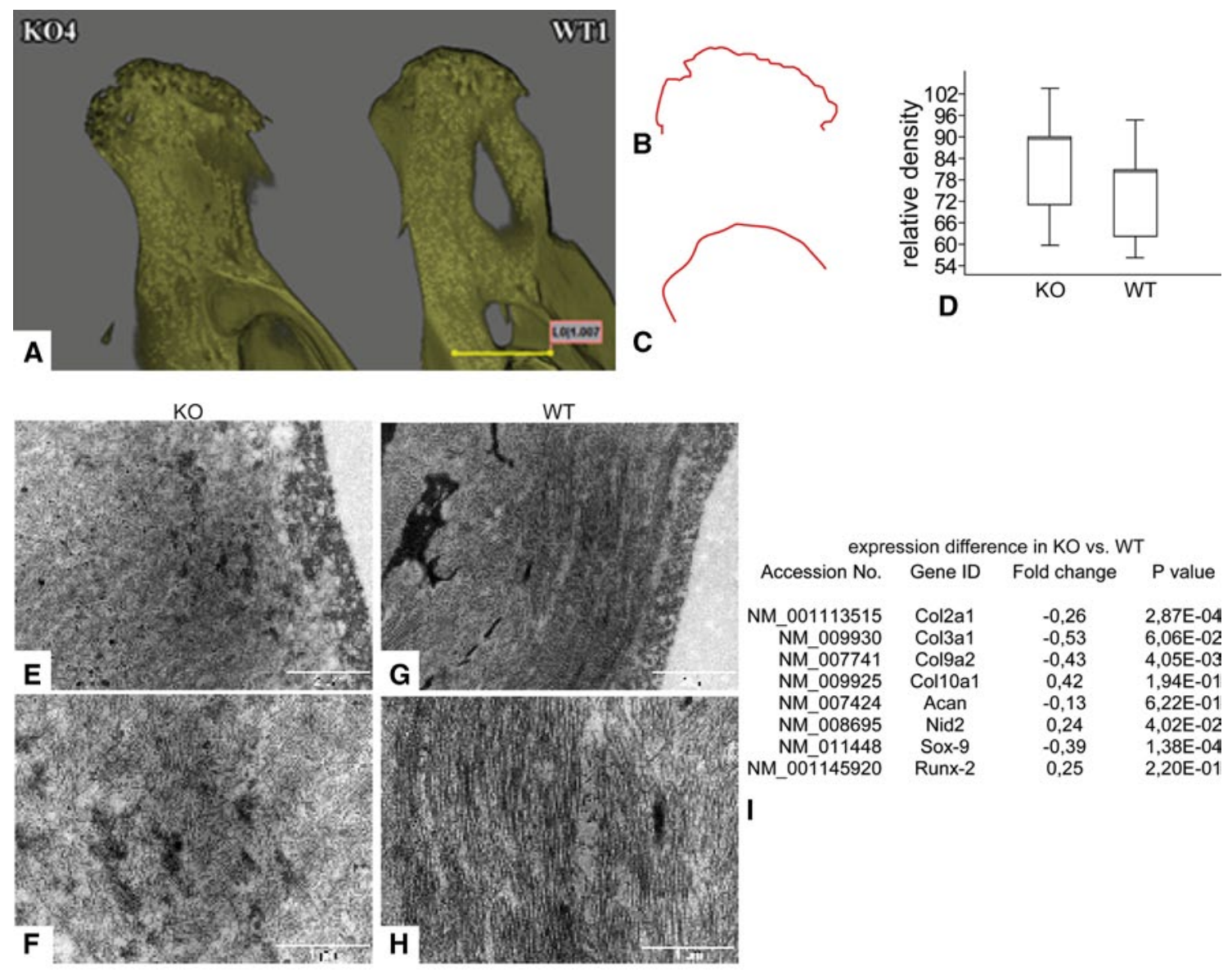

\begin{tabular}{rccc}
\multicolumn{5}{c}{ expression difference in KO vs. WT } \\
Accession No. & Gene ID & Fold change & P value \\
NM_001113515 & Col2a1 & $-0,26$ & $2,87 \mathrm{E}-04$ \\
NM_009930 & Col3a1 1 & $-0,53$ & $6,06 \mathrm{E}-02$ \\
NM_007741 & Col9a2 & $-0,43$ & $4,05 \mathrm{E}-03$ \\
NM_009925 & Col10a1 & 0,42 & $1,94 \mathrm{E}-01$ \\
NM_007424 & Acan & $-0,13$ & $6,22 \mathrm{E}-01$ \\
NM_008695 & Nid2 & 0,24 & $4,02 \mathrm{E}-02$ \\
NM_011448 & Sox-9 & $-0,39$ & $1,38 \mathrm{E}-04$ \\
NM_001145920 & Runx-2 & 0,25 & $2,20 \mathrm{E}-01$
\end{tabular}

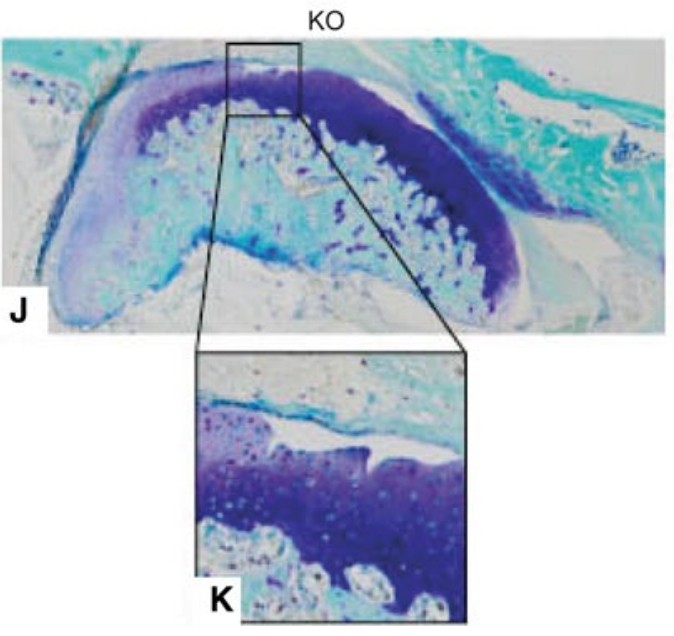

Fig. 1 Structural and genomic aspects of OA of the TMJ in DDR-1 KO mice in vivo. a The micro-CT 3D reconstructions of the condylus mandibulae of the DDR-1 KO mice (KO, always on the left side) and the WT (WT, always on the right side). b, c Outline of the subchondral bone surface. The KO mice (b) exhibited a rough surface and an abnormal bone structure compared to the WT (c). d The measurements of the condyle bone mineral density revealed that the KO mice had a higher bone density. e-h Ultrastructural analysis: e, $\mathbf{f}$ the collagen fiber arrangement was altered in the superficial layer of DDR-1 $\mathrm{KO}$ mice compared to the parallel fiber alignment observed in the WT

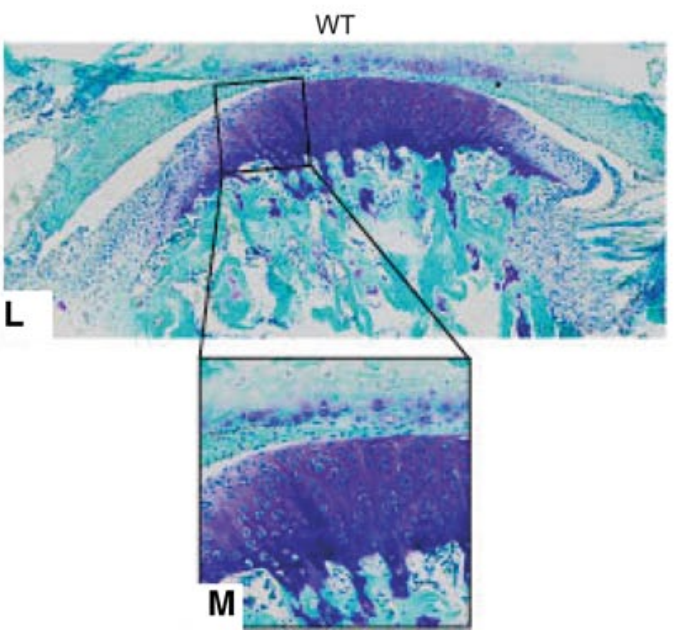

mice ( $\mathbf{g}, \mathbf{h})$. i A short list of the fold changes of OA-relevant genes found in the microarray analysis. The complete lists can be found at GEO, GSE35297. $\mathbf{j}-\mathbf{m}$ Toluidine blue histology of the condyles: $\mathbf{j}$, $\mathbf{k}$ the DDR-1 KO condyle exhibits well-known signs of OA such as reduced staining of the superficial zone, cluster formation and surface fissures; l, m normal WT condyles. Three pairs of DDR-1 KO mice condyles and samples from two of their WT littermates were used for microarray analysis. ( $n=6$, including $3 \mathrm{KO}$ mice and $3 \mathrm{WT}$ mice, for electron microscopy; $n=5$, including $3 \mathrm{KO}$ mice and $2 \mathrm{WT}$ mice for the microarray) 
features were also present in 24-week-old mice, as shown here for toluidine blue staining (Fig. 3i-1).

Molecular changes in the ECM of the TMJ during OA in vivo

Immunohistochemical analyses of the TMJs of 9-week-old DDR-1 KO mice revealed surface fissures, increased collagen type I, increased collagen fiber fibrillation, and an increased number of cells at the articular surface of the mandibular condyle (Fig. 2a vs. b). This area also exhibited collagen type I staining (Fig. $2 \mathrm{c}$ vs. d). Surface fissures also became visible in HE staining of 24-week-old KO mice (Fig. 3a, c) compared with the corresponding WT mice (Fig. 3b, d). These features have been well described and are specific for OA progression [30]. Collagen type I staining was also detected in 28-weekold KO mice (Fig. 3e vs. f). Furthermore, these KO mice also exhibit the typically destroyed joint surface (Fig. $3 g$ vs. h). The expression of collagen type II, the collagen typical of the hyaline cartilage, was decreased in the DDR-1-deficient mice compared with WT controls (Fig. 2e vs. f). This was especially apparent in the superficial layer of the articular cartilage of the TMJ of DDR-1 KO mice, from which collagen type II was absent (Fig. $2 \mathrm{~g}$ vs. h). The basement membrane proteins [31], most notably the nidogens, are involved as players in the pericellular matrix during the pathogenesis of human $\mathrm{OA}$ [6], and an increased amount of nidogen-2 is found primarily around elongated chondrocytes from the late stages of OA [8]. There were no differences in the localization of nidogen-1 in DDR-1 KO mice (Fig. 2i, k) compared with the WT mice (Fig. 2j, 1). A pericellular increase in nidogen-2 staining in the middle zone was observed in the TMJ of DDR-1-deficient mice (Fig. $2 \mathrm{~m}$ vs. n); however, less nidogen-2 staining was observed in the superficial and deeper layers (Fig. 2o vs. p). The same pattern was seen for collagen type IV in 24-weekold mice (Fig. $3 \mathrm{~m}-$ p). On the basis of these histopathological findings of $\mathrm{OA}$ in vivo, we isolated chondrocytes from the TMJs of DDR-1 KO mice for further in vitro studies.

\section{Cell isolation and characterization}

Mouse cartilage was separated from the subchondral bone under a stereomicroscope (Fig. 4a, b). After 10 days in culture, the cells from the articular cartilage isolated from DDR-1 KO mice migrated out of the tissue specimens (Fig. 4c). We found that DDR-1 KO TMJ chondrocytes exhibited low levels of sox-9 (a chondrogenic transcription factor; Fig. 4d) and aggrecan (Fig. 4g) mRNA but high levels of runx-2, an osteogenic transcription factor (Fig. 4e), and collagen type I (Fig. 4f) mRNA compared with cells from the WT mice. DDR-1 KO and WT TMJ chondrocytes were positive for runx-2 (Fig. 4h, k), collagen type I (Fig. 4i, l), and aggrecan (Fig. 4j, m) proteins. Chondrocytes from DDR-1 KO mice seemed to express higher levels of runx-2 (Fig. 4h) than WT chondrocytes (Fig. 4k), consistent with the results of our real-time RT-PCR experiments. After passage 6, the phenotype of the TMJ chondrocytes was altered. This dedifferentiation of chondrocytes in culture is a well-known phenomenon and is usually associated with higher passage numbers for cells in culture [32]. We noticed that DDR-1-deficient chondrocytes changed more obviously than WT cells and had smaller cell bodies and numerous cell protrusions compared with WT controls (data not shown). Therefore, we re-evaluated the protein expression levels of the DDR-1-deficient cells at passage 6. We found that both DDR-1 null cells and their WT counterparts were positive for the typical components of cartilage ECM, and both exhibited a chondrocytic nature (Fig. 4n). Even at passage 6, the DDR-1 KO cells and their WT counterparts maintained their differences in runx-2 expression (Fig. 4n, second to last pair of bars). Therefore, up to passage 6 , the isolated chondrocytes of the TMJ of the DDR-1 KO and WT cells are appropriate for in vitro investigations of TMD.

\section{Differences of protein patterns of KO and WT cells}

To elucidate ECM and cellular protein expression, we performed western blots at passage 2 . In the absence of DDR-1 (Fig. 5a), the expression levels of two key players in OA, DDR-2 (Fig. 5b) and MMP-13 (Fig. 5c), were increased. DDR-1 KO cells produced $1.8 \times$ more DDR-2 and $1.6 \times$ more MMP-13 than WT cells. Notably, the two described isoforms of DDR-2 [33] were both present in WT cells, whereas the KO chondrocytes exhibited just one stronger band with a lower molecular weight. However, DDR-1 KO cells produced $5.1 \times$ more collagen type I than WT cells (Fig. 5d). Sox-9-, runx-2-, and COMP (Fig. 5e-g) were present in nearly equal amounts in DDR-1 KO and WT chondrocytes. The $\beta$-actin staining indicated the equal loading of the gels (Fig. 5h). Coomassie blue staining was performed to evaluate the overall protein bands (Fig. 5i).

The influence of the three-dimensional alginate matrix, bone morphogenetic proteins (BMPs), laminin-1, and nidogen- 2 and the knockdown of runx-2 on TMJ chondrocytes

Under 3D conditions [27], DDR-1 KO cells still exhibited high mRNA levels of runx-2 and collagen type I (Fig. 6a, b), and lower amounts of sox-9 and aggrecan (Fig. 6c, d). This observation underscores their osteoarthritic nature. However, we additionally stimulated the cells with the chondrogenic factor BMP-6 [34] and the basement membrane components laminin- 1 and nidogen- 2 [8], which are present in the normal pericellular chondrocyte matrix [31]. Laminin-1 and nidogen-2 enhanced the chondrogenesis of 

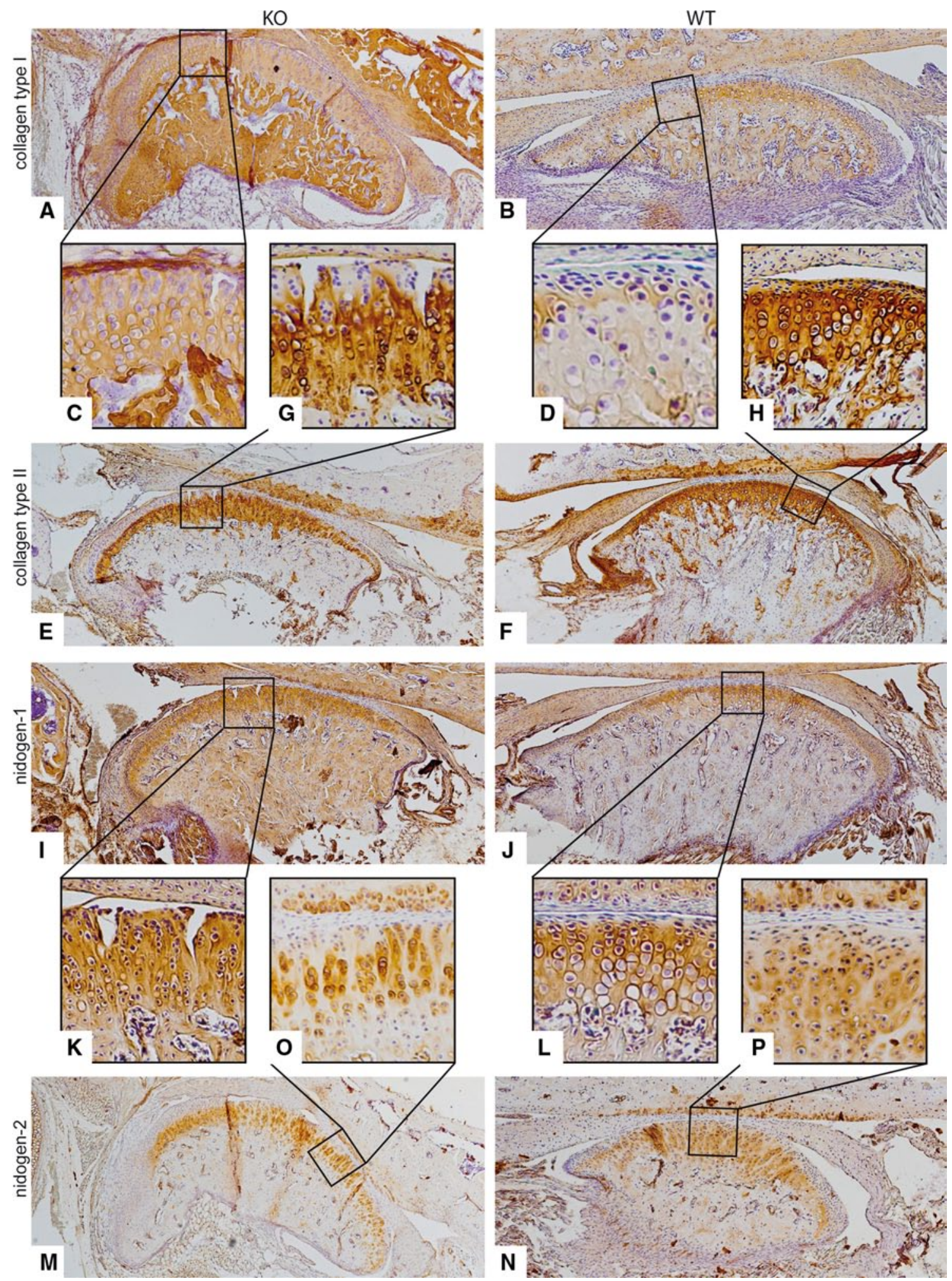

Fig. 2 The molecular changes in the ECM of the TMJ during OA in vivo of 9-week-old mice: Immunohistochemistry was performed for collagen type I $(\mathbf{a}-\mathbf{d})$, collagen type II $(\mathbf{e}-\mathbf{h})$, nidogen-1 $(\mathbf{i}-\mathbf{l})$ and nidogen-2 (m-p). a, c DDR-1 KO TMJ stained for collagen type I is shown. Note the fibrocartilaginous tissue as a sign of tissue regeneration in (c). b, d The WT TMJ exhibited the well-known collagen type I staining. e, $\mathbf{g}$ The KO TMJ showed less collagen type II staining than the WT (f, h). i Nidogen-1 is present in both the KO and the WT mice $(\mathbf{j})$. However, there were no differences between the DDR-1 KO mice (k) and the corresponding WT mice (I). m Nidogen-2 staining was stronger in the TMJs of DDR-1-deficient mice than in the TMJs of WT mice (n). o Note the intense pericellular staining in the deeper zones of the DDR-1 KO TMJ cartilage. Less nidogen-2 was present in the pericellular matrix of the WT cartilage (p). The numbers of the animals evaluated at each time point are shown in Tables 1 and 2 

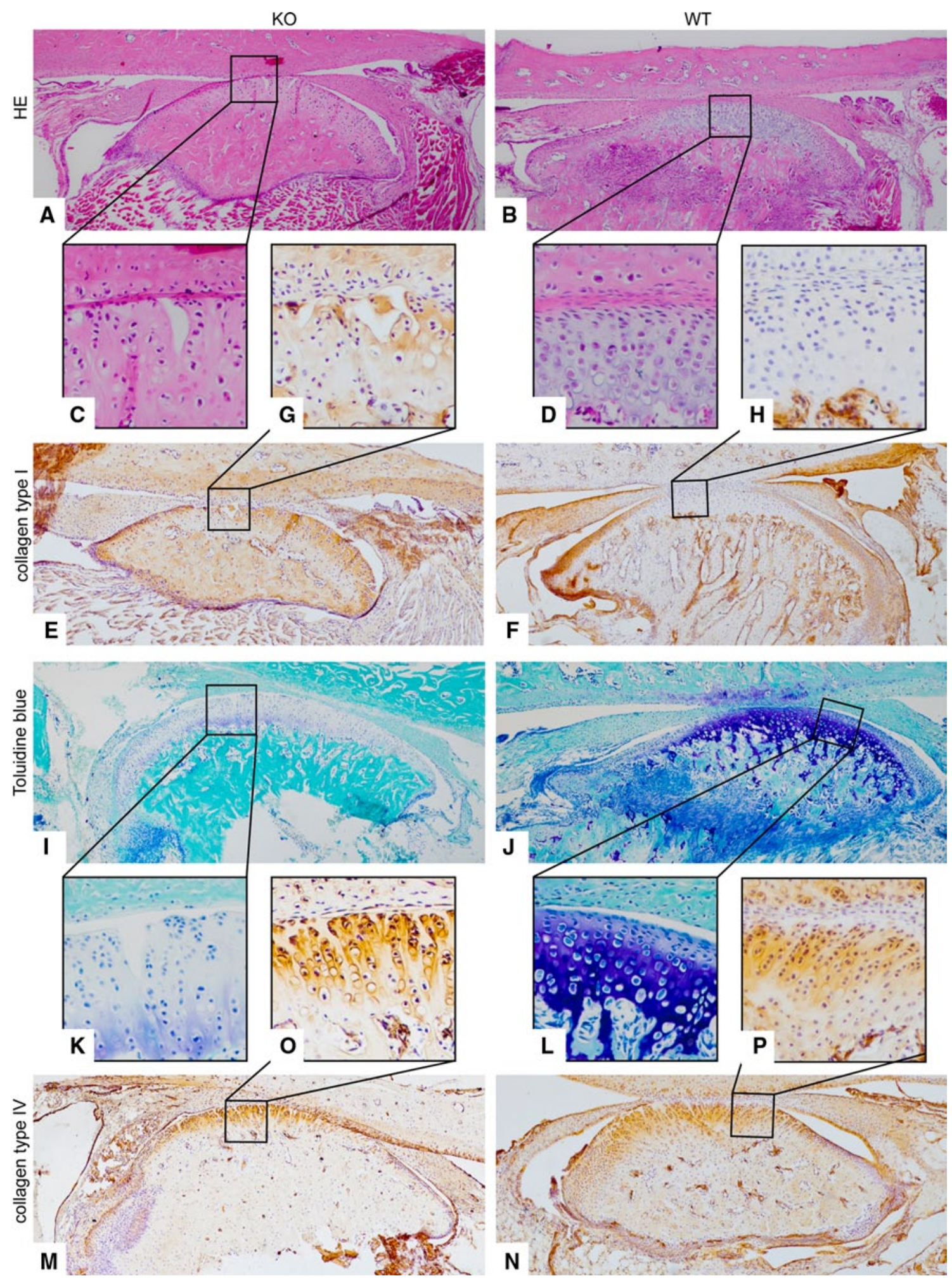

Fig. 3 The histopathology and immunohistology of 24- and 28-weekold mice. a-d HE staining of 24-week-old mice. c Note the surface fissures in the KO compared with the intact joint surface of the WT mice. d Immunohistochemistry results for collagen type I of staining in 28-week-old mice. There was an increase of collagen type I in the KO (e) versus the WT mice (f). The joint surface of the KO is destroyed (g), while the WT joint was smooth and rounded shaped (h). i-l Toluidine blue histology of 24-weeks old mice: the DDR-1
KO condyle exhibited well-known signs of OA, e.g., reduced staining of the superficial zone (i), cluster formation and surface fissure (k); normal WT condyles were observed in $(\mathbf{j}, \mathbf{l})$. m-p Immunohistochemistry results of collagen type IV staining in 28-week-old KO mice: intense staining for collagen type IV was observed at the joint surface $(\mathbf{m})$, especially in the pericellular matrix (o); nNormal WT staining was observed in $(\mathbf{n}, \mathbf{p})$. The numbers of the animals evaluated at each time point are shown in Tables 1 and 2 

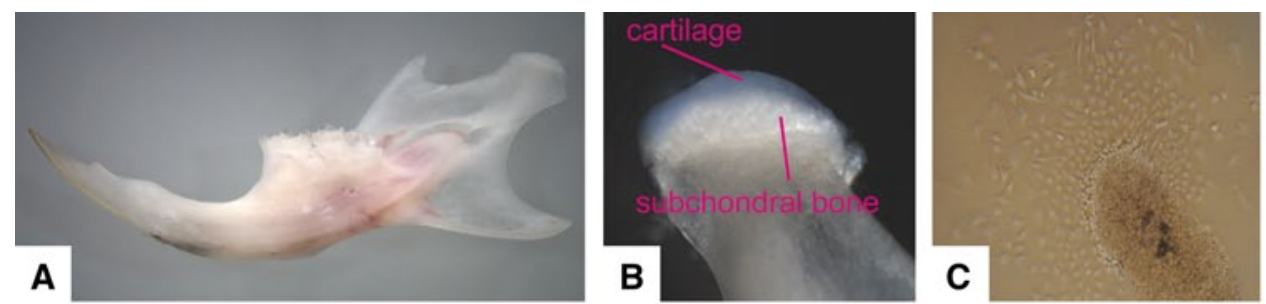

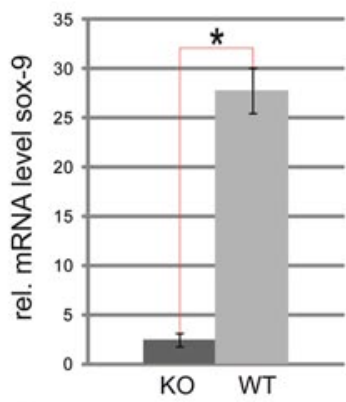

D

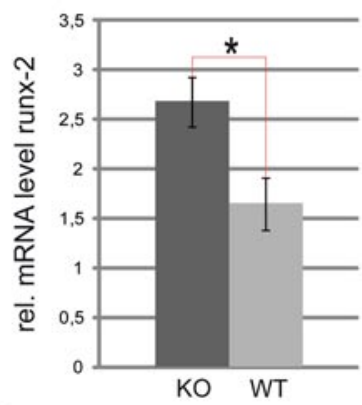

E

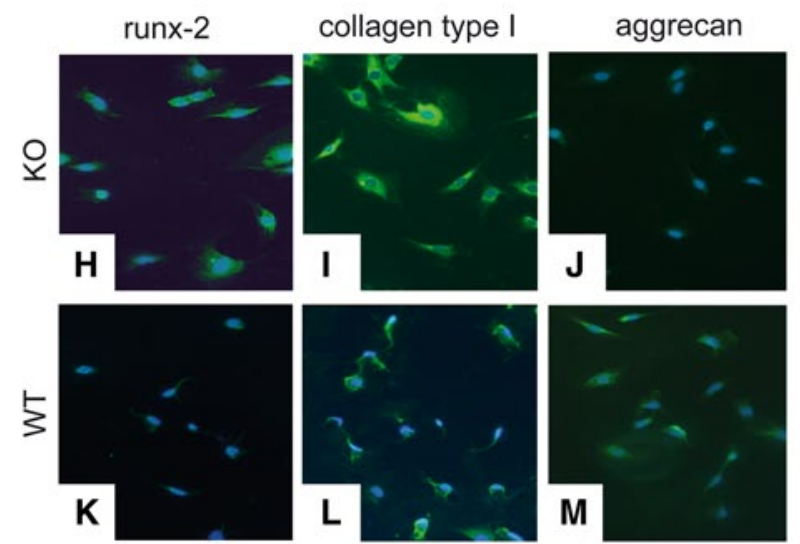

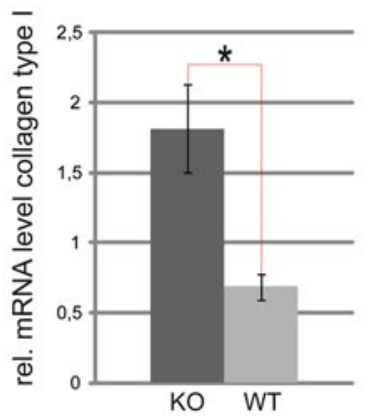

$\mathbf{F}$

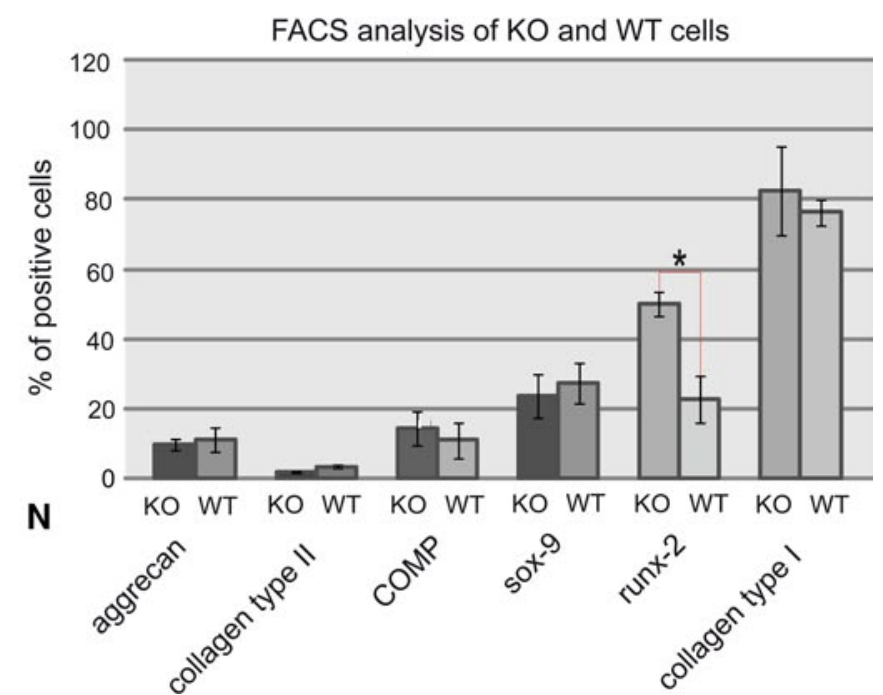

$(\mathbf{i}, \mathbf{l})$, and aggrecan $(\mathbf{j}, \mathbf{m})$ were detected in both KO and WT cells. $\mathbf{n}$ Intracellular FACS analysis of cells in passage 6 identified aggrecan, collagen type II, COMP, and sox-9, as well as the osteoarthritic markers, runx-2 and collagen type I, in both cell types. A higher percentage of DDR-1 KO chondrocytes expressed runx-2 (second-to-last bars). *Significant differences $(p \leq 0.05)$; data are mean values with SD of three individual experiments $(n=10$, including $4 \mathrm{KO}$ mice, $4 \mathrm{WT}$ mice, and 2 controls, for mRNA measurements; $n=6$, including 3 KO mice and 3 WT mice, for immunocytochemistry and FACS-analysis)

[12]. Therefore, we tested whether the knockdown of runx-2 would influence the chondrogenic potential of DDR-1 KO chondrocytes. The transient knockdown of runx-2 mRNA resulted in the complete loss of the runx-2 protein at $24 \mathrm{~h}$ (Fig. 6i). The same expression pattern was observed for collagen type I (Fig. 6j). The chondrogenic potential of DDR1-deficient chondrocytes was improved with the knockdown 

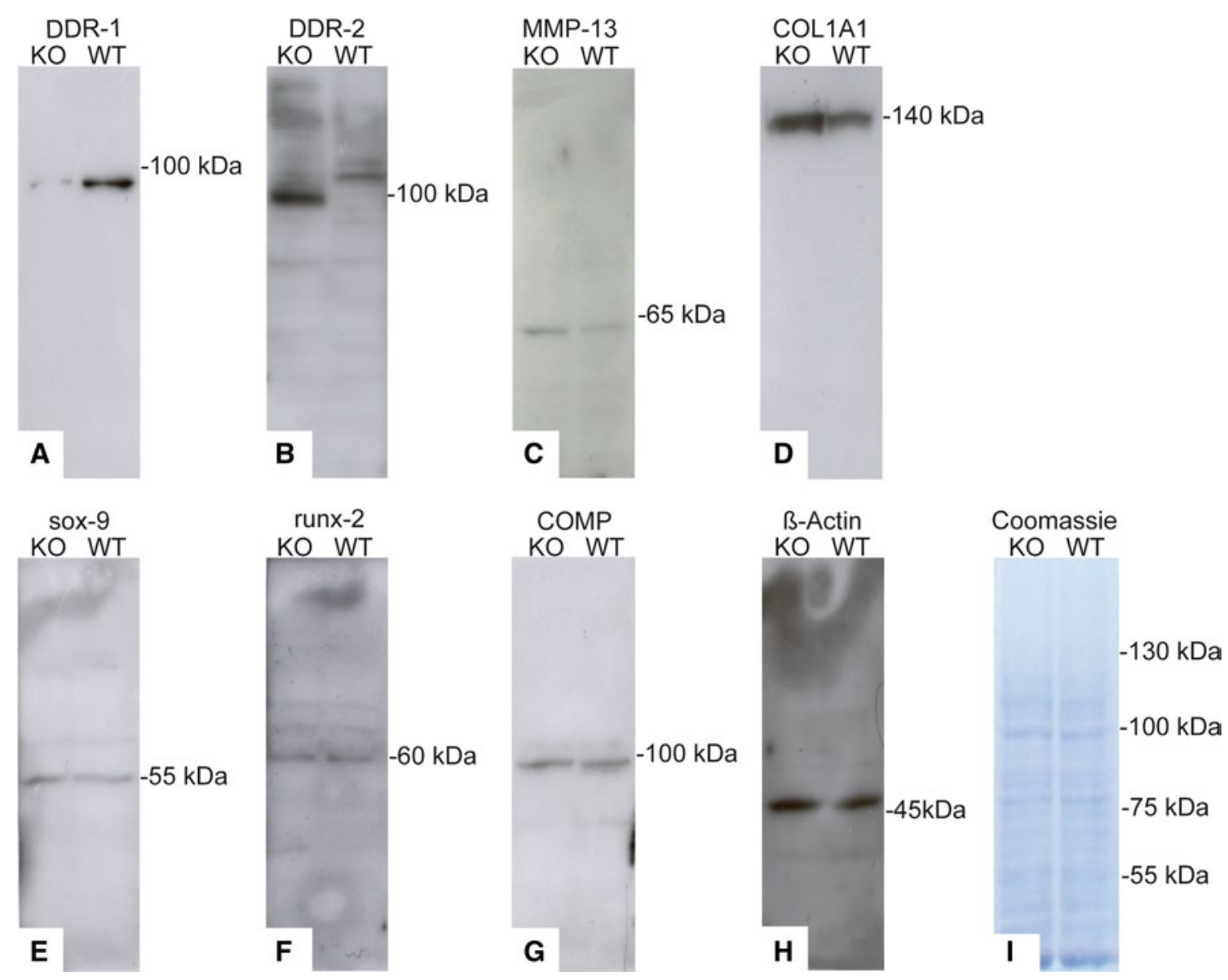

Fig. 5 Differences in protein patterns in KO and WT cells. a The western blot for DDR-1 in the DDR-1 KO confirmed its absence; however, DDR-2 (b), MMP-13 (c) and collagen type I (d) were upregulated in the KO chondrocytes. Sox-9 (e), runx-2 (f) and COMP (g) were present in both the KO and WT cells. $\mathbf{h} \beta$-actin staining confirmed the equal loading of the gels. i Coomassie blue staining was

performed to evaluate the overall protein patterns. Protein isolation was performed using cells at passage 2 of the cells. Data are representatives of three individual experiments, or quantified as stated in "Results" ( $n=6$, including $3 \mathrm{KO}$ mice and $3 \mathrm{WT}$ mice, for western blots)

of runx-2, consistent with the $1.8 \times$ higher amounts of sox-9 (Fig. 6k) and $3.4 \times$ greater collagen type II expression (Fig. 61). Tubulin staining was assessed to confirm the equal loading of the gels (Fig. $6 \mathrm{~m}$ ). The key role of runx-2 as a transcription factor of the osteoblastic lineage, was demonstrated by its localization in the nucleus (Supplemental Fig. 1a-c) and the overexpression of runx-2 in the cells (Supplemental Fig. 1d, e). The overexpression resulted in the enhanced gene expression of the downstream mediators Col1A1, SPP1 and IBSP (Supplemental Fig. 1f-i).

Pathways involved in OA of the TMJ and the downstream signaling of DDRs

We detected an upregulation of hedgehog interacting protein (HHIP), which is known to be involved in OA pathogenesis [35]. We also found that vascular endothelial growth factor A (VEGFA), which is associated with the
Wnt pathway in OA [36], was increased in DDR-1 KO chondrocytes (Fig. 7a). The loss of DDR-1, with its concomitant increase in DDR-2, initiates an upregulation of MMP-13 (Fig. 4c), presumably resulting in the degradation of collagens, mainly type II [17], in TMJ OA. Pit is possible that downstream signaling involves players of the $\mathrm{IHH}$ and Wnt pathways (Fig. 7a, b).

\section{Investigation of the primary cilia in TMJ chondrocytes}

It has been established that ECM proteins, mainly collagens, are responsible for the transduction of forces within the cartilage tissue; this is essential for skeletal growth [37]. The main cellular mediator of mechanosensing in chondrocytes is the primary cilium [38]. The microarray data showed that many of the regulated genes with altered expression in the DDR-1 KO were associated with the primary cilia (Supplemental Fig. 2a). Surprisingly, we observed fewer cells with 

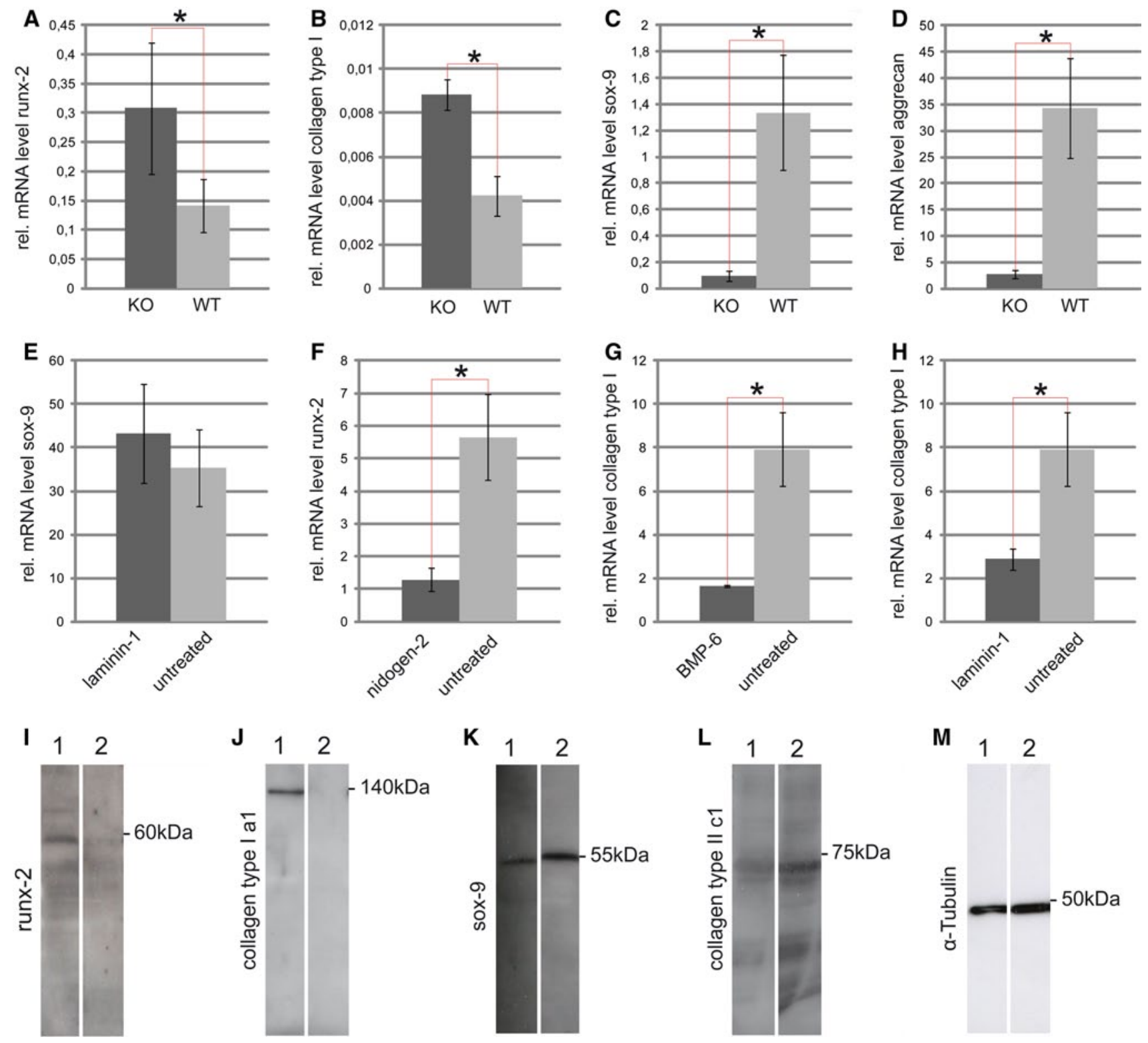

Fig. 6 The influence of three-dimensional alginate matrix, BMPs, laminin-1, nidogen-2 and the knockdown of runx-2 on TMJ chondrocytes: The expression patterns of runx-2 (a), collagen type I (b), sox-9 (c) and aggrecan (d) were similar to the results obtained in 2D-cultured cells (Fig. 3d-g). e Laminin-1 stimulation resulted in an upregulation of sox-9 in DDR-1 KO chondrocytes, while nidogen-2 down-regulates runx-2 (f). Therefore, the two basement membrane components promote the chondrogenesis of these cells. g BMP-6 reduced the relative mRNA levels of collagen type I in DDR-1 KO chondrocytes, as did laminin-1 (h), which is also a marker of chondrogenic differentiation. i Runx-2 protein expression was not detect-

primary cilia among the osteoarthritic DDR-1 KO chondrocytes (Supplemental Fig. 2b, upper panel) than among the WT chondrocytes (Supplemental Fig. 2b, lower panel); however, the differences in numbers did not reach statistical significance (Supplemental Fig. 2c). able $24 \mathrm{~h}$ (lane 2) after the knockdown performed by the transient transfection with the siRNA vector. Lane 1 always represents the control. j Collagen type I was not detectable 24 h (lane 2) after runx-2 knockdown, as expected; $\mathbf{k}$ the amount of sox-9 was elevated compared with the control cells (lane 1). I Collagen type II was detectable (lane 2), after runx-2 knockdown, but not in the control cells, in which collagen type II was not detectable (lane 1). m Tubulin staining confirmed the equal loading of the gels. $\mathbf{k}-\mathbf{m}$ are composite figures. *Significant differences $(p \leq 0.05)$; data are mean values with SD from three individual experiments. $(n=10$, including $4 \mathrm{KO}$ mice, 4 WT mice and 2 controls, for mRNA measurements)

\section{Discussion}

The present study introduces the DDR-1 null mouse as a new model for OA of the TMJ. These mice develop OA more frequently and at a younger age than other mouse models 
Fig. 7 Possible players downstream of the DDRs in OA of the TMJ. a A PCR array identified the regulated signaling pathway molecules, with the IHH pathway prominently involved in SSH and BMP-2 interactions. VEGFA upregulation was observed. b Surface fissures $(a)$ and proteoglycan degradation $(b)$ of the TMJ of the DDR-1 KO mice revealed typical OA characteristics. The pathomechanism of OA in the TMJ of DDR-1 KO mice results in an upregulation of runx-2, collagen type I and DDR-2 (1). This leads to an increased activation of MMP-13 (2) to enhance matrix degradation, especially of collagens (3). *Also downregulated: Naip1, Brca1, Ccl2, Ccl20, Cd5, Cdh1, Csf2, Cxc11, Cxc19, Cyp19a1, En1, Fasl, Fgf4, Greb1, Hoxa1, Icam1, Il1a, Il2, Il2ra, Lef1, Lep, Lta, Mmp10, Mmp7, Nos2, Pparg, Rbp1, Tnf, Wnt1, Wnt2, MGDC, and Selp. Data are representative of three individual experiments. $(n=6$, including $3 \mathrm{KO}$ mice and $3 \mathrm{WT}$ mice, for PCR array)

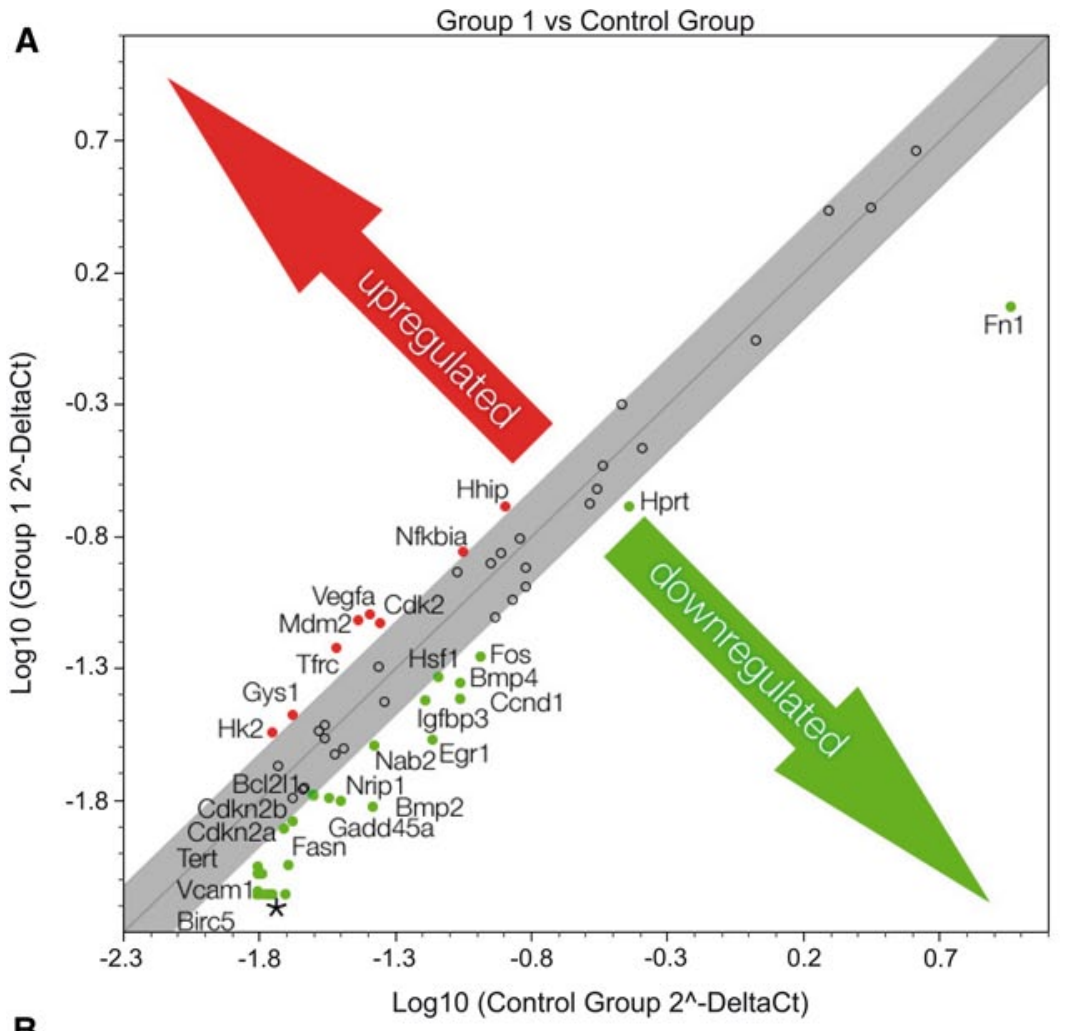

B

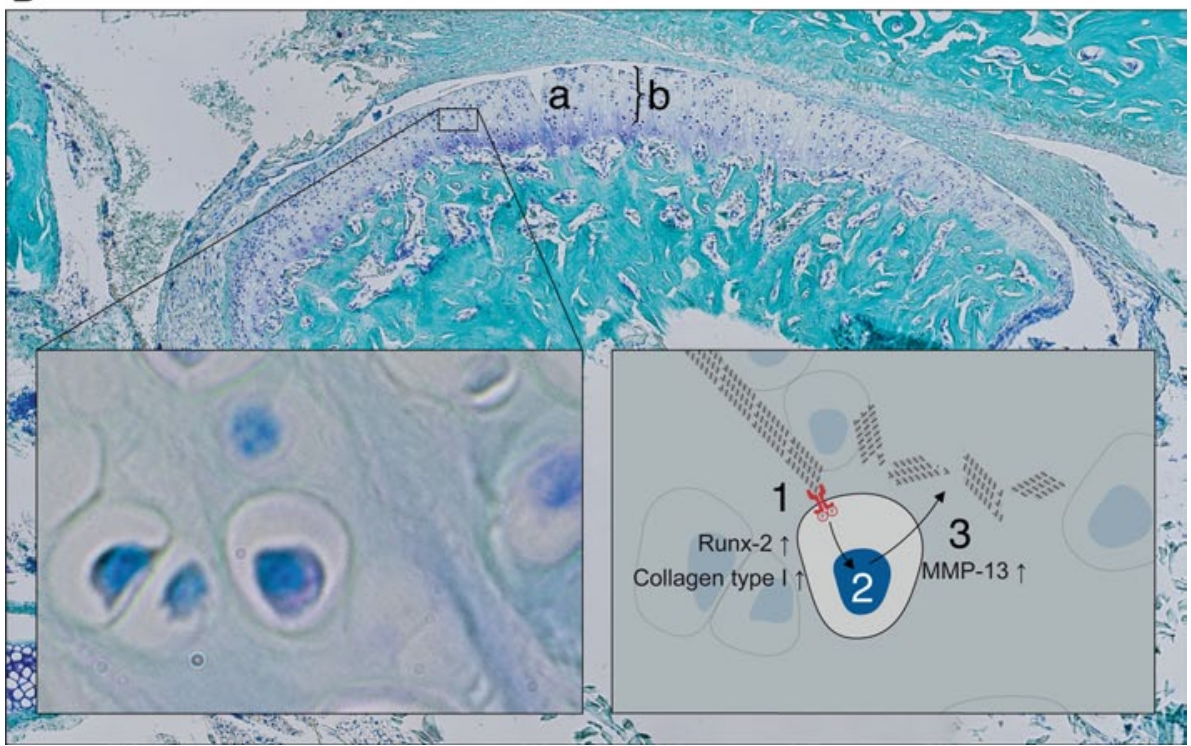

currently used to study TMD, including the ICR mouse [14], the Del 1 mouse [15], and the Cho mouse [16]. The loss of DDR-1 expression has several significant consequences that influence OA pathogenesis, including increased DDR-2, MMP-13, collagen type I, and runx-2 expression.

The pathomechanism of the DDR-1 KO mice

We applied several approaches to characterize the articular chondrocytes from DDR-1 KO TMJs. Using a combination of real-time RT-PCR, immunocytochemistry, and FACS analysis, we found that DDR1-deficient chondrocytes exhibited characteristics of osteoarthritis. The observed differences between the mRNA and protein levels of certain factors (Fig. 4) are not completely understood. There are a wide variety of post-transcriptional regulatory processes; for example, it is possible that micro RNAs [39] or catabolic enzymes such as RNase [40] play an important role in the cartilage biology of the TMJ cartilage. The KO chondrocytes produced high amounts of collagen type I and 
runx-2 as well as low levels of collagen type II and sox9. These changes in gene expression promoted the development of an osteoarthritic phenotype [41]. Most significantly, DDR-1 KO chondrocytes displayed a compensatory increase in the expression of a DDR-2 isoform, and this receptor is linked to elevated MMP-13 (Fig. 5c) expression [42, 43] and articular cartilage degeneration [44]. Using signaling arrays, we determined that DDR-1-null chondrocytes exhibited increased expression of IHH signaling pathway components, as measured by increases in HHIP [35], a protein reported to be overexpressed in human OA and in cartilage from other OA mouse models. Hydrostatic compression of the chondrocytic primary cilia, which is essential for cartilage mechanotransduction [45], upregulates IHH gene expression [46]. It is also known that mechanical stimulation upregulates IHH expression in chondrocytes and is associated with OA [47]. We found a slight tendency towards a reduction of the primary cilia in the osteoarthritic TMJ chondrocytes. We, therefore, only speculate that primary cilia in the DDR-1-deficient chondrocytes might be involved in the pathogenesis.

\section{Why does the lack of DDR-1 result in OA of the TMJ?}

Although DDR-1-deficient mice develop OA of the TMJ by 9 weeks of age, we did not detect any signs of OA in the knee joints of mice at this age. In fact, few DDR-1 KO mice developed OA of the large joints. One possible explanation for this finding is the structural difference between the two joints. Unlike the articular cartilage of the knee, the cartilage of the mandibular condyle is considered a secondary cartilage $[48,49]$ and has a different embryonic origin. In addition, the molecular composition of the TMJ differs from that of the larger synovial joints in that it contains large amounts of collagen type I [50], especially in the marginal areas of the joint where the joint capsule and associated ligaments are found. Furthermore, in contrast to the articular cartilage of other joints, the superficial layer of the mandibular condylar cartilage does not normally express collagen type II, although the functional significance of this difference is unknown [1]. Despite these structural differences, the absence of DDR-1 further reduced the amount of collagen type II and increased the amount of MMP-13 within the TMJ, thereby promoting the early development of OA (Fig. 7b).

\section{DDR-1-deficient chondrocytes and cartilage regeneration}

Interestingly, we observed areas of regeneration on the articular surface of the degenerating jaws of DDR-1 KO mice. These areas displayed intense staining for collagen type I, similar to that observed in OA in the human knee joint [51]. In the pericellular space, especially that of cell clusters, an upregulation of nidogen-2 and collagen type IV was observed. This is also an indication of regenerative efforts within the diseased cartilage tissue. TMJ chondrocytes, similar to their counterparts in the knee [12], are regulated by runx-2 and sox-9; here, we were able to enhance the chondrogenic potential of the DDR-1-deficient chondrocytes via runx-2 knockdown. The runx-2 knockdown resulted in an increased expression of sox-9, which then stimulates the enhanced expression of collagen type II and reduced levels of collagen type I. Similar effects were also shown for aggrecan [52] and COMP [53]. Therefore, the chondrogenic potential is enhanced as DDR-1-deficient chondrocytes lose their osteoarthritic character via the runx-2 knockdown. Another target for the regeneration of TMJ cartilage is the DDR-2 receptor. It is well known that DDR-2 is activated via a direct interaction with collagen type II, which does not appear in the pericellular matrix of the chondrocyte in healthy cartilage, but it does in OA. Therefore, DDR-2 is activated during OA. For example, a mutation in DDR2 , that disrupts its binding to collagen type II, reduces the collagen-induced expression of MMP-13 [54].

Taken together, our findings indicate that the DDR-1-deficient mouse is a novel animal model for the in vivo study of human TMD. Furthermore, the isolated TMJ chondrocytes can be used for the in vitro analysis of pathomechanisms of the TMJ. We show that TMJ cartilage regeneration is controlled by the transcription factors sox- 9 and runx- 2 and is influenced by signals from the pericellular matrix, including BMPs that enhance chondrogenesis. These measures result in a gene expression signature similar to that of normal articular cartilage, suggesting that the DDR-1 KO mouse can serve as a novel model for TMD, such as OA of the TMJ. This model will help to develop and test new treatment options, particularly those involving tissue regeneration.

Acknowledgments The authors would like to thank the staff of the animal facilities at the MPI Experimental Medicine, Goettingen and the Medical Faculty of the University of Goettingen for animal care, Dr. Bunt, Molecular \& Live Cell Imaging (MOLCI), central imaging facility of the UMG, for the confocal microscopy work, Dr. SalinasRiester for performing the microarray and Mr. Opitz for statistical evaluation, and Dr. Dullin for help with the micro-CT. We would also like to thank Mr. Hoehne, as parts of the results were taken from his doctoral thesis. We also wish to thank Mr. Menrath for professional assistance with the figure layout.

\section{References}

1. Wadhwa S, Embree M, Ameye L, Young MF (2005) Mice deficient in biglycan and fibromodulin as a model for temporomandibular joint osteoarthritis. Cells Tissues Organs 181(3-4):136143. doi: $10.1159 / 000091375$

2. Carlson GE, Magnusson T (1999) Management of temporomandibular disorders in the general dental practice, vol 1. Quintessenz, Berlin 
3. Lohmander LS, Roos EM (2007) Clinical update: treating osteoarthritis. Lancet 370(9605):2082-2084. doi:10.1016/ s0140-6736(07)61879-0

4. Buckwalter JA, Mankin HJ (1998) Articular cartilage: degeneration and osteoarthritis, repair, regeneration, and transplantation. Instr Course Lect 47:487-504

5. Horton WE Jr, Bennion P, Yang L (2006) Cellular, molecular, and matrix changes in cartilage during aging and osteoarthritis. J Musculoskel Neuronal Interact 6(4):379-381

6. Poole AR (1999) An introduction to the pathophysiology of osteoarthritis. Front Biosci 4:D662-D670

7. Sandell LJ (2007) Modern molecular analysis of a traditional disease: progression in osteoarthritis. Arthr Rheum 56(8):24742477. doi:10.1002/art.22760

8. Kruegel J, Sadowski B, Miosge N (2008) Nidogen-1 and nidogen-2 in healthy human cartilage and in late-stage osteoarthritis cartilage. Arthr Rheum 58(5):1422-1432. doi:10.1002/art.23480

9. Kuettner KE (1992) Biochemistry of articular cartilage in health and disease. Clin Biochem 25(3):155-163

10. Loeser RF (2000) Chondrocyte integrin expression and function. Biorheology 37(1-2):109-116

11. Goldring MB, Otero M (2011) Inflammation in osteoarthritis. Curr Opin Rheumatol 23(5):471-478. doi:10.1097/BOR.0b013e $328349 \mathrm{c} 2 \mathrm{~b} 1$

12. Koelling S, Kruegel J, Irmer M, Path JR, Sadowski B, Miro X, Miosge N (2009) Migratory chondrogenic progenitor cells from repair tissue during the later stages of human osteoarthritis. Cell Stem Cell 4(4):324-335. doi:10.1016/j.stem.2009.01.015

13. Seol D, McCabe DJ, Choe H, Zheng H, Yu Y, Jang K, Walter MW, Lehman AD, Ding L, Buckwalter JA, Martin JA (2012) Chondrogenic progenitor cells respond to cartilage injury. Arthr Rheum 64(11):3626-3637. doi:10.1002/art.34613

14. Silbermann M, Livne E (1979) Age-related degenerative changes in the mouse mandibular joint. J Anat 129(Pt 3):507-520

15. Rintala M, Metsaranta M, Saamanen AM, Vuorio E, Ronning O (1997) Abnormal craniofacial growth and early mandibular osteoarthritis in mice harbouring a mutant type II collagen transgene. J Anat 190(Pt 2):201-208

16. Xu L, Flahiff CM, Waldman BA, Wu D, Olsen BR, Setton LA, Li Y (2003) Osteoarthritis-like changes and decreased mechanical function of articular cartilage in the joints of mice with the chondrodysplasia gene (cho). Arthr Rheum 48(9):2509-2518. doi:10.1002/art.11233

17. Xu L, Polur I, Servais JM, Hsieh S, Lee PL, Goldring MB, $\mathrm{Li} Y$ (2011) Intact pericellular matrix of articular cartilage is required for unactivated discoidin domain receptor 2 in the mouse model. Am J Pathol 179(3):1338-1346. doi:10.1016/j.ajpath.2011.05.023

18. Lam NP, Li Y, Waldman AB, Brussiau J, Lee PL, Olsen BR, Xu L (2007) Age-dependent increase of discoidin domain receptor 2 and matrix metalloproteinase 13 expression in temporomandibular joint cartilage of type IX and type XI collagen-deficient mice. Arch Oral Biol 52(6):579-584. doi:10.1016/j.archoral bio.2006.10.014

19. Vogel W (1999) Discoidin domain receptors: structural relations and functional implications. FASEB J 13(Suppl):S77-S82

20. Vogel W, Gish GD, Alves F, Pawson T (1997) The discoidin domain receptor tyrosine kinases are activated by collagen. Mol Cell 1(1):13-23

21. Foehr ED, Tatavos A, Tanabe E, Raffioni S, Goetz S, Dimarco E, De Luca M, Bradshaw RA (2000) Discoidin domain receptor 1 (DDR1) signaling in PC12 cells: activation of juxtamembrane domains in PDGFR/DDR/TrkA chimeric receptors. FASEB J 14(7):973-981

22. Vogel WF, Aszodi A, Alves F, Pawson T (2001) Discoidin domain receptor 1 tyrosine kinase has an essential role in mammary gland development. Mol Cell Biol 21(8):2906-2917. doi:10.112 8/MCB.21.8.2906-2917.2001

23. Little C, Smith S, Ghosh P, Bellenger C (1997) Histomorphological and immunohistochemical evaluation of joint changes in a model of osteoarthritis induced by lateral meniscectomy in sheep. J Rheumatol 24(11):2199-2209

24. Hedbom E, Antonsson P, Hjerpe A, Aeschlimann D, Paulsson M, Rosa-Pimentel E, Sommarin Y, Wendel M, Oldberg A, Heinegard D (1992) Cartilage matrix proteins. An acidic oligomeric protein (COMP) detected only in cartilage. J Biol Chem 267(9):6132-6136

25. Koelling S, Clauditz TS, Kaste M, Miosge N (2006) Cartilage oligomeric matrix protein is involved in human limb development and in the pathogenesis of osteoarthritis. Arthr Res Ther 8(3):R56. doi:10.1186/ar1922

26. Gersdorff N, Kohfeldt E, Sasaki T, Timpl R, Miosge N (2005) Laminin gamma 3 chain binds to nidogen and is located in murine basement membranes. J Biol Chem 280(23):2214622153. doi:10.1074/jbc.M501875200

27. Hauselmann HJ, Fernandes RJ, Mok SS, Schmid TM, Block JA, Aydelotte MB, Kuettner KE, Thonar EJ (1994) Phenotypic stability of bovine articular chondrocytes after long-term culture in alginate beads. J Cell Sci 107(Pt 1):17-27

28. Diaz-Romero J, Gaillard JP, Grogan SP, Nesic D, Trub T, MainilVarlet P (2005) Immunophenotypic analysis of human articular chondrocytes: changes in surface markers associated with cell expansion in monolayer culture. J Cell Physiol 202(3):731-742. doi:10.1002/jcp. 20164

29. Pfaffl MW (2001) A new mathematical model for relative quantification in real-time RT-PCR. Nucleic Acids Res 29(9):e45

30. Goldring MB, Goldring SR (2010) Articular cartilage and subchondral bone in the pathogenesis of osteoarthritis. Ann NY Acad Sci 1192:230-237. doi:10.1111/j.1749-6632.2009.05240.x

31. Kvist AJ, Nystrom A, Hultenby K, Sasaki T, Talts JF, Aspberg A (2008) The major basement membrane components localize to the chondrocyte pericellular matrix - a cartilage basement membrane equivalent? Matrix Biol 27(1):22-33. doi:10.1016/j.matbio.2007.07.007

32. Holtzer H, Abbott J, Lash J, Holtzer S (1960) The loss of phenotypic traits by differentiated cells in vitro, I. Dedifferentiation of cartilage cells. Proc Natl Acad Sci USA 46(12):1533-1542

33. Ruiz PA, Jarai G (2011) Collagen I induces discoidin domain receptor (DDR) 1 expression through DDR2 and a JAK2-ERK1/2mediated mechanism in primary human lung fibroblasts. J Biol Chem 286(15):12912-12923. doi:10.1074/jbc.M110.143693

34. Bandyopadhyay A, Tsuji K, Cox K, Harfe BD, Rosen V, Tabin CJ (2006) Genetic analysis of the roles of BMP2, BMP4, and BMP7 in limb patterning and skeletogenesis. PLoS Genet 2(12):e216. doi:10.1371/journal.pgen.0020216

35. Lin AC, Seeto BL, Bartoszko JM, Khoury MA, Whetstone H, Ho L, Hsu C, Ali SA, Alman BA (2009) Modulating hedgehog signaling can attenuate the severity of osteoarthritis. Nat Med 15(12):1421-1425. doi:10.1038/nm.2055

36. Enomoto H, Inoki I, Komiya K, Shiomi T, Ikeda E, Obata K, Matsumoto H, Toyama Y, Okada Y (2003) Vascular endothelial growth factor isoforms and their receptors are expressed in human osteoarthritic cartilage. Am J Pathol 162(1):171-181

37. Reich A, Maziel SS, Ashkenazi Z, Ornan EM (2010) Involvement of matrix metalloproteinases in the growth plate response to physiological mechanical load. J Appl Physiol 108(1):172-180. doi:10.1152/japplphysiol.00821.2009

38. Whitfield JF (2008) The solitary (primary) cilium-a mechanosensory toggle switch in bone and cartilage cells. Cell Signal 20(6):1019-1024. doi:10.1016/j.cellsig.2007.12.001

39. Martinez-Sanchez A, Dudek KA, Murphy CL (2012) Regulation of human chondrocyte function through direct inhibition of cartilage 
master regulator SOX9 by microRNA-145 (miRNA-145). J Biol Chem 287(2):916-924. doi:10.1074/jbc.M111.302430

40. Mattijssen S, Welting TJ, Pruijn GJ (2010) RNase MRP and disease. Wiley Interdiscip Rev RNA 1(1):102-116. doi:10.1002/wrna.9

41. Kamekura S, Kawasaki Y, Hoshi K, Shimoaka T, Chikuda H, Maruyama Z, Komori T, Sato S, Takeda S, Karsenty G, Nakamura K, Chung UI, Kawaguchi H (2006) Contribution of runtrelated transcription factor 2 to the pathogenesis of osteoarthritis in mice after induction of knee joint instability. Arthr Rheum 54(8):2462-2470. doi:10.1002/art.22041

42. Xu L, Peng H, Wu D, Hu K, Goldring MB, Olsen BR, Li Y (2005) Activation of the discoidin domain receptor 2 induces expression of matrix metalloproteinase 13 associated with osteoarthritis in mice. J Biol Chem 280(1):548-555. doi:10.1074/jbc. M411036200

43. Otero M, Plumb DA, Tsuchimochi K, Dragomir CL, Hashimoto K, Peng H, Olivotto E, Bevilacqua M, Tan L, Yang Z, Zhan Y, Oettgen P, Li Y, Marcu KB, Goldring MB (2012) E74-like factor 3 (ELF3) impacts on matrix metalloproteinase 13 (MMP13) transcriptional control in articular chondrocytes under proinflammatory stress. J Biol Chem 287(5):3559-3572. doi:10.1074/jbc. M111.265744

44. Sunk IG, Bobacz K, Hofstaetter JG, Amoyo L, Soleiman A, Smolen J, Xu L, Li Y (2007) Increased expression of discoidin domain receptor 2 is linked to the degree of cartilage damage in human knee joints: a potential role in osteoarthritis pathogenesis. Arthr Rheum 56(11):3685-3692. doi:10.1002/art.22970

45. Wann AK, Zuo N, Haycraft CJ, Jensen CG, Poole CA, McGlashan SR, Knight MM (2012) Primary cilia mediate mechanotransduction through control of ATP-induced $\mathrm{Ca}^{2+}$ signaling in compressed chondrocytes. FASEB J 26(4):1663-1671. doi:10.1096/fj.11-193649
46. Shao YY, Wang L, Welter JF, Ballock RT (2012) Primary cilia modulate Ihh signal transduction in response to hydrostatic loading of growth plate chondrocytes. Bone 50(1):79-84. doi:10.1016/j.bone.2011.08.033

47. Chang CF, Ramaswamy G, Serra R (2012) Depletion of primary cilia in articular chondrocytes results in reduced Gli3 repressor to activator ratio, increased Hedgehog signaling, and symptoms of early osteoarthritis. Osteoarthr Cartil 20(2):152-161. doi:10.1016/j.joca.2011.11.009

48. Symons NB (1965) A histochemical study of the secondary cartilage of the mandibular condyle in the rat. Arch Oral Biol 10(4):579-584

49. Shen G, Darendeliler MA (2005) The adaptive remodeling of condylar cartilage - a transition from chondrogenesis to osteogenesis. J Dent Res 84(8):691-699

50. Benjamin M, Ralphs JR (2004) Biology of fibrocartilage cells. Int Rev Cytol 233:1-45. doi:10.1016/s0074-7696(04)33001-9

51. Koelling S, Miosge N (2010) Sex differences of chondrogenic progenitor cells in late stages of osteoarthritis. Arthr Rheum 62(4):1077-1087. doi:10.1002/art.27311

52. Bi W, Deng JM, Zhang Z, Behringer RR, de Crombrugghe B (1999) Sox9 is required for cartilage formation. Nat Genet 22(1):85-89. doi:10.1038/8792

53. Tallheden T, Karlsson C, Brunner A, Van Der Lee J, Hagg R, Tommasini R, Lindahl A (2004) Gene expression during redifferentiation of human articular chondrocytes. Osteoarthr Cartil 12(7):525-535. doi:10.1016/j.joca.2004.03.004

54. Xu L, Peng H, Glasson S, Lee PL, Hu K, Ijiri K, Olsen BR, Goldring MB, Li Y (2007) Increased expression of the collagen receptor discoidin domain receptor 2 in articular cartilage as a key event in the pathogenesis of osteoarthritis. Arthr Rheum 56(8):2663-2673. doi:10.1002/art.22761 


\section{Chapter 3}

A discoidin domain receptor 1 knockout mouse as a novel model for osteoarthritis of the temporomandibular joint

Supplemental data 

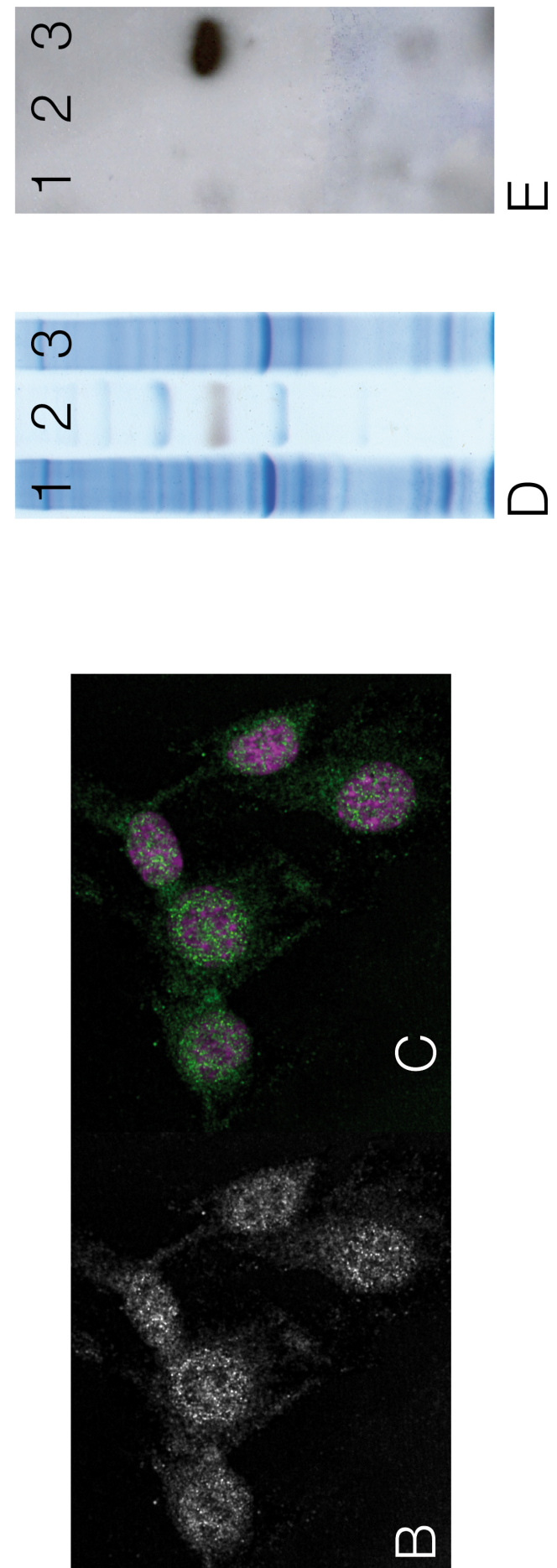

$\varangle$
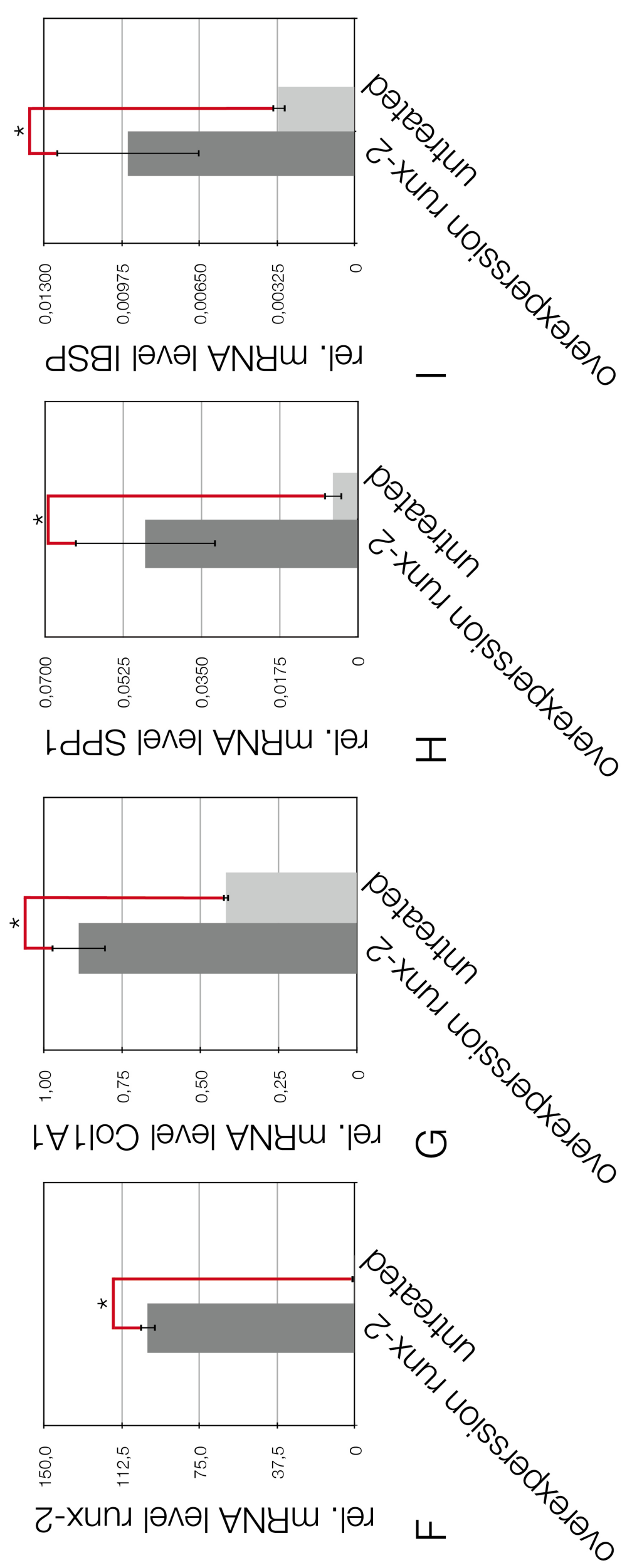

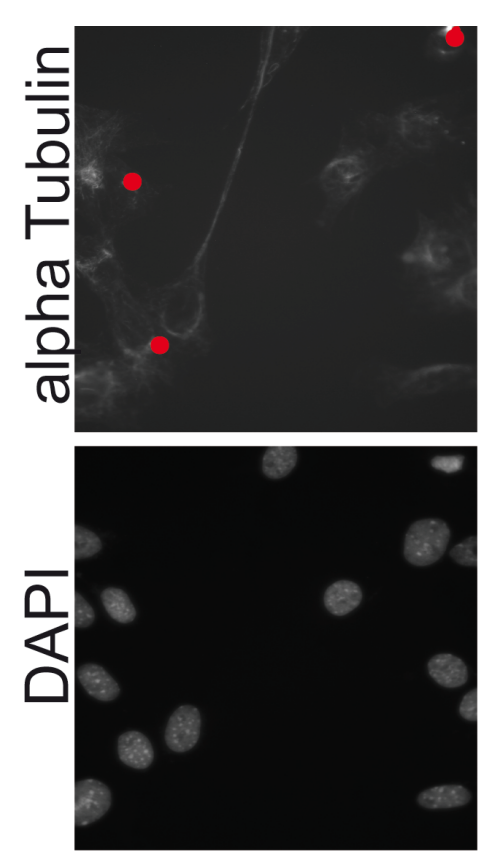

OX
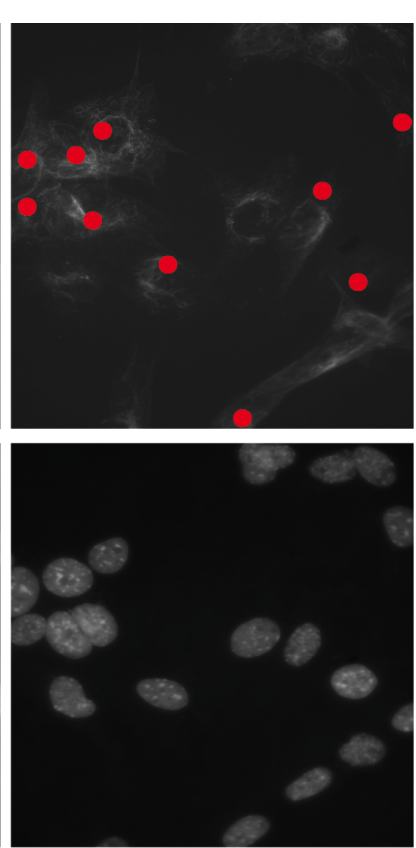

$\perp M$

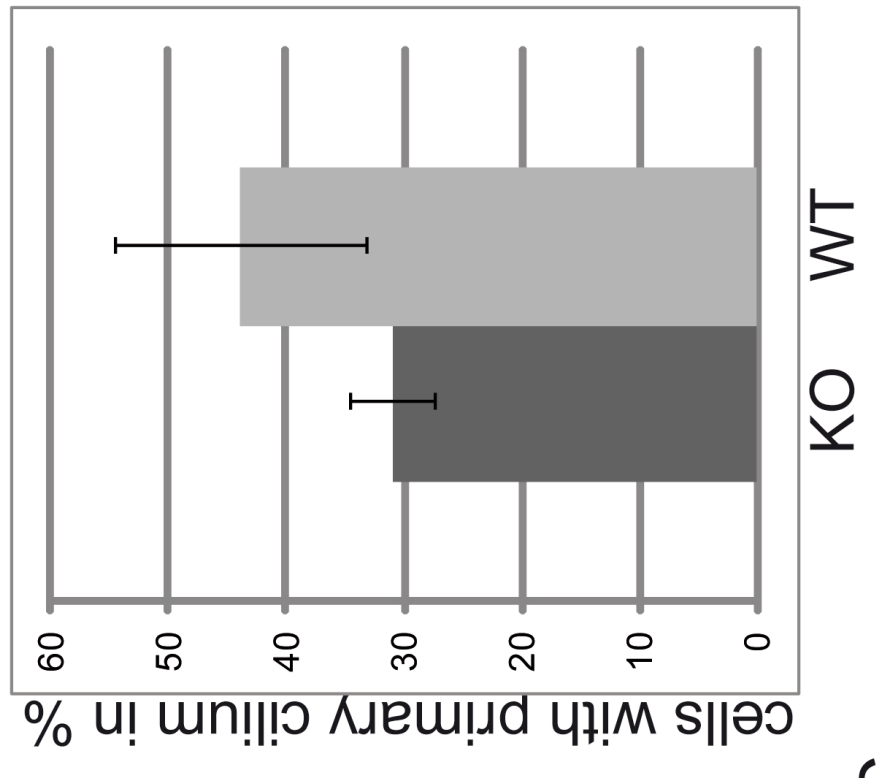

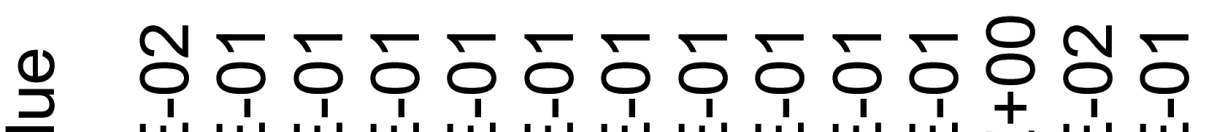
ய山 山ய

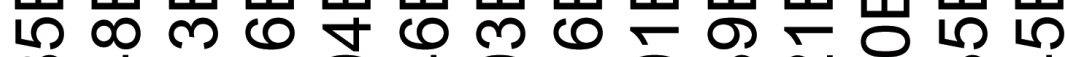

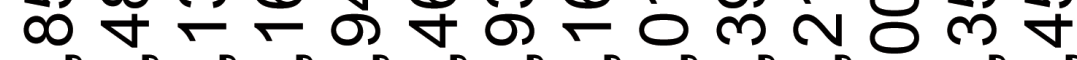

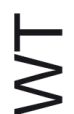
G F

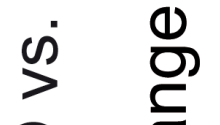

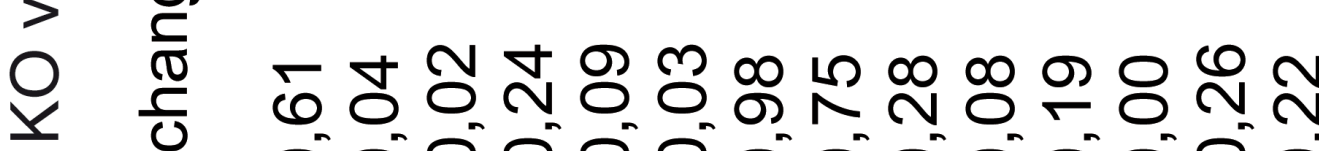
$\therefore \quad$ 응

(1)

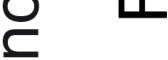

(1)

选 巳 응 凹

यु

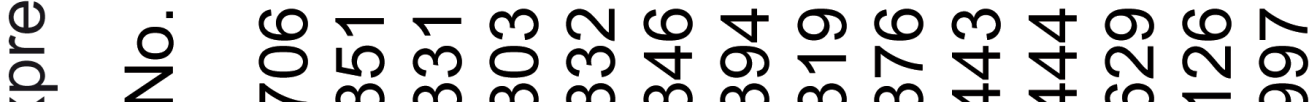

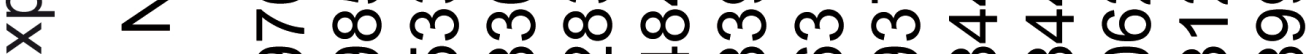
대 은 $8008 \%$

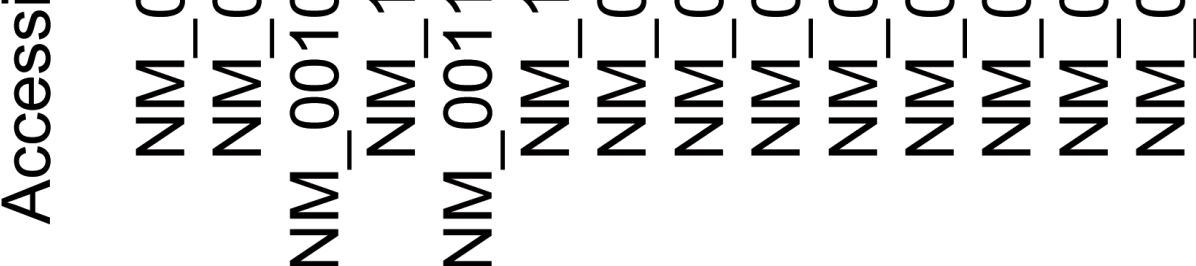




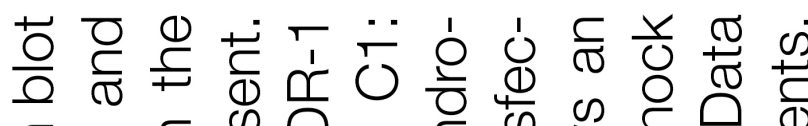

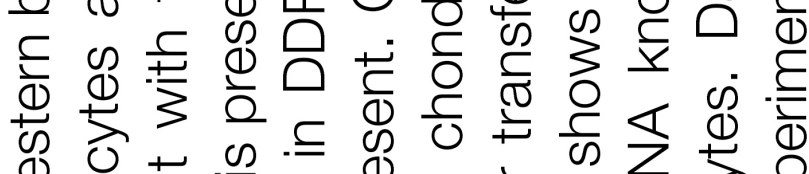

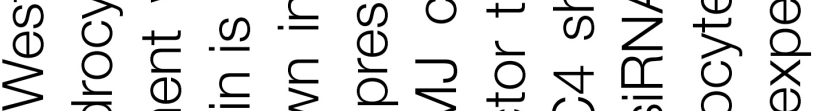

这.

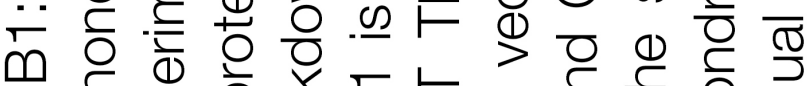

心

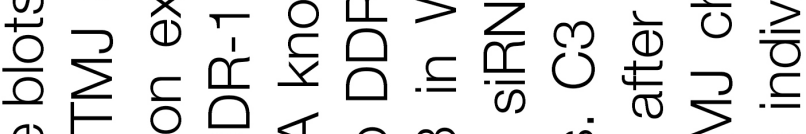

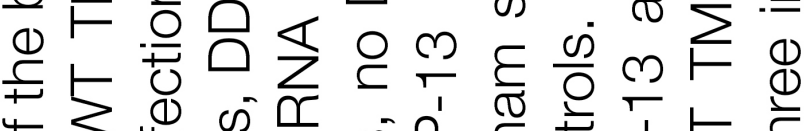

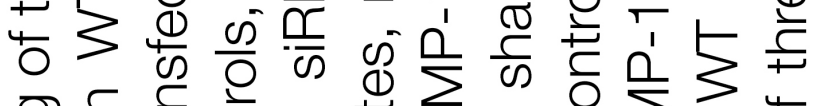

马. .

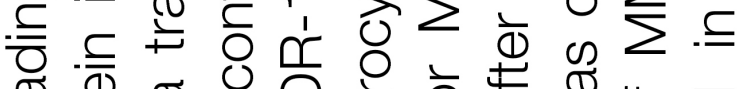

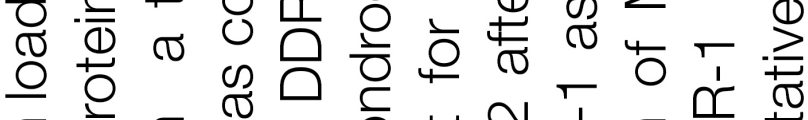

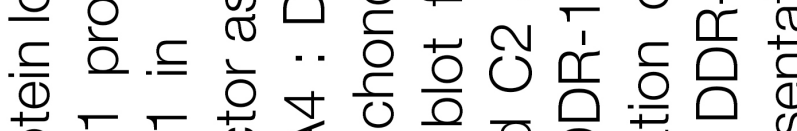

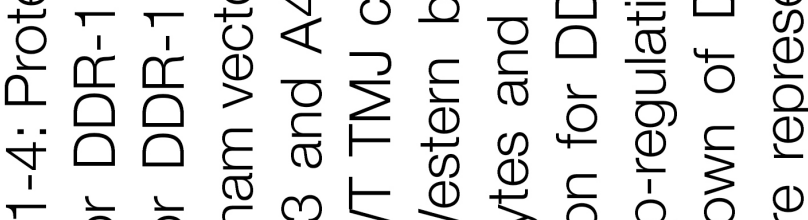

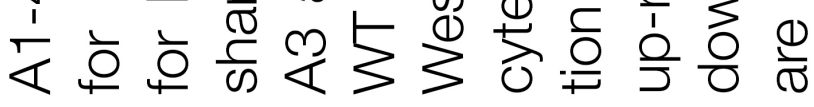
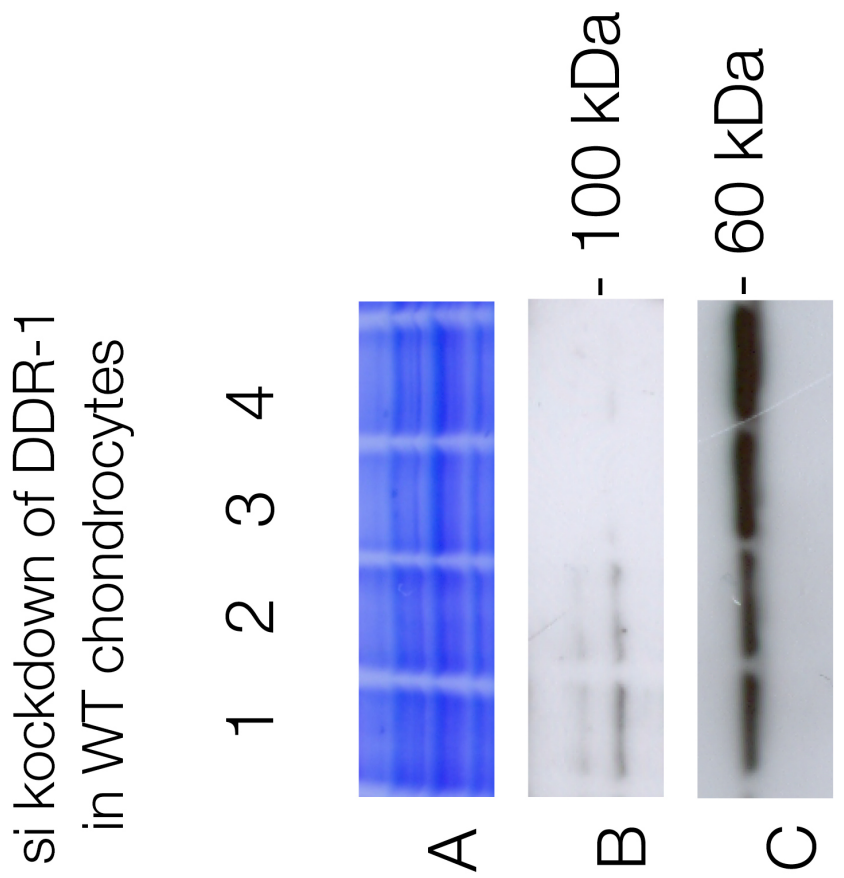


\section{Chapter 4}

Human migratory meniscus progenitor cells are controlled via the TGF $\beta$ pathway

Hayat Muhammad ${ }^{1 *}$, Boris Schminke ${ }^{1 *}$, Christa Bode ${ }^{1}$, Andrea Bernhard ${ }^{1}$, Moritz Roth ${ }^{1}$, Julius Albert ${ }^{1,}$ Silvia von der Heyde ${ }^{2}$, Vicki Rosen ${ }^{3}$ and Nicolai Miosge ${ }^{1 \#}$

${ }^{1}$ Tissue Regeneration Work Group, Department of Prosthodontics

Medical Faculty, Goerg August University

D-37075 Goettingen, Germany

${ }^{2}$ Institute of Medical Statistics, Medical Faculty,

Georg August University D-37075 Goettingen, Germany

${ }^{3}$ Developmental Biology, Harvard School of Dental Medicine,

188 Longwood Avenue, Boston, MA 02115, USA

\#Corresponding author

*Both authors contributed equally to this work

Hayat Muhammad's contribution: Progenitor cell isolations, protein isolations from tissue and cells, Western blots, PCR array, rt-RT-PCR, cell cultures also in the three dimensional system, paraffin embedding, optimization of the embedding protocol, cell induction experiments, gene silencing experiments, progenitor cell differentiation, in vitro and vivo migration experiments, protein overexpressions, proteomic data analyses, transcriptomics data analyses, immunohistochemistry and manuscript preparation. 
healthy mensicus
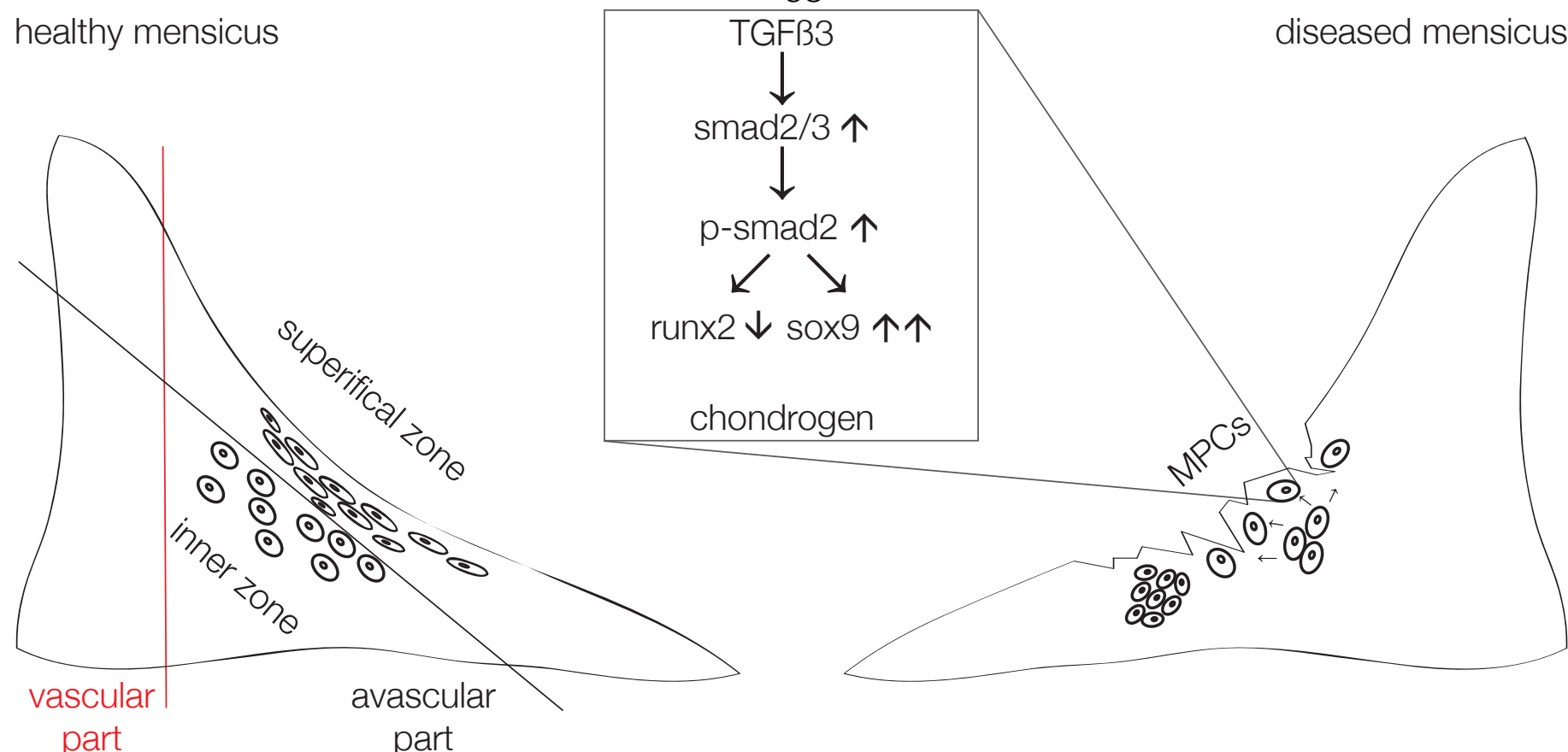

part

avascular

part

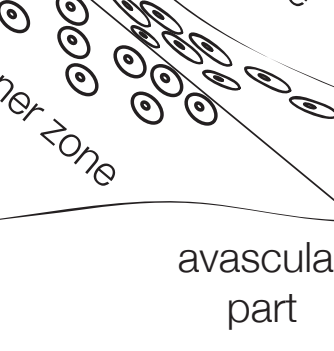


Highlights:

- Progenitor cells are found the inner avascular part of human osteoarthritic menisci.

- These cells (MPCs) are clonogenic, migratory and multipotent.

- MPCs are governed via the canonical TGF $\beta$-pathway.

-TGFß3 via smad2 reduces runx2 to enhance the chondrogenic potential of MPCs. 
Human migratory meniscus progenitor cells are controlled via the TGFß pathway

Hayat Muhammad ${ }^{1,4}$, Boris Schminke ${ }^{1,4}$, Christa Bode ${ }^{1}$, Moritz Roth ${ }^{1}$, Julius Albert ${ }^{1}$, Silvia von der Heyde 2 , Vicki Rosen ${ }^{3}$ and Nicolai Miosge ${ }^{1 \#}$

${ }^{1}$ Tissue Regeneration Work Group, Department of Prosthodontics, Medical Faculty, GeorgAugust-University, D-37075 Goettingen, Germany.

2Institute of Medical Statistics, Medical Faculty, Georg-August-University, D-37075 Goettingen, Germany.

${ }^{3}$ Developmental Biology, Harvard School of Dental Medicine, Boston, MA 02115, USA.

${ }^{4}$ Both authors contributed equally.

Corresponding author:

\# Tissue Regeneration Work Group, Department of Prosthodontics Medical Faculty, GeorgAugust-University; Robert Koch Straße 40, 37075 Goettingen, Germany, Tel.: 0049551393927, Fax.: 0049551398621, Email: nmiosge@gwdg.de

Keywords: human meniscus progenitor cells, osteoarthritis, TGFß pathway, regeneration 
Summary:

Degeneration of the knee joint during osteoarthritis often begins with meniscal lesions. Meniscectomy, previously performed extensively after meniscal injury, is now obsolete because of the inevitable osteoarthritis that occurs following this procedure. Clinically, meniscus self-renewal is well documented as long as the outer, vascularized meniscal ring remains intact. In contrast, regeneration of the inner, avascular meniscus does not occur. Here, we show that cartilage tissue harvested from the avascular inner human meniscus during the late stages of osteoarthritis harbors a unique progenitor cell population. These meniscus progenitor cells (MPC) are clonogenic, multipotent and exhibit migratory activity. We also determined that MPCs are likely to be controlled by canonical TGF $\beta$ signaling that leads to an increase in sox9 and a decrease in runx2, thereby enhancing the chondrogenic potential of MPC. Therefore, our work is relevant for the development of novel cell biological, regenerative therapies for meniscus repair. 
Introduction

The knee is particularly prone to meniscal lesions that lead to osteoarthritis (OA) (Englund et al., 2012), and a high interdependency of OA and meniscus lesions has been described (Brophy et al., 2012). Meniscal injuries are the most common knee injury and account for more than $50 \%$ of the 1.5 million knee arthroscopies performed annually (Englund et al., 2008; Lohmander et al., 2007). The prevalence of meniscal tears increases with age (Loeser, 2013) and may be as high as 56\% in men aged 70-90 years old (Englund et al., 2008). In the elderly, OA is the most common musculoskeletal disease (Reginster, 2002) and will be the fourth-leading cause of disability by the year 2020 (Woolf and Pfleger, 2003). OA often leads to painful loss of joint function, and current therapeutic interventions serve primarily as shortterm symptomatic relief. Although allografts or bio-engineered meniscal substitutes (Haddad et al., 2013; Steinert et al., 2007) fill the space void created following removal of the meniscus, clinical, radiological and $\mathrm{MRI}$ evaluations show no protection against the development of OA (Hommen et al., 2007). The specific reasons for this lack of effect are unknown; however, a failure to successfully remodel the allograft into living tissue is one likely factor (Steadman and Rodkey, 2005). Almost all patients eventually require joint replacement (Lohmander and Roos, 2007).

The meniscus is a fibrocartilage (Benjamin and Evans, 1990) comprising an outer, "red vascularized" part and an inner "white, unvascularized" part harboring round fibrochondrocytes (Hellio Le Graverand et al., 2001). As in hyaline cartilage tissue, the cells of the meniscus are also embedded in extracellular matrix composed of a framework of collagens together with proteoglycans and glycoproteins. The inner part exhibits a superficial zone with flattened, elongated fibroblast-like cells that predominantly synthesize collagen type I (McDevitt and Webber, 1990), whereas the round fibrochondrocytes from the inner part also 
produce collagen type II (Chevrier et al., 2009). Fibrochondrocytes have been isolated from bovine meniscus tissue, and their role in meniscus repair has been highlighted (Mauck et al., 2007). Reports have described cells migrating out of healthy adult rabbit meniscus (Webber et al., 1989). Others have harvested cells from human meniscus after collagenase digestion, that have been described as mesenchymal stem cells (Segawa et al., 2009). It is known that the peripheral vascular (red) zone of the human meniscus harbors CD34-positive cells (Osawa et al., 2013). Progress has been limited by a lack of information on meniscal cell progenitors and the signals controlling their proliferation and differentiation. Growth factors and fibrin clots have shown some promise and are consistent with the theory of an intrinsic healing potential of the meniscus via activation of endogenous meniscal stem cells (Petersen et al., 2005).

Here, for the first time, we describe human meniscus progenitor cells (MPC) isolated from late-stage OA meniscus samplesof the white, avascular part and show that their regenerative potential is governed by TGFB signaling. We believe that our results will help in the development of novel strategies for cell biological therapies for meniscus repair.

Results

Meniscus tissue histology and molecular composition

By combining and modifying available classification systems for OA specimens (Pauli et al., 2011; Zhang et al., 2011), we developed a means to discriminate healthier from diseased specimens in our study of human menisci (see Methods and Materials). Intact meniscus tissue is composed of a superficial zone with flattened cells that primarily synthesize collagen type I (McDevitt and Webber, 1990). This architecture remains in healthier human menisci in patients suffering from OA (Fig. 1A and B). The inner zone, containing more rounded cells 
that secrete collagen type I and type II, is also observed in the diseased specimens (Fig. 1C and F); however, the superficial zone is always lost in these samples. Moreover, in diseased human menisci, calcifications (Fig. 1D) and clusters of cells (Fig. 1E) are often found.

We then analyzed cells growing from the inner zone of diseased and healthier human menisci using microarrays. We focused on the 100 genes with the smallest $p$-values, and among these genes, we identified 48 as being up-regulated, i.e., exhibiting a positive fold change, and 52 as being down-regulated, i.e., exhibiting a negative fold change ( $p$-value $<0.001$, as listed in the figure legend). Interestingly, only 4 of the 15 potential marker genes (see Materials and Methods) were among these 100 genes, that is, TIMP2, SOX9, ACAN and MMP14, stressing the importance of these particular genes for discriminating healthier from diseased samples. When used to examine eight human meniscus tissue samples, the 100 top genes clustered into two groups, healthier and diseased, as shown in the heat map (Fig. 1G). A selection is listed (Fig. $1 \mathrm{H}$ ), and the complete results can be found under GEO (accession number: GSE52042). Furthermore, we subjected the cell lysates of cells from the patients to proteomic analyses (Fig. 1I). Approximately 4000 proteins were identified; however, only a small number are known to be relevant for OA and meniscus pathology (Fig. 1J). Interestingly smad2, a mediator of the canonical TGFß/activin signaling pathway was more abundant in healthier meniscus cells than in diseased cells. However, most of the remaining proteins were not different between healthier and diseased samples. Because there is no international convention or single platform for presenting the results from these profiling studies and the list is too long for a supplemental figure, they can be found online on our homepage (http:// www.miosge.med.uni-goettingen.de/de/?id=17). Taken together, our classification system and the results of the transcriptome and proteomic analyses enable the unequivocal discrimination of healthier and diseased human meniscus specimens. 
TGFß, smads, runx2 and sox9 in human osteoarthritic meniscus tissue

Because the TGFB family of proteins has been described to be important for meniscus pathology and we observed changes in smad2 levels that varied with disease severity, we performed immunohistochemistry to examine the TGF $\beta$ status in our samples. We observed greater staining for TGFB3 in healthier (Fig. 2A) tissue compared with diseased (Fig. 2B) human menisci and greater smad2/3 staining in healthier tissue (Fig. 2D) compared with diseased menisci (Fig. 2E). In addition, reduced staining for smad1 was observed in (Fig. 2C) diseased tissue compared with healthier specimens (data not shown), whereas smad4 appeared to be similar between the 2 groups of human menisci (Fig. 2F and data not shown). These immunohistochemical findings were verified by Western blotting using tissue extracts from the inner zone of the menisci. All blots were quantified using ImageJ64 with a-Tubulin used as a control (Fig. 2G). A significantly higher level of smad2/3 was detected in healthier than in diseased tissue extracts (Fig. 2H). Interestingly, this finding correlated with the greater level of runx2 in diseased specimens (Fig. 2l), whereas sox9 levels were not altered significantly (Fig. 2J). However, smad4 was increased in the diseased menisci (Fig. 2K). Real time RT-PCR revealed results consistent with Western blotting for the expression of TGFB3 (Fig. 2L), runx2 (Fig. 2M) and sox9 (Fig. 2N) mRNA. The expression of collagen type II mRNA was reduced (Fig. 20) in line with the sox9 mRNA. Therefore, a reduction in TGFB3 and smad2/3 in diseased meniscus is found together with greater levels of runx2. This pattern of chondro- and osteogenic factors has been identified as a hallmark of osteochondroprogenitor cells that are present in human tissue at late stages of OA (Koelling et al., 2009; Zhang et al., 2011). This finding guided us to investigate further the nature of the cells from diseased human meniscus tissue of the inner zone in vitro. 
Cells from diseased human meniscus in vitro are similar to the cells found in vivo

Explant cultures of diseased human meniscus from late-stage OA revealed the migration of cells outwards after 7 to 10 days (Fig. 3A). This finding was not observed in healthier specimens containing a superficial zone. After collagenase digestion of the inner zone, more round cells were observed in P1 (Fig. 3B) that were positive for collagen type I (Fig. 3D). However, the cells obtained from the superficial zone of healthier menisci in P1 were more elongated (Fig. 3C) and were also positive for collagen type I (Fig. 3E). Because most of the OA cases in medicine are identified at late disease stages when the superficial zone of the meniscus has been completely lost, we concentrated on the cells from the inner, "white", avascular zone. Real time RT-PCR of the cells of the inner zone revealed significantly higher levels of mRNA for TGFB3 (Fig. 3 F), smad2 (Fig. 3G) and smad3 (Fig. 3H) in the cells derived from menisci with a lower disease score (healthier menisci), whereas sox9 was generally unchanged (Fig. 3l), which is consistent with results from meniscus samples in vivo (Fig. 2). When examined by immunocytochemistry, smad1 para-nuclear staining (Fig. 3J), smad2/3 (Fig. 3K) and smad4 (Fig. 3L) were all detected in cells from diseased menisci. A TGFB/BMP pathway PCR array confirmed the involvement of this pathway in meniscal disease, as shown here by significantly higher levels of mRNA for smad7 (Fig. 3M), noggin (Fig. 3N) and follistatin (Fig. 30) in meniscus cells from diseased tissues. 
The cells from the inner zone of the diseased meniscus show progenitor cell characteristics and a high migration potential

In culture, the cells derived from human meniscal tissue grow clonally and exhibit a spindle shaped phenotype typical of mesenchymal stem cells. A FACS analysis for the stem cell markers stro1, CD29, CD105 and CD106 on cells migrating out of the inner zone of diseased menisci showed significantly higher levels of these antigens than in cells released after collagenase digestion of healthier specimens where the superficial zone remained intact (Fig. 4A). Both populations were negative for the hematopoietic markers CD45 and CD34 (data not shown). The cells were driven towards the chondrogenic lineage in a 3D alginate culture (3D) and with the help of standard chondrocyte differentiation medium (3D + chond. diff.). We used a-Tubulin (Fig. 4A) for quantification purposes with the ImageJ64 program. The cells from damaged menisci showed $60 \%$ more sox9 (Fig. 4C), whereas runx2 was no longer detected (Fig. 4D). Concomitant with the pattern of these transcription factors, collagen type II appeared in the 3D and 3D + chondrogenic differentiation culture medium; however, it was undetectable in undifferentiated cells in 2D culture (Fig. 4E). The levels of sox9 (Fig. 4F) and collagen type II mRNA (Fig. 4G) were also significantly increased when cells were grown in 3D and chondrocyte differentiation medium. In contrast, the levels of collagen type I mRNA decreased (Fig. 4H). Following osteogenic differentiation, a proportion of cells became positive for alkaline phosphatase (Fig. 4I) and osteocalcin (Fig. 4J), as well as Alizarin red (data not shown). Oil red-positive (Fig. 4K) and PPARy positive (data not shown) adipocytes were identified following adipogenic differentiation.

To examine the cell migration potential ex vivo, (Fig. 5A), a sample of diseased meniscus tissue was placed on the scraped side of a tissue culture flask that had been $80 \%$ confluent with GFP-labeled primary meniscus cells. After 3 days, green cells were found inside the 
tissue sample (Fig. 5B), which was confirmed by placing the sample in a new flask (Fig. 5C). After an additional 7-10 days, cells, both green and unlabeled, residing in the damaged meniscus sample were again found outside the tissue. Cells migrated only into diseased meniscus tissues and not into the ones containing a intact superficial zone. Fixed samples allowed the identification of GFP-labeled meniscus-derived cells in the tissue sample (data not shown). Furthermore, a standard Boyden chamber assay revealed that the meniscusderived cells migrated more significantly toward a gradient of human recombinant PDGF (Fig. 5D), as already shown for chondrogenic progenitor cells (Koelling et al., 2009). Taken together, the multi-lineage differentiation potential and the migration results enabled us to name the cells 'meniscus progenitor cells' (MPCs). 
The influence of TGFB3, runx2 and smads on the chondrogenic potential of MPCs

MPCs were treated with $10 \mathrm{ng} / \mathrm{ml}$ TGFB3 in vitro. a-Tubulin was used as loading control and for the quantification (Fig. 6A). MPCs exposed to TGFB3 exhibited significantly greater levels of smad2/3 (Fig. 6B) and also significantly greater levels of p-smad2 (Fig. 6C). Treatment with TGFB3 also increased the levels of sox9 in the MPCs compared with controls (Fig. 6D) while also decreasing runx2 levels significantly (Fig. 6E). MPCs treated with $10 \mathrm{ng} / \mathrm{ml}$ BMP2 showed significantly reduced mRNA levels of smad2 (Fig. 6F) and sox9 (Fig. 6G).

We performed a runx2 knock-down on MPCs. Equal loading is indicated by a-Tubulin (Fig. 7A). When runx2 was knocked down in MPCs by siRNA interference (Fig. 7B), smad2/3 levels increased (Fig. 7C); more importantly, p-smad2 became detectable only after the runx2 knock-down (Fig. 7D). In summary, we observed that TGFB3 treatment increased sox9 and decreased runx2 in MPCs, thereby enhancing their chondrogenic potential.

The influence of smad1 and smad2 was evaluated via overexpression. Again a-Tubulin served for quantification purposes (Fig. 7E). Smad1 overexpression was confirmed (Fig. 7F), as was that of smad2 (Fig. 7G). The overexpression of smad1, as well as smad2 in MPCs increased sox9 significantly (Fig. $7 \mathrm{H}$ ). In contrast, smad2 overexpression enhanced runx2 levels more significantly then smad1 overexpression (Fig. 7l). These results demonstrate that the chondrogenic potential of MPCs is likely to be controlled by TGFB3-mediated phosphorylation of smad2, which in turn up-regulates sox9 and down-regulates runx2. 


\section{Discussion}

The menisci harvested from patients undergoing total knee replacement showed typical pathological signs described for osteoarthritic articular cartilage (Loeser et al., 2012), including the presence of calcifications, areas of fatty degeneration and chondrocyte cluster formation (Hellio Le Graverand et al., 2001). The simple histopathological grading system we applied to our samples is based on these disease signs, and when combined with the absence or presence of the meniscal superficial zone, it enabled the separation of the samples of early $\mathrm{OA}$ (healthier specimens) from those that had progressed to severe degenerative disease (diseased specimens). The discriminative power of this grading system was mirrored in our transcriptome analysis, where the molecular pattern found in diseased samples was distinct from that found in early stage OA tissues. This was already shown for menisci of different age (Rai et al., 2013). Meniscus samples that received a high disease score also showed reduced immunohistochemical staining for TGFB and smad2. Consistent with these findings, proteome analysis and Western blotting of diseased specimens also showed a reduction in smad2 protein and an up-regulation of runx2 compared with healthier specimens. These results, together with the literature cited indicated that the TGFB/BMP pathway, with its dual osteogenic and chondrogenic actions (Massague, 2012), was a good candidate to investigate in greater detail in vitro.

Previous results (Koelling et al., 2009; Seol et al., 2012) indicated the existence of progenitor cells in cartilage tissues from late stage OA. Therefore, we performed an explant culture from diseased meniscus tissue and found cells migrating out of these specimens after a few days, which was not observed for healthier menisci. The cells maintained their round phenotype at least in P1 in cell culture and were positive for collagen type I, as described previously (Chevrier et al., 2009; Hellio Le Graverand et al., 2001). In contrast, the cells from the 
superficial zone of healthier specimens after collagenase digestion exhibited the elongated spindle-shaped phenotype of fibroblasts and were also positive for collagen type I (McDevitt and Webber, 1990). These meniscal-derived cells expressed smad2 and smad3 as determined by quantitative real time RT-PCR and immunocytochemistry and also exhibited an up-regulation of noggin, a BMP signaling antagonist, and smad7, a BMP/TGF $\beta$ signaling antagonist, when examined using a commercial PCR array. A FACS analysis of these migratory meniscal cells showed that they were positive for well-known stem cell markers including stro-1, CD105 or CD106. Quite remarkably, we found that these meniscal cells were capable of migrating into diseased meniscus tissue and re-populating it and that they also migrated toward a PDGF gradient in a Boyden chamber assay. We also determined that these highly migratory meniscal-derived cells were multipotent; they synthesized collagen type II and suppressed runx2 in 3D culture when grown in chondrogenic medium and became positive for markers of the osteoblastic lineage or adipogenic lineage after exposure to appropriate differentiation media. Interestingly, a significant difference in marker expression was observed when we compared cells migrating out of diseased meniscal explants with cells derived from meniscal tissue digestions, which indicates that the migratory progenitor cells are a subpopulation of the cells found in diseased human meniscus tissue in vivo. When the protein expression characteristics of the mesenchymal stem cell markers are considered together with their clonicity, multipotency and migratory potential, we name these cells meniscus progenitor cells (MPCs). The cells show signs of senescence (data not shown); therefore, we prefer the term progenitor rather than stem cell. The MPCs are different from the recently described CPCs (Koelling et al., 2009; Koelling and Miosge, 2010) not only due to their tissue origin and stem cell marker pattern, but also due to their ability to produce collagen type I and type II. However, they are similar as they also appear to be modulated via 
a balance between runx2 and sox9 (Muhammad et al., 2013).

In articular cartilage, deletion of the type 2 TGF $\beta$ receptor results in the up-regulation of runx2, MMP13 and ADAMTS5, which correlates with the progression of OA disease (Shen et al., 2013), and the stimulation of smad2/3 signaling has been suggested as a potential therapeutic manipulation for articular chondrocytes for the treatment of OA (van der Kraan et al., 2012). Here, we demonstrate, that the treatment of MPCs with TGFB3 results in an upregulation of sox9 and a down-regulation of runx2. We also show that knock-down of runx2 in MPCs enhanced total smad2/3 and $\mathrm{p}$-smad2, data that underline the positive chondrogenic effect of TGFB3, which appears to be mediated via p-smad2, on MPCs. In contrast, direct BMP2 stimulation of MPCs reduced smad2 levels, enabling the suggestion that the influence of the TGFB/smad2 axis is more prominent in MPCs, which allows chondrogenesis. The shift in articular chondrocytes from smads $2 / 3$ to smads $1 / 5$ appears to be important for the development of $\mathrm{OA}$, and recently, a balancing role of endoglin (CD 105) for this shift has been highlighted (Finnson et al., 2010). This may also be the case in the diseased human meniscus, as we found lower levels of smad2/3 and higher levels of runx2 in diseased tissue compared with healthier meniscus tissue. The complex regulatory role of the signaling balance between TGFB/BMP in OA pathology is widely acknowledged (Finnson et al., 2010; van der Kraan et al., 2010), and our data suggest this interaction also governs meniscal cell behavior during the development and progression of OA. Furthermore, our findings suggest that specific players of the TGFB pathway may be suitable targets for regenerative therapies directed at diseased human meniscus in late stages of OA. Because some studies support the notion that direct TGFB treatment of joint structures has undesirable side effects, including synovial hyperplasia, inflammation or even osteophyte formation (Blaney Davidson et al., 2006; van Beuningen et al., 1998), in the future, a more 
nuanced understanding of the regulatory events governing MPC biology will be necessary to identify a cell-based therapy for the regeneration of diseased meniscus tissue.

\section{Experimental Procedures}

Tissues and preparation

Adult osteoarthritic lateral and medial menisci were obtained from more than 150 patients (ages 62-75 years) suffering from late-stage OA after total knee replacement operations. The patients met the American College of Rheumatology classification criteria and provided their written informed consent. Histopathological classification of OA cartilage confirmed the presence of late-stage OA (Pritzker et al., 2006) of the tibial plateau and femoral condyle in all samples.

Histology and meniscus grading

For light microscopy, meniscus specimens $(\mathrm{n}=80)$, including the white and red areas (Fig. 1A, inset), were processed as described previously (Koelling and Miosge, 2010) and a combined Alcian blue/nuclear fast-red staining was performed. Based on existing grading systems (Pauli et al., 2011; Zhang et al., 2011), we developed a simple score for meniscus degradation. The presence ( 1 point) or absence ( 2 points) of the superficial zone and the intensity of the Alcian blue staining (high $=1$ point or low $=2$ points) were used for evaluations. The presence of fatty degeneration and/or cell clusters (2 points) or the presence of calcifications (3 points) was also included. A minimum of 2 and a maximum score of 9 points can be reached. The threshold was set to 4 points. Three independent histologists evaluated more than 40 samples and identified 31 with a score of above 4 (diseased) and 16 with scores of below 4 (healthier). The remaining samples were not evaluated unequivocally. 
Cell isolation and culture

Tissue pieces measuring 7-10 $\mathrm{mm}^{3}$ from the central inner zone (white area) of the healthier $(n=12)$ and diseased $(n=12)$ human menisci were excised, and care was taken not to include the outer red zone. Samples of healthier menisci with an intact superficial zone (grading score: 2, $\mathrm{n}=12$ ) were also excised. After 7-10 days of incubation, only samples that lacked a superficial zone showed outgrowth of cells, which were harvested, and $10^{3} \mathrm{cells} / \mathrm{cm}^{2}$ were transferred to a monolayer culture under standard conditions in Dulbecco's modified Eagle's medium (DMEM) containing 10\% fetal bovine serum (GIBCO, lot number 41F2061K). 3Dimentional culturing was performed using alginate beads. Samples of $2-3 \mathrm{~mm}^{3}$ in size from the superficial area of healthier menisci $(n=7)$ and the central area of diseased menisci $(n=7)$ were harvested and digested with collagenase I (152 U/ml; Invitrogen, Karlsruhe, Germany), collagenase II (280 U/ml; Biochrom, Berlin, Germany), and dispase (15 U/ml; Invitrogen) for 6 $\mathrm{h}$ at $37^{\circ} \mathrm{C}$. To exclude cell-matrix residues, we filtered the collagenase-treated cells through a 40- $\mu \mathrm{m}$ mesh sieve (BD Falcon, Heidelberg, Germany).

Microarray analysis and bioinformatic methods

Quality control and the quantification of total RNA samples was performed, and data from 10 human samples was measured using the microarray (Microarrays Inc., Huntsville, USA) ReadyArray (HS1100), which contains 48,958 probes per microarray slide from the Stanford/ Illumina collaboration on the HEEBO (Human Exonic Evidence Based Oligonucleotide) set of long oligos. This chip was used in a 1-color assay with a reference measurement of internal expression signals from several housekeeping genes. An internal QC was run to ensure these signals were within expected ranges for the array. First, the data were curated, primarily from non-zero flag values that indicate absent or poor-quality spots, resulting in 13,585 probes. 
Next, quantile-normalization and logarithmizing followed. We then selected 15 potential marker genes, TIMP1, TIMP2, TIMP3, COL3A1, RUNX2, SOX9, ACAN, PRG4, DCN, MMP9, MMP3, MMP14, ADAMTS20, ADAMTS1 and NID2, to determine whether each of the 10 samples belonged to the 'diseased' or 'healthier' group. A Pearson correlation-based hierarchical clustering approach including row-scaling and Ward's minimum variance method was chosen. This analysis revealed two groups of four samples each. For the selected eight samples, a differential expression analysis including all 13,585 probes with an empirical Bayes statistic of the limma package, moderated gene-by-gene t-tests and p-value adjustments via the Benjamini-Hochberg method was performed. Data were submitted to GEO (accession number: GSE52042).

Proteome analysis

For in-gel digestion, gel bands were washed with water, reduced with dithiothreitol (10 mM in $100 \mathrm{mM} \mathrm{NH}_{4} \mathrm{HCO}_{3}, 50 \mathrm{~min}, 56^{\circ} \mathrm{C}$ ) and alkylated with $55 \mathrm{mM}$ iodoacetamide. Gel spots were digested twice overnight at $37^{\circ} \mathrm{C}$ with porcine trypsin $\left(12.5 \mathrm{ng} / \mu \mathrm{l}\right.$ in $50 \mathrm{mM} \mathrm{NH} \mathrm{HCO}_{3}$ and 5 $\mathrm{mM} \mathrm{CaCl}_{2}$ ). Peptide extraction was performed using aqueous acetonitrile, and mass spectrometric analysis was performed following concentration of the samples using a reversed phase-C18 precolumn $(0.15 \mathrm{~mm}$ ID $\times 20 \mathrm{~mm}$ self-packed with Reprosil-Pur120 C18-AQ 3 um material) and separation using reversed phase-C18 nanoflow chromatography (New Objective, Woburn, USA). The eluent was analyzed using a Top10 method in the Data Dependent Acquisition mode on the $\mathrm{Q}$ Exactive high-resolution mass spectrometry system (Thermo Scientific, Dreieich, Germany) operated under Tune 2.2 using HCD fragmentation, with normalized collision energy of $25 \%$. Peak lists were generated using the Raw2MSM v1.10 software (MPI for Biochemistry, Martinsried, Germany). All MS/MS samples were 
analyzed using Mascot (Matrix Science, London, UK; version 2.4.1) set to search the NCBInr_20130805 database (selected for Homo sapiens, 251,430 entries). Further details, especially about normalization procedures, have already been published (Christian et al., 2013).

\section{Antibodies}

Monoclonal anti-type II collagen (CIIC1) and anti-Stro1 antibodies were obtained from the Developmental Studies Hybridoma Bank, University of lowa, USA. For Western blotting, monoclonal a-Tubulin antibody (Sigma-Aldrich, Saint Louis, USA) was used as a loading control. Monoclonal anti-smad2/3 (c-8, sc-133098), anti-smad1 (A-4, sc-7965), anti-sox9 (H-90, sc-2095), anti-runx2 (M-70, sc10758) and polyclonal anti-smad4 (c-20, sc-19099) antibodies were purchased from Santa Cruz Biotechnology, Heidelberg, Germany. Polyclonal anti-phospho-smad2 (Ser465/467), and anti-TGF33 antibodies were purchased from Cell Signaling Technology Inc., Frankfurt am Main, Germany. We used anti-human-PE/FITC monoclonal immunoglobulin isotype controls (BD PharMingen, Mountain View, USA) or fluorescein isothiocyanate (FITC)-coupled CD29, CD34, CD45, CD90, CD106 (Miltenyi Biotec, Bergisch Gladbach, Germany) and CD105 (Alexa flour 488) primary antibodies (Biolegend, London, UK). Goat anti-mouse, goat anti-rabbit-FITC IgG (Dianova, Hamburg, Germany) and anti-Turbo-GFP (Evrogen, Heidelberg, Germany) secondary antibodies were used for immunocytochemistry.

Immunohistochemistry

After the histopathological grading, 10 diseased and 10 healthier samples were processed for 
immunohistochemistry as described elsewhere (Koelling and Miosge, 2010). The HiDefDetection ${ }^{R}$ alkaline phosphatase mouse/rabbit system (Cell Marque, Rocklin, CA, USA) was used. Immunoreactions were also performed with swine serum as a negative control, and figures show representatives of three individual experiments.

Immunoblotting

Total protein extraction from tissues and cells was performed using $5 \mathrm{M}$ guanidine hydrochloride and protease inhibitors. Pellets were precipitated in ethanol, washed in PBS, precipitated again and finally dissolved in PBS containing 0.4\% SDS. SDS-PAGE was performed using $6 \%$ acrylamide in the stacking gel and $10 \%$ in the separation gel. Protein detection was performed as described previously (Koelling et al., 2009) and quantified using a-Tubulin as a loading control using the ImageJ64 program.

RNA extraction and cDNA synthesis

Cells from samples of healthier and diseased menisci in P1 monolayer cultures were lysed directly in RLT buffer and subjected to RNA isolation, according to the manufacturer's instructions (RNeasy Mini Kit, Qiagen, Hilden, Germany). The alginate beads from the 3D cell cultures were first dissolved using EDTA buffer. The QuantiTect Reverse Transcription Kit (Qiagen, Hilden, Germany) was used.

Quantitative rtRT-PCR

A $10 \mu$ volume per PCR reaction comprising $1 \mathrm{ng}$ of cDNA, $5 \mu \mathrm{l}$ of Platinum Sybr Green qPCR SuperMix ${ }^{\mathrm{TM}}$ (Invitrogen, Darmstadt, Germany) and 20 pmol of each primer was 
chosen. The program primer 3 (http://frodo.wi.mit.edu/cgibin/primer3) was used to design the primers:

\begin{tabular}{lccr}
\hline \multicolumn{1}{c}{ GenelD } & Forward primer & Reverse primer & Accession no. \\
\hline Smad1 & tcttcagagccaccatgaactaa & aaccagcacaggaggaagtacag & NM_005900 \\
Smad2 & gtctcttgatggtcgtctcca & ttctgttaggatctcggtgtgtc & NG_029946 \\
Smad3 & ccatcctgcctttcactc & tggtgatgcacttggtgt & AB004922 \\
Smad4 & gcacaaggttggttgctaaga & gcagaacagtgagacattaggtagag & NG_013013 \\
Tgfb3 & ctttggacaccaattactgcttc & gggttcagagtgttgtacagtcc & NM_003239.2 \\
Runx2 & ttccagaccagcagcactc & cagcgtcaacaccatcatt & NM_004348 \\
Col1(a1) & ttccccagccacaaagagtc & cgtcatcgcacaacacct & NM_000088 \\
Col2(a1) & ctcctggagcatctggagac & accacgatcacccttgactc & NM_033150 \\
Sox9 & caggctttgcgatttaagga & ccgtttaaggctcaaggtg & Z46629 \\
B2M & tgctgtctccatgtttgatgtatct & tctctgctccccacctctaa & NM_004048 \\
\hline
\end{tabular}

The PCR program was set for 40 cycles: initial activation for 3 min at $95^{\circ} \mathrm{C}$, denaturation at $95^{\circ} \mathrm{C}$ for $20 \mathrm{~s}$, appropriate annealing temperature for $20 \mathrm{~s}$, elongation at $72^{\circ} \mathrm{C}$ for $20 \mathrm{~s}$ and a melting curve in $0.1^{\circ} \mathrm{C}$ steps from $50-95^{\circ} \mathrm{C}$ with the aid of the Mastercycler Realplex2 $\mathrm{S}^{\circledR}$ (Eppendorf, Hamburg, Germany). Data were normalized to a highly consistent housekeeping gene ( $\beta 2 \mathrm{M})$. PCR products were sequenced (Seqlab, Goettingen, Germany) to confirm the product identity. The relative ratios were calculated (Pfaffl, 2001). Every PCR was run three times in triplicate. The intra-test and inter-test variations were $<1 \%$. Primer efficiencies ranged from 1.9-2.1.

Cell differentiation

Chondrogenic differentiation was performed using 3D cell culture. When the cells reached 70\% confluency, MPCs from P1 monolayer cultures were subjected to 3D culture. Cells $(40,000)$ were encapsulated in low-viscosity alginate and standard chondrogenic medium. For osteogenic differentiation, a total of $1000 \mathrm{MPCs} / \mathrm{cm}^{2}$ in $75 \mathrm{~cm}^{2}$ flasks were differentiated 
into cells of the osteoblastic lineage under the influence of $\mathrm{NH}$ OsteoDiffMedium or adipogenic differentiation was performed using NH AdipoDiffMedium (Miltenyi Biotec, Bergisch Gladbach, Germany).

Immunofluorescence microscopy

P1 cells were grown on coverslips, fixed in $70 \%$ ethanol and incubated with $100 \mu$ of primary antibody (1:50 dilution in PBS) for $1 \mathrm{~h}$ at RT. In the case of uncoupled primary antibodies, we then added secondary fluorescence-coupled antibodies (1:500 dilution in PBS) for 20 min at RT. Cells were than washed twice and finally stained with DAPI. A fluorescence microscope was used for cell examination, and a Nikon D90 camera (Nikon, Duesseldorf, Germany) was used to take all images.

\section{FACS analysis}

To test cells for stem cell markers, $10^{6}$ cells were suspended in $100 \mu$ l of PBS containing $1 \mu$ l of fluorescence-coupled antibody at RT for $1 \mathrm{~h}$ in the dark. The cells were then washed twice and centrifuged at $800 \mathrm{rpm}$ for $10 \mathrm{~min}$. Finally, the cells were subjected to FACS analysis using a FACScan machine (Becton Dickinson, Mountain View, CA, USA), and 10,000 living cells were analyzed (Koelling et al., 2009). For data evaluation, we used the WinMDlv2.9 program (Scripps Research Institute, La Jolla, USA). The FACS Vantage SE (Becton Dickinson, Mountain View, USA) was applied for cell selection and the Cell Quest Pro 2000 software package was used for analysis. 
The human TGFB/BMP signaling pathway array was used (PAHS-035ZA_0123, SAbiosciences, Hilden, Germany), according to the manufacturer's instructions, and data were normalized using the standard program of the company's online platform.

GFP transfection

The cells $\left(5 \times 10^{5}\right)$ were transfected with $2.5 \mu \mathrm{g}$ of the pmaxGFP vector (Lonza, Koeln, Germany) in $100 \mu \mathrm{l}$ of nucleofector solution using the Amaxa program U-23. After transfection, the cells were transferred to DMEM supplemented with 20\% FCS, incubated overnight, and then cultured under standard conditions. The transfection efficiency reached approximately $80 \%$. FACS further enriched GFP-positive cells to approximately $97 \%$.

Migration and integration assay

In an ex vivo assay, a sample of diseased meniscus tissue was placed on the scraped side of a flask that had been $80 \%$ confluent with GFP-labeled cells. After 5-7 days, the specimens were washed with PBS, transferred to a new flask and examined using a fluorescence microscope. For the in vitro migration assay, we used a commercial two-chamber system with filters featuring 8-nm pores (CytoSelect, Cell Biolabs, San Diego, USA) and extinctions were measured at $560 \mathrm{~nm}$. Human recombinant PDGF (R\&D Systems, Abingdon, UK, $10 \mathrm{ng} /$ $\mathrm{ml}$ was applied as chemoattractant.

Cell induction experiment

Cells in 3D alginate were stimulated using recombinant human BMP2 $(10 \mathrm{ng} / \mathrm{ml}$, lot\# MSA3612112) and TGFB3 (10 ng/ml, lot\# MSA3612112), both of which were obtained from 
R\&D Systems, Abingdon, UK. After 24 hrs, the cells were harvested and subjected to total protein and RNA extraction.

siRNA transfections

The iLenti-GFP siRNA expression vector (ABM, Crestwood, Canada) was used for runx2 knock-down. The probe (CAGCACGCTATTAAATCCAAATT) that targeted runx2 was placed under the control of $\mathrm{H} 1 / \mathrm{H} 6$ and the GFP sequence under the CMV promoter. Control experiments were performed using a vector containing a scrambled siRNA sequence or without any vector. For the transfection of cells, see above.

\section{Overexpression}

Smad1 and smad2 cloned into the pCMV5-Flag and pCMV5B-HA vectors were purchased from Addgene, Cambridge, USA. The cells were transfected either with the expression vector or the vector without the insert, as described above.

Statistical analyses

Statistical product and service solutions (SPSS, Chicago, USA) software version 13.0 was used. The observed data were tested statistically, and the representative data shown are the means and standard deviation of at least three independent experiments, if not stated otherwise. After testing for normality of distribution and homogeneity of variances, we performed ANOVAs and post-hoc pairwise comparisons of the mean values. The Pearson correlation coefficients were calculated to examine the relationships between parameters. A $p$-value $<0.05$ was considered significant. 


\section{Acknowledgements}

The authors would like to thank Prof. H. Urlaub and Dr. C. Lenz from the joint proteome facility at the MPI for Biophysical Chemistry, Goettingen and the Medical Faculty of the University of Goettingen. We would also like to thank Dr. K. Jung from the Institute of Medical Statistics, Medical Faculty Goettingen.

Authors' contributions

Hayat Muhammad: Study conception, study design, acquisition of data, analyses and interpretation of data, manuscript preparation, and statistical analyses.

Boris Schminke: Study conception, study design, acquisition of data, analyses and interpretation of data, manuscript and figure preparation, and statistical analyses.

Christa Bode: Acquisition of data.

Moritz Roth: Acquisition of data.

Julius Albert: Acquisition of data.

Silvia von der Heyde: Microarray analysis and bioinformatic methods.

Vicki Rosen: Study conception, study design, interpretation of data, and manuscript preparation.

Nicolai Miosge: Study conception, study design, interpretation of data, and manuscript preparation. 


\section{References}

Benjamin, M., and Evans, E.J. (1990). Fibrocartilage. Journal of anatomy 171, 1-15.

Blaney Davidson, E.N., Vitters, E.L., van der Kraan, P.M., and van den Berg, W.B. (2006). Expression of transforming growth factor-beta (TGFbeta) and the TGFbeta signalling molecule SMAD-2P in spontaneous and instability-induced osteoarthritis: role in cartilage degradation, chondrogenesis and osteophyte formation. Annals of the rheumatic diseases $65,1414-1421$.

Brophy, R.H., Rai, M.F., Zhang, Z., Torgomyan, A., and Sandell, L.J. (2012). Molecular analysis of age and sex-related gene expression in meniscal tears with and without a concomitant anterior cruciate ligament tear. The Journal of bone and joint surgery American volume 94, 385-393.

Chevrier, A., Nelea, M., Hurtig, M.B., Hoemann, C.D., and Buschmann, M.D. (2009). Meniscus structure in human, sheep, and rabbit for animal models of meniscus repair. Journal of orthopaedic research : official publication of the Orthopaedic Research Society 27, 1197-1203.

Christian, H., Hofele, R.V., Urlaub, H., and Ficner, R. (2013). Insights into the activation of the helicase Prp43 by biochemical studies and structural mass spectrometry. Nucleic acids research.

Englund, M., Guermazi, A., Gale, D., Hunter, D.J., Aliabadi, P., Clancy, M., and Felson, D.T. (2008). Incidental meniscal findings on knee MRI in middle-aged and elderly persons. The New England journal of medicine 359, 1108-1115.

Englund, M., Roemer, F.W., Hayashi, D., Crema, M.D., and Guermazi, A. (2012). Meniscus pathology, osteoarthritis and the treatment controversy. Nature reviews Rheumatology 8, 412-419. 
Finnson, K.W., Parker, W.L., Chi, Y., Hoemann, C.D., Goldring, M.B., Antoniou, J., and Philip, A. (2010). Endoglin differentially regulates TGF-beta-induced Smad2/3 and Smad1/5 signalling and its expression correlates with extracellular matrix production and cellular differentiation state in human chondrocytes. Osteoarthritis and cartilage / OARS, Osteoarthritis Research Society 18, 1518-1527.

Haddad, B., Pakravan, A.H., Konan, S., Adesida, A., and Khan, W. (2013). A systematic review of tissue engineered meniscus: cell-based preclinical models. Current stem cell research \& therapy $8,222-231$.

Hellio Le Graverand, M.P., Vignon, E., Otterness, I.G., and Hart, D.A. (2001). Early changes in lapine menisci during osteoarthritis development: Part I: cellular and matrix alterations. Osteoarthritis and cartilage / OARS, Osteoarthritis Research Society 9, 56-64.

Hommen, J.P., Applegate, G.R., and Del Pizzo, W. (2007). Meniscus allograft transplantation: ten-year results of cryopreserved allografts. Arthroscopy : the journal of arthroscopic \& related surgery : official publication of the Arthroscopy Association of North America and the International Arthroscopy Association 23, 388-393.

Koelling, S., Kruegel, J., Irmer, M., Path, J.R., Sadowski, B., Miro, X., and Miosge, N. (2009). Migratory chondrogenic progenitor cells from repair tissue during the later stages of human osteoarthritis. Cell stem cell 4, 324-335.

Koelling, S., and Miosge, N. (2010). Sex differences of chondrogenic progenitor cells in late stages of osteoarthritis. Arthritis and rheumatism 62, 1077-1087.

Loeser, R.F. (2013). Aging processes and the development of osteoarthritis. Current opinion in rheumatology 25, 108-113.

Loeser, R.F., Goldring, S.R., Scanzello, C.R., and Goldring, M.B. (2012). Osteoarthritis: a disease of the joint as an organ. Arthritis and rheumatism 64, 1697-1707. 
Lohmander, L.S., Englund, P.M., Dahl, L.L., and Roos, E.M. (2007). The long-term consequence of anterior cruciate ligament and meniscus injuries: osteoarthritis. The American journal of sports medicine 35, 1756-1769.

Lohmander, L.S., and Roos, E.M. (2007). Clinical update: treating osteoarthritis. Lancet 370, 2082-2084.

Massague, J. (2012). TGFbeta signalling in context. Nature reviews Molecular cell biology 13, 616-630.

Mauck, R.L., Martinez-Diaz, G.J., Yuan, X., and Tuan, R.S. (2007). Regional multilineage differentiation potential of meniscal fibrochondrocytes: implications for meniscus repair. Anatomical record 290, 48-58.

McDevitt, C.A., and Webber, R.J. (1990). The ultrastructure and biochemistry of meniscal cartilage. Clinical orthopaedics and related research, 8-18.

Muhammad, H., Schminke, B., and Miosge, N. (2013). Current concepts in stem cell therapy for articular cartilage repair. Expert opinion on biological therapy 13, 541-548.

Osawa, A., Harner, C.D., Gharaibeh, B., Matsumoto, T., Mifune, Y., Kopf, S., Ingham, S.J., Schreiber, V., Usas, A., and Huard, J. (2013). The use of blood vessel-derived stem cells for meniscal regeneration and repair. Medicine and science in sports and exercise $45,813-823$.

Pauli, C., Grogan, S.P., Patil, S., Otsuki, S., Hasegawa, A., Koziol, J., Lotz, M.K., and D'Lima, D.D. (2011). Macroscopic and histopathologic analysis of human knee menisci in aging and osteoarthritis. Osteoarthritis and cartilage / OARS, Osteoarthritis Research Society 19, 1132-1141.

Petersen, W., Pufe, T., Starke, C., Fuchs, T., Kopf, S., Raschke, M., Becker, R., and Tillmann, B. (2005). Locally applied angiogenic factors--a new therapeutic tool for meniscal 
repair. Annals of anatomy $=$ Anatomischer Anzeiger : official organ of the Anatomische Gesellschaft 187, 509-519.

Pfaffl, M.W. (2001). A new mathematical model for relative quantification in real-time RT-PCR. Nucleic acids research 29, e45.

Pritzker, K.P., Gay, S., Jimenez, S.A., Ostergaard, K., Pelletier, J.P., Revell, P.A., Salter, D., and van den Berg, W.B. (2006). Osteoarthritis cartilage histopathology: grading and staging. Osteoarthritis and cartilage / OARS, Osteoarthritis Research Society 14, 13-29.

Rai, M.F., Patra, D., Sandell, L.J., and Brophy, R.H. (2013). Transcriptome analysis of injured human meniscus reveals a distinct phenotype of meniscus degeneration with aging. Arthritis and rheumatism 65, 2090-2101.

Reginster, J.Y. (2002). The prevalence and burden of arthritis. Rheumatology 41 Supp 1, 3-6. Segawa, Y., Muneta, T., Makino, H., Nimura, A., Mochizuki, T., Ju, Y.J., Ezura, Y., Umezawa, A., and Sekiya, I. (2009). Mesenchymal stem cells derived from synovium, meniscus, anterior cruciate ligament, and articular chondrocytes share similar gene expression profiles. Journal of orthopaedic research : official publication of the Orthopaedic Research Society 27, 435-441.

Seol, D., McCabe, D.J., Choe, H., Zheng, H., Yu, Y., Jang, K., Walter, M.W., Lehman, A.D., Ding, L., Buckwalter, J.A., et al. (2012). Chondrogenic progenitor cells respond to cartilage injury. Arthritis and rheumatism 64, 3626-3637.

Shen, J., Li, J., Wang, B., Jin, H., Wang, M., Zhang, Y., Yang, Y., Im, H.J., O'Keefe, R., and Chen, D. (2013). Deletion of the Transforming Growth Factor beta Receptor Type II Gene in Articular Chondrocytes Leads to a Progressive Osteoarthritis-like Phenotype in Mice. Arthritis and rheumatism 65, 3107-3119. 
Steadman, J.R., and Rodkey, W.G. (2005). Tissue-engineered collagen meniscus implants: 5to 6-year feasibility study results. Arthroscopy : the journal of arthroscopic \& related surgery : official publication of the Arthroscopy Association of North America and the International Arthroscopy Association 21, 515-525.

Steinert, A.F., Palmer, G.D., Capito, R., Hofstaetter, J.G., Pilapil, C., Ghivizzani, S.C., Spector, M., and Evans, C.H. (2007). Genetically enhanced engineering of meniscus tissue using ex vivo delivery of transforming growth factor-beta 1 complementary deoxyribonucleic acid. Tissue engineering 13, 2227-2237.

van Beuningen, H.M., Glansbeek, H.L., van der Kraan, P.M., and van den Berg, W.B. (1998). Differential effects of local application of BMP-2 or TGF-beta 1 on both articular cartilage composition and osteophyte formation. Osteoarthritis and cartilage / OARS, Osteoarthritis Research Society 6, 306-317.

van der Kraan, P.M., Blaney Davidson, E.N., and van den Berg, W.B. (2010). Bone morphogenetic proteins and articular cartilage: To serve and protect or a wolf in sheep clothing's? Osteoarthritis and cartilage / OARS, Osteoarthritis Research Society 18, $735-741$.

van der Kraan, P.M., Goumans, M.J., Blaney Davidson, E., and ten Dijke, P. (2012). Agedependent alteration of TGF-beta signalling in osteoarthritis. Cell and tissue research $347,257-265$

Webber, R.J., York, J.L., Vanderschilden, J.L., and Hough, A.J., Jr. (1989). An organ culture model for assaying wound repair of the fibrocartilaginous knee joint meniscus. The American journal of sports medicine 17, 393-400.

Woolf, A.D., and Pfleger, B. (2003). Burden of major musculoskeletal conditions. Bulletin of the World Health Organization 81, 646-656. 
Zhang, D., Cheriyan, T., Martin, S.D., Gomoll, A.H., Schmid, T.M., and Spector, M. (2011). Lubricin distribution in the torn human anterior cruciate ligament and meniscus. Journal of orthopaedic research : official publication of the Orthopaedic Research Society 29, 1916-1922. 
Figure legends:

Figure 1: Meniscus tissue histology and molecular composition. (A) Immunohistochemistry of collagen type I in healthier meniscus (arrow); insert: low magnification of a human healthier meniscus stained for collagen type I, the black line indicates the border between the vascular part on the right side and the avascular part on the left side. (B) Healthier human meniscus with an intact superficial zone and flattened cells (arrow). (C) Diseased meniscus with a completely degenerated superficial zone where only the round cells of the inner zone remain (arrow). (D) Calcifications and (E) cell clusters are signs of OA. (F) Immunohistochemistry of collagen type II in diseased meniscus (arrows); insert: low magnification of a human diseased meniscus stained for collagen type II. Magnification in A-F, bar $=150 \mu \mathrm{m}$; inserts bar $=1,7$ cm. (G) Heat map of the top 100 differentially expressed genes with p-values $<0.001$. The red color indicates up-regulated genes, and the blue color indicates down-regulated genes. The up-regulated genes in diseased meniscus compared with healthier tissue are: ACTR2, KRT, HSPD1, CALU, HSPA9, BAX, CCDC80, C17orf59, HSP90AB1, CDC42, HNRNPH1, FKBP9, MT1DP, G3BP1, CTTN, COL6A1, TAF15, ELAVL1, MCL1, AL, HSPA4, CXorf4OA CXorf4OB, TCP1, RP5, VHL, PRPF4, BAG5, RIOK3, FCF1, SPTLC1, APH1A, RNF170, RCN3, DENR, CAPZB, MBNL1, CASC4, ASB1, MRPS10, RAB23, PAAF1, FARSB, NASP, NUDC, ZNF346, RIOK1 and GAR1. The down-regulated genes in diseased meniscus compared with healthier tissue are: RNF103, SLC41A3, SNX19, GTFIP1, ERF, NBPF10 NBPF3, OSBPL10, ADAM15, KIAA0930, ZMAT3, AL845464.3, ANTXR1, ENDOD1, ZNFX1, GLT8D2, RP5-1022P6.4, ENG, POMZP3, C11orf95, LPCAT1, EPN2, POMZP3, RAB11FIP3, TBC1D2B, PCDHGC3, MMP14, TRPM4, HDAC5, ABL1, SOX9, Antxr1, BCAN, ZP3, ABR, SNX33, RPS6KA4, CXCR7, NCOR2, PLEC, GPC1, SEMA3C, PDGFRB, LUM, ACAN, CSPG4, OAS1, TIMP2, VCAM1, LAM5, PRSS54, PCDHB1 and FMOD. (H) Selected 
microarray data of high-interest genes (SOX9, PDFGFRB, ACAN, TIMP2 and FMOD) that were significantly down-regulated in cells derived from diseased tissue compared with cells from healthier meniscus tissue. Only COL6 is up-regulated. (I) Peptide abundance of selected genes shown as the mean of four experiments that analyzed cells that migrated from meniscus samples from healthier and diseased. Standard deviation (S.D.).

Figure 2: TGFB3, smads, runx2 and sox9 in human osteoarthritic meniscus tissue (A-F). (A) A healthier meniscus stained for TGFB3 compared with the sparse staining in diseased meniscus (B). (C) Sparse staining of smad1 in diseased meniscus tissue. (D) The pattern observed for TFGB3 (A,B) was also found for smad2/3 in healthier compared with diseased meniscus tissue. (F) Smad4 staining in healthier meniscus tissue. Magnification in A-F, bar = $150 \mu \mathrm{m}$. (G-K) Western blotting and quantification using ImageJ64 (G-K): (G) a-Tubulin shows equal loading; the first lanes of the blots corresponding to the dark grey bars in the graphs always show the healthier specimens. The diseased specimens are always found in the second lanes of the blots which correspond to the light grey bars in the graphs. A significantly greater level of smad2/3 in healthier meniscus tissue $(H)$. Runx2 is almost undetectable in healthier tissue, whereas it is significantly elevated in diseased meniscus (I). Sox9 showed no significant differences between tissue types (J). Smad4 was increased significantly in diseased meniscus (K). (L-O) Quantitative real time RT-PCR results: (L) TGFB3 mRNA is significantly increased in healthier meniscus tissue; however, runx2 mRNA levels are significantly reduced in healthier meniscus (M). (N) Sox9 mRNA is significantly reduced in diseased meniscus, which is in line with the significant reduction in collagen type II mRNA (O). *Significant differences $(p \leq 0.05)$; error bars denote the means \pm S.D. of three individual experiments for mRNA and Western blotting experiments. 
Figure 3: Cells from diseased human meniscus in vitro are similar to cells found in vivo: (A) Explant cell culture of cells migrating out of a diseased meniscus specimen. (B) Cells in P1 from the inner zone after collagenase digestion maintained a round phenotype in 2D culture. However, cells in P1 from the superficial zone from healthier menisci maintained their spindle shape (C). (D) Immunocytochemistry of the inner zone cells for collagen type I. (E) Collagen type I in the superficial zone cells. (F-I) Quantitative real time RT-PCR results from the cells in vitro. (F) TGFB3 mRNA levels are significantly higher in cells from healthier samples, which is consistent with smad2 $(\mathrm{G})$ and smad3 $(\mathrm{H})$ mRNA. No significant difference was observed for sox9 mRNA; however, a tendency toward higher levels in cells derived from healthier tissue was noted (I). (J-L) Immunocytochemistry of inner diseased meniscal cells positive for smad1 (J), note the punctuate paranuclear pattern, smad2/3 (K) and smad4 (L). (M-O) The human PCR array for the TGFB/BMP pathway revealed high levels of mRNA of inhibitors of the TGFB/BMP axis, for example, smad7 $(\mathrm{M})$ noggin $(\mathrm{P})$, follistatin $(\mathrm{O})$, in cells obtained from inner diseased meniscus. ${ }^{\star}$ Significant differences $(p \leq 0.05)$; error bars denote the means \pm S.D. of three individual experiments. Magnification in A-E and J-L, bar $=150 \mu \mathrm{m}$.

Figure 4: The cells from the inner zone of the diseased meniscus show progenitor cell characteristics and are multipotent. (A) FACS data showed that migratory cells from diseased meniscus explant cultures obtained without collagenase digestion expressed significantly more stem cell markers, including Stro1, CD29, CD105, CD106 (bars on left side), than cells obtained via collagenase digestion from a healthier meniscus (bars on right side). CD90 was found to be expressed equally in the two cell populations. (B-E) Chondrogenic differentiation in 3D alginate (3D) and with additional chondrogenic differentiation medium (3D + chon.diff.). 
(B) a-Tubulin loading control, the first lanes always represent the 2D control experiments, the second lanes always represents the 3D differentiation and the third lanes always represents the 3D differentiation together with the chondrogenic differentiation medium. (C) Production of sox9 is significantly increased under $3 D+$ chon.diff. conditions compared with the control in 2D and 3D cultures. (D) Runx2 was undetectable following 3D + chon. diff. culture. (E) Collagen type II was present after 3D culture and 3D+ chond. diff. culturing.

(F-H) Western blotting data were confirmed by mRNA results that revealed significantly higher levels of sox9 mRNA (F) and collagen type $\|(G)$ following 3D + chon. diff. culture compared with control cells in 2D culture. $(H)$ Collagen type I mRNA decreased significantly following 3D + chon.diff. culture. After osteogenic differentiation, cells become positive for alkaline phosphatase (I) and (J) osteocalcin. Adipogenic differentiation is indicated by positive Oil red staining (K). *Significant differences ( $\mathrm{p} \leq 0.05$ ); error bars denote the means \pm S.D. of three individual experiments. Magnification in $\mathrm{I}-\mathrm{K}$, bar $=150 \mu \mathrm{m}$.

Figure 5: Migration assays. (A) The strategy for an ex vivo migration assay. (A, top flasks). MPCs were GFP-labeled, enriched via FACS to 99\% and cultured. (A, middle flasks) Upon reaching $70 \%$ confluence, one side was scraped and a sample of diseased meniscus tissue was placed on the cell-free side. After 3-4 days, MPCs re-populated and migrated into the tissue sample. (A, bottom flasks) The tissues were washed with 1x PBS solution and transferred into a new flask, and after 7-10 days, some labeled and unlabeled MPCs migrated out again. The experimental stages were visualized using a fluorescence microscope. (B) The labeled cells adjacent to the diseased meniscus tissue. (C) Labeled MPCs in the sample of the diseased tissue after washing and transferal to a new flask. (D) Boyden chamber assay results showing that significantly more MPCs were attracted by 
human recombinant PDGF compared with controls. *Significant differences $(p \leq 0.05)$; error bars denote the means \pm S.D. of three individual experiments.

Figure 6: The influence of TGFB3 and BMP2 on the chondrogenic potential of MPCs. (A) aTubulin indicates equal loading of the gel lanes, also in this figure, the first lanes in the blots and the dark grey bars in the graphs represent control experiments, while the second lanes and the light grey bars represent the TGFB3 stimulation experiments. Following stimulation of MPCs with $10 \mathrm{ng} / \mathrm{ml}$ TGFB3 for $24 \mathrm{~h}$, total smad2/3 (B) was significantly increased and active p-smad2 was significantly up-regulated (C). In turn, sox9 increased significantly (D) and runx2 was reduced significantly (E). In contrast, following stimulation of MPCs with $10 \mathrm{ng} / \mathrm{ml}$ BMP2 for $24 \mathrm{~h}$, total smad2 was reduced significantly $(\mathrm{F})$, as well as sox9 $(\mathrm{G})$, shown by real time RT-PCR. *Significant differences ( $p \leq 0.05)$; error bars denote the means \pm S.D. of three individual experiments.

Figure 7: Knock-down of runx2 (A-D) and overexpression of smad1 and smad2 (E-I). (A) aTubulin indicates the equal loading of the gel lanes and was used for the quantification with ImageJ64, the first lanes of the blots always represents the controls as do the grey bars in the graphs, while the second lanes of the blots and the light grey bars of the graphs always represents the runx2 knock-down. (B) Following siRNA knock-down (KD) of runx2, the protein was undetectable (lane 2) compared with the control (lane 1). In turn total smad2/3 increased significantly (C) and enabled the detection of p-smad2 (D). (E) a-Tubulin indicates equal lane loading and was applied for quantification, the first lanes of the blots always represents the controls as do the dark grey bars in the graphs. The second lanes and the light grey bars represent smad1 overexpression (OE). Finally the third lanes of the blots and 
the black bars of the graphs represent the OE of smad2. (F) Smad1 OE is seen in lane 2. (G) Smad2 OE is seen in lane 3. $(\mathrm{H}) \mathrm{OE}$ smad1 and smad2 results in a significant up-regulation of sox9. (I) OE of smad2 results in a greater reduction of sox9 as does OE of smad1. 
95
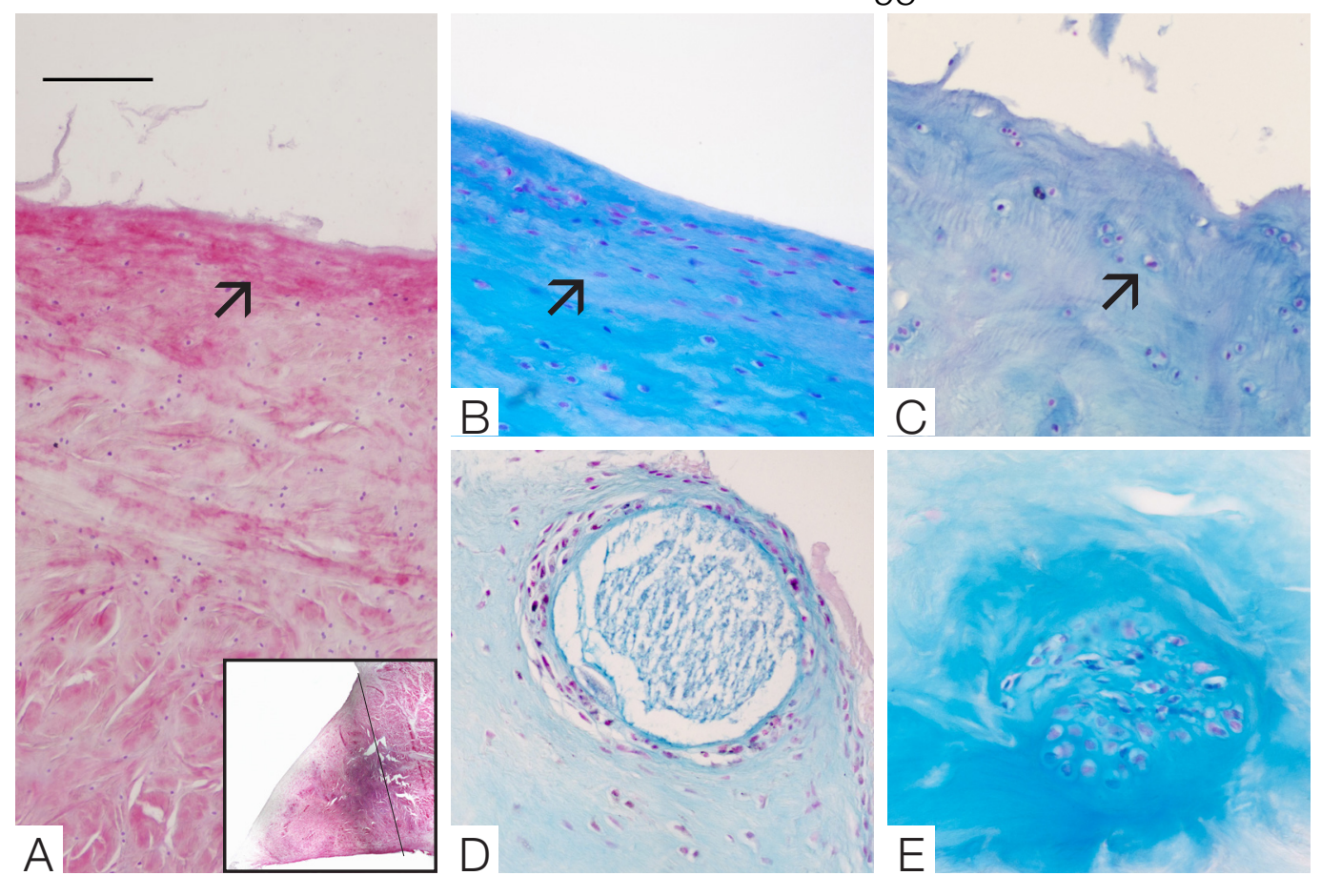

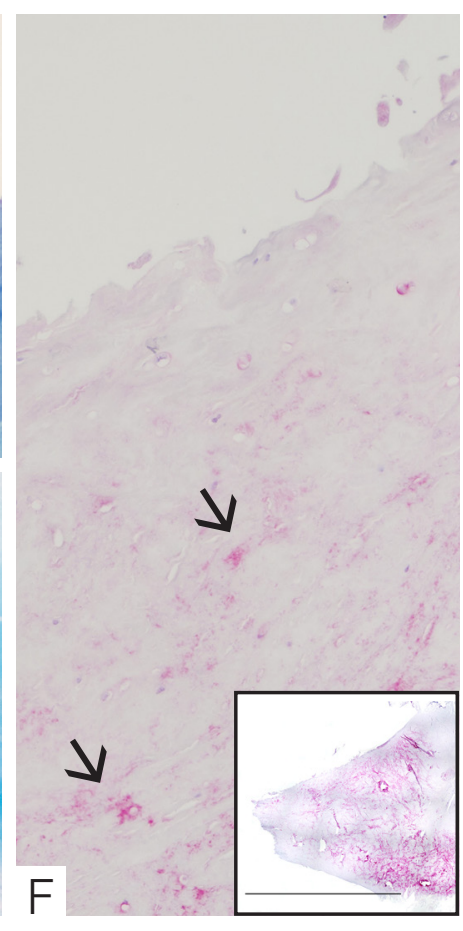

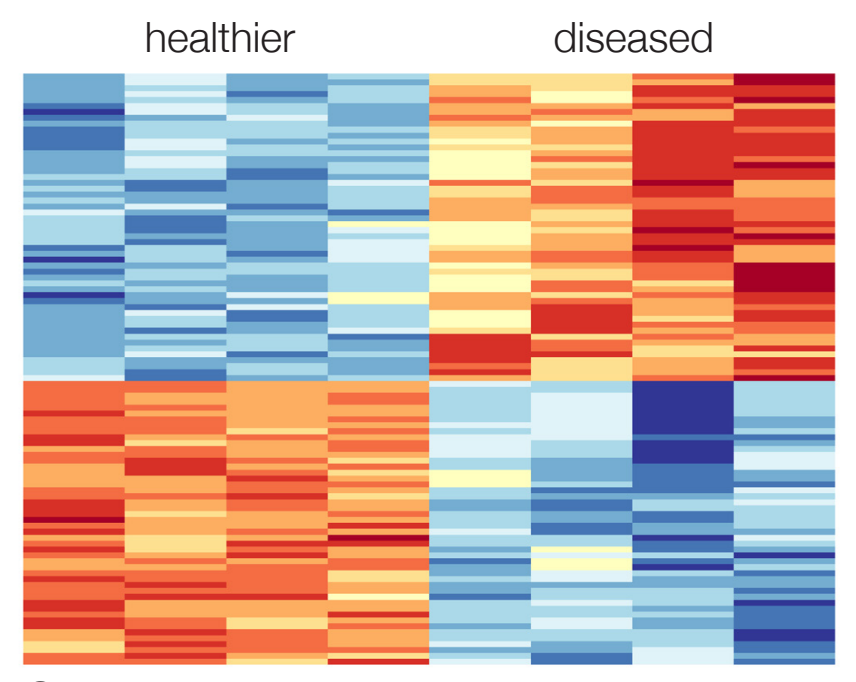

\begin{tabular}{lccr}
\hline Accession No & Gene ID & Fold change & \multicolumn{1}{l}{ P value } \\
\hline NM_000346 & SOX9 & $-1,090$ & 0,00010395 \\
NM_002609 & PDGFRB & $-1,538$ & 0,000520006 \\
NM_001135 & ACAN & $-1,634$ & 0,000297344 \\
NM_003255 & TIMP2 & $-1,877$ & $5.668051243 e^{-05}$ \\
NM_002023 & FMOD & $-2,396$ & 0,00533354 \\
NM_001848 & COL6A1 & 0,956 & 0,000535283 \\
\hline
\end{tabular}

$\mathrm{H}$

\begin{tabular}{lccccr}
\hline Name & IPI No & $\begin{array}{c}\text { Peptide } \\
\text { abundance } \\
\text { healthier }\end{array}$ & S.D. & $\begin{array}{c}\text { Peptide } \\
\text { abundance } \\
\text { diseased }\end{array}$ & S.D. \\
\hline SMAD2 & IPI00019548 & 20,7 & 7,9 & 64,2 & 21,3 \\
SMURF2 & IPI00329664 & 16,2 & 3,7 & 30,9 & 7,9 \\
COL1A1 & IPI00304962 & 398,4 & 22,0 & 409,2 & 83,5 \\
ACAN & IPI00027377 & 100,7 & 25,8 & 86,7 & 54,6 \\
COL6A2 & IPI00983601 & 34,4 & 10,4 & 28,5 & 6,4 \\
PDGFRB & IPI00015902 & 30,2 & 11,9 & 37,3 & 6,8 \\
BGN & IPI00010790 & 55,1 & 19,1 & 40,7 & 21,9 \\
COMP & IPI00028030 & 32,31 & 15,5 & 31,0 & 16,3 \\
\hline
\end{tabular}


healthier, TGFB3

diseased, TGFB3

diseased, smad1
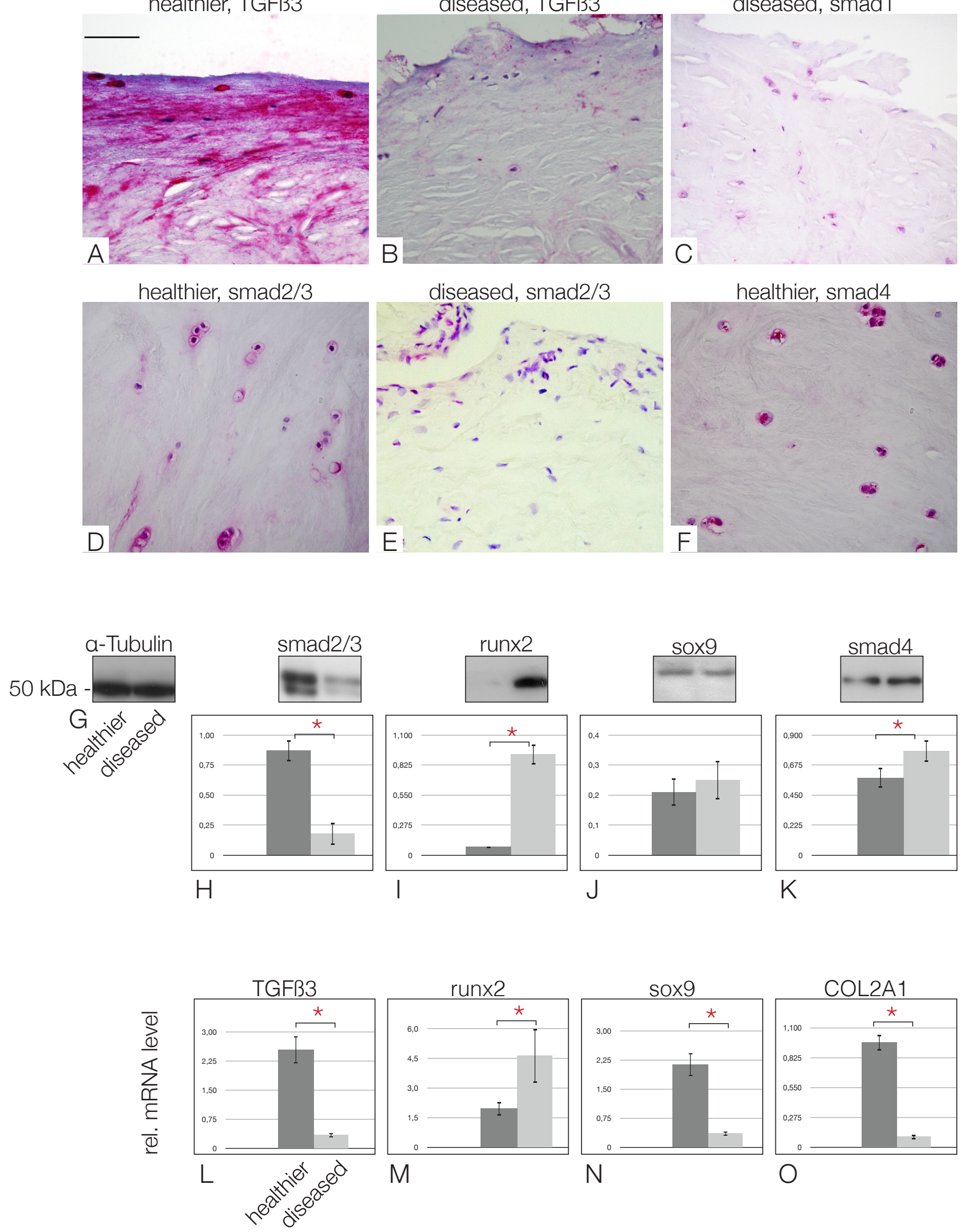

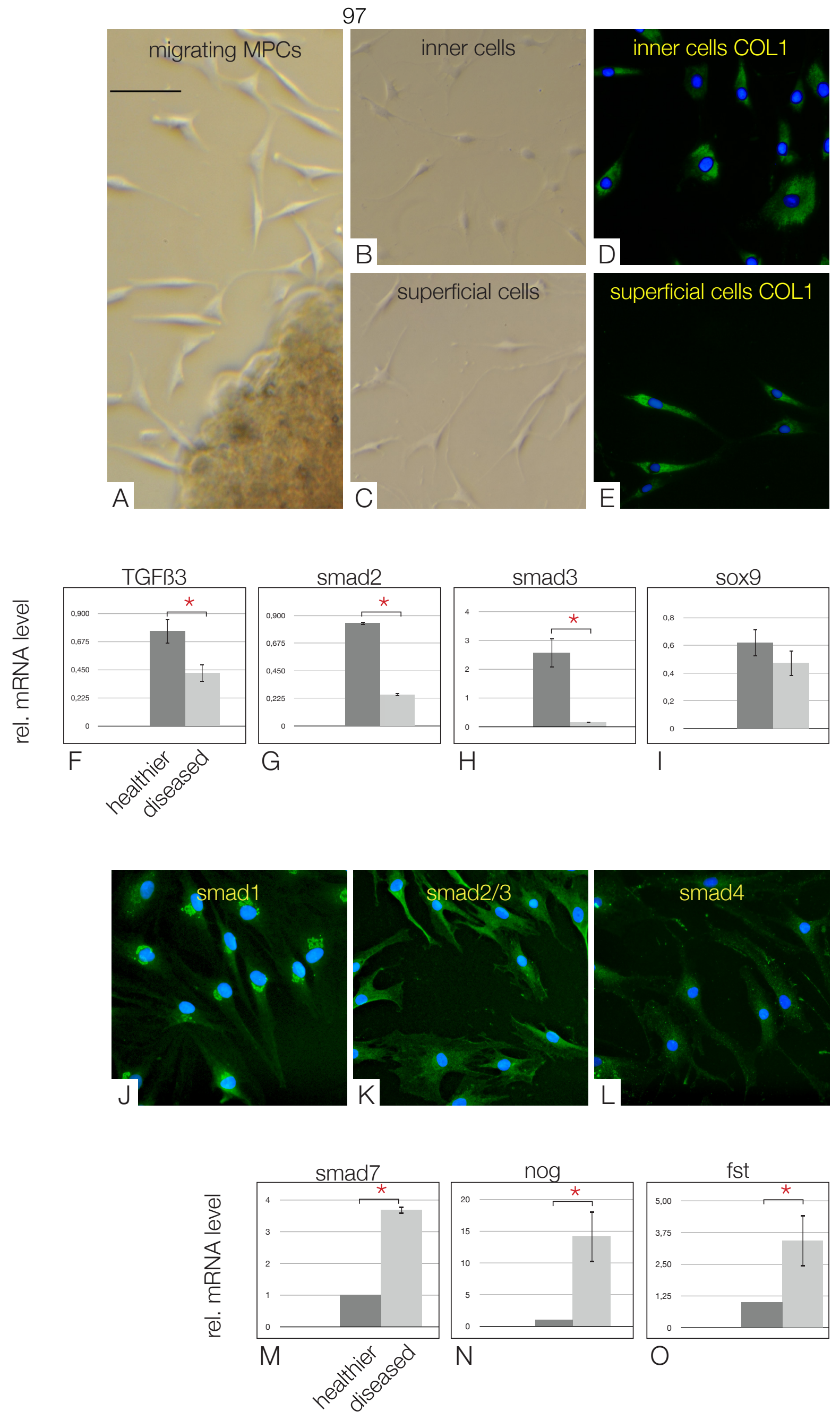

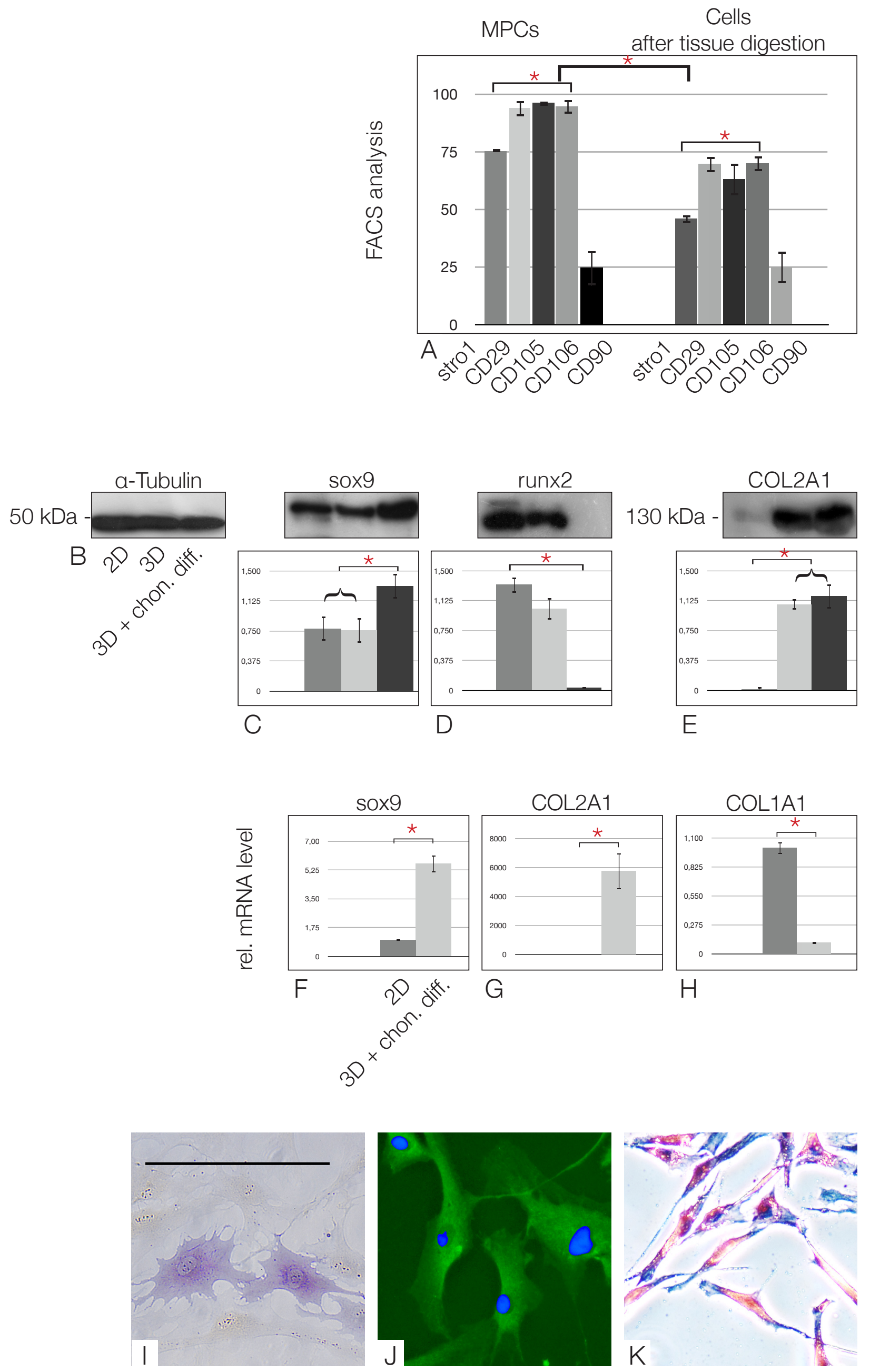
MPCs

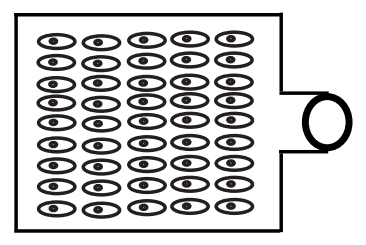

meniscus

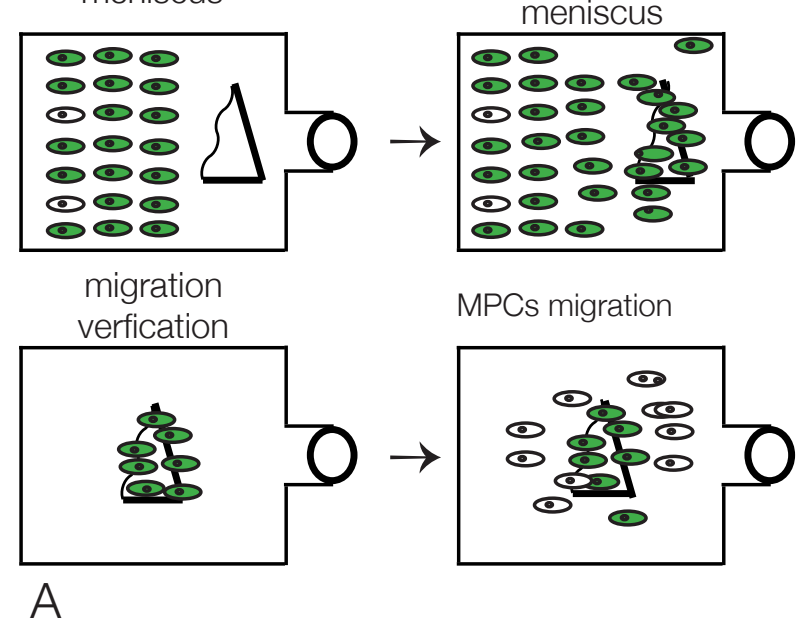

GFP labeled

100

1

migration into

meniscus

ब $\infty 6$

000

s migration
O00
99
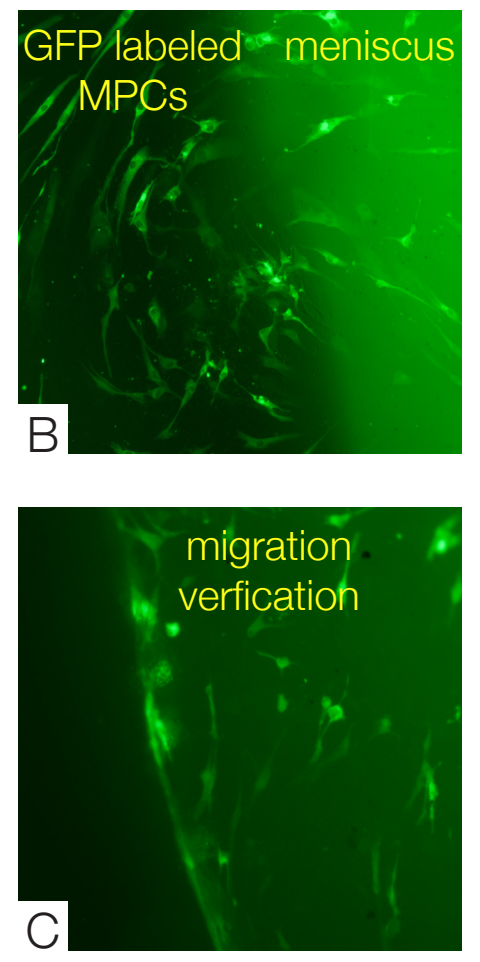

$\mathrm{C}$

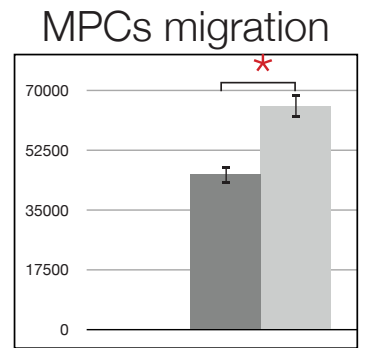

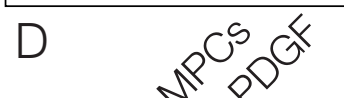
$c^{2}$

\section{Figure 5}


Figure 6
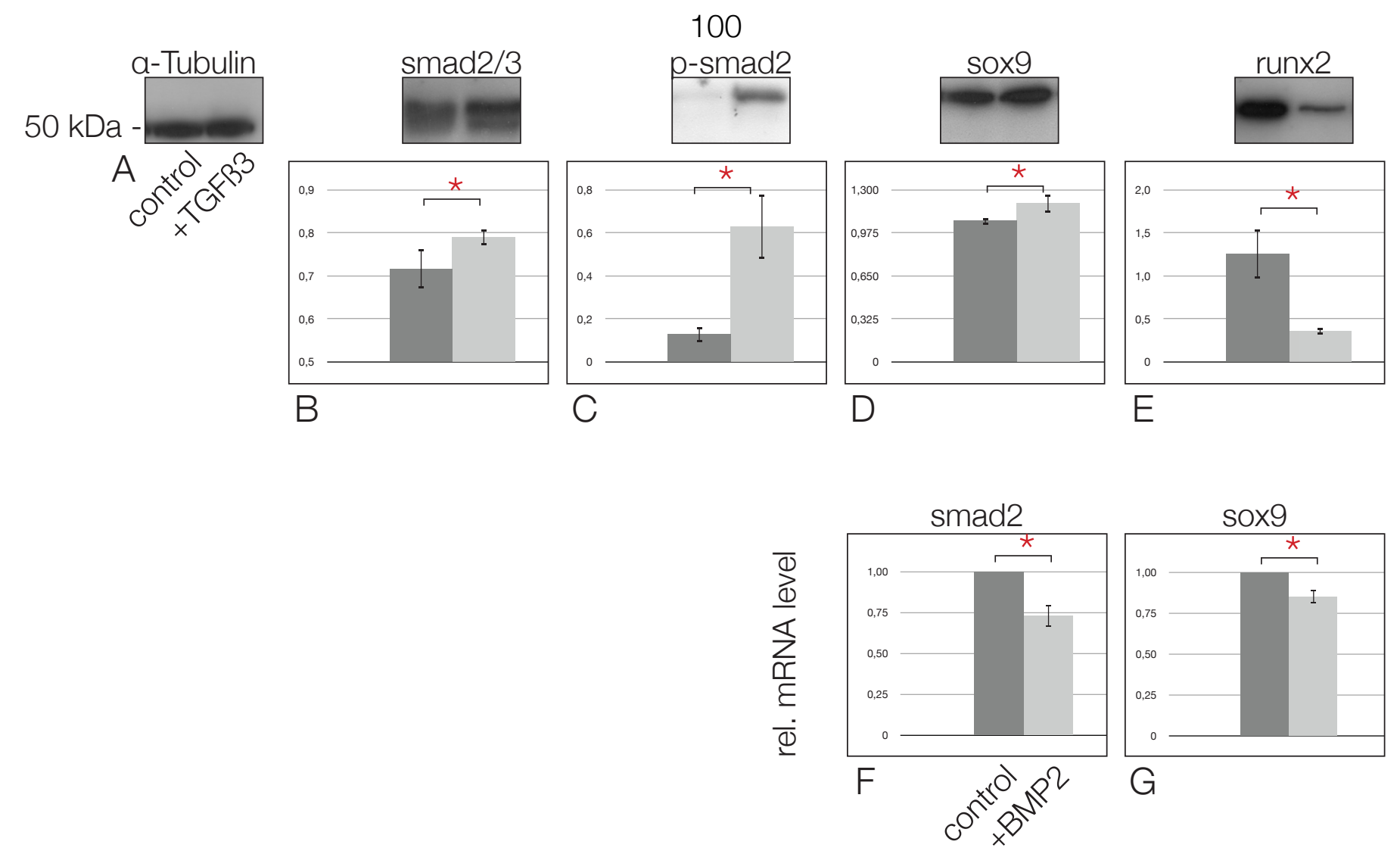
Figure 7
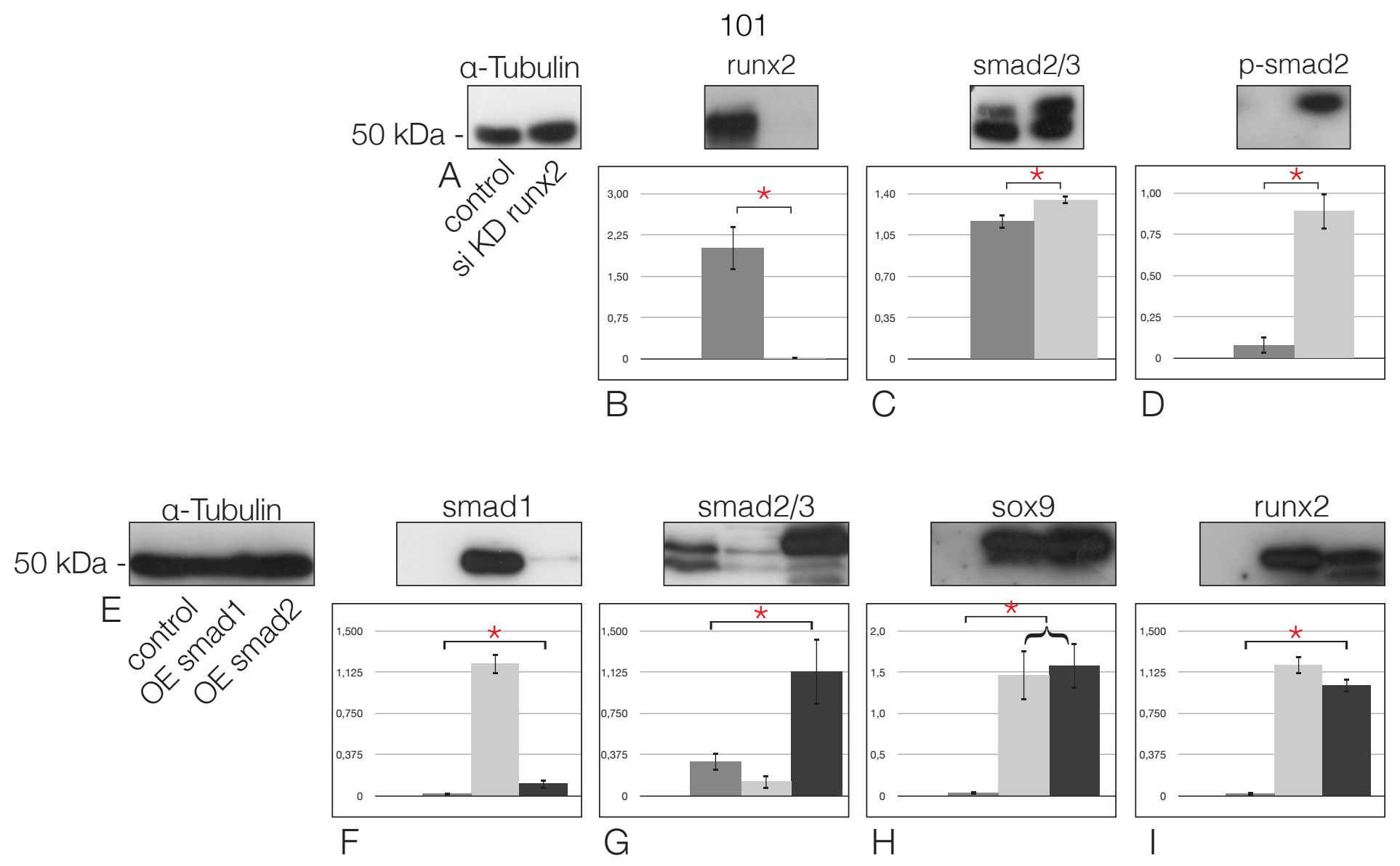


\section{Chapter 5}

Identification and characterization of mouse meniscus progenitor cells in vitro and ex vivo

Hayat Muhammad ${ }^{1}$, Matz Menrath ${ }^{1}$, Boris Schminke ${ }^{1}$, Vicki Rosen ${ }^{2}$ and Nicolai Miosge ${ }^{1 \#}$

${ }^{1}$ Tissue Regeneration Work Group, Department of Prosthodontics

Medical Faculty, Georg August University

D-37075 Goettingen, Germany

2Developmental Biology, Harvard School of Dental Medicine,

188 Longwood Avenue, Boston, MA 02115, USA

"Corresponding author

Hayat Muhammad's contribution: Study conception, immunohistochemistry, histology, cell culture, FACS analysis and manuscript preparation. 
Identification and characterization of mouse meniscus progenitor cells in vitro and ex vivo Hayat Muhammad ${ }^{1}$, Matz Menrath ${ }^{1}$, Boris Schminke ${ }^{1}$, Vicki Rosen ${ }^{2}$ and Nicolai Miosge ${ }^{1 \#}$ ${ }^{1}$ Tissue Regeneration Work Group, Department of Prosthodontics, Medical Faculty, Georg-August-University, D-37075 Goettingen, Germany

2Developmental Biology, Harvard School of Dental Medicine, 188 Longwood Avenue, Boston, MA 02115, USA

\#Corresponding author

Tissue Regeneration Work Group, Department of Prosthodontics Medical Faculty, Georg-August-University; Robert Koch Straße 40, 37075 Goettingen, Germany, Phone: 00495513933927, Fax: 0049551398621, Email: nmiosge@gwdg.de

Keywords: mouse meniscus progenitor cells, osteoarthritis, stem cells

\section{Abstract}

The meniscus is a fibrocartilagenous tissue in the knee joint. It gives stability to the joint and is considered as a safeguard to protect the femorotibial articular cartilage. Meniscus degradation ultimately leads to osteoarthritis. The major part of the meniscus is avascular, which has difficulties to self-repair once damaged. Meniscus removal always results in osteoarthritis. Therefore, new approaches are required to repair or regenerate the damaged meniscus to result in the same biomechanical features as healthy normal tissue. Here, we are introducing mouse meniscus progenitor cells, which are positive for the stem cells markers, e.g., CD29, CD73, CD90, and CD44. These cells are multipotent and could be differentiated at least to two mesenchymal lineages, adipocytes and cells of the osteoblastic lineage. Additionally, we also confirmed the localization of these cells in vivo. Furthermore, histological results clearly depict the structural differences between the human and the mouse menisci in terms of their cellular and matrix composition. The superficial zone with its flattened cells in humans, stained more for collagen type I, however this staining is absent in the mouse meniscus. These initial findings will help to explore and understand the nature of progenitor cells and their involvement in regeneration of meniscus tissue. 
Introduction

In the United States about 27 million people are affected by osteoarthritis (OA). OA is a major cause of disability in adults (Centers for Disease \& Prevention, 2010; Lawrence et al, 2008). There are numerous risk factors leading to OA, for example, age, obesity, genetics, trauma, joint anatomy, and the occupational history of an individual (Blagojevic et al, 2010). While OA is multifactorial, one of the key causes of OA is the damage of the meniscus. The meniscus protects the cartilage and works as a shock absorber in the knee joint. Meniscus tissue exhibits intrinsic repair capabilities; however these are restricted to the red, the vascular part. While damage of the white, avascular part does not have this intrinsic capability. Partial meniscectomy cannot stop the degradation process (Arnoczky \& Warren, 1983; Hasan et al, 2013; Walker \& Erkman, 1975).

To repair the damaged or diseased meniscus tissue in all aspects of a normal native meniscus is a great challenge. It is also difficult to stop the secondary symptoms of the injured or damaged meniscus tissue such as calcification (Noble \& Hamblen, 1975). The present surgical procedures are satisfactory at a temporary level but can't prevent the deleterious effects resulting in OA (McDermott \& Amis, 2006). There are other alternatives available such as the application of mesenchymal stem cells (MSCs). However, in practice, MSCs face two major problems: first the inappropriate production of the extra cellular matrix (ECM) and the second obstacle is the occurrence of hypertrophy (Mauck et al, 2006; Pelttari et al, 2006). There are attempts to overcome these problems, for example, via co-culturing of MSCs with chondrocytes in hydrogel (Bian et al, 2011). Another idea was to culture the MSCs together with meniscus derived cells to encourage the differentiation of MSCs towards a meniscal phenotype without hypertrophy (Cui et al, 2012). It is reported that the bone marrow derived MSCs enhanced the healing process in combination with a fibrin glue (Ferris et al, 2012). Interestingly, not only the bone marrow derived MSCs are tested as potential candidate for meniscus tissue regeneration, but also other tissue sources, such as, synovial derived MSCs are considered. The synovial membrane-derived MSCs are being described as growing fast and exerting a high chondrogenic potential (Katagiri et al, 2013; Sakaguchi et al, 2005).

More recently, Shen et al. published that meniscus-derived stem cells have a tremendous ability for articular cartilage repair, however, this was tested in a rabbit 
model, which has a very high self-renewal capacity (Shen et al, 2013). The concept of progenitor cells in situ from diseased tissues is gaining extensive interest in regenerative medicine and we previously introduced chondrogenic progenitor cells (Koelling et al, 2009) from cartilage of late stages of OA. We have also investigated that human menisci harbor progenitor cells that are governed by the canonical TGF $\beta$ pathway (manuscript submitted). Meniscus degeneration exposes the knee cartilage directly to high body pressure and ultimately damages the tissue and results in OA. To date, all the experimental efforts for the meniscus tissue engineering or regeneration are encouraging but seem not to be promising in the long term. Allografts or the bio-engineered meniscal substitutes seem to be only filling the space void after meniscal removal, however, the clinical and $\mathrm{MRI}$ evaluation does not show a satisfactory protection against the development of OA (Hommen et al, 2007). For the first time, we are introducing tissue specific, mouse meniscus progenitor cells (MMPCs). We characterized these cells in vivo with the help of immunohistochemical techniques and in vitro using various biochemical techniques. The MMPCs are found positive for well-known stem cell markers such as CD44, CD29, and CD73. They are multipotent and could be differentiated into adipocytes and cells of the osteoblastic lineages. We believe that our initial findings will help to develop new strategies of cell biological therapies for meniscus repair in situ.

Materials and methods

Sample preparation

The treatments of the animals were performed according to the principles and laws of the Animal Welfare Act of the County of Lower Saxony, Germany. The samples were gently handled using sterile instruments and all the surgical steps were carried out under sterile conditions. First, muscles and ligaments were removed under a stereo microscope (Leica, EZ4D). After the removal of the cruciate ligaments of the distal femur, the tibial plateau was detached easily with the help of tweezers. Some meniscus pieces were immediately transferred to the cell culture medium for explant cell culture. Others menisci and surgically untouched complete knee joints were decalcified for immunohistochemical experiments. 
Cells isolation and cells culture

The grown out cells of the explant culture were cultured in a standard cell culture medium Dulbecco's modified Eagle's medium (DMEM) with the 10\% fetal bovine serum (GICO, lot number 41F2061K). Additionally, supplemented with penicillin/streptomycin (50,000 U/50mg) and $10 \mathrm{mM} \mathrm{L-glutamine.}$

Multipotent differentiation

The nearly confluent flask (75\%) was subjected to the osteogenic differentiation under the influence of the proper induction medium, NH OsteoDiffMedium (Miltenyi Biotec), with the combination of dexamethasone, ascorbic acid, and B-glycerophosphate. The cells were encouraged toward the adipogenic lineage using $\mathrm{NH}$ AdipoDiffMedium, which is a mixture of active compounds, such as, hydrocortisone, isobutylmethylxanthine, and indomethacin.

Cytochemistry

To evalutate the osteogenic differentiation, alkaline phosphatase staining was performed (86-C, Sigma), and oil-red staining was applied for fat detection in adipocytes after adipogenic differentiation (Koelling et al., 2009).

Antibodies for immunohistochemistry, immunocytochemistry and Western blots

For the immunohistochemistry we followed the PAP method. The monoclonal antibodies against collagen type I (M-38) and collagen type II (CIICl), both were obtained from the Developmental Studies Hybridoma Bank, University of lowa, USA. The monoclonal antibodies against CD29 were purchased from abcam, Cambridge, UK and alpha-tubulin from Sigma-Aldrich, St. Gallen, Switzerland. Polyclonal antibodies against CD44 were ordered from abcam, Cambridge, UK and anti-CD73 were purchased online, antibodiesonline, Atlanta, USA.

We also performed the immunoreactions in the absence of primary antibodies, as negative control and all images are representatives of three separate individual experiments. 
Immunofluorescence microscopy

The primary migratory cells were transferred in the P1 stage to 96 well plates and fixed by $70 \%$ ethanol after $16 \mathrm{hrs}$ culture. Further incubated with $100 \mu \mathrm{l}$ of primary antibody (1:50 in PBS) for $1 \mathrm{hr}$ at RT in the dark. When necessary, incubation with secondary fluorescence-coupled antibody (1:500 in PBS) for 20 min at RT followed. Finally, stained with DAPI, the cells were examined using a fluoresence microscope. Images were captured using a Nikon D-90 camera (Nikon, Düsseldorf, Germany).

RNA extraction from cultured cells, cDNA synthesis and quantitative real-time PCR

Primary cells of P1 were dissolved in RLT buffer and RNA was isolated according to the manufacturer's instructions (RNeasy Mini Kit; Qiagen, Chatworth, CA, USA). RNA was converted into cDNA using Qiagen QuantiTect Reverse Transcription Kit, following the manufacturer's instructions. The total volume of the PCR reaction was set to $10 \mu$, containing $5 \mu$ l of RealMasterMix (2.5x) (Eppendorf, Hamburg, Germany), $2 \mu$ primers (20 pmol), $1 \mu \mathrm{l}$ (1ng) of cDNA and $2 \mu \mathrm{l}$ of RNase free water. Primers were designed with the help of online primer3 software.

\begin{tabular}{|l|l|l|l|}
\hline Gene ID & Forward primer & Reverse primer & Accession \\
\hline CD29 & cgtgttgggaggcactgtgaatgta & gcattcacaaacacgacacctgca & NM_010578.2 \\
\hline CD73 & ccttcctctcaaatccagggacaa & acttctttggaaggtggatttcctg & NM_011851.4 \\
\hline
\end{tabular}


FACS analysis

The meniscus derived cells were suspended in PBS with fluorescence-coupled antibodies as described earlier, using a dilution of $1 \mu$ antibodies/ in $100 \mu$ l buffer with one million cells at RT for $1 \mathrm{hr}$ in the dark, washed twice by PBS, centrifuged for 10 minutes at $800 \mathrm{rpm}$ and finally were subjected to FACScane (Becton Dickinson, Mountain View, CA), as described in detail elsewhere (Koelling et al, 2009). A total of, approximately, 10,000 living cells were detected, and data were interpreted using WinMDlv2.9 software. We also performed further analyses with help of program Cell Quest Pro 2000.

Immunoblotting

Total proteins were extracted in $5 \mathrm{M}$ guanidine hydrochloride with protease inhibitors. Proteins were precipitated in ethanol and washed with PBS. Precipitation steps were repeated and finally proteins were dissolved in PBS (0.4\% SDS). For protein separation SDS-PAGE was performed. All the gels were mixed keeping $6 \%$ acrylamide in the stacking gel and $10 \%$ in the running or the separating gel. Further, the blotted membranes were blocked with $5 \%$ milk to avoid unspecificity. The immunoreactions were performed on the blotted membranes using primary antibodies over night at $4^{\circ} \mathrm{C}$. The next day membranes were washed with 1X TBST solution and probed with secondary antibodies for $1 \mathrm{hr}$ at RT. The protein bands were visualized via ECL reaction. 
Results

Histological features of mouse meniscus

Anatomically, the menisci are fixed in the knee joint by ligaments and muscles. The meniscus is sandwiched between the femur and tibia. To isolate the specimen, first of all, ligaments were removed and then the complete femur was detached easily. Finally, the C-shaped meniscus became prominent on the tibia under the stereomicroscope (Fig. 1A). After extraction from the tibia, mouse menisci revealed calcified features at both terminal ends (Fig. 1B). Longitudinal sections stained unevenly for collagen type II (Fig. 1C) and stained highly for collagen type I (Fig. 1D). Complete mouse knee joints were embedded in paraffin block and sections were stained with hematoxylin-eosin (Fig.1E). No signs related to OA, for example, no surface fissures in the articular cartilage (Fig. 1F) nor breaks in the osteochondral junction (Fig. 1I) were observed. However, the cross sectioning of mouse meniscus brings a pore to light (Fig. 11), perhaps due to the calcified region, as was observed in the extracted mouse menisci (Fig. 1B). The immunohistochemistry results revealed that collagen type II is restricted to the inner and the pseudo-superficial zone of the meniscus (Fig. 1G). In contrast collagen type I was detected mainly in the outer part of the meniscus (Fig. 1H).

Mouse meniscus explant-cultured derived cells exhibit stem cell-like characteristics

Migratory cells derived from explant-cultures acquired a flattened phenotype (Fig. 2A). However, they lost their flattened phenotype upon encapsulation in the three-dimensional network of alginate (Fig. 2B) and turned into round cells (Fig. 2C). The well-known cell surface antigens, so-called stem cell markers, for example, CD44, CD90, and CD73 were investigated in vitro via immunocytochemistry, and in vivo using immunohistochemical techniques (Fig. 2D). The cells derived from meniscus via explant culture were subjected to FACS analyses for the stem cell markers. Furthermore, these cells were differentiated to adipocytes and cells of the osteoblastic lineage. The differentiated cells were evaluated by lineage specific staining, for example, the adipocytes were identified by oil-red stain (Fig. 2E+F). The osteoblasts were found to be positive for alkaline phosphatase staining (Fig. $2 \mathrm{G}+\mathrm{H}$ ). The control experiments, without induction media, remained negative for oil-red staining (Fig. $2 \mathrm{I}+\mathrm{J}$ ) and no positive staining was observed 
for alkaline phosphatase (Fig. $2 \mathrm{~K}+\mathrm{L}$ ) in control reactions. The FACS data analyses revealed a high incidence of stem cell marker expression, which are related to the mesenchymal origin, for example, CD73, CD44 and CD29. However, these cells showed immuno-negativity for hematopoietic progenitor stem cells marker such as CD117 and CD34 (Fig. 2M).

Investigation of stem cell markers in vitro and in vivo

Paraffin embedded mouse meniscus sections were used for immunohistochemistry and staining was observed for stem cells markers, such as, CD44 (Fig. 3A), CD73 (Fig. 3B) and CD29 (Fig. 3C). Further, in vivo, CD44 (Fig. 3A) and CD73 (Fig. 3B) positive cells were seen, more in the pseudo-superficial and less in the inner zone. However, CD29 positive cells (Fig. $3 \mathrm{C}$ ) were found slightly more in the inner zone of the mouse meniscus. The control slides (Fig. 3D) without primary antibodies did not show immuno-positive cells. The expression of CD73 mRNA was found to be up-regulated compared to CD29 mRNA (Fig. 3E). As described earlier (Fig. 2D), these stem cells related antigens were localized in vitro using immuno-cytochemical techniques (Fig. 3F). For Western blot analyses, blotted membranes were stained with Coomassie brilliant blue (Fig. 3G). Further, Membranes were probed with anti-CD29 antibody; a desired protein band was identified at $135 \mathrm{kDa}$ (Fig. 3H). After stripping, the membrane was re-probed with CD44 antibody and a protein band was visualized by ECL reaction at $45 \mathrm{kDa}$ (Fig. 3l).

\section{ECM differences of mouse and human menisci}

Interestingly, the mouse menisci were found to be different from human in terms of their cellular and ECM composition. For example, immunohistochemistry revealed that collagen type $I$ is the major component of human meniscus and distributed evenly throughout the meniscus, in the outer (red part) and inner (white part). However, more intensively stained the superficial zone (Fig. 4E). In contrast, in mouse meniscus collagen type $\mathrm{I}$ is restricted to the outer part and is not present in the inner part (Fig. 4F). In short, human meniscus can be divided into four major zones (Fig. 4A). Mainly, there are two superficial zones (S), one inner avascular, white part (W) and one outer vascular, red part (R). The mouse meniscus is not this complex and possesses only two zones (Fig 1 B), one outer vascular, white part (W) and the inner avascular, red part (R). The extra two 
superficial zones, as in human, with its unique layer of multiple flattened cells (Fig. 4C) are absent in the mouse. In contrast, the surface region of the mouse meniscus exhibits very few flattened cells and therefore it can be termed, pseudo-superficial zone (Fig. 4D). 


\section{Discussion}

Mouse meniscus tissue harbors progenitor cells

Others and we previously introduced the existence of progenitor cells in the late stage of OA cartilage (Koelling et al, 2009; Seol et al, 2012). We observed that after 7-9 days cells migrated out of the explant culture. The FACS analyses and immunocytochemistry confirmed the expression of cells surface antigens, so called stem cells markers, for example, CD44, CD73, CD29, and CD90. However, these migratory cells were found to be negative for hematopoietic progenitor stem cell markers, for example, CD117 and CD34. Our findings suggest that these cells are different from the cells isolated by other researchers (Osawa et al, 2013). For example, Osawa et al. isolated meniscus cells from the red vascular part via collagenase digestion. They found them positive for hematopoietic stem cells markers, as one would expect. This indicates that the possible source of these cells could be blood vessels or pericytes. Our cells are derived from the inner avascular part of the meniscus. Further, these cells showed multipotency; becoming positive for markers of the adipocytes and osteoblastic lineage after induction with the proper differentiating media. Here, we are introducing for the first time mouse meniscus progenitor cells. However, Furumatsu et al., have characterized the cells derived from the inner part of the human meniscus via collagenase digestion (Furumatsu et al, 2011). Surprisingly, we found the cells derived from the inner part of human meniscus via collagenase digestion exhibit less stem cell characteristics as compared to the cells derived via explant-culture from the same region of human meniscus. It suggests that migratory progenitor cells are different from residing cells. However, mouse meniscus progenitor cells share the spindle phenotype and clonal growth characteristic of CPCs (Koelling et al, 2009).

Taken together, the confirmation of stem cell markers, multipotency, clonicity and migratory potential allowed us to introduce the cells as 'mouse meniscus progenitor cells' (MMPCs).

Meniscus progenitor cells in ex vivo and in vitro

Adult stem cells or progenitor cells can be found in mature developed tissues. These progenitor cells in situ are involved in regeneration phenomena after trauma or disease. 
For instance, hematopoietic stem cells are found in bone marrow as a major pool to replenish the circulating blood cells. In line with this concept, the adult stem cells have been investigated in other adult mesenchymal tissues, for instance, blood, adipose, skin, mandible, trabecular bone, muscle, and synovial membrane (Muhammad et al, 2013; Punwar \& Khan, 2011; Till \& McCulloch, 2012). Here we investigated the existence of progenitor cells in healthy mouse meniscus tissue. The MMPC were found to be positive for stem cells markers (CD44, CD73, CD29, and CD90) and their confirmation were checked in all aspects, for example, at the transcription level by PCR, at the translation level by Western blot, immunohistochemistry and immunocytochemistry.

Structural overlap and differences of mouse and human menisci

Proffen et al. recently published a comparative study of menisci from different species. They discussed in detail the different anatomical and morphological aspects of seven species: human, sheep, goat, cow, dog and rabbit (Proffen et al, 2012). Here, we observed the structural features of mouse menisci in terms of their cellular and matrix composition. It has been shown histologically that the human menisci possess two major parts, in terms of their vascularity: the vascular (red) outer part, and the avascular (white) inner part (Pauli et al, 2011; Zhang et al, 2011). Furthermore, the inner part has two superficial zones and both are exposed to the surface of femero-tibial's articular cartilage. The superficial zone cells in human menisci are flattened in shape and are stacked in a multilayered fashion. Interestingly, a superficial zone as such is absent in mouse menisci. One can speculate that, a superficial zone only develops under a large total body weight, which exerts pressure on the superficial zone cells to acquire a flattened phenotype like in human.

Healthy mouse meniscus compared to less diseased human meniscus shows more or less the same histological features. However, there are slight differences, for instance, mouse meniscus is relatively more calcified then the human meniscus. In the human meniscus, the superficial zone and the inner part were uniformly stained for collagen type I. In contrast, the mouse meniscus collagen type I is only restricted to the outer red part of the meniscus. 
Surprisingly, degenerated human meniscus in the late stages of OA shares feature with healthy mouse meniscus. In the late stage of OA, the superficial zone of human meniscus is completely lost. The phenotypes of the cells of the human inner part, round chondrocyte-like cells are similar to the cells found in the pseudo-superficial zone of the inner part of mouse meniscus.

In summary, mouse meniscus harbors multipotent progenitor cells which can be localized in vitro and vivo. In addition, mouse meniscus is distinctively organized tissue, only exhibits a pseudo-superficial zone without flattened cells as in human meniscus. Our initial findings will help to understand and explore more about progenitor cell biology regarding meniscus tissue regeneration.

Figure Legends

Fig.1.

Structural aspects of mouse meniscus tissue (A-D): (A) Micro-surgical excisions of the mouse menisci were performed under the stereomicroscope. After the femoral condyle displacement, the C-shaped meniscus was seen on the tibia. (B) The extracted meniscus revealed a translucent appearance with a hard globular structure at both horns. (C) Longitudinal section of meniscus stained for collagen type I. (D) In contrast, the following section stained less for collagen type II. (E-F and I) H.E. staining of a healthy mouse knee joint $(E)$, magnified image of the complete joint revealed neither surface fissures nor breaks in the tidemark (F). (G) The cross section of a meniscus stained for collagen type II found to be restricted to inner part and, as expected, also seen in the hyaline cartilage tissue. $(H)$ In contrast, collagen type I staining only detected in the outer part of the meniscus, while the healthy hyaline cartilage tissue stained less, as expected. 
Fig. 2

Explant cell culture and 3D cell culture of MMPCs (A-C): (A) MMPCs migrating out of an explant culture. Cells show a flattened phenotype in two dimensional cell culture. (B) However, when cells were encapsulated in alginate, in a three-dimensional cell culture, they turned into a round phenotype (C). MMPCs, express stem cells marker and are multipotent (D-M): (D) These cells express stem cell markers CD44, CD90 and CD73 in vitro, and CD44 was in vivo (asterisk).

MMPCs are multipotent (G-H): Cells were cultured in the presence of adipogenic medium and were evaluated with oil-red staining. (E-F) In contrast, cells in the absence of stimulating medium have shown negativity for oil-red staining. (K-L) Cells induced with osteogenic medium revealed positive alkaline phosphatase staining. (I-J) In contrast, cells without the induction medium remained negative for alkaline phosphatase staining. (M) Furthermore, the FACS analyses also have confirmed the expression of stem cells markers, as shown for CD44 and CD73.

Fig. 3

Stem cells markers ex vivo (A-D): Immunohistochemical staining was performed for stem cells marker CD44 (A), CD73 (B) and (C) CD29. Interestingly, immunopositivity was seen in the cells, predominantly in the superficial zone of the meniscus tissue. (D) The control slides, without primary antibodies exposure, showed no staining. (E) The expression of stem cell markers (CD79 and CD73) were detected at the transcription level via PCR, using mRNA isolated from meniscus-derived progenitor cells. (F) CD44 molecules were also localized in vitro by fluorescence immunocytochemistry. $(G)$ Total cell's extract were loaded on a gel and stained for coomassie brilliant blue R-250. $(\mathrm{H}+\mathrm{l})$ Membranes were incubated with antibodies against CD29 (H) and CD44 (I), separately. Proteins bands for CD29 and CD44 were visualized after ECL reactions at $135 \mathrm{kDa}$ and $45 \mathrm{kDa}$ respectively. 
Fig. 4

Structural overlaps and differences between mouse and human meniscus (A-B): Histologically, mouse meniscus has some resemblance with human meniscus in terms of vascular (R) and non-vascular (W) parts. However, mouse menisci lack a true superficial (S) zone. (C) The phenotype of human meniscus superficial zone cells is flattened. (D) In contrast, these spindle shape cells are absent in the pseudo-superficial zone of mouse meniscus. (E) Furthermore, the flattened cells in the superficial zone of human meniscus are stained for collagen type I. (F) On the other hand, mouse meniscus stained less for collagen type I in the same region.

References:

Arnoczky SP, Warren RF (1983) The microvasculature of the meniscus and its response to injury. An experimental study in the dog. Am J Sports Med 11: 131-141

Bian L, Zhai DY, Mauck RL, Burdick JA (2011) Coculture of human mesenchymal stem cells and articular chondrocytes reduces hypertrophy and enhances functional properties of engineered cartilage. Tissue Eng Part A 17: 1137-1145

Blagojevic M, Jinks C, Jeffery A, Jordan KP (2010) Risk factors for onset of osteoarthritis of the knee in older adults: a systematic review and meta-analysis. Osteoarthritis and cartilage / OARS, Osteoarthritis Research Society 18: 24-33

Centers for Disease C, Prevention (2010) Prevalence of doctor-diagnosed arthritis and arthritis-attributable activity limitation --- United States, 2007-2009. MMWR Morb Mortal Wkly Rep 59: 1261-1265

Cui X, Hasegawa A, Lotz M, D'Lima D (2012) Structured three-dimensional co-culture of mesenchymal stem cells with meniscus cells promotes meniscal phenotype without hypertrophy. Biotechnol Bioeng 109: 2369-2380

Ferris D, Frisbie D, Kisiday J, Mcllwraith CW (2012) In vivo healing of meniscal lacerations using bone marrow-derived mesenchymal stem cells and fibrin glue. Stem Cells Int 2012: 691605

Furumatsu T, Kanazawa T, Yokoyama Y, Abe N, Ozaki T (2011) Inner meniscus cells maintain higher chondrogenic phenotype compared with outer meniscus cells. Connect Tissue Res 52: 459-465

Hasan J, Fisher J, Ingham E (2013) Current strategies in meniscal regeneration. J Biomed Mater Res B Appl Biomater

Hommen JP, Applegate GR, Del Pizzo W (2007) Meniscus allograft transplantation: tenyear results of cryopreserved allografts. Arthroscopy 23: 388-393 
Katagiri H, Muneta T, Tsuji K, Horie M, Koga H, Ozeki N, Kobayashi E, Sekiya I (2013) Transplantation of aggregates of synovial mesenchymal stem cells regenerates meniscus more effectively in a rat massive meniscal defect. Biochem Biophys Res Commun 435: 603-609

Koelling S, Kruegel J, Irmer M, Path JR, Sadowski B, Miro X, Miosge N (2009) Migratory chondrogenic progenitor cells from repair tissue during the later stages of human osteoarthritis. Cell Stem Cell 4: 324-335

Lawrence RC, Felson DT, Helmick CG, Arnold LM, Choi H, Deyo RA, Gabriel S, Hirsch R, Hochberg MC, Hunder GG, Jordan JM, Katz JN, Kremers HM, Wolfe F, National Arthritis Data W (2008) Estimates of the prevalence of arthritis and other rheumatic conditions in the United States. Part II. Arthritis Rheum 58: 26-35

Mauck RL, Yuan X, Tuan RS (2006) Chondrogenic differentiation and functional maturation of bovine mesenchymal stem cells in long-term agarose culture. Osteoarthritis and cartilage / OARS, Osteoarthritis Research Society 14: 179-189

McDermott ID, Amis AA (2006) The consequences of meniscectomy. J Bone Joint Surg $\mathrm{Br}$ 88: 1549-1556

Muhammad H, Schminke B, Miosge N (2013) Current concepts in stem cell therapy for articular cartilage repair. Expert Opin Biol Ther 13: 541-548

Noble J, Hamblen DL (1975) The pathology of the degenerate meniscus lesion. J Bone Joint Surg Br 57: 180-186

Osawa A, Harner CD, Gharaibeh B, Matsumoto T, Mifune Y, Kopf S, Ingham SJ, Schreiber V, Usas A, Huard J (2013) The use of blood vessel-derived stem cells for meniscal regeneration and repair. Med Sci Sports Exerc 45: 813-823

Pauli C, Grogan SP, Patil S, Otsuki S, Hasegawa A, Koziol J, Lotz MK, D'Lima DD (2011) Macroscopic and histopathologic analysis of human knee menisci in aging and osteoarthritis. Osteoarthritis and cartilage / OARS, Osteoarthritis Research Society 19: 1132-1141

Pelttari K, Winter A, Steck E, Goetzke K, Hennig T, Ochs BG, Aigner T, Richter W (2006) Premature induction of hypertrophy during in vitro chondrogenesis of human mesenchymal stem cells correlates with calcification and vascular invasion after ectopic transplantation in SCID mice. Arthritis Rheum 54: 3254-3266

Proffen BL, McElfresh M, Fleming BC, Murray MM (2012) A comparative anatomical study of the human knee and six animal species. The Knee 19: 493-499

Punwar S, Khan WS (2011) Mesenchymal stem cells and articular cartilage repair: clinical studies and future direction. Open Orthop J 5 Suppl 2: 296-301

Sakaguchi Y, Sekiya I, Yagishita K, Muneta T (2005) Comparison of human stem cells derived from various mesenchymal tissues: superiority of synovium as a cell source. Arthritis Rheum 52: 2521-2529 
Seol D, McCabe DJ, Choe H, Zheng H, Yu Y, Jang K, Walter MW, Lehman AD, Ding L, Buckwalter JA, Martin JA (2012) Chondrogenic progenitor cells respond to cartilage injury. Arthritis Rheum 64: 3626-3637

Shen W, Chen J, Zhu T, Yin Z, Chen X, Chen L, Fang Z, Heng BC, Ji J, Chen W, Ouyang HW (2013) Osteoarthritis prevention through meniscal regeneration induced by intraarticular injection of meniscus stem cells. Stem Cells Dev 22: 2071-2082

Till JE, McCulloch EA (2012) A direct measurement of the radiation sensitivity of normal mouse bone marrow cells. 1961. Radiat Res 178: AV3-7

Walker PS, Erkman MJ (1975) The role of the menisci in force transmission across the knee. Clin Orthop Relat Res: 184-192

Zhang D, Cheriyan T, Martin SD, Gomoll AH, Schmid TM, Spector M (2011) Lubricin distribution in the torn human anterior cruciate ligament and meniscus. Journal of orthopaedic research : official publication of the Orthopaedic Research Society 29: 1916-1922 
Fig. 1
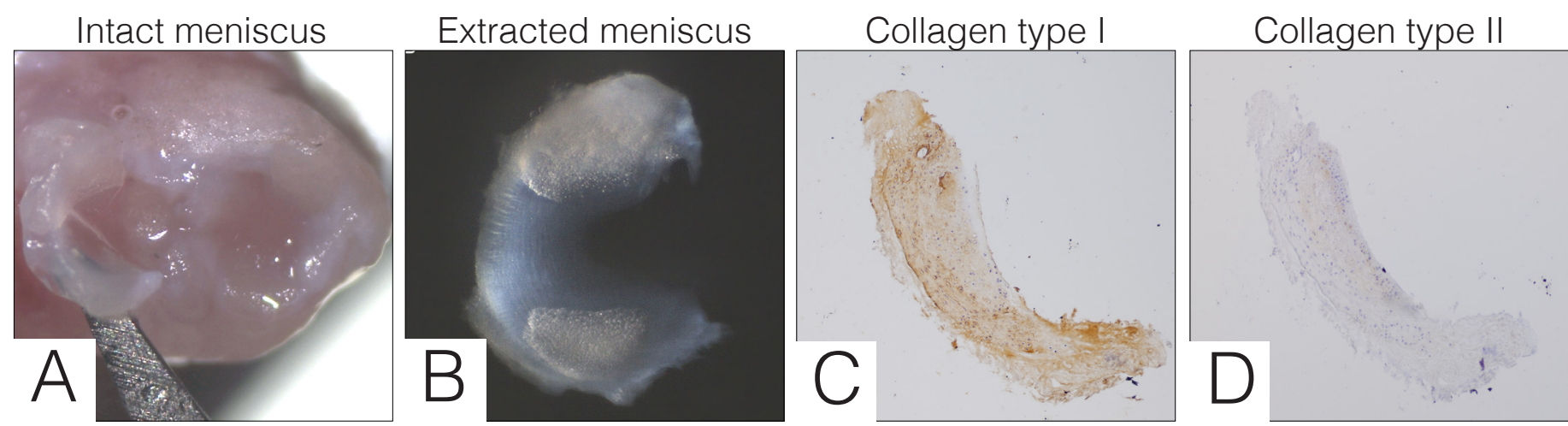

Mouse knee joint

Mouse knee joint section
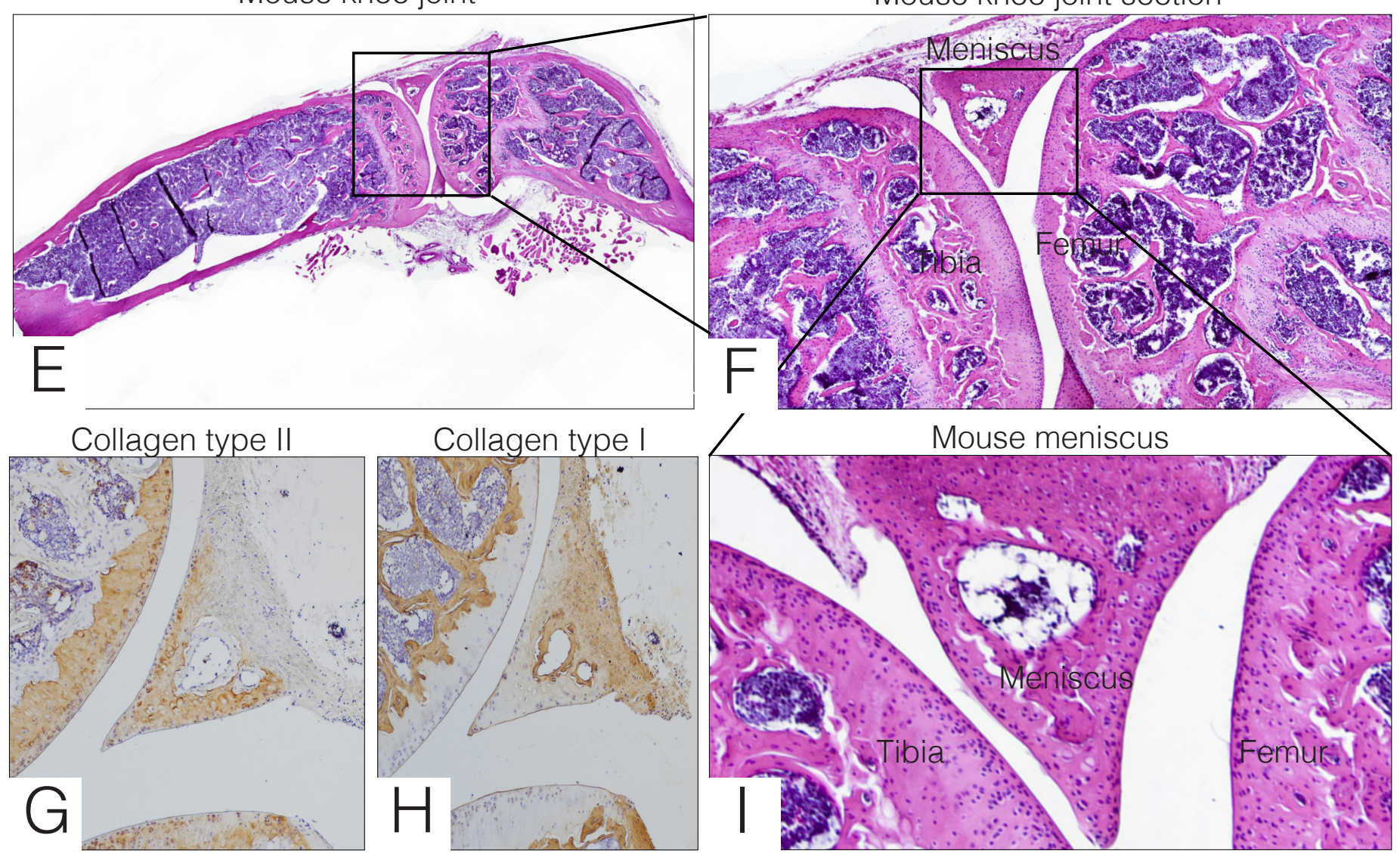
Fig. 2
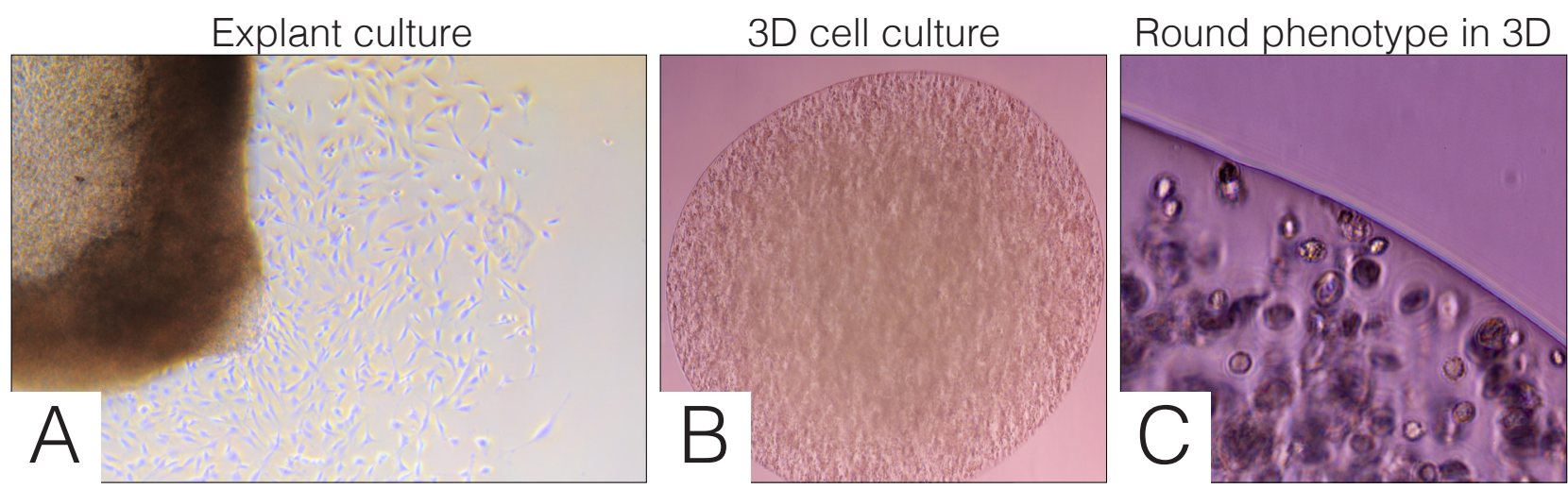

Stem cell markers in vivo and in vitro

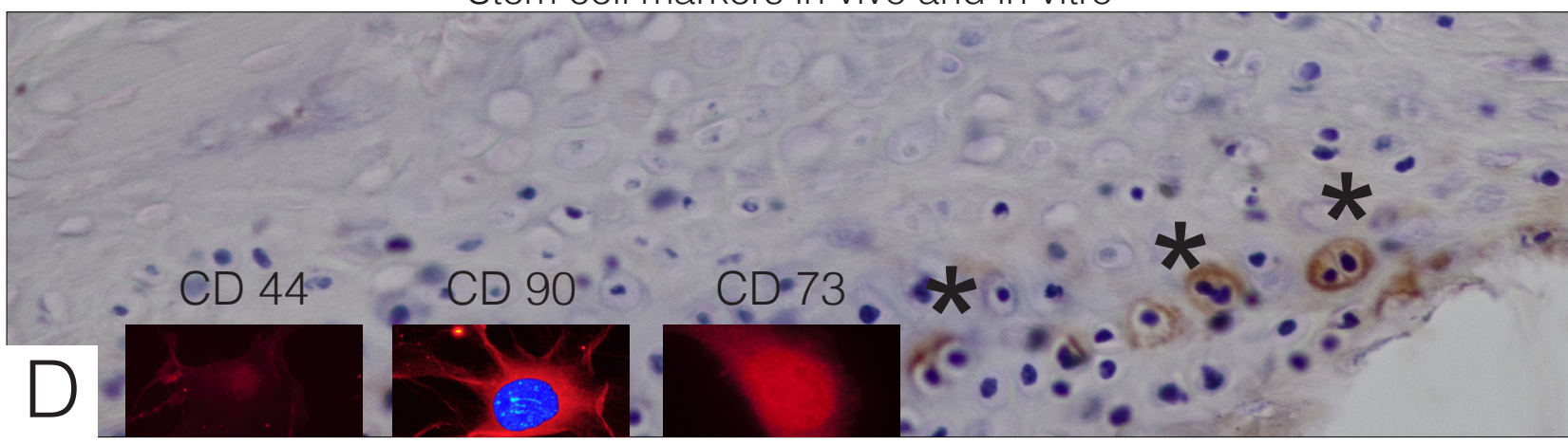

Stem cell marker FACS

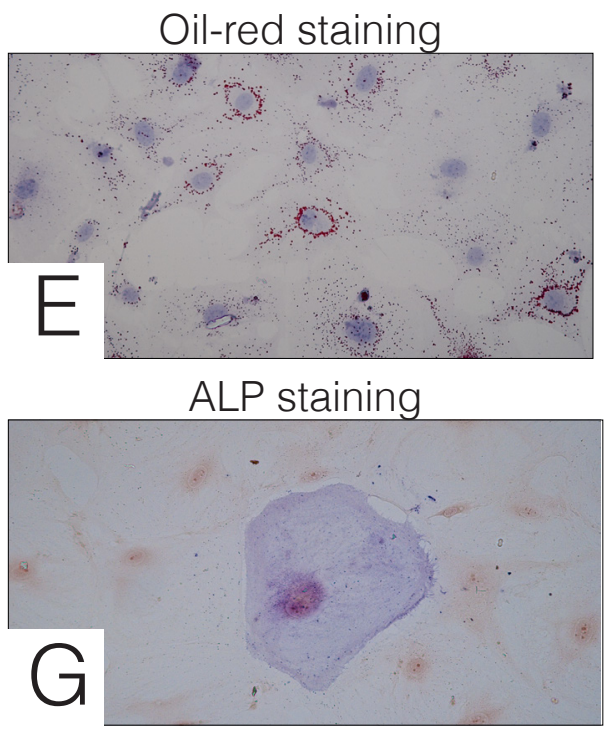

Oil-red positive

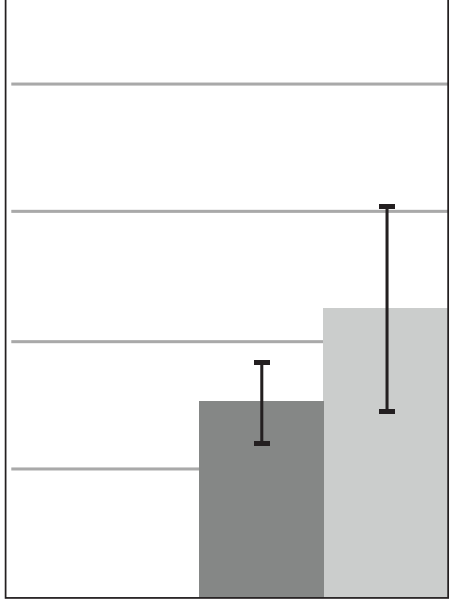

M

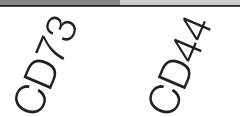

Control oil-red staining
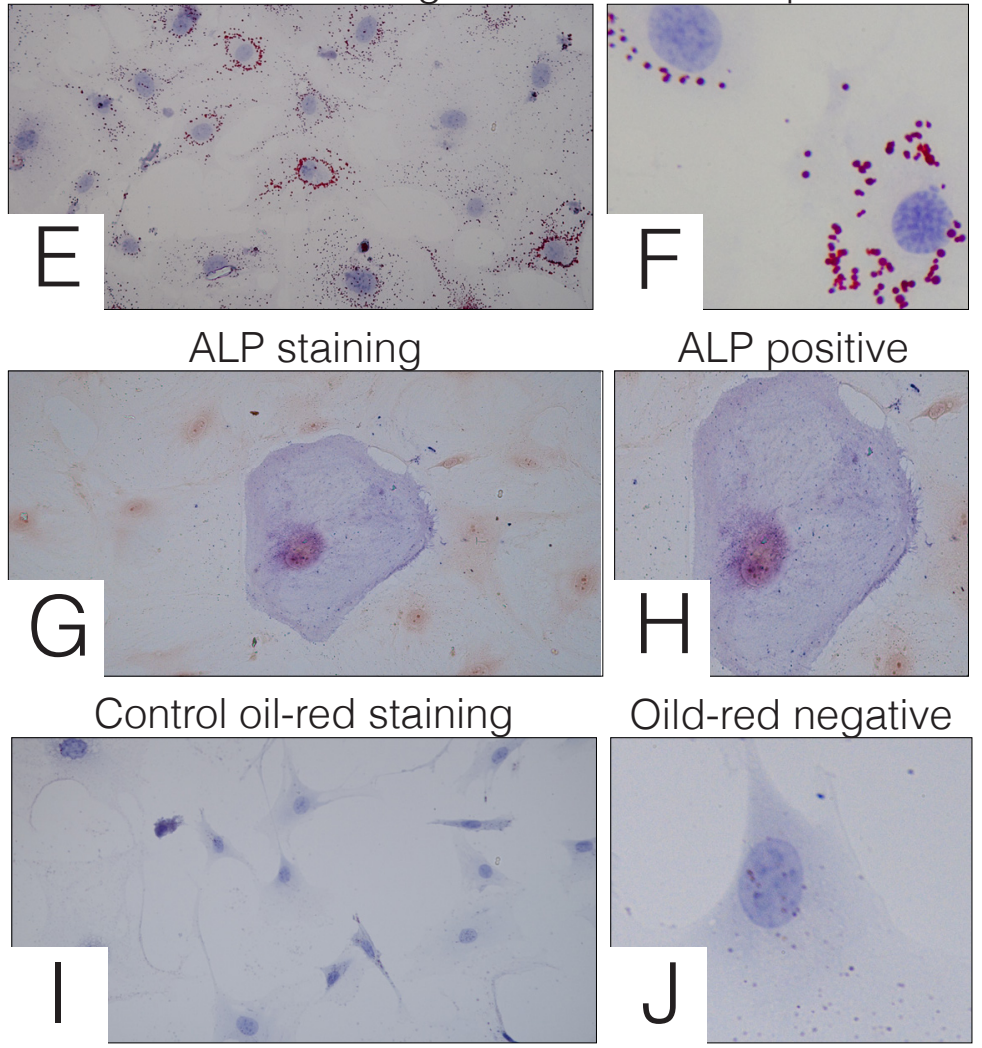

ALP positive

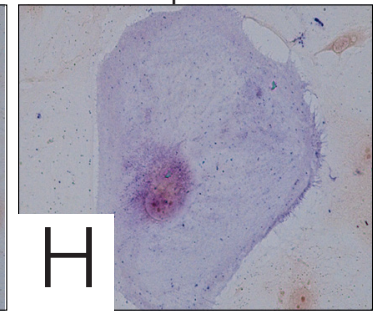

Oild-red negative
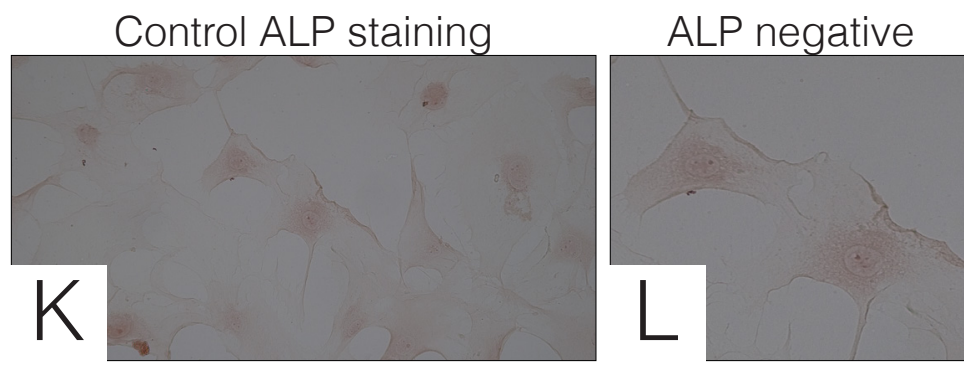
Fig. 3

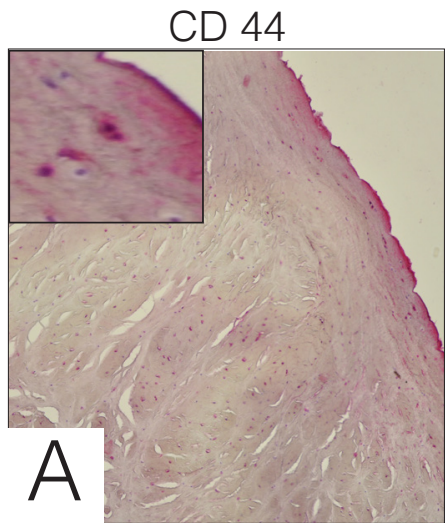

mRNA

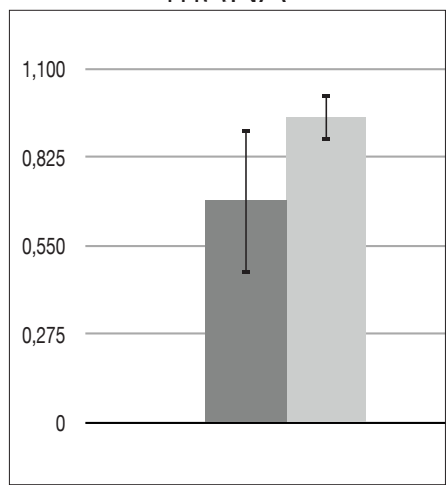

$E 0^{2} 0^{0^{3}}$

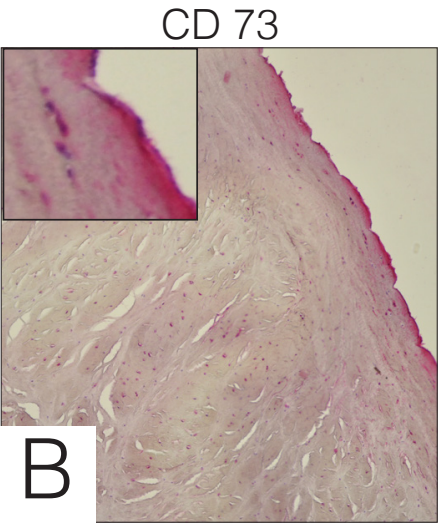

CD 44

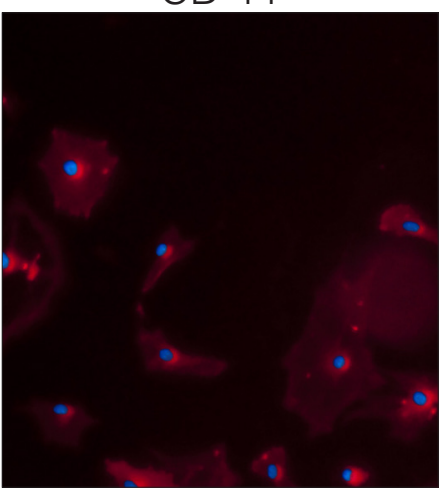

F
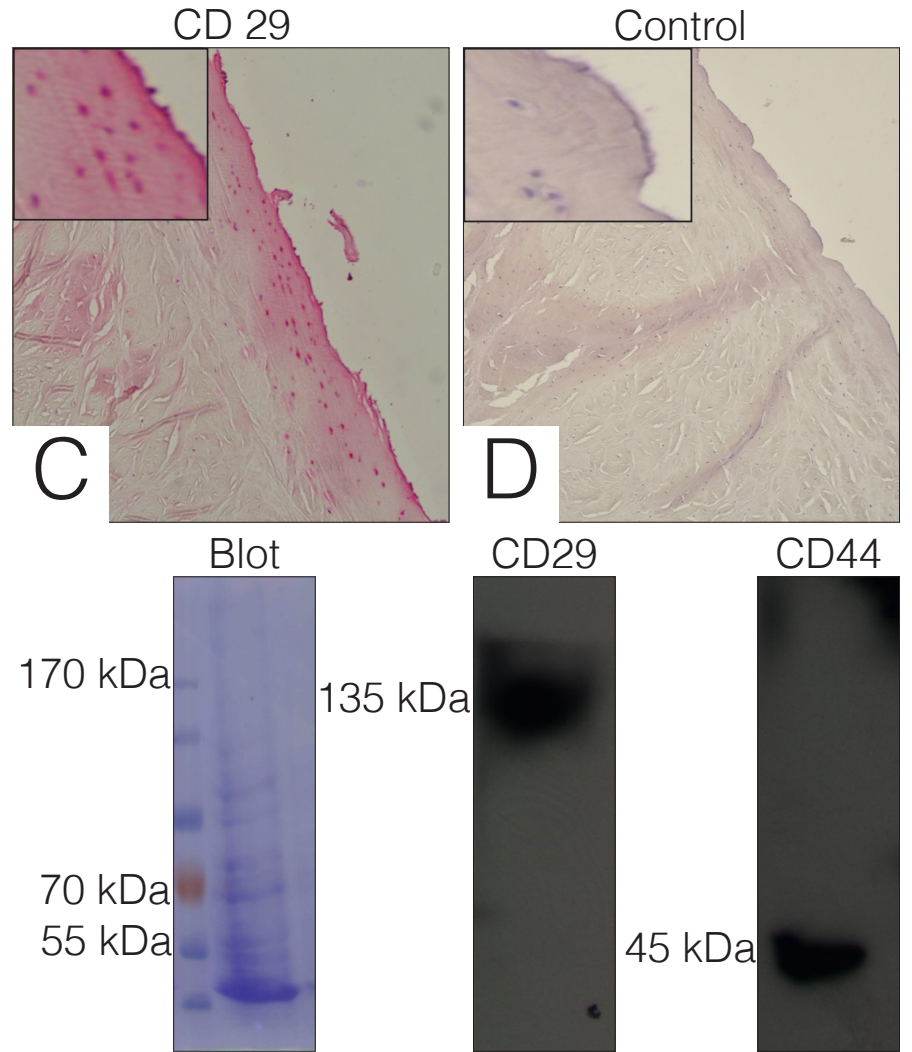

G
$135 \mathrm{kDa}$

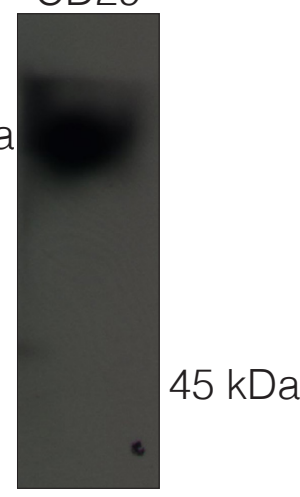

$\mathrm{H}$
CD44

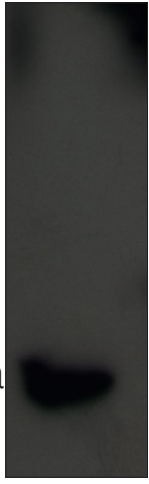

I 
Fig. 4

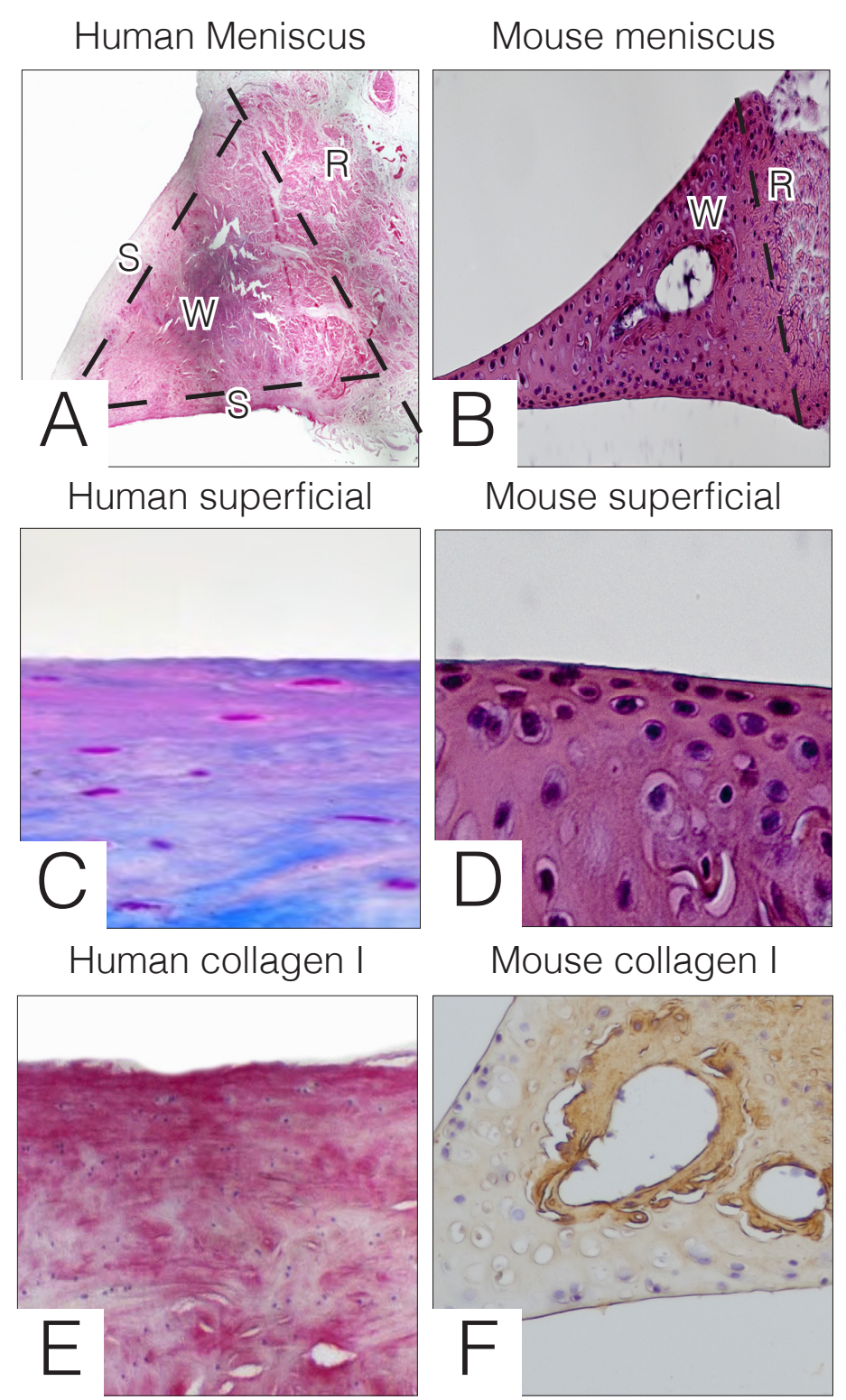




\section{Chapter 6}

Use of chondrogenic progenitor cells in osteoarthritis

Boris Schminke$^{1}$, Nicolai Miosge ${ }^{1 /}$ and Hayat Muhammad ${ }^{1}$

${ }^{1}$ Tissue Regeneration Work Group, Medical Faculty

Department of Prosthodontics, Georg August University

D-37075 Goettingen, Germany

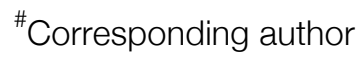

Hayat Muhammad's contribution: Literature search, partially manuscript preparation and image design. 


\title{
Use of Chondrogenic Progenitor Cells in Osteoarthritis
}

\author{
Boris Schminke, ${ }^{1}$ Nicolai Miosge ${ }^{\mathrm{a}, *}$ and \\ Hayat Muhammad ${ }^{1}$
}

\begin{abstract}
It is thought that the general increase in life expectancy will make osteoarthritis the fourth leading cause of disability by the year 2020 . Even though the pathogenesis of idiopathic osteoarthritis has not been fully elucidated, the main features of the disease process are the altered interactions between the chondrocytes and their surrounding extracellular matrix. In the course of these disturbances fibroblast-like chondrocytes take part in tissue regeneration especially in advanced stages of osteoarthritis. However, only fibrocartilaginous or scar tissue, since only collagen type I, and not collagen type II, typical for healthy cartilage, is synthesized. It remains a great challenge to enhance the regeneration potential of hyaline cartilage tissue. Tissue degeneration overrides the generally limited self-renewal capacity of this tissue.
\end{abstract}

\footnotetext{
Tissue regeneration work group, Medical Faculty, Department of Prosthodontics, Georg August University, Goettingen, D-37075, Germany.

${ }^{\text {a }}$ E-mail: nmiosge@gwdg.de

${ }^{*}$ Corresponding author

List of abbreviations given at the end of the text.
} 
Adult mesenchymal stem cells, which are thought to be capable of repairing injured tissue can be differentiated into chondrocyte-like cells in vitro. During embryonic development, some cells of the inner cell mass will develop into the mesoderm. This will be the founder of the mesenchymal cells in connective tissues of adult life, such as bone, tendon, muscle, and cartilage. Some of these embryonic mesenchymal cells are believed not to differentiate, but reside in each of the tissues. These are now collectively described as adult mesenchymal stem cells, which are thought to be capable of repairing injured tissue. We found that repair tissue from human articular cartilage during the late stages of osteoarthritis harbors a unique progenitor cell population, termed chondrogenic progenitor cells (CPC). These exhibit stem cell characteristics together with a high chondrogenic potential. They will be relevant in the development of novel therapeutic regenerative approach for a progenitor cell-based therapy of late stages of OA.

\section{Introduction}

Osteoarthritis (OA) is a chronic and mainly degenerative joint disease. Degeneration is progressive and the loss of articular cartilage finally leads to the eburnation of the subchondral bone (Fig. 13.1). The process is accompanied by an inflammatory synovial reaction (Poole et al. 1993). $\mathrm{OA}$ is the most common musculoskeletal disease in the elderly, according to Reginster (2002) up to 1.75 million people alone in England and Wales suffer from symptomatic OA. However, the number of asymptomatic cases is estimated to be much higher. There is a strong association between its prevalence and increasing age, since up to 20 percent of the population over $60 \mathrm{yr}$ of age show signs of OA (Haq et al. 2003). The severity of OA also increases indefinitely with age and up to now the condition is not reversible (Woolf and Pfleger 2003). As OA often remains asymptomatic until late in the disease progress and early markers as reliable tools of diagnosis are still lacking up to now, therefore, total knee replacement is the ultimate therapeutic intervention. This means that important parts of health care resources have to be spent on coping with this disease (Reginster 2002). The general increase in life expectancy and the resulting aging populations are expected to make OA the fourth leading cause of disability by the year 2020 (Woolf and Pfleger 2003). This warrants the further elucidation of the pathogenesis of OA with the final goal of gaining insight into the disease processes to render a cell biological therapy possible and within reach. Regenerative medicine and tissue engineering approaches are being investigated and developed further. Here, we review the current knowledge on progenitor cells and their possible usage in future therapies of osteoarthritis. 

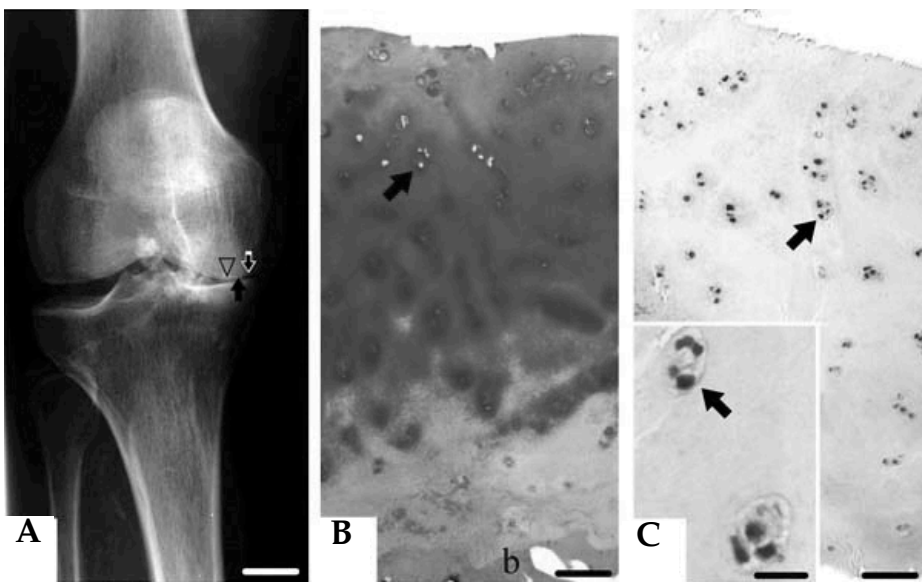

Figure 13.1 Adapted from Bock et al. 2001 (with permission from the publisher). (A) Radiograph of a patient with grade IV OA. Areas from which the cartilage samples were taken are marked (black arrow $=$ main defect, open triangle=adjoining area and black-on-white arrow $=$ macroscopically intact), $b a r=2 \mathrm{~cm}$. (B) Alcian blue staining of a cartilage sample from the macroscopically intact area; note that chondrocytes are organized in clusters (black arrow), bar $=50 \mu \mathrm{m}$. (C) Light microscopic in situ hybridization in a sample similar to (B), intracellular staining for biglycan mRNA (Black arrows), bar=50 $\mu \mathrm{m}$, inset: higher magnification of two cell clusters.

Color image of this figure appears in the color plate section at the end of the book.

\section{Articular Cartilage: Chondrocytes and Matrix Composition in Health and Disease}

Normal, healthy articular cartilage is a highly specialized and uniquely designed tissue, which covers the articulating ends of long bones (Kuettner 1992). It is an avascular, aneural and alymphatic tissue. The resilience, integrity and function of articular cartilage all depend on the composition of the abundant extracellular matrix (ECM) synthesized by the single cell type found in this tissue, the chondrocytes. These are responsible for the secretion and maintenance of the ECM of articular cartilage (Kuettner 1992). The abundant extracellular matrix of articular cartilage is composed of two major elements: the collagens and the proteoglycans. Normal articular cartilage contains types II, III, VI, IX, X, XI, XII and XIV collagens, the most abundant being collagen type II (Mayne and Brewton 1993). Collagens type II, IX and XI form fibrillar alloys with type XI collagen as core and type IX collagen on the outside possibly limiting the fiber diameter (Kuettner 1992). In addition, the proteoglycans, a heterogeneous group of proteins, consisting of a central core protein substituted with one or more glycosaminoglycan side chains constitute the other major extracellular matrix components. A few good examples would be, first of all, aggrecan, the large cartilage 
matrix proteoglycan responsible for cartilage tissue maintenance together with several other small proteoglycans, which are also important for its function, such as decorin, biglycan and fibromodulin.

A disturbed cell-matrix relationship lies at the center of the pathogenesis of OA (Poole 1999). The degradation of the tissue by matrix metalloproteases is underlined by a loss of the main proteoglycan, aggrecan, collagen fiber fibrillation and surface splits (Poole 1999; Martel-Pelletier 1999). However, this tissue degeneration is intermingled with regeneration efforts (Sandell and Aigner 2001), which might possibly be seen in the occurrence of chondrocyte clusters and are certainly seen in the appearance of fibrocartilaginous tissue with a more fibrillar matrix and a newly emerging cell type (Bock et al. 2001; Poole 1999). These cells were initially identified and described at the ultrastructural level and named elongated secretory type 2 cells (Fig. 13.2) and had an irregular shape with a prominent rough endoplasmic reticulum (Kouri et al. 1996). We called them fibroblastlike chondrocytes, which build the fibrocartilagenous tissue at the more advanced stages of OA (Sandell and Aigner 2001; Miosge et al. 1998). This repair tissue is mainly composed of collagen type I, whereas physiological articular cartilage reveals only collagen type II. Collagen type I protein has been detected at the light microscopic level in osteoarthritic cartilage with the help of immunohistochemistry. Recently, we identified a subpopulation of these cells as chondrogenic progenitor cells (Koelling et al. 2009; Koelling et al. 2009; Koelling and Miosge 2010).

\section{From Embryonic Stem Cells to Mesenchymal Cells and Adult Progenitor Cells}

The original stem cells reside in the inner cell mass of the embryo proper at the stage of the blastocyst. Before this developmental stage, one could argue, that every cell of the morula is a stem cell. Obviously, cells of the morula are pluripotent and capable of developing into each of the three germ layers that will later, during the course of embryogenesis, develop into the specific tissues to form the organs (O'Rahilly et al. 1981). During early embryonic development, some of the embryonic stem cells of the inner cell mass of the blastocyst will turn into ectoderm. Derivatives of this germ layer will, for example, develop into skin and brain-tissues devoted to connecting organisms to the exterior world. Some will turn into endoderm. This germ layer will mainly form internal organs, for example, the gut and the liver. The third germ layer, the mesoderm, will develop from the ectoderm, known as the first ectoderm-mesenchyme transition. This mesoderm will be the founder of the mesenchymal cells later found in the connective tissues. In adult life, these tissues, such as bone, tendon, 

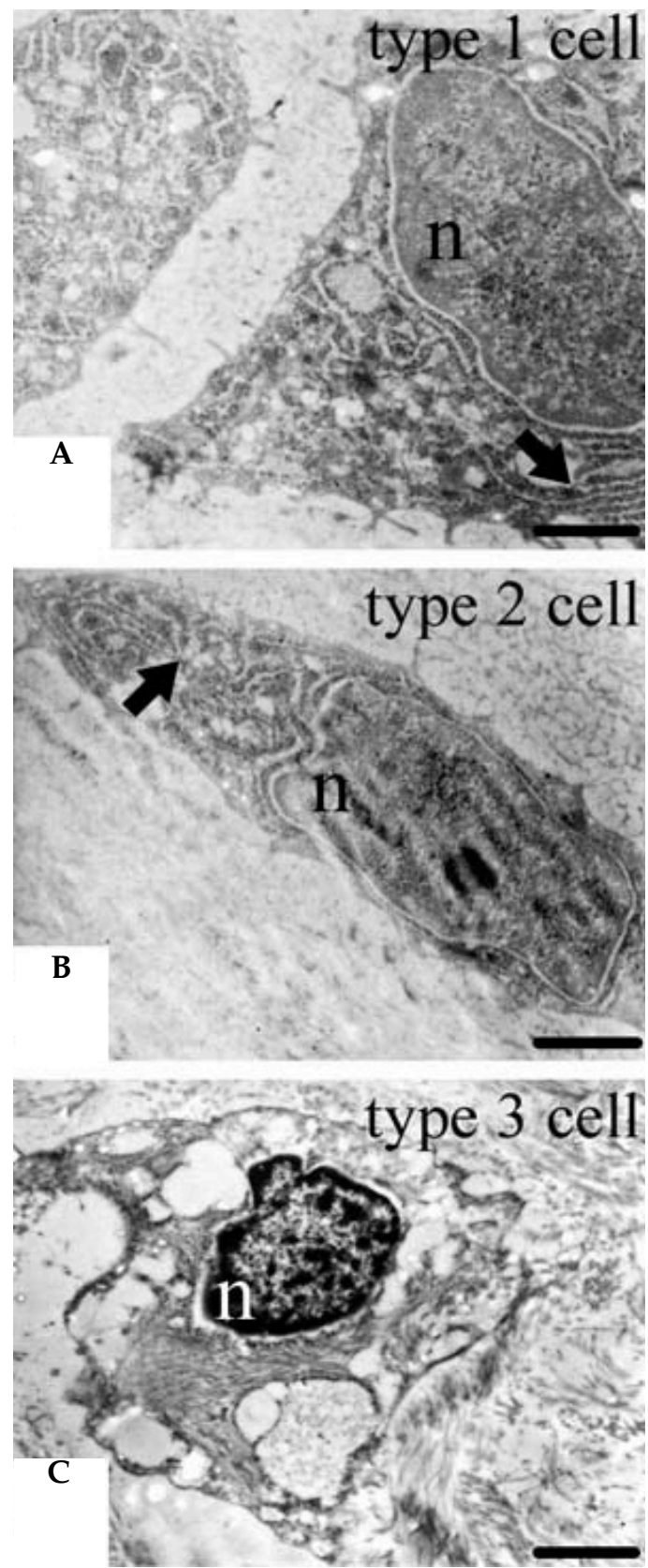

Figure 13.2 Adapted from Bock et al. 2001 (with permission from the publisher), Electron micrographs of the three chondrocyte types: (A) normal, (B) fibroblast-like, (C) degenerating $\mathrm{n}=$ nucleus, black arrows $=$ endoplasmic reticulum, bars $=0,7 \mu \mathrm{m}$. 
muscle, and cartilage, are the building blocks of the skeletal system. Adult mesenchymal cells exhibit a profound plasticity. Adult differentiated cells like chondrocytes, which were kept in culture on plastic dishes for prolonged periods of time, dedifferentiate to an extent that allows for the reoccurrence of characteristics of stem cells (Dell'Accio et al. 2003). In vitro, adult skin fibroblasts have also been turned into stem cell-like cells with the help of the transfection of the transcription factors oct3/4, sox2, c-myc and klf4 (Takahashi and Yamanaka et al. 2006). Mesenchymal stem cells are believed to be capable to regenerate diseased tissues. We would prefer to call them mesenchymal progenitor cells, as all those cells described in vivo and in vitro are migratory cells that have left their original stem cell niche and, therefore, belong to the transient amplifying pool of cells. Per definitionem, the stem cell resides in its niche composed of adjacent, more differentiated, cells and extracellular matrix molecules (Fuchs et al. 2004).

Friedenstein et al. (1970) was the first to describe fibroblast colonies derived from guinea-pig bone marrow and this paved the way to the further characterization of bone marrow stroma cells. To date, various populations of bone marrow stroma cells have been described as stem cells and differentiated into cartilage tissue in vivo and in vitro. Due to space limitation, we can only highlight a few of these studies here. Johnstone et al. (1998) described rabbit mesenchymal cells differentiated in vitro into a tissue staining positive for collagen type II protein. Mackay et al. (1998) also described the chondrogenic differentiation of mesenchymal stem cells derived from bone marrow to produce a chondrocyte-like extracellular matrix in pellet culture. Micro-mass culture of human bone marrow stromal cells with the addition of the chondrogenic mediators BMP-6 and TGFß 3 resulted in the formation of cartilage-like tissue in vitro. Gronthos et al. (2003) have also applied bone marrow derived mesenchymal stem cells partially characterized by their expression of STRO-1. The other marker related to stem cells that this group applied is CD106 or VCAM-1, which is an adhesion molecule, is also found on endothelial cells. Chondrogenesis of these STRO- $1^{+} / \mathrm{CD}_{106}{ }^{+}$cells was proven by the detection of mRNA for collagen type II, type $\mathrm{X}$ and aggrecan.

Stem cells from tissue sources other than bone marrow have also been described. Synovia-derived stem cells and cells isolated from the synovial fluid can be differentiated into cartilage-like tissue. The Hoffa fat pad in the knee joint has been described as the origin of stem cells driven into the chondrocyte lineage in vitro (English et al. 2007).

Up until now, only one study in a goat animal model has described the use of mesenchymal stem cells to treat an osteoarthritic defect. In this case, intra-articular injections of mesenchymal stem cells resulted in minor improvement of the disease process. However, the cells migrated to all of the tissues of the knee, except the cartilage tissue itself (Murphy et al. 2003). 


\section{Chondrogenic Progenitor Cells for Cartilage Repair}

Especially in connective tissues, adult stem cell-like cells have been long known to be responsible for tissue repair after injury. In muscle, stellate cells are found and the broken bone heals via activation of mesenchymal cells derived from the inner layers of the periost, the connective tissue surrounding each bone. There is evidence that mesenchymal cells characterized by their surface antigens are found in osteoarthritic cartilage tissue. Alsalameh et al. (2004) isolated $\mathrm{CD}_{105}{ }^{+}$and $\mathrm{CD} 166^{+}$cells from osteoarthritic cartilage tissue by enzymatic digestion and drove them into cartilage-like tissue with the help of micro-mass culture in vitro. In addition Fickert et al. (2004) isolated cells, this time positive for CD9, CD90 and CD166 and were able to demonstrate their differentiation into such a tissue. Moreover, microfracture and Pridie drilling to open the bone marrow underneath the cartilage defect are still used as a therapeutic option and result in a fibrocartilaginous repair tissue. This repair tissue is thought to originate from migrating mesenchymal cells (Fig. 13.3). However this regeneration tissue exhibits

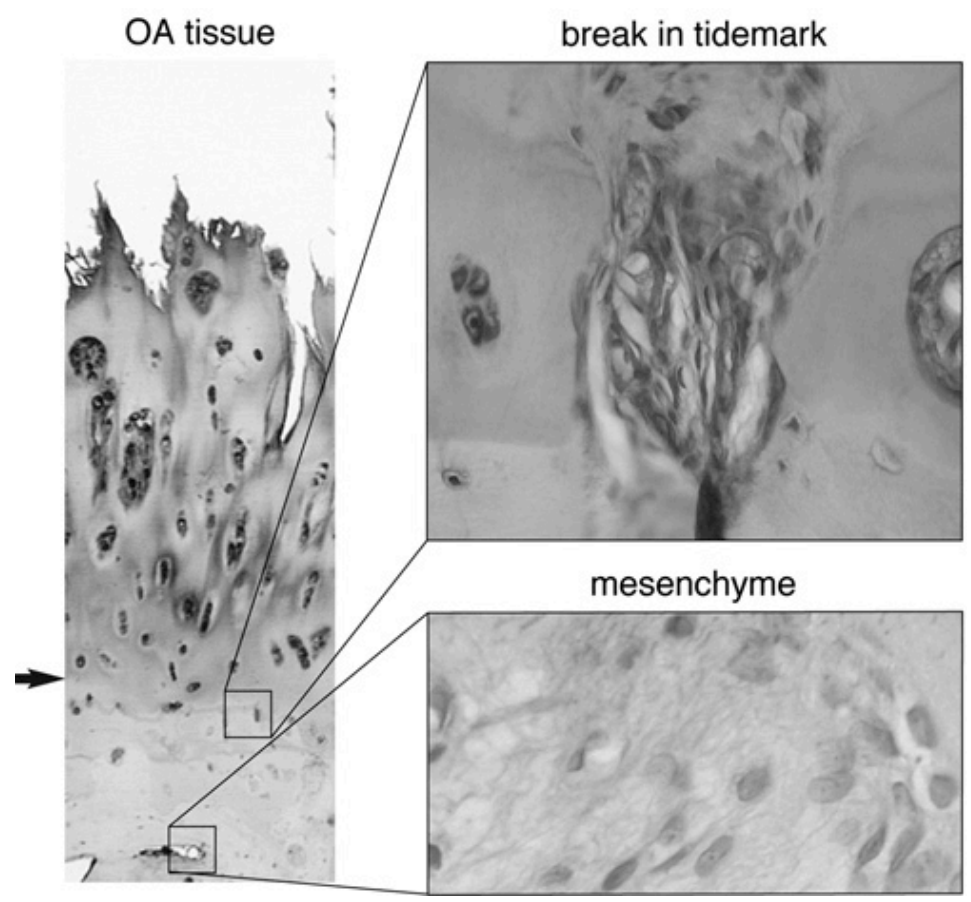

Figure 13.3 The diseased cartilage tissue exhibits deep surface fissures, chondrocytes in clusters and breaks in the tidemark (arrow), taken from Koelling et al. 2009 (with permission from the publisher).

Color image of this figure appears in the color plate section at the end of the book. 
less resistance to mechanical stress and is composed mainly of collagen type I, which is not typically present in healthy articular cartilage tissue (Miosge et al. 1998). Finally, postnatal stem cells have been identified in the superficial zone of healthy bovine cartilage believed to be responsible for the appositional growth of the joint surface (Dowthwaite et al. 2004).

\section{Progenitor Cells in Repair Tissue of Late-stage Osteoarthritis}

Physiological repair mechanisms of diseased hyaline cartilage tissues are sparse and overridden by matrix destruction resulting in less functional fibrocartilaginous, collagen type I-rich scar tissue (Koelling and Miosge 2009). Despite the evidence that stem cells might be involved in regeneration activities seen in osteoarthritis, no studies to date have identified an already committed chondrogenic progenitor cell population in late-stage osteoarthritis. We identified migratory cells derived from repair tissue of late-stage osteoarthritis (Fig. 13.4) which possess a high chondrogenic potential and progenitor cell characteristics. We called them

migration through tidemark

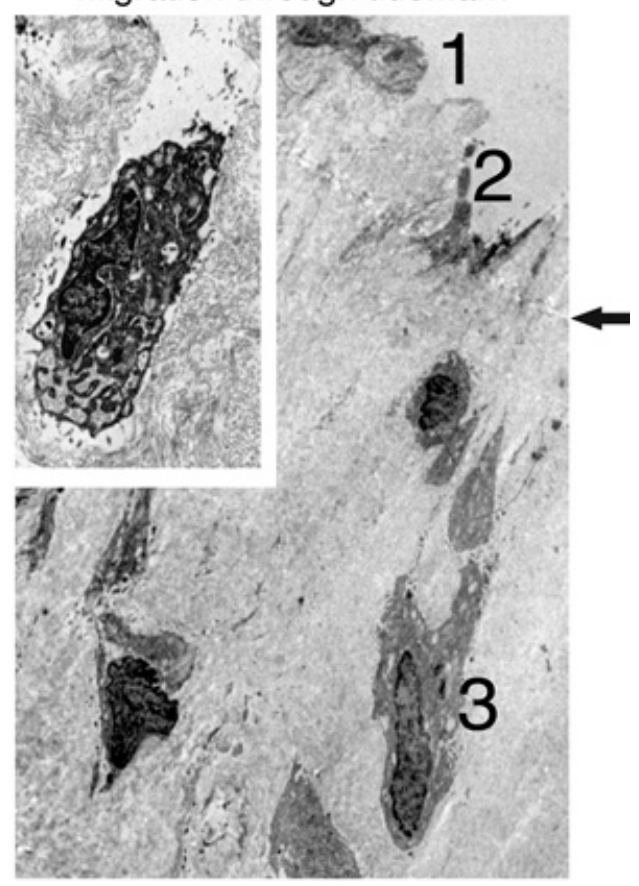

Figure 13.4 A brake in the tidemark with mesenchymal tissue and blood vessels entering the cartilage tissue. Via this route, progenitor cells from the bone marrow enter the diseased tissue, taken from Koelling et al. 2009 (with permission of the publisher). 
chondrogenic progenitor cells (Koelling et al. 2009). These cells possess a multipotent differentiation capacity especially towards the chondrogenic lineage, as well as a migratory potential (Fig. 13.5) and furthermore they

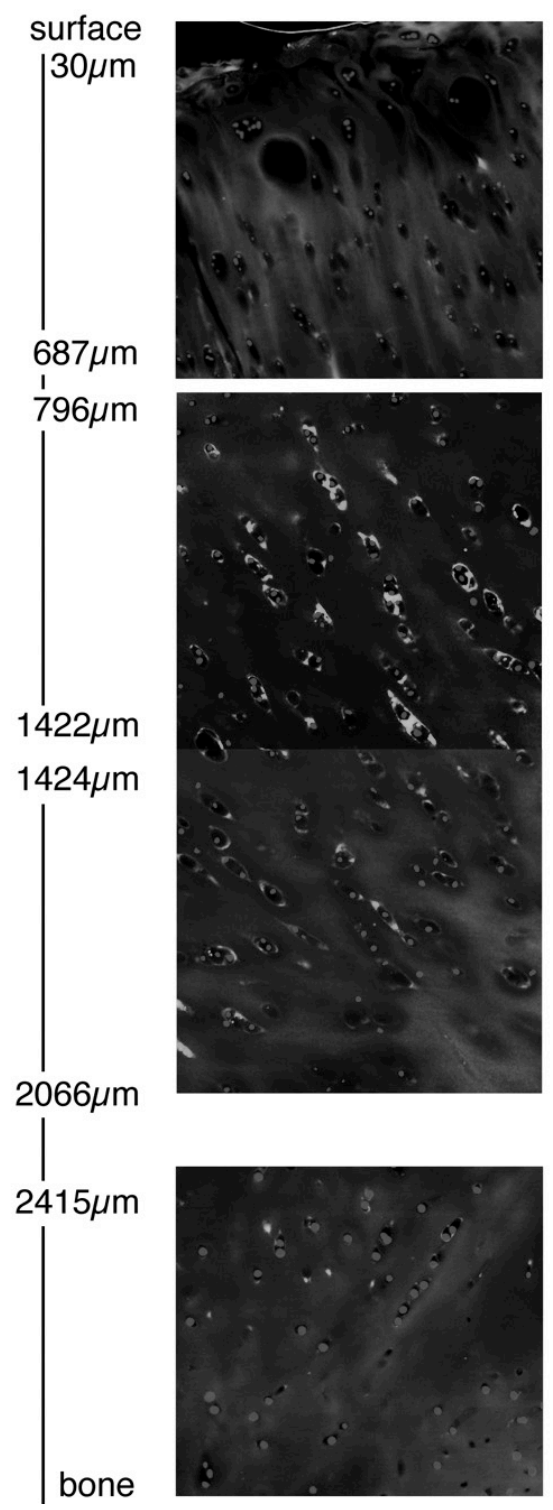

Figure 13.5 Chondrogenic progenitor cells transfected with green fluorescent protein migrate into osteoarthritic tissue ex vivo. These cells are found approx. $1400 \mu \mathrm{m}$ deep in the tissue after $2 \mathrm{~d}$, taken from Koelling et al. 2009 (with permission of the publisher).

Color image of this figure appears in the color plate section at the end of the book. 
also populate diseased tissue in vivo (Fig. 13.6). Because these cells show heterogeneity in these properties and because of their migratory potential, we prefer to call them chondrogenic progenitor cells. Furthermore, with the help of RNA knock-down, we have shown that sox-9 and runt-related transcription factor 2 (runx-2) play a central role in the chondrogenic differentiation process of these cells that are also influenced by mediators from the extracellular matrix. These CPCs are an ideal starting point for a cell biological regenerative therapy of osteoarthritis.

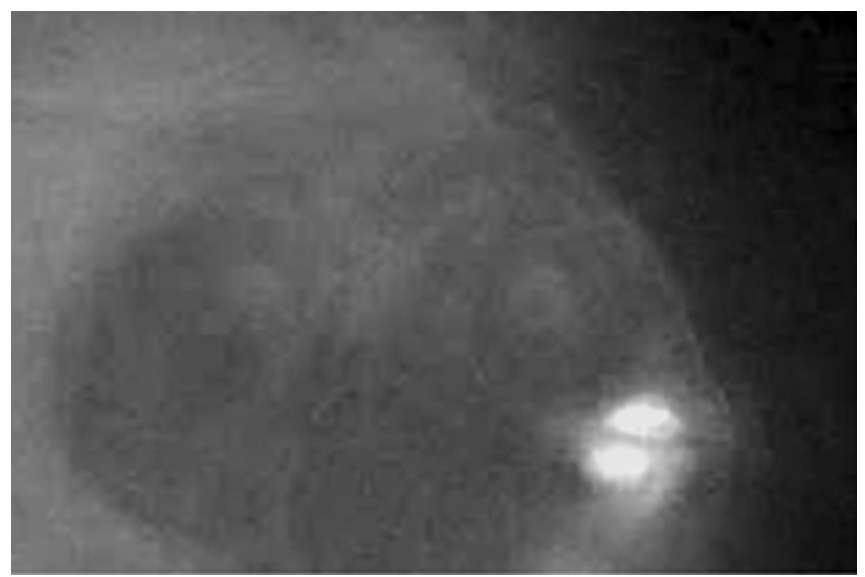

Figure 13.6 Cells with marker proteins related to stem cells are also found in vivo in osteoarthritic cartilage tissue. Here two cells in a cluster are positive for CD29 and CD73, taken from Koelling et al. 2009 (with permission of the publisher).

Color image of this figure appears in the color plate section at the end of the book.

\section{Future Perspectives of a Regenerative Therapy of Osteoarthritis}

There are key limitations inherent to any cell biological therapy of osteoarthritic defects that have to be overcome before a regenerative therapy with progenitor cells will be applicable. First, it has to be shown that these cells can be manipulated to enhance their chondrogenic potential and that it remains present over a long time. The cells present in osteoarthritic tissue are not able to alter the disease process. Their physiological repair capacity is not sufficient. Therefore, it remains to be seen if these cells will produce an extracellular matrix that results in a repair tissue with a higher physical resistance to mechanical stress than the fibrocartilaginous tissue developed during the course of the disease. Finding the optimal conditions to manipulate such cells will be crucial for the development of a cell biological therapy for the treatment of osteoarthritis. Resident cells that 
are already used as a physiological response to the cell biological stimuli of the cartilage tissue, the tissue they are supposed to repair, may be more sufficient than cells derived from a totally different source such as adipose tissue stem cells. Furthermore, stem cells have been shown to gradually lose their differentiation potential with age. Mesenchymal stem cells from patients with osteoarthritis exhibited a reduced potential for differentiation towards a cartilage-like tissue (Murphy et al. 2002). Thus, a new concept sees mesenchymal stem cells as a therapeutic means to positively influence the microenvironment of the stem cells already present in the diseased tissue and to direct those cells in their regeneration activities (Caplan 2007). This is derived from results demonstrating a positive immunomodulatory effect of mesenchymal stem cells, even in allogenic transplantations (Wolbank et al. 2007). However, there is also the possibility that stem cells found in osteoarthritic tissue are responsible for the disease process. Before a cell biological therapy of osteoarthritis becomes a clinical reality, numerous scientific questions remain to be addressed in vitro. However, the crosstalk of these transcription factors mediating pluripotency remains to be elucidated, to gain insight into molecular mechanisms of reversibility of commitment and consecutive plasticity in the cells of the transient amplifying pool. It is well possible, that the transcription factors named above are also important for CPCs and might enhance their multipotency. One of the shortfalls of stem cell therapy is that the stemness of true stem cells is altered, as soon, as it is removed from its niche (Fuchs et al. 2004) and that these cells are the transient amplifying pool already committed towards terminal differentiation (Fuchs 2009). Furthermore, the in vitro-cultivation of these cells further alters their cell biological properties. Therefore, understanding of the underlying mechanisms that govern stemness, multipotency and differentiation is essential for all future stem cell therapy approaches.

\section{Dictionary of Key Terms}

- Osteoarthritis $(O A)$ : OA is mainly degenerative joint disease. Cartilage plays an important role in the joints mechanics for painless and frictionless motion. Loss of the cartilage in the joints permits direct bone to bone exposure which leads to pain, inflammation and ultimately in the loss of the joint.

- Sox9: Sox9 is a transcription factor, DNA binding protein and essential for chondrogenesis. Sox9 is directly involved in the regulation of type II collagen gene, which is the major components in extra cellular matrix (ECM) of hyaline cartilage. Sox9 is considered as master regulator and as a key player during chondrocyte differentiation.

- Runx2: Runx2 is mainly involved in osteogenesis and cartilage development. Runx2 is a transcription factor and acts as "main/master 
switch" in regulating many other genes that are involved in promoting the osteogenic characters in cells as in osteoblasts.

- Mesenchymal stem cells (MSCs): MSCs mostly reside in bone marrow, basically mesenchyme is embroyonic connective tissue that derived from the mesoderm. MSCs differentiates into multiple cell types including adipocytes, chondrocytes, and cell of the osteoblastic lineage. In short, MSCs are capable to regenerate the diseased tissue.

- Progenitor stem cells: Progenitor stem cells behave like stem cells and have the ability to differentiate into specific types of target cell. The most prominent and clear difference between progenitor cells and stem cells is that the stem cells can divide indefinitely and are open to choose their differentiation direction while progenitor cells are already determinated to a more narrow cell fate decision for example cells of the skeletal system and have a limit number of replication cycles.

\section{Summary Points}

- Osteoarthritis (OA) is such a wide spread complication associated with aging that it is expected to become the fourth most frequent cause of disability by the year 2020 and cartilage regeneration becomes a challenge for the regenerative and bio-engineering community.

- Osteoarthitis is a mainly degenerative joint disease. Eventual loss of cartilage in the joint uncovers the bone and allows direct bone to bone contact. The frictional moment of joint damaging the bone and ultimately results in pain and inflammation. This mechanical disability arises due to destruction and vanishing of extracellular matrix proteins particularly collagen type II and proteoglycans in cartilaginous tissue.

- Chondrogeinc progenitor cells (CPCs) are present in the late stages of human OA. They migrate from the fibrocartilaginous tissue in vitro.

- CPCs exhibit stem cell characteristics such clonogencity, multipotency and migratory activity.

- CPCs have tremendous ability towards chondrogensis and this chondrogenic potential can be enhanced by knocking down (using siRNA) Runx2 (an osteogenic protein) or by over expression of sox9 (a chondrogenic protein).

\section{Acknowledgements}

We would like to apologize to all the colleagues whose work we could not mention due to space limitations. This work was supported by the German Research Council (DFG) and the Medical Faculty in Goettingen. 


\section{List of Abbreviations}

$\begin{array}{lll}\text { OA } & : & \text { osteoarthritis } \\ \text { ECM } & : & \text { extracellular matrix } \\ \text { CPC } & : & \text { chondrogenic progenitor cells } \\ \text { MSC } & : & \text { mesenchymal stem cells }\end{array}$

\section{Transcription factors}

\begin{tabular}{|c|c|c|}
\hline oct3 $/ 4$ & : & octamer-binding transcription factor $3 / 4$ \\
\hline sox2 & & SRY (sex determining region Y)-box 2 \\
\hline c-myc & & cellular myelocytomatosis oncogene \\
\hline klf4 & & Krüppel like factor 4 \\
\hline STRO-1 & & $\begin{array}{l}\text { antigen identified by Simmons and Torok-Storb for } \\
\text { stem cells }\end{array}$ \\
\hline \multirow{3}{*}{\multicolumn{2}{|c|}{$\begin{array}{l}\text { CD106, CD9, : } \\
\text { CD90 and } \\
\text { CD166, CD105 }\end{array}$}} & cluster of differentiation, so called stem cell markers \\
\hline & & \\
\hline & & \\
\hline VCAM-1 & : & vascular cell adhesion molecule 1 \\
\hline BMP-6 & : & bone morphogenetic protein 6 \\
\hline $\mathrm{TGFB}_{3}$ & : & transforming growth factor beta 3 \\
\hline Runx-2 & & runt-related transcription factor 2 \\
\hline Sox9 & & SRY (sex determining region Y)-box 9 \\
\hline
\end{tabular}

\section{References}

Alsalameh, S., R. Amin, T. Gemba and M. Lotz. 2004. Identification of mesenchymal progenitor cells in normal and osteoarthritic human articular cartilage. Arthritis Rheum. 50(5): 1522-1532.

Bock, H.C., P. Micheali, C. Bode, W. Schultz, H. Kresse, R. Herken and N. Miosge. 2001. The small proteoglycans decorin and biglycan in human articular cartilage of late-stage osteoarthritis. Osteoarthritis and Cartilage. 9: 654-663.

Caplan, A.I. 2007. Adult mesenchymal stem cells for tissue engineering versus regenerative medicine. J Cell Physiol. 213(2): 341-347.

Dell'Accio, F., J. Vanlauwe, J. Bellemans, J. Neys, C. De Bari and F.P. Luyten. 2003. Expanded phenotypically stable chondrocytes persist in the repair tissue and contribute to cartilage matrix formation and structural integration in a goat model of autologous chondrocyte implantation. J Orthop Res. 21(1): 123-131.

Dowthwaite, G.P., J.C. Bishop, S.N. Redman, I.M. Khan, P. Rooney, D.J. Evans, L. Haughton, Z. Bayram, S. Boyer, B. Thomson et al. 2004. The surface of articular cartilage contains a progenitor cell population. J Cell Sci. 117(Pt 6): 889-897.

English, A., E.A. Jones, D. Corscadden, K. Henshaw, T. Chapman, P. Emery and D. McGonagle. 2007. A comparative assessment of cartilage and joint fat pad as a potential source of cells for autologous therapy development in knee osteoarthritis. Rheumatology (Oxford). 46(11): 1676-1683.

Fickert, S., J. Fiedler and R.E. Brenner. 2004. Identification of subpopulations with characteristics of mesenchymal progenitor cells from human osteoarthritic cartilage using triple staining for cell surface markers. Arthritis Res Ther. 6(5): R422-432. 


\section{Stem Cells and Bone Tissue}

Friedenstein, A.J., R.K. Chailakhjan and K.S. Lalykina. 1970. The development of fibroblast colonies in monolayer cultures of guinea-pig bone marrow and spleen cells. Cell Tissue Kinet. 3(4): 393-403.

Fuchs, E., T. Tumbar and G. Guasch. 2004. Socializing with the neighbors: stem cells and their niche. Cell. 116(6): 769-778.

Fuchs E. Finding one's nice in the skin. Cell Stem Cell 2009;4:499-502

Gronthos, S., A.C. Zannettino, S.J. Hay, S. Shi, S.E. Graves, A. Kortesidis and P.J. Simmons. 2003. Molecular and cellular characterisation of highly purified stromal stem cells derived from human bone marrow. J Cell Sci. 116(Pt 9): 1827-1835.

Haq, I., E. Murphy and J. Dacre. 2003. Osteoarthritis. Postgrad Med. J. 79: 377-383.

Johnstone, B., T.M. Hering, A.I. Caplan, V.M. Goldberg and J.U. Yoo. 1998. In vitro chondrogenesis of bone marrow-derived mesenchymal progenitor cells. Exp Cell Res. 238(1): 265-272.

Koelling, S. and N. Miosge. 2009. Stem cell therapy for cartilage regeneration in osteoarthritis. Expert Opinion on Biological Therapy. 9: 1-7.

Koelling, S., J. Kruegel, M. Irmer, J. Path, B. Sadowski, X. Miró and N. Miosge. 2009. Migratory chondrogenic progenitor cells from repair tissue during the late stages of human osteoarthritis. Cell Stem Cell. 4: 324-335.

Koelling S, Miosge N: Sex differences of chondrogenic progenitor cells in late stages of osteoarthritis. 2010. Arthritis Rheum 62:1077-1087.

Kouri, J.B., S.A. Jimenez, M. Quintero and A. Chico. 1996. Ultrastructural study of chondrocytes from fibrillated and non-fibrillated human osteoarthritic cartilage. Osteoarthritis Cartilage. 4: 111-125.

Kuettner, K.E. 1992. Biochemistry of articular cartilage in health and disease. Clin Biochem. 25: $155-163$.

Mackay, A.M., S.C. Beck, J.M. Murphy, F.P. Barry, C.O. Chichester and M.F. Pittenger. 1998. Chondrogenic differentiation of cultured human mesenchymal stem cells from marrow. Tissue Eng. 4(4): 415-428.

Martel-Pelletier, J. 1999. Pathophysiology of osteoarthritis. Osteoarthritis Cartilage. 6: 371-783.

Mayne, R. and R.G. Brewton. 1993. New members of the collagen superfamily. Curr Opin Cell Biol. 5: 883-890.

Miosge, N., K. Waletzko, C. Bode, F. Quondamatteo, W. Schultz and R. Herken. 1998. Light and electron microscopic in situ hybridization of collagen type I and type II mRNA in the fibrocartilaginous tissue of late-stage osteoarthritis. Osteoarthritis Cartilage. 6: 278-285.

Murphy, J.M., D.J. Fink, E.B. Hunziker and F.P. Barry. 2003. Stem cell therapy in a caprine model of osteoarthritis. Arthritis Rheum. 48(12): 3464-3474.

Murphy, L.M., K. Dixon, S. Beck, D. Fabian, A. Feldman and F. Barry. 2002. Reduced chondrogenic and adipogenic activity of mesenchymal stem cells from patients with advanced osteoarthritis. Arthritis Rheum. 46: 704-713.

O'Rahilly, R., J. Bossy and F. Muller. 1981. Introduction to the study of embryonic stages in man. Bull Assoc Anat (Nancy). 65(189): 141-236.

Poole A.R., Rizkalla G., Ionescu M., Reiner A., Brooks E., Rorabeck C., Bourne R. and BogochE. (1993). Osteoarthritis in the human knee: a dynamic process of cartilage matrix degradation, synthesis and reorganization. Agents Actions Suppl. 39, 3-13.

Poole, A.R. 1999. An introduction to the pathophysiology of osteoarthritis. Front Biosci. 4: D662-670.

Reginster, J.Y. 2002. The prevalence and burden of arthritis. Rheumatology 41 Supp1: 3-6.

Sandell, L.J. and T. Aigner. 2001. Articular cartilage and changes in arthritis. An Introduction: Cell biology of osteoarthritis. Arthritis Res. 3: 107-113.

Takahashi, K. and S. Yamanaka. 2006. Induction of pluripotent stem cells from mouse embryonic and adult fibroblast cultures by defined factors. Cell. 126(4): 663-676.

Wolbank, S., A. Peterbauer, M. Fahrner, S. Hennerbichler, M. van Griensven, G. Stadler, H. Redl and C. Gabriel. 2007. Dose-dependent immunomodulatory effect of human stem cells from amniotic membrane: a comparison with human mesenchymal stem cells from adipose tissue. Tissue Eng. 13(6): 1173-1183.

Woolf, A.D. and B. Pfleger. 2003. Burden of major musculoskeletal conditions. Bull. World Health Organ. 81: 646-656. 


\section{Conclusion}

$\mathrm{OA}$ is a disease of cartilage degeneration, in worse conditions, as in the later stages of $\mathrm{OA}$, the uncovered bone ends rub against each other and lead to the deformation or even complete loss of the joint. Total joint replacement is considered as an 'ultimata ratio' in the later stages of OA. However, as a cellular therapy, stem cells are considered as a good choice for cartilage tissue regeneration. Numerous types of stem cells have been introduced for cartilage regeneration, for example, ESCs derived from the inner cells mass of the embryo, or cells from the adipose tissue, the bone marrow and synovial membrane. These cells sources revealed somewhat encouraging results, however, they also have disadvantages. Prof. Miosge's lab discovered a tissue specific source of cells already present in the diseased cartilage, termed 'chondrogenic progenitor cells' (CPCs). The CPCs have stem cells-like characteristics and have the ability to differentiate into chondrocytes. CPCs isolated from the late stages of human osteoarthritic cartilage are determined to the osteo-chondrogenic lineage. However, they could be easily driven towards chondrogenesis (chapter 1).

DDR-1 KO mice showed typical symptoms of OA of the TMJ, as early as 9-weeks of age. The TMJ cartilage exhibited osteoarthritic features, such as surface fissures, low proteoglycan content, and cell clusters. Collagen type I was highly up-regulated and was structurally disorganized, as confirmed by transmission electron microscopy. Furthermore, DDR-2, which is considered a gene associated with OA, was up-regulated. The cells isolated from TMJ cartilage of the DDR-1 KO mice maintained their typical osteoarthritic characteristics in vitro as well. For examples, as compared to wild type, the chondrocytes derived from TMJ cartilage of the DDR-1 KO mice showed high expressions of collagen type I, DDR-2, and MMP-13. However these cells showed low expression of sox 9 and collagen type II, the major transcription factor and the main ECM protein of healthy hyaline cartilage, respectively. All the above findings have been confirmed with FACS analyses and microarray data. Additionally, mouse signal transduction PCR array experiments revealed the regulation of other important candidate genes, associated with OA or DDR-1-linked downstream players, particularly the involvement of the $\mathrm{HH}$ (indian hedgehog) pathway. Two strategies were applied in order to reverse the osteoarthritic signs of these cells towards normal chondrocytes. First, the runx2 knockdown resulted in the complete loss of collagen type I, up-regulation of sox 9 
and collagen type II. Secondly, the cells were cultured in a three-dimensional environment in the presence of nidogen-2, BMP-6 or laminin-1. This second approach also revealed positive influences on the cells driving them towards chondrogenesis. For example, nidogen-2 and laminin-1 significantly reduced runx2 and collagen type I, respectively. Furthermore, a reduction was found in the intensity of the staining for primary cilia on DDR-1 KO cells, which indicates a connection of the primary cilium with OA of the TMJ. No osteoarthritic symptoms were observed in the larger joints of DDR-1 $\mathrm{KO}$ mice, which clearly depicts that the DDR-1 function is restricted to the homeostasis of TMJ cartilage. Moreover, another possible explanation would be that embryonically the TMJ cartilage and knee cartilage originate from different progenitors cell pools.

Numerous studies showed the involvement of the primary cilium in stem cell differentiation. The primary cilium has been shown on CPCs via immunocytochemical techniques using antibodies against acetylated alpha-tubulin. Surprisingly, the intensity of primary cilium staining was reduced in chondrocytes derived from the TMJ cartilage of DDR-1 KO mouse. This indicates that the primary cilium is not only involved in cell differentiation but also in the pathogenesis of cartilage (chapter 2 and chapter 3 ).

Meniscus degradation is considered as one of the major causes of OA. Over all, more than 150 surgical specimens of human late stage osteoarthritic menisci were examined for this study. The degradation process was noticed predominantly in the superficial zone of the meniscus. Therefore, the inner zone of meniscus was taken for tissue explant cultures. Interestingly, the cells were migrating out only from those menisci lacking a superficial zone. However, the cells from the superficial zone and inner zone of the healthy meniscus, with an intact superficial zone, could be obtained via collagenase digestion. They maintained their different phenotypes also in vitro. The inner zone cells showed a round phenotype. While the superficial zone cells were found to exhibit a spindle shaped phenotype. Total proteome and transcriptome data analyses brought many others genes to light typically associated with $O A$. These genes were found highly regulated, such as, TIMP2, sox9, ACAN and MMP14. Over all, a severe catabolic shift was noticed in diseased meniscus.

FACS analyses exhibited highly positive signal intensities for stem cell markers (CD105, CD90, CD44, CD29, stro-1) in inner zone cells derived via explant cell culture as 
compared to cells derived via digestion from the inner zone of less diseased menisci. They were found to be also multipotent and could be differentiated into the three mesenchymal lineages (adipocytes, chondrocytes and cells of the osteoblastic lineage). Furthermore, these cells showed a high migratory activity towards a chemotactant, the human recombinant platelet derived growth factor (PDGF). Taken together, these distinctive characteristics allowed to name them MPCs. Furthermore, cell integration experiments were performed to check their possible behavior ex-vivo. The GFP-labeled MPCs showed a high penetration and integration capability into diseased meniscus tissue. The incorporation of the MPCs was traced via immunofluorescence and immunohistochemical techniques using antibodies against GFP.

Microarray and proteome data of diseased cells also revealed the suppression of the transforming growth factor beta (TGF $\beta$ ) pathway, a signal transduction pathways which favors chondrogenesis. In vivo and in vitro, numerous experiments showed that TGF $\beta 3$ protein (extracellular ligand of the TGF $\beta$ pathway), the cytoplasmic proteins smad2/smad3 (mediators) and the nucleoplasmic protein sox9 (target gene, as downstream transcription factor) were significantly reduced in highly diseased human meniscus tissue and in cells in vitro. Importantly, the inhibitor of these pathways, e.g., smad7 was highly elevated in diseased samples. Besides this, the expression of the osteogenic master regulator 'runx2' was elevated in diseased samples, which suppressed the smad2 expression. Molecular and cell biological techniques were applied in order to guide MPCs towards the chondrogenic lineage. For examples, the gene silencing experiment of runx2, switched on the TGF $\beta$ pathway via the active mediator phosphorylated smad2 (p-smad2). Here, at this point, I hypothesized that both proteins, runx2 and smad2, are acting like antagonist and agonist, respectively. In a cell induction experiment with TGF $\beta 3$, the ligand triggered the signal towards the cell cytoplasm via its membrane receptors (type II and type I receptor kinase). The membrane receptor activates the cytoplasmic mediators (smad2/3) via phosphorylation. Further, the mediator (smad2/3) cross-talks with smad4, and finally this complex translocates to the nucleus and starts the transcription of the downstream target gene (sox9). All these steps were followed at the transcriptional level (via rt-PCR) and at the translational level (via Western blots). Interestingly, active smad2 (p-smad) enhanced the sox9 expression and the antagonist (runx2) was highly suppressed. Further, the opposing effect of runx2 was 
confirmed by a cell induction experiment with BMP: runx2 expression was elevated and sox9 was down-regulated. As further evidence of this antagonism, overexpression experiments were performed. The smad2 overexpression significantly reduced runx 2 . Taken together, these observations suggest that MPCs can be controlled via the TGF $\beta$ pathway for the regeneration of the human meniscus, this might lead to new therapeutic options (chapter 4).

Mouse meniscus also harbors progenitor cells, which are positive for well-known stem cells markers such as CD29, CD73 and CD105. MMPCs cells were localized in vivo via immunohistochemical techniques. Interestingly, the round cells of the inner zone were found positive for stem cells markers, as already seen in humans. Stem cell-related cell surface antigens were confirmed at the transcriptional level, via rt-PCR, and the translational level, applying Western blot and FACS analyses. These cells showed multipotency and could be differentiated only into two mesenchymal lineages. Histologically, at the cellular level the mouse meniscus revealed different features than the human meniscus. For example, the mouse meniscus lacks the superficial zone. Moreover, other distinguishable features were observed by immunohistochemistry, for example, collagen type I staining was found throughout the inner zones of the inner and outer parts of the human meniscus, while collagen type I staining was restricted to outer zone of the mouse meniscus (chapter 5).

In summary, OA cartilage degeneration overrides the generally limited self-renewal capacity of the tissue. It remains a great challenge to enhance the regeneration potential of hyaline cartilage and human meniscus during $O A$. In the long run the intrinsic efforts of repair cells lead to scar tissue, which is not flexible and biomechanically unfeasible for proper joint moments. Here, progenitor cells in diseased meniscus in situ were introduced, which are a starting point for cell biological therapeutic options for cartilage repair in the future (chapter 6) 


\section{References}

Arnoczky SP, Warren RF (1983) The microvasculature of the meniscus and its response to injury. An experimental study in the dog. Am J Sports Med 11: 131-141

Banerjee C, McCabe LR, Choi JY, Hiebert SW, Stein JL, Stein GS, Lian JB (1997) Runt homology domain proteins in osteoblast differentiation: AML3/CBFA1 is a major component of a bone-specific complex. Journal of cellular biochemistry 66: 1-8

Bi W, Deng JM, Zhang Z, Behringer RR, de Crombrugghe B (1999) Sox9 is required for cartilage formation. Nature genetics 22: 85-89

Bian L, Zhai DY, Mauck RL, Burdick JA (2011) Coculture of human mesenchymal stem cells and articular chondrocytes reduces hypertrophy and enhances functional properties of engineered cartilage. Tissue Eng Part A 17: 1137-1145

Blagojevic M, Jinks C, Jeffery A, Jordan KP (2010) Risk factors for onset of osteoarthritis of the knee in older adults: a systematic review and meta-analysis. Osteoarthritis Cartilage 18: 24-33

Chevrier A, Nelea M, Hurtig MB, Hoemann CD, Buschmann MD (2009) Meniscus structure in human, sheep, and rabbit for animal models of meniscus repair. Journal of orthopaedic research : official publication of the Orthopaedic Research Society 27: 1197-1203

Cui X, Hasegawa A, Lotz M, D'Lima D (2012) Structured three-dimensional co-culture of mesenchymal stem cells with meniscus cells promotes meniscal phenotype without hypertrophy. Biotechnol Bioeng 109: 2369-2380

Eyre DR, Wu JJ (1983) Collagen of fibrocartilage: a distinctive molecular phenotype in bovine meniscus. FEBS letters 158: 265-270

Ferris D, Frisbie D, Kisiday J, Mcllwraith CW (2012) In vivo healing of meniscal lacerations using bone marrow-derived mesenchymal stem cells and fibrin glue. Stem Cells Int 2012: 691605

Foehr ED, Tatavos A, Tanabe E, Raffioni S, Goetz S, Dimarco E, De Luca M, Bradshaw RA (2000) Discoidin domain receptor 1 (DDR1) signaling in PC12 cells: activation of juxtamembrane domains in PDGFR/DDR/TrkA chimeric receptors. FASEB journal : official publication of the Federation of American Societies for Experimental Biology 14: 973981

Goldring MB (2012) Chondrogenesis, chondrocyte differentiation, and articular cartilage metabolism in health and osteoarthritis. Therapeutic advances in musculoskeletal disease 4: $269-285$

Goldring MB, Otero M (2011) Inflammation in osteoarthritis. Current opinion in rheumatology 23: 471-478 
Gouttenoire J, Valcourt U, Bougault C, Aubert-Foucher E, Arnaud E, Giraud L, MalleinGerin F (2007) Knockdown of the intraflagellar transport protein IFT46 stimulates selective gene expression in mouse chondrocytes and affects early development in zebrafish. The Journal of biological chemistry 282: 30960-30973

Hasan J, Fisher J, Ingham E (2014) Current strategies in meniscal regeneration. Journal of biomedical materials research Part B, Applied biomaterials 102: 619-634

Haycraft CJ, Zhang Q, Song B, Jackson WS, Detloff PJ, Serra R, Yoder BK (2007) Intraflagellar transport is essential for endochondral bone formation. Development 134: 307-316

Heatley FW (1980) The meniscus--can it be repaired? An experimental investigation in rabbits. The Journal of bone and joint surgery British volume 62: 397-402

Hellio Le Graverand MP, Ou Y, Schield-Yee T, Barclay L, Hart D, Natsume T, Rattner JB (2001a) The cells of the rabbit meniscus: their arrangement, interrelationship, morphological variations and cytoarchitecture. Journal of anatomy 198: 525-535

Hellio Le Graverand MP, Vignon E, Otterness IG, Hart DA (2001b) Early changes in lapine menisci during osteoarthritis development: Part II: molecular alterations. Osteoarthritis and cartilage / OARS, Osteoarthritis Research Society 9: 65-72

Henning CE, Lynch MA, Yearout KM, Vequist SW, Stallbaumer RJ, Decker KA (1990) Arthroscopic meniscal repair using an exogenous fibrin clot. Clinical orthopaedics and related research: $64-72$

Herwig J, Egner E, Buddecke E (1984) Chemical changes of human knee joint menisci in various stages of degeneration. Annals of the rheumatic diseases 43: 635-640

Ingman AM, Ghosh P, Taylor TK (1974) Variation of collagenous and non-collagenous proteins of human knee joint menisci with age and degeneration. Gerontologia 20: 212223

Ito MM, Kida MY (2000) Morphological and biochemical re-evaluation of the process of cavitation in the rat knee joint: cellular and cell strata alterations in the interzone. Journal of anatomy $197 \mathrm{Pt}$ 4: 659-679

Jensen CG, Poole CA, McGlashan SR, Marko M, Issa ZI, Vujcich KV, Bowser SS (2004) Ultrastructural, tomographic and confocal imaging of the chondrocyte primary cilium in situ. Cell biology international 28: 101-110

Kamekura S, Kawasaki Y, Hoshi K, Shimoaka T, Chikuda H, Maruyama Z, Komori T, Sato S, Takeda S, Karsenty G, Nakamura K, Chung UI, Kawaguchi H (2006) Contribution of runt-related transcription factor 2 to the pathogenesis of osteoarthritis in mice after induction of knee joint instability. Arthritis and rheumatism 54: 2462-2470

Katagiri H, Muneta T, Tsuji K, Horie M, Koga H, Ozeki N, Kobayashi E, Sekiya I (2013) Transplantation of aggregates of synovial mesenchymal stem cells regenerates meniscus 
more effectively in a rat massive meniscal defect. Biochem Biophys Res Commun 435: 603-609

King D (1990) The healing of semilunar cartilages. 1936. Clinical orthopaedics and related research: 4-7

Knudson CB, Knudson W (2001) Cartilage proteoglycans. Seminars in cell \& developmental biology 12: 69-78

Koelling S, Kruegel J, Irmer M, Path JR, Sadowski B, Miro X, Miosge N (2009) Migratory chondrogenic progenitor cells from repair tissue during the later stages of human osteoarthritis. Cell Stem Cell 4: 324-335

Koelling S, Miosge N (2010) Sex differences of chondrogenic progenitor cells in late stages of osteoarthritis. Arthritis and rheumatism 62: 1077-1087

Kohn D, Moreno B (1995) Meniscus insertion anatomy as a basis for meniscus replacement: a morphological cadaveric study. Arthroscopy : the journal of arthroscopic \& related surgery : official publication of the Arthroscopy Association of North America and the International Arthroscopy Association 11: 96-103

Kruegel J, Sadowski B, Miosge N (2008) Nidogen-1 and nidogen-2 in healthy human cartilage and in late-stage osteoarthritis cartilage. Arthritis and rheumatism 58: 14221432

Kuettner KE (1992) Biochemistry of articular cartilage in health and disease. Clinical biochemistry 25: 155-163

Lefebvre V, Smits P (2005) Transcriptional control of chondrocyte fate and differentiation. Birth defects research Part C, Embryo today : reviews 75: 200-212

Lin AC, Seeto BL, Bartoszko JM, Khoury MA, Whetstone H, Ho L, Hsu C, Ali SA, Alman BA (2009) Modulating hedgehog signaling can attenuate the severity of osteoarthritis. Nature medicine 15: 1421-1425

Loeser RF (2000) Chondrocyte integrin expression and function. Biorheology 37: 109116

Loeser RF, Goldring SR, Scanzello CR, Goldring MB (2012) Osteoarthritis: a disease of the joint as an organ. Arthritis and rheumatism 64: 1697-1707

Massague J (2012) TGFbeta signalling in context. Nature reviews Molecular cell biology 13: $616-630$

Mauck RL, Yuan X, Tuan RS (2006) Chondrogenic differentiation and functional maturation of bovine mesenchymal stem cells in long-term agarose culture. Osteoarthritis Cartilage 14: 179-189 
Mayne R, Brewton RG (1993) New members of the collagen superfamily. Current opinion in cell biology 5: 883-890

McDevitt CA, Webber RJ (1990) The ultrastructure and biochemistry of meniscal cartilage. Clinical orthopaedics and related research: 8-18

McGlashan SR, Cluett EC, Jensen CG, Poole CA (2008) Primary cilia in osteoarthritic chondrocytes: from chondrons to clusters. Developmental dynamics : an official publication of the American Association of Anatomists 237: 2013-2020

McGlashan SR, Jensen CG, Poole CA (2006) Localization of extracellular matrix receptors on the chondrocyte primary cilium. The journal of histochemistry and cytochemistry : official journal of the Histochemistry Society 54: 1005-1014

Mikic B, Johnson TL, Chhabra AB, Schalet BJ, Wong M, Hunziker EB (2000) Differential effects of embryonic immobilization on the development of fibrocartilaginous skeletal elements. Journal of rehabilitation research and development 37: 127-133

Muhammad H, Rais Y, Miosge N, Ornan EM (2012) The primary cilium as a dual sensor of mechanochemical signals in chondrocytes. Cellular and molecular life sciences : CMLS 69: 2101-2107

Muhammad H, Schminke B, Miosge N (2013) Current concepts in stem cell therapy for articular cartilage repair. Expert Opin Biol Ther 13: 541-548

Noble J, Hamblen DL (1975) The pathology of the degenerate meniscus lesion. J Bone Joint Surg Br 57: 180-186

Osawa A, Harner CD, Gharaibeh B, Matsumoto T, Mifune Y, Kopf S, Ingham SJ, Schreiber V, Usas A, Huard J (2013) The use of blood vessel-derived stem cells for meniscal regeneration and repair. Med Sci Sports Exerc 45: 813-823

Pavlova A, Gamer L, Cox K, Celeste A, Rosen V (2001) Developmental expression of BMPs and matrix proteins during meniscal pathogenesis. Transactions, vol. 26, San Francisco, California

Pauli C, Grogan SP, Patil S, Otsuki S, Hasegawa A, Koziol J, Lotz MK, D'Lima DD (2011) Macroscopic and histopathologic analysis of human knee menisci in aging and osteoarthritis. Osteoarthritis Cartilage 19: 1132-1141

Pelttari K, Winter A, Steck E, Goetzke K, Hennig T, Ochs BG, Aigner T, Richter W (2006) Premature induction of hypertrophy during in vitro chondrogenesis of human mesenchymal stem cells correlates with calcification and vascular invasion after ectopic transplantation in SCID mice. Arthritis Rheum 54: 3254-3266

Petersen W, Tillmann B (1995) Age-related blood and lymph supply of the knee menisci. A cadaver study. Acta orthopaedica Scandinavica 66: 308-312 
Petersen W, Tillmann B (1998) Collagenous fibril texture of the human knee joint menisci. Anatomy and embryology 197: 317-324

Poole AR (1999) An introduction to the pathophysiology of osteoarthritis. Frontiers in bioscience : a journal and virtual library 4: D662-670

Poole CA, Jensen CG, Snyder JA, Gray CG, Hermanutz VL, Wheatley DN (1997) Confocal analysis of primary cilia structure and colocalization with the Golgi apparatus in chondrocytes and aortic smooth muscle cells. Cell biology international 21: 483-494

Poole CA, Zhang ZJ, Ross JM (2001) The differential distribution of acetylated and detyrosinated alpha-tubulin in the microtubular cytoskeleton and primary cilia of hyaline cartilage chondrocytes. Journal of anatomy 199: 393-405

Rintala M, Metsaranta M, Saamanen AM, Vuorio E, Ronning O (1997) Abnormal craniofacial growth and early mandibular osteoarthritis in mice harbouring a mutant type II collagen transgene. Journal of anatomy 190 ( Pt 2): 201-208

Ruiz-Perez VL, Blair HJ, Rodriguez-Andres ME, Blanco MJ, Wilson A, Liu YN, Miles C, Peters H, Goodship JA (2007) Evc is a positive mediator of Ihh-regulated bone growth that localises at the base of chondrocyte cilia. Development 134: 2903-2912

Sakaguchi Y, Sekiya I, Yagishita K, Muneta T (2005) Comparison of human stem cells derived from various mesenchymal tissues: superiority of synovium as a cell source. Arthritis Rheum 52: 2521-2529

Schminke B, Muhammad H, Bode C, Sadowski B, Gerter R, Gersdorff N, Burgers R, Monsonego-Ornan E, Rosen V, Miosge N (2014) A discoidin domain receptor 1 knockout mouse as a novel model for osteoarthritis of the temporomandibular joint. Cellular and molecular life sciences : CMLS 71: 1081-1096

Shen G, Darendeliler MA (2005) The adaptive remodeling of condylar cartilage---a transition from chondrogenesis to osteogenesis. Journal of dental research 84: 691-699

Shen J, Li J, Wang B, Jin H, Wang M, Zhang Y, Yang Y, Im HJ, O'Keefe R, Chen D (2013) Deletion of the transforming growth factor beta receptor type II gene in articular chondrocytes leads to a progressive osteoarthritis-like phenotype in mice. Arthritis and rheumatism 65: 3107-3119

Silbermann M, Livne E (1979) Age-related degenerative changes in the mouse mandibular joint. Journal of anatomy 129: 507-520

Symons NB (1965) A histochemical study of the secondary cartilage of the mandibular condyle in the rat. Archives of oral biology 10: 579-584

Tallheden T, Karlsson C, Brunner A, Van Der Lee J, Hagg R, Tommasini R, Lindahl A (2004) Gene expression during redifferentiation of human articular chondrocytes. Osteoarthritis and cartilage / OARS, Osteoarthritis Research Society 12: 525-535 
Vogel W (1999) Discoidin domain receptors: structural relations and functional implications. FASEB journal : official publication of the Federation of American Societies for Experimental Biology 13 Suppl: S77-82

Vogel W, Gish GD, Alves F, Pawson T (1997) The discoidin domain receptor tyrosine kinases are activated by collagen. Molecular cell 1: 13-23

Wadhwa S, Embree M, Ameye L, Young MF (2005) Mice deficient in biglycan and fibromodulin as a model for temporomandibular joint osteoarthritis. Cells, tissues, organs 181: $136-143$

Walker PS, Erkman MJ (1975) The role of the menisci in force transmission across the knee. Clin Orthop Relat Res: 184-192

Wilsman NJ (1978) Cilia of adult canine articular chondrocytes. Journal of ultrastructure research 64: 270-281

Wilsman NJ, Farnum CE, Reed-Aksamit DK (1980) Incidence and morphology of equine and murine chondrocytic cilia. The Anatomical record 197: 355-361

Wuelling M, Vortkamp A (2011) Chondrocyte proliferation and differentiation. Endocrine development 21: 1-11

Xu L, Flahiff CM, Waldman BA, Wu D, Olsen BR, Setton LA, Li Y (2003) Osteoarthritis-like changes and decreased mechanical function of articular cartilage in the joints of mice with the chondrodysplasia gene (cho). Arthritis and rheumatism 48: 2509-2518

Xu L, Peng H, Glasson S, Lee PL, Hu K, ljiri K, Olsen BR, Goldring MB, Li Y (2007) Increased expression of the collagen receptor discoidin domain receptor 2 in articular cartilage as a key event in the pathogenesis of osteoarthritis. Arthritis and rheumatism 56: 2663-2673

Zhang D, Cheriyan T, Martin SD, Gomoll AH, Schmid TM, Spector M (2011) Lubricin distribution in the torn human anterior cruciate ligament and meniscus. J Orthop Res 29: 1916-1922

Zhang Z, Arnold JA, Williams T, McCann B (1995) Repairs by trephination and suturing of longitudinal injuries in the avascular area of the meniscus in goats. The American journal of sports medicine 23: 35-41 


\section{Acknowledgments}

I would like to express my deepest gratitude to Prof. Dr. Nicolai Miosge for offering the opportunity to do Ph.D. research in his Tissue Regeneration Work Group. It was a pleasure and an honor to work under his supervision and in the exciting scientific environment of the tissue regeneration laboratory.

I would like to express my profound gratitude to my second supervisor Prof. Dr. Sigrid Hoyer-Fender for her tremendous scientific suggestions, constructive critics and fruitful discussion throughout my Ph.D. research.

My sincere gratitude to all of my Ph.D. board exam members (Prof. Dr. Gehard Braus Prof. Dr. Uwe Gross, Prof. Dr. Micheal Kessel, Prof. Dr. Ernst Wimmer) for reviewing my Ph.D. research work.

I am highly grateful and thankful to my research collaborative Prof. Dr. Vicki Rosen from Harvard University, USA, and Prof. Dr. Efrat Monsonego Ornan from Hebrew University, Israel and Prof. Dr. Mary Goldring from Cornell University, USA, for their valuable discussion and excellent guidance.

I offer special thanks to Dr. Boris Schminke, for his consistent scientific guidance, motivation, and inspiring discussion. He is a brilliant mentor and always friendly to all colleagues.

I sincerely thank all of the laboratory technicians, especially Elke Henze-Santogeanis, Christa Bode, Boguslawa Sadowski, and Andrea Bernhard for their technical support, expertise and always available kind help.

I was blessed to work in such a fantastic, inspiring and friendly atmosphere of nice and lovely colleagues Andrea, Jenny, Vanessa, Angelika, Regina, Sarah, Airin, Mirjana, Antonia, Moritz, Julius, Gökhan, Martin, Simon, Robert, Gunar, Stefen, Phillip, Tolga, Marcus, and Jan. I never felt tired of lab work with such amazing research group members. I enjoyed with them the weekly journal club, scientific discussions at daily lunchtime and outside fun in the city.

My special thanks to my colleague and friend Matz, who taught me the German grammar and I enjoyed badminton sport with him.

Many thanks to my friends Dr. Adnan, Dr. Malik, Dr. Hazir, Dr. Qasim, Dr. Murad, Naeem Khan, Asif Khan and Junaid Khan for their motivation and scientific discussion.

My special thanks to my uncle Sher Afzal Khan, my brother engineer Nisar Muhammad, little sister Dolly and little cousin Gori for their kind support and prayers throughout my academic career. 
At last but not least, I would like to express my deepest gratitude to my parents for their moral support, encouragement and prayers throughout my academic career.

Hayat Muhammad 


\section{Curriculum Vitae: Hayat Muhammad}

Date and Place of Birth: $8^{\text {th }}$ April, 1980, Darazinda F.R. Dera Ismail Khan, KPK, Pakistan.

\section{EDUCATION:}

$2010-2014$

2001-2004

1998-2000

1996-1998

1994-1996

\section{RESEARCH ACTIVITIES}

2010-2014

Ph.D. Research student, at Tissue Regeneration Research Group, Department of Prosthodontics, Georg August University, Goettingen, Germany

$2009-2010$

Max-Planck Research Fellow, an internship at Department for NMR based Structural Biology, Max-Planck Institute for Biophysical Chemistry, Goettingen, Germany.

2005-2008
Junior Research Fellow as structural biologist, at International for Chemical and Biological Sciences, University of Karachi, Karchi, 75270, Pakistan. 


\section{PUBLICATIONS}

Hayat Muhammad, Yoach Rais, Nicolai Miosge and Efrat Monsonego Ornan "Primary cilium as dual sensor of mechanochemical signals in chondrocytes" Cell Mol Life Sci. 2012 Jul; 69 (13): $2101-7$.

Hayat Muhammad, Boris Schminke and Nicolai Miosge "Current concepts in stem cell therapy for articular cartilage repair" Expert Opin Biol Ther. April 2013, Vol. 13, No. 4, Pages 541-548.

Boris Schminke*, Hayat Muhammad*, Christa Bode, Boguslawa Sadowski, Regina Gerter, Nikolaus Gersdorff, Ralf Bürgers, Efrat Monsonego-Ornan, Vicki Rosen and Nicolai Miosge "Discoidin domain receptor 1 knock-out mice as a novel model for osteoarthritis of the temporomandibular joint" Cell Mol Life Sci. 2013 Aug 4 [Epub ahead of print]

*Both authors contributed equally.

Hayat Muhammad*, Boris Schminke*, Christa Bode, Andrea Bernhard, Moritz Roth, Julius Albert, Silvia von der Heyde, Vicki Rosen and Nicolai Miosge "Human migratory meniscus progenitor cells are controlled via the TGFB pathway. (Submitted)

*Both authors contributed equally.

Hayat Muhammad, Matz Menrath, Boris Schminke, Vicki Rosen and Nicolai Miosge "Identification and characterization of mouse meniscal progenitor stem cells in vitro and ex vivo" (manuscript under under submission).

\section{BOOK CHAPTER}

Boris Schminke, Nicolai Miosge and Hayat Muhammad "Use of chondrogenic progenitor cells in osteoarthritis" Chapter No. 13, Stem cells and Bone Tissue. January 23, 2013, CRC Press. 